\title{
Experimentelle Untersuchungen zu Mechanismen von druck- und temperaturinduzierten Phasenübergängen
}

\author{
Dissertation \\ zur Erlangung des
}

mathematisch-naturwissenschaftlichen Doktorgrades

"Doctor rerum naturalium"

der Georg-August-Universität Göttingen

im Promotionsprogramm Geoscience
der Georg-August University School of Science (GAUSS)

vorgelegt von

Nina Katrin Pukallus

aus Bremen

Göttingen, den 09. 09. 2020 
Ich möchte hiermit folgenden Personen meinen Dank für die Unterstützung bzw. Betreuung sowohl im Vorfeld als auch während der Erstellung dieser Arbeit aussprechen:

- Dr. Heidrun Sowa für die ausführliche Einführung in die Welt der Hochdruckmessungen, die sehr gute Betreuung und die Möglichkeit, die Dissertation doch noch zu einem Ende zu führen

- Dr. Helmut Klein für das Teilen seines unerschöpflichen Wissens über das Thema Texturen, die sehr gute Betreuung und die Möglichkeit, die Dissertation doch noch zu einem Ende zu führen

- Dr. Lars Raue für die Hilfe bei der Versuchsdurchführung und die Bürogemeinschaft

- Imke Janßen für ihre Freundschaft, die Bürogemeinschaft und die gegenseitige Motivation

- Ulf Kahmann für die technische Unterstützung am GZG

- Prof. Dr. Sharon Webb für die Übernahme des Platzes von Prof. Dr. Werner F. Kuhs im Betreuungsausschuss nach dessen Renteneintritt

- Den Mitarbeiter*innen des HASYLAB, Hamburg, für die technische Unterstützung während der Messzeiten

- Der Deutschen Forschungsgemeinschaft für die anfängliche Finanzierung des Projekts (Projektnummer SO 276/3-1).

- Der Arbeitsgemeinschaft Texturen e.V. für die Gewährung eines Stipendiums zur Fortführung des Projekts nach Ende der Finanzierung durch die DFG.

- Bayek von Siwa, dessen Abenteuer mich in den letzten Monaten der Dissertation begleitet und meinen Drang nach Eskapismus angesichts der Coronakrise gestillt haben. 


\section{Inhaltsverzeichnis}

1 Einleitung $\quad 5$

2 Theoretische Grundlagen $\quad 7$

2.1 Kristalline Materialien . . . . . . . . . . . . . . . . 7

2.2 Orientierung . . . . . . . . . . . . . . . . . . . . . . . 7

2.2 .1 Eulerwinkel . . . . . . . . . . . . . . . . . . 8

2.2.2 Matrixdarstellung . . . . . . . . . . . . . . . . 9 9

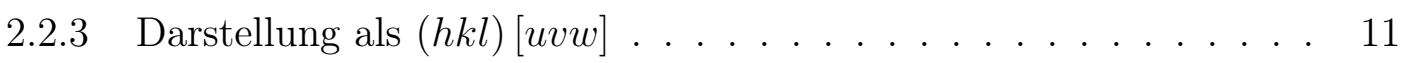

2.3 Der Texturbegriff . . . . . . . . . . . . . . . . . . . 13

2.3.1 Die Bragg-Bedingung . . . . . . . . . . . . . . . . . 14

2.4 Darstellung von Polfiguren . . . . . . . . . . . . . . . . 16

2.4.1 Definition einer Polfigur . . . . . . . . . . . . . 16

2.4.2 Das Wulffsche Netz . . . . . . . . . . . . . . . . . . . . . 16

2.5 Berechnung der ODF aus Polfiguren . . . . . . . . . . . . . 18

2.5.1 Die Fundamentalgleichung der Texturanalyse . . . . . . . . . . . 18

2.5.2 Die Harmonische Methode . . . . . . . . . . . . . . . . . . . . . . 19

2.5.3 Die E-WIMV-Methode . . . . . . . . . . . . . . . . . . 24

2.6 Phasenumwandlungen . . . . . . . . . . . . . . . . 26

2.7 Unter- und Obergruppen kristallographischer Raumgruppen und Transformationspfade . . . . . . . . . . . . . . . . . . . . 30

2.7.1 Unter- und Obergruppen . . . . . . . . . . . . . . . . 30

2.7.2 Transformationspfade................ . 31

2.7.3 Achsen- und Koordinatentransformationen . . . . . . . . . . . . 36

3 Die Probensubstanzen und ihre kristallographischen Daten 39

3.1 Indiumarsenid $(\operatorname{InAs}) \quad \ldots \ldots \ldots \ldots$. . . . . . . . . . . . . . . . . . . . . .

3.2 Cadmiumsulfid $(C d S) \quad \ldots \ldots \ldots \ldots$. . . . . . . . . . . 40 
3.3 Cadmiumselenid $(C d S e) \ldots \ldots \ldots \ldots$. . . . . . . . . . . . . 41

3.4 Kupferindiumdiselenid $\left(\mathrm{CuInSe}_{2}\right) \ldots \ldots \ldots \ldots$. . . . . . . . 41

3.5 Nickelsulfid $(N i S) \quad \ldots \ldots \ldots \ldots \ldots$

4 Probenpräparation $\quad 44$

4.1 Hochdruckexperimente . . . . . . . . . . . . . . . . . 44

4.2 Hochtemperaturexperimente . . . . . . . . . . . . . 47

5 Versuchsdurchführung $\quad 49$

5.1 Erzeugung von Synchrotronstrahlung . . . . . . . . . . . . . 49

5.2 Beamlines BW5 und W2, DORIS III . . . . . . . . . . . . . . . 51

5.3 Beamline P02.1, PETRA III . . . . . . . . . . . . . . . . . . 53

5.4 Hochdruckexperimente . . . . . . . . . . . . . . . 53

5.5 Hochtemperaturexperimente . . . . . . . . . . . . . . . . . 54

6 Auswertung $\quad 56$

6.1 Vom Detektorbild zur Polfigur . . . . . . . . . . . . . 56

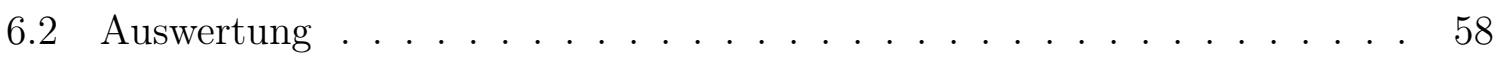

7 Ergebnisse und Interpretation $\quad 64$

7.1 Indiumarsenid $($ InAs $) \ldots \ldots \ldots$. . . . . . . . . . . . 64

7.2 Cadmiumsulfid $(C d S)$ und Cadmiumselenid $(C d S e) \ldots \ldots . \ldots 78$

7.3 Kupferindiumdiselenid $\left(\mathrm{CuInSe}_{2}\right) \ldots \ldots \ldots \ldots$. . . . . . . . 90

7.4 Nickelsulfid $(N i S) \quad \ldots \ldots \ldots \ldots$. . . . . . . . . . . . . 96

8 Zusammenfassung und Ausblick 103

9 Literaturverzeichnis $\quad 105$

$\begin{array}{ll}10 \text { Anhang } & 114\end{array}$ 


\section{Einleitung}

Viele kristalline Materialien treten abhängig von den Druck- und Temperaturbedingungen in ihrer Umgebung mit unterschiedlichen Kristallstrukturen auf. Die Kristallstruktur sowie die Ausrichtung des Kristallgitters haben großen Einfluss auf die physikalischen Eigenschaften der Materialien. Deshalb ist das Wissen über die Neuausrichtung des Kristallgitters nach einer Phasenumwandlung zum Beispiel bei Werkstoffen, die unter schwankenden Umweltbedingungen eingesetzt werden von großem Interesse. So sind Materialien zum Beispiel in der Raumfahrttechnik oder in Hochtemperatur- und Hochdruckanwendungen in der Industrie extremen Umweltbedingungen ausgesetzt und müssen sich auch unter diesen bewähren. Ebenso spielt eine neue Orientierung der Kristallite eines Gesteins bei einer Phasenumwandlung im Zuge gesteinsbildender Prozesse wie metamorpher Überprägung eine Rolle bei der Änderung der physikalischen Eigenschaften des Gesteins und kann somit eine Auswirkung auf tektonische Prozesse haben.

Zur Bestimmung der Orientierungsbeziehungen verschiedener Phasen eines Materials nach einer Phasenumwandlung 1. Art existieren bereits Veröffentlichungen von H. Sowa ([72], [73]) zu den Materialien $C d S$ und $C d S e$, welche auf Aufnahmen auf Röntgenfilm basieren, sowie eine Veröffentlichung von Sowa, Klein und Raue ([77]), welche sich mit den Orientierungsbeziehungen zwischen Tieftemperatur- und Hochtemperaturphase von Millerit beschäftigt. Das Ziel der vorliegenden Arbeit ist sowohl der experimentelle Beweis, dass bei Materialien, die eine Phasenumwandlung 1. Art durchlaufen, Orientierungsbeziehungen zwischen den Phasen existieren, als auch die Wiederholung der bereits durchgeführten Experimente mit moderneren Mess- und Auswertungsmethoden um die bereits gewonnenen Ergebnisse zu bestätigen oder zu widerlegen. Hierzu gehört, wenn möglich, die Bestimmung der Art der Orientierungsbeziehungen zwischen den verschiedenen Phasen sowie den Transformationspfad über eine gemeinsame Untergruppe der Raumgruppen beiden Phasen.

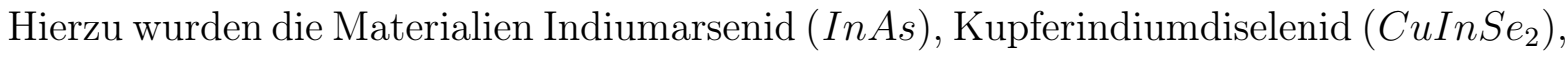


sowie für die Wiederholungsmessungen Cadmiumsulfid $(C d S)$ und Cadmiumselenid $(C d S e)$ für Phasenumwandlungsexperimente unter Hochdruckbedingungen und ebenfalls als Wiederholungsmessung Nickelsulfid ( NiS ) für Phasenumwandlungsexperimente unter Hochtemperaturbedingungen ausgewählt und ihre Phasenumwandlung per Röntgendiffraktometrie dokumentiert. Die Auswahl der Materialien erfolgte nach der Möglichkeit, ihre Phasenumwandlungsbedingungen mit den vorhandenen experimentellen Mitteln erzeugen zu können. Ebenso spielte die hohe Symmetrie des Kristallgitters bei den ausgewählten Substanzen eine Rolle, um Reflexüberlagerungen auf den Röntgenbildern zu minimieren. Die zur Phasenumwandlung nötigen Drücke wurden in Diamantstempelzellen erzeugt, die Hochtemperaturexperimente wurden in einem Glaskugelofen durchgeführt. Die Messungen erfolgten an den Beamlines BW5, W2 und P02.1 im Hamburger Synchrotronstrahlungslabor (HASYLAB). Ausgangsmaterial war ein Einkristall der Probensubstanz, der im Zuge des Phasenübergangs in ein polykristallines Material mit hoher Vorzugsorientierung der einzelnen Kristallite umgewandelt wurde. Zur Auswertung der Ergebnisse wurden zunächst Polfiguren erstellt, aus denen anschließend die Orientierungsverteilungsfunktion berechnet wurde. Hierfür wurde bei den Hochdruckexperimenten die Harmonische Methode nach Bunge verwendet, bei den Hochtemperaturexperimenten kam das Programm MAUD [43] zum Einsatz. Aus den Polfiguren und der Orientierungsverteilungsfunktion wurden schließlich die Orientierungsbeziehungen bestimmt. 


\section{Theoretische Grundlagen}

\subsection{Kristalline Materialien}

Viele natürliche und industriell gefertigte Materialien sind ganz oder teilweise kristallin. Ein kristallines Material zeichnet sich durch eine dreidimensional periodische Anordnung der Atome in einem Kristallgitter aus. Aufgrund der Periodizität reicht zur Beschreibung der Charakteristika der Kristallstruktur auf atomarer Ebene eine Beschreibung der Charakteristika einer Elementarzelle aus.

Ein einkristallines Material besteht nur aus einem einzigen Kristall, während ein polykristallines Material aus einem Aggregat vieler einzelner Kristallite besteht.

\subsection{Orientierung}

Jedem Kristallit eines Materials ist ein Kristallkoordinatensystem $K_{B}$ zugeordnet, welches in der Regel den Hauptachsen des Kristallgitters entspricht. Die Orientierung eines Kristallits in der Probe wird mit Hilfe des Probenkoordinatensystems $K_{A}$ durch eine Drehung $g$ beschrieben, welche $K_{A}$ in $K_{B}$ überführt (Abbildung 2.1).

$K_{A}$ wird üblicherweise durch die Probengeometrie oder durch ausgezeichnete Richtungen der Probe definiert. Ausgehend von der der Metallographie haben sich folgende Richtungsbezeichnungen durchgesetzt:

- Die x-Richtung wird als Walzrichtung (WR), rolling direction (RD) oder Längsrichtung (LR) bezeichnet.

- Die y-Richtung wird als Querrichtung (QR) oder transversal direction (TD) be- 


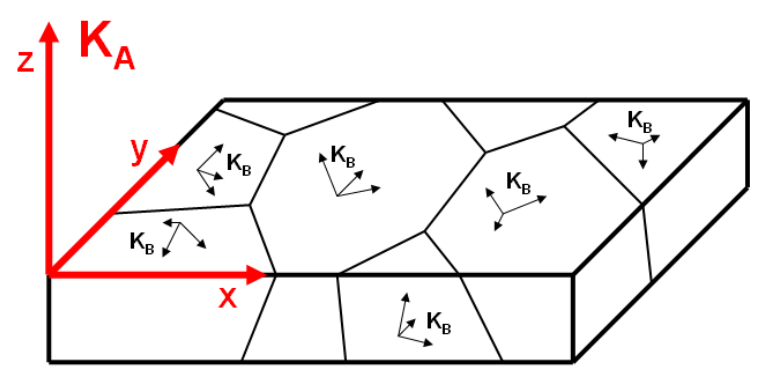

Abbildung 2.1: Probenkoordinatensystem $K_{A}$ und Kristallkoordinatensystem $K_{B}: K_{A} \rightarrow g \rightarrow K_{B}$

zeichnet.

- Die z-Richtung wird als Normalenrichtung (NR) oder normal direction (ND) bezeichnet und steht senkrecht auf der von $\vec{x}$ und $\vec{y}$ aufgespannten Ebene des Probenkoordinatensystems.

Es gibt drei verschiedene Möglichkeiten, die Drehung $g$ in Parametern zu beschreiben: als Eulerwinkel, in $(h k l)[u v w]$-Form und als Matrix dargestellt.

\subsubsection{Eulerwinkel}

Die Beschreibung von $g$ erfolgt durch die Eulerwinkel $\varphi_{1}, \Phi$ und $\varphi_{2}$ :

$$
g=\left\{\varphi_{1}, \Phi, \varphi_{2}\right\}
$$

Die Eulerwinkel sind durch die folgenden Rotationen von $K_{A}$ definiert:

1. Drehung um den Winkel $\varphi_{1}$ um die $z$-Achse. Dies führt zu den Achsenverschiebungen $x \rightarrow x^{\prime}, y \rightarrow y^{\prime}$ und $z \rightarrow z . \varphi_{1}$ ist periodisch in $2 \pi$.

2. Drehung um den Winkel $\Phi$ um die $x^{\prime}$-Achse. Dies führt zu den Achsenverschiebungen $x^{\prime} \rightarrow x^{\prime}, y^{\prime} \rightarrow y^{\prime \prime}$ und $z \rightarrow z^{\prime}$. $\Phi$ ist periodisch in $\pi$.

3. Drehung um den Winkel $\varphi_{2}$ um die $z^{\prime}$-Achse. Dies führt zu den Achsenverschiebungen $x^{\prime} \rightarrow x^{\prime \prime}, y^{\prime \prime} \rightarrow y^{\prime \prime \prime}$ und $z^{\prime} \rightarrow z^{\prime} . \varphi_{2}$ ist periodisch in $2 \pi$. 

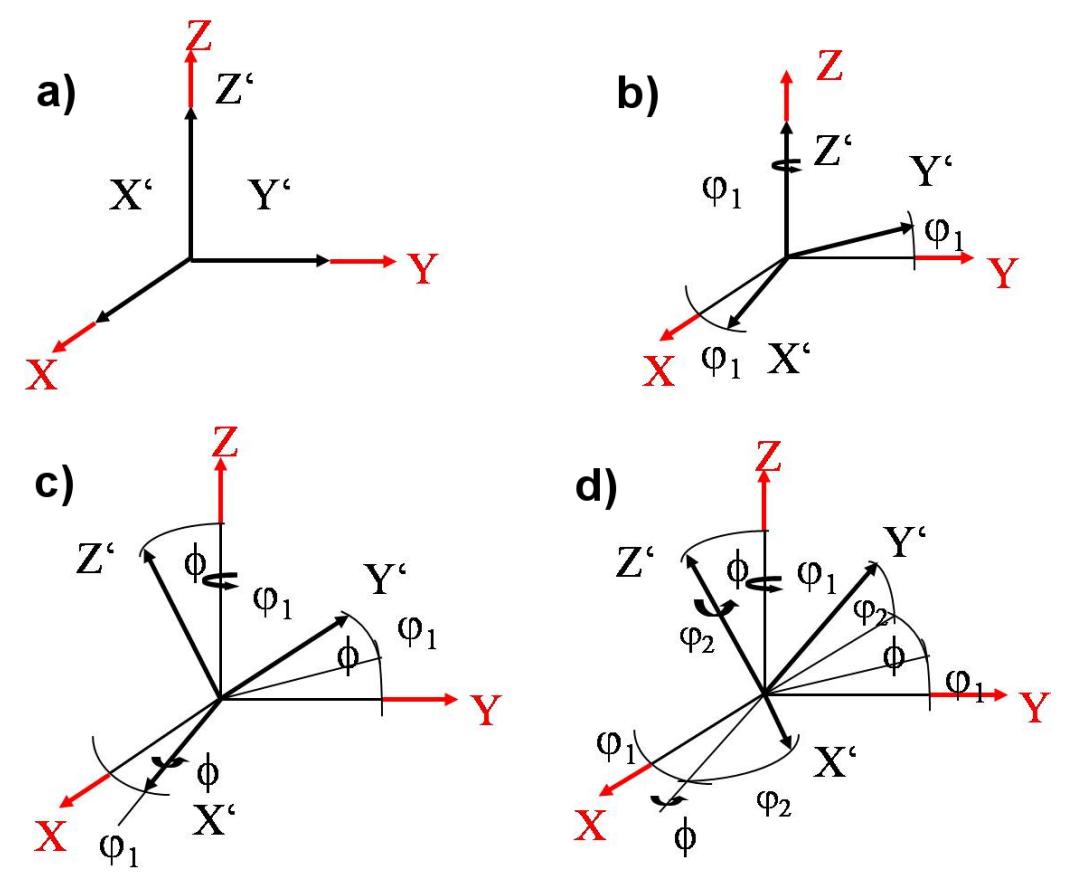

Abbildung 2.2: Rotation des Probenkoordinatensystems um die Eulerwinkel: a) Ausgangszustand, b) Rotation um $\varphi_{1}$, c) Rotation um $\Phi$, d) Rotation um $\varphi_{2}$ (Quelle: H. Klein)

Eine andere Definition der Eulerwinkel beinhaltet eine Rotation um $y^{\prime}$, sie wurde im Rahmen der vorliegenden Arbeit jedoch nicht verwendet.

Die Gesamtmenge aller möglichen Rotationen von $g=\{0,0,0\}$ bis $g=\{2 \pi, \pi, 2 \pi\}$ spannt den Eulerraum oder Orientierungsraum auf, in welchem die Eulerwinkel als kartesische Koordinatenachsen fungieren. Jeder Punkt im Eulerraum beschreibt eine bestimmte Rotation und somit eine bestimmte Orientierung (siehe Abbildung 2.3). Im Fall $\Phi=0$ ist der Eulerraum entartet, da nicht zwischen Rotation um $\varphi_{1}$ und $\varphi_{2}$ unterschieden werden kann.

\subsubsection{Matrixdarstellung}

Die Drehmatrix $g$ beschreibt die Überführung einer Orientierung mit den Koordinaten $x, y, z$ im Probenkoordinatensystem $K_{A}$ in die Koordinaten $x^{\prime}, x^{\prime}, z^{\prime}$ im Kristallkoordinatensystem $K_{B}$. Die Matrixelemente $a_{i j}$ entsprechen den neun Richtungscosinus zwischen den Achsen von $K_{A}$ und $K_{B}$. Hierbei beschreibt die erste Spalte die Längsrichtung $\vec{x}$ $u v w$, die zweite Spalte die Querrichtung $\vec{y}$ rst und die dritte Spalte die Normalenrichtung $\vec{z} h k l$. Per Definition sind die Spaltenvektoren der Matrix orthogonal zueinander 

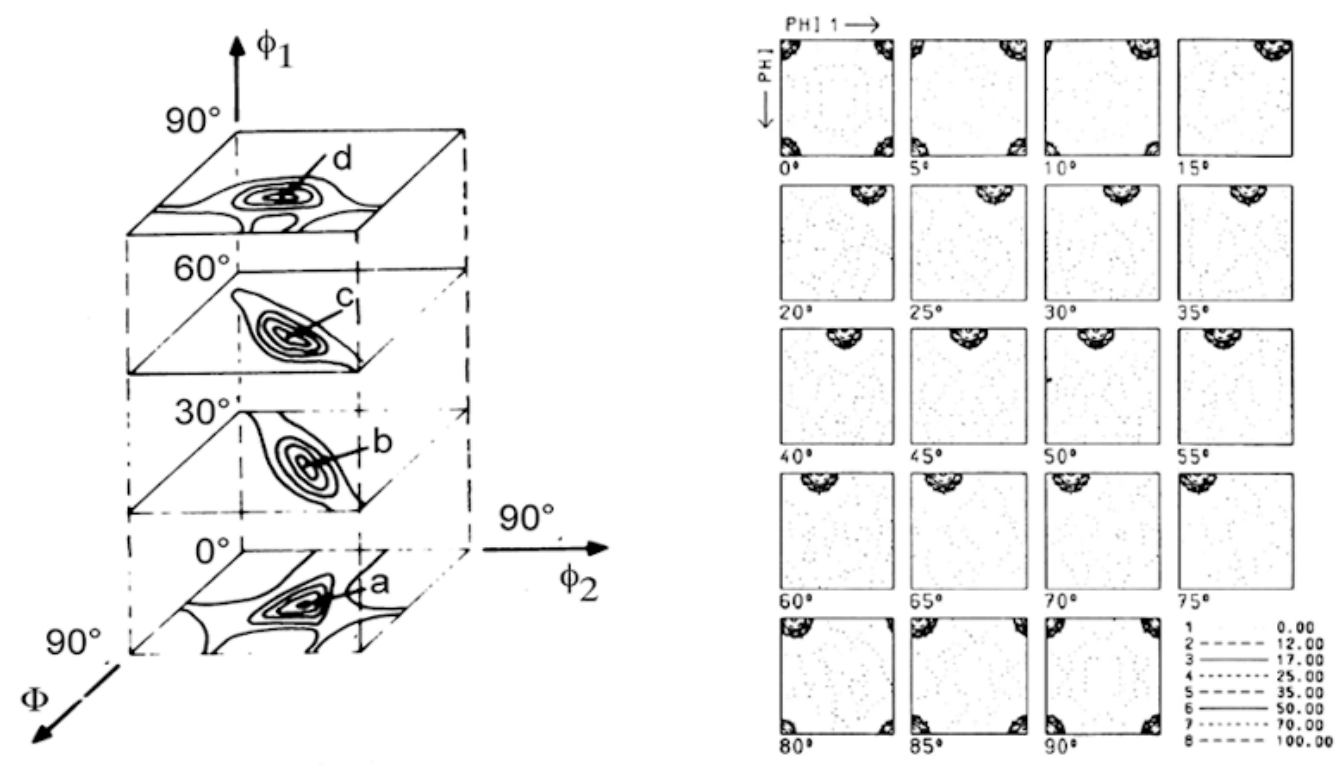

Abbildung 2.3: Links: Schematische Darstellung einer Orienterungsdichteverteilung im Eulerraum in $\varphi_{1-}$ Schnitten. Rechts: Darstellung der Würfellage eines kubischen Materials in $\varphi_{2}$-Schnitten durch den Eulerraum. (Quelle: H. Klein, Vorlesung: Einführung in die mathematische Texturanalyse)

und normiert (Gleichung 2.2).

$$
g=\left[\begin{array}{lll}
u & r & h \\
v & s & k \\
w & t & l
\end{array}\right]=\left[\begin{array}{lll}
a_{11} & a_{12} & a_{13} \\
a_{21} & a_{22} & a_{23} \\
a_{31} & a_{32} & a_{33}
\end{array}\right]=\left[a_{i j}\right]
$$

Die Drehmatrix $g$ lässt sich durch die Eulerwinkel $\varphi_{1}, \Phi$ und $\varphi_{2}$ ausdrücken. Hierfür werden zuerst die Drehmatrizen für die Drehungen um die einzelnen Winkel aufgestellt:

$$
\begin{aligned}
g_{z}\left(\varphi_{1}\right) & =\left(\begin{array}{ccc}
\cos \varphi_{1} & \sin \varphi_{1} & 0 \\
-\sin \varphi_{1} & \cos \varphi_{1} & 0 \\
0 & 0 & 1
\end{array}\right) \\
g_{x^{\prime}}(\Phi) & =\left(\begin{array}{ccc}
1 & 0 & 0 \\
0 & \cos \Phi & \sin \Phi \\
0 & -\sin \Phi & \cos \Phi
\end{array}\right) \\
g_{z^{\prime}}\left(\varphi_{2}\right) & =\left(\begin{array}{ccc}
\cos \varphi_{2} & \sin \varphi_{2} & 0 \\
-\sin \varphi_{2} & \cos \varphi_{2} & 0 \\
0 & 0 & 1
\end{array}\right)
\end{aligned}
$$


Diese drei Komponentendrehmatrizen werden miteinander multipliziert:

$$
g=g_{z}\left(\varphi_{1}\right) \circ g_{x^{\prime}}(\Phi) \circ g_{z^{\prime}}\left(\varphi_{2}\right)
$$

Hieraus ergibt sich schließlich die Drehmatrix $g$ in allgemeiner Form:

$$
g=\left(\begin{array}{ccc}
\cos \varphi_{1} \cos \varphi_{2}-\sin \varphi_{1} \sin \varphi_{2} \cos \Phi & \sin \varphi_{1} \cos \varphi_{2}+\cos \varphi_{1} \sin \varphi_{2} \cos \Phi & \sin \varphi_{2} \sin \Phi \\
-\cos \varphi_{1} \sin \varphi_{2}-\sin \varphi_{1} \cos \varphi_{2} \cos \Phi & -\sin \varphi_{1} \sin \varphi_{2}+\cos \varphi_{1} \cos \varphi_{2} \cos \Phi & \cos \varphi_{2} \sin \Phi \\
\sin \varphi_{1} \sin \Phi & -\cos \varphi_{1} \sin \Phi & \cos \Phi
\end{array}\right)
$$

\subsubsection{Darstellung als $(h k l)[u v w]$}

Die Orientierung eines Kristalls oder die Charakteristika einer Orientierungsverteilung lassen sich eindeutig durch die Angabe einer Fläche und einer senkrecht dazu stehenden Richtung als $g=(h k l)[u v w]$ beschreiben.

$(h k l)$ beschreibt diejenige Netzebene, die in der von Längsrichtung und Querrichtung aufgespannten Fläche liegt. Die Flächennormale zeigt somit in die Normalenrichtung des Probenkoordinatensystems. Die alleinige Angabe von $(h k l)$ weist die Rotation um den Flächennormalenvektor als Freiheitsgrad auf, sodass eine bestimmte Fläche als Gerade im Eulerraum erscheint. Eine Orientierungsverteilung die als Gerade im Eulerraum erscheint, wird Fasertextur genannt. Sie entsteht, wenn die Netzebenen $(h k l)$ einer bestimmten Art rotationssymmetrisch um eine feste Probenrichtung verteilt sind. Abbildung 2.4 zeigt die schematische Darstellung einer Fasertextur im Eulerraum als $\varphi_{2}$-Schnitt.

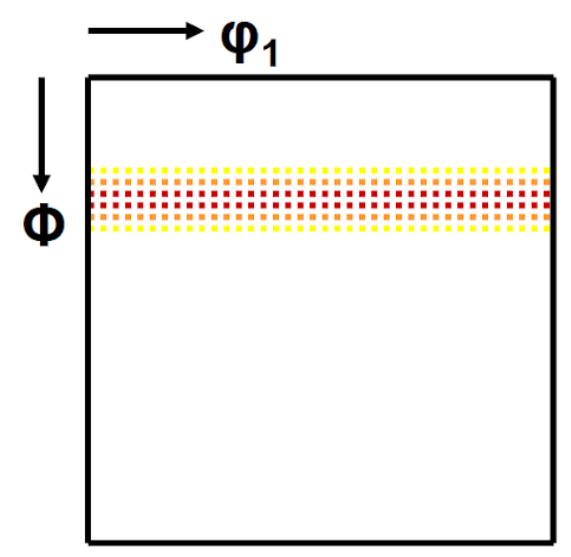

Abbildung 2.4: Schematische Darstellung einer Fasertextur im Eulerraum als $\varphi_{2}$-Schnitt. 
[uvw] beschreibt die Kristallrichtung, die in Richtung der Längsrichtung des Probenkoordinatensystems zeigt. Für ein bestimmtes $(h k l)$ können nur bestimmte [uvw] angenommen werden. [uvw] erscheint im Eulerraum als Punkt auf der dazugehörigen $(h k l)$ Gerade. 


\subsection{Der Texturbegriff}

Der Begriff Textur beschreibt die Gesamtheit der Einzelorientierungen aller Kristallite in einem gemessenen Probenvolumen. Als Maß für die Stärke oder Schärfe einer Textur gilt die Regellosigkeit: Sind die Orientierungen der Kristallite statistisch gleich verteilt, wird von einer 1-fach regellosen Textur gesprochen. In den allermeisten polykristallinen Materialien weisen die Kristallite jedoch bevorzugte Orientierungen auf, welche durch den Bildungs-, Herstellungs- und Bearbeitungsprozess des Materials entstehen. So neigen zum Beispiel plättchenförmige Kristallite dazu, sich senkrecht zur Richtung des stärksten Druckes anzuordnen. Solche Materialien weisen einen Regellosigkeitsfaktor von mehr als 1 auf: Je höher der Regellosigkeitsfaktor, desto stärker ist die Vorzugsorientierung ausgeprägt. Man spricht von einer n-fach regellosen Textur. Die Vorzugsorientierung sorgt für eine Richtungsabhängigkeit der makroskopischen Materialeigenschaften, welche für die Werkstofftechnik eine bedeutende Rolle spielt. Je stärker die Vorzugsorientierung, desto stärker nähern sich die Materialeigenschaften denen eines Einkristalls an, der nur eine einzige Orientierung aufweist.

Zur vollständigen quantitativen Beschreibung der Textur wird die Orientierungsverteilungsfunktion (Orientation Distribution Function, ODF) $f(g)$ verwendet. Sie gibt denjenigen Volumenanteil $d V$ der Kristallite an, deren Orientierung $g$ innerhalb eines bestimmten Orientierungsintervalls $d g$ liegt und ordnet damit allen Einzelorientierungen einen Funktionswert im Eulerraum zu (Gleichung 2.8) [13].

$$
\frac{d V(g)}{V}=f(g) d g
$$

Analog zum Regellosigkeitsbegriff ist die ODF einer regellosen Textur auf 1 normiert und der Wert von $f(g)$ ergibt sich als ein Vielfaches des Werts für eine regellose Textur. Es somit gilt die Normierungsbedingung Gleichung 2.9:

$$
f_{\text {regellos }}=\oint f(g) d g=1
$$

Es ist meist nicht möglich, die ODF direkt zu messen. Stattdessen wird die Poldichteverteilung verschiedener Netzebenen der Kristallite bestimmt und daraus die ODF berechnet. Verbreitete Verfahren zur Messung der Poldichteverteilung sind die Röntgenbeugung, die Neutronenbeugung und die Elektronenbeugung. Im Rahmen der vorliegenden Arbeit wurde ausschließlich mit Röntgenbeugungsmethoden gearbeitet. 


\subsubsection{Die Bragg-Bedingung}

Der Abstand $d$ zwischen zwei Netzebenen eines Kristallgitters liegt typischerweise in einer Größenordnung zwischen $10^{-9}$ und $10^{-10} \mathrm{~m}$. Um Informationen über die Kristallstruktur auf atomarer Ebene zu bekommen, muss mit Strahlung mit einer ähnlichen Wellenlänge gearbeitet werden. Der Vorteil der Röntgenbeugung ist, dass sie zerstörungsfrei ist, nicht zwangsweise einer aufwendigen Probenpräparation bedarf und im Gegensatz zur Neutronenbeugung auch keine für den Anwender gefährliche Strahlenbelastung der Probe auftritt.

Trifft Röntgenstrahlung unter dem Einfallswinkel $\vartheta$ auf ein kristallines Material, wird diese an den Netzebenen nach dem Reflexionsgesetz im gleichen Winkel gebeugt. Beträgt der Lauflängenunterschied $2 \cdot d \cdot \sin \vartheta$ zwischen an verschiedenen Ebenen der gleichen Netzebenenschar gebeugten Wellenfronten ein ganzzahliges Vielfaches $n$ der Wellenlänge $\lambda$, ist eine positive Interferenz gegeben und der Detektor empfängt ein Signal. Für einen bestimmten Netzebenenabstand ist diese Bedingung nur unter einem charakteristischen Wert für $\vartheta$ gegeben (Bragg-Bedingung (Gleichung 2.10), siehe auch Abbildung 2.5) [9]:

$$
n \cdot \lambda=2 \cdot d \cdot \sin \vartheta
$$

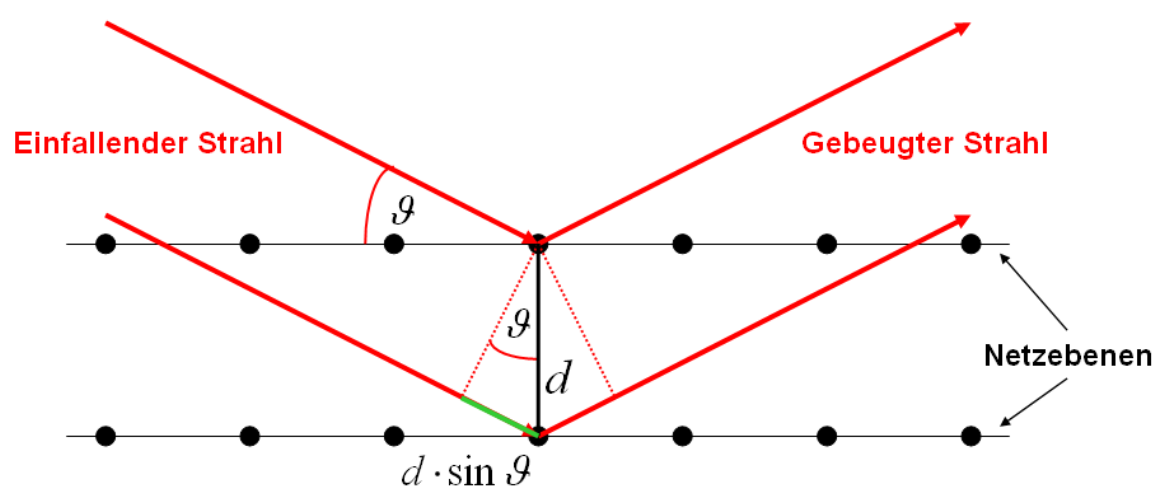

Abbildung 2.5: Graphische Darstellung der Bragg-Bedingung. $\vartheta=$ Einfalls- und Ausfallswinkel, $d=$ Netzebenenabstand

Fällt ein monochromatischer Röntgenstrahl mit der Wellenlänge $\lambda$ auf eine kristalline Probe, reflektieren die Netzebenen derjenigen Kristalle den Strahl, für welche die Bragg-Bedingung unter den gegebenen Umständen erfüllt ist. Die Schar der reflektierten Photonen einer Netzebenenschar bildet einen Kegel mit dem Öffnungswinkel $2 \vartheta$ um den Primärstrahl, der auf einem Leuchtschirm oder Flächendetektor als Kreis sichtbar 
wird. Bei einer einfach regellosen Textur erscheint dieser Kreis homogen. Liegt eine Vorzugsorientierung der Kristallite vor, zeigen sich Knoten höherer Intensität entlang des Kreises. Einkristalle zeigen scharfe, punktförmige Reflexe (Abbildung 2.6).

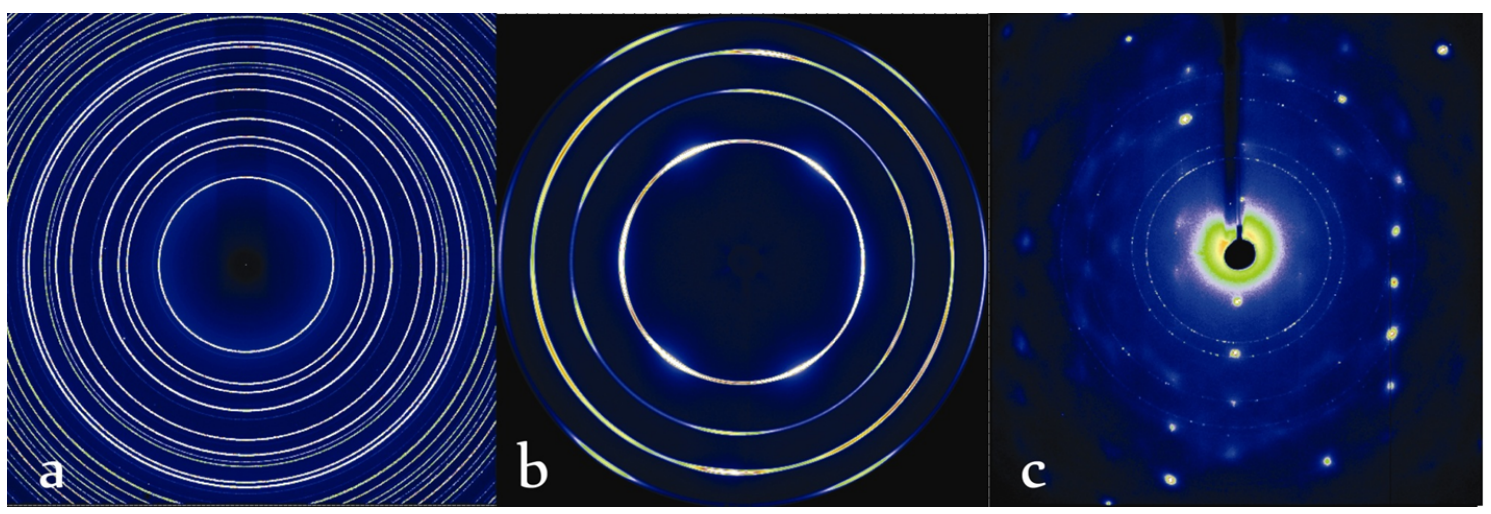

Abbildung 2.6: Detektorbilder verschiedener Materialien. a) $\mathrm{Al}_{2} \mathrm{O}_{3}$ mit einfach regelloser Orientierung, b )Stahl mit Vorzugsorientierung c) Citrin-Einkristall (Quelle: I. Janßen) 


\subsection{Darstellung von Polfiguren}

\subsubsection{Definition einer Polfigur}

Eine Polfigur $P_{h}(\vec{y})$ ist die Verteilung einer Netzebenenart $\{h k l\}$ im Probenkoordinatensystem $K_{A}$. Sie ist als eine zweidimensionale Dichtefunktion der Flächenpole einer bestimmten Netzebenenart $\{h k l\}$ im gemessenen Probenvolumen definiert [86]. Da eine direkte Messung der dreidimensionalen ODF $f(g)$ meist nicht möglich ist, muss sie aus den gemessenen Polfiguren berechnet werden.

Im durch ausgezeichnete Probenrichtungen aufgespannten Probenkoordinatensystem $K_{A}$ wird eine beliebige Probenrichtung $\vec{y}$ durch die Polarkoordinaten $\{\alpha, \beta\}$ beschrieben. Hierbei läuft $\alpha$ vom Zenit der Lagenkugel zum Äquator und $\beta$ gegen den Uhrzeigersinn um den Äquator herum. Die Winkel $\alpha$ und $\beta$ werden auch als Polfigurwinkel bezeichnet.

\subsubsection{Das Wulffsche Netz}
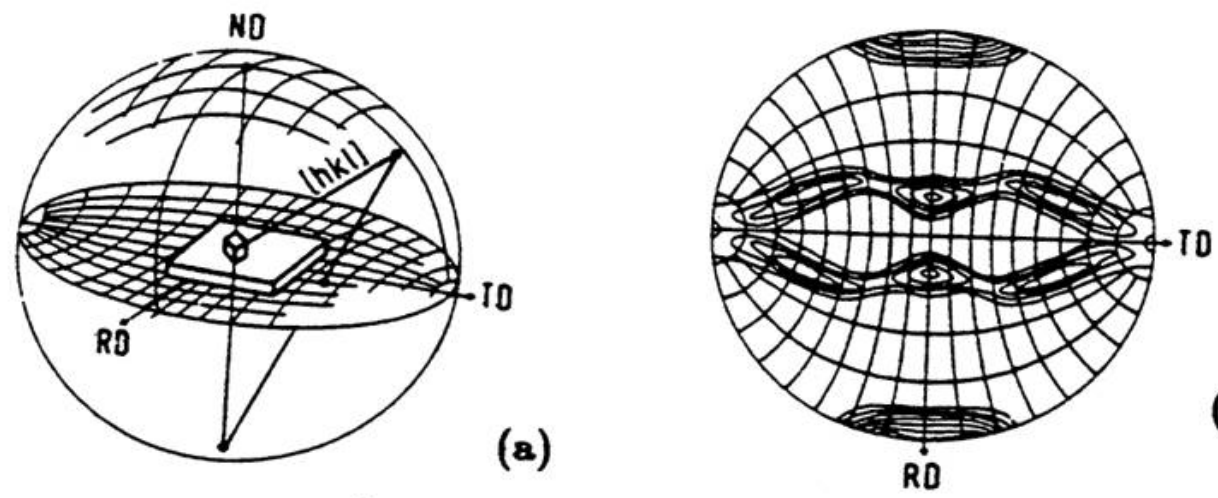

(b)

Abbildung 2.7: a) Schematische Darstellung a) der Lagenkugel, b) einer Polfigur im Wulffschen Netz. (Quelle: H. Klein)

Polfiguren werden als winkeltreue stereographische Projektion im Wulffschen Netz dargestellt [91]. Hierzu wird eine Probenrichtung $\vec{y}=\{\alpha, \beta\}$ von ihrer Position auf der Lagenkugel auf die Äquatorebene selbiger projiziert. Als Lage im Wulffschen Netz gilt der Durchstichpunkt der Verbindungslinie von der Lagenkugelposition von $\vec{y}$ zum entgegengesetzten Pol der Lagenkugel. Bei der Darstellung einer Polfigur entspricht $\alpha$ der 
Winkeldistanz zwischen dem Flächenpol und dem Nord- bzw. Südpol der Lagenkugel. $\alpha$ kann Werte zwischen $0^{\circ}(\mathrm{Pol})$ und $90^{\circ}$ (Äquator) annehmen. $\beta$ wird entgegen dem Uhrzeigersinn am Äquator abgetragen, wobei $0^{\circ}$ am rechten Rand liegt. Der Wertebereich von $\beta$ reicht von $0^{\circ}$ bis $360^{\circ}$. Winkel zwischen zwei Flächenpolen oder Intensitätsmaxima werden über die Großkreise abgelesen. Die Lage des Wulffschen Netzes wird durch das Probenkoordiantensystem $K_{A}$ festgelegt. Konventionell zeigt die Längsrichtung nach oben, die Querrichtung in Richtung des Nullpunkts des $\beta$-Kreises und die Normalenrichtung senkrecht aus der Darstellung heraus. Abbildung 2.7 zeigt eine schematische Darstellung des Wulffschen Netzes mit allen relevanten Richtungen und Winkeln. 


\subsection{Berechnung der ODF aus Polfiguren}

Es gibt eine Vielzahl von Methoden zur Berechnung der ODF. Im Rahmen der vorliegenden Arbeit wurden zwei von ihnen angewandt: Die Harmonische Methode und die E-WIMV-Methode.

\subsubsection{Die Fundamentalgleichung der Texturanalyse}

Das Kristallkoordinatensystem $K_{B}$ wird in der Regel durch die Hauptachsen des Kristallsystems der Kristallite aufgespannt. Eine beliebige Kristallrichtung $\vec{h}$ wird durch die Polarkoordinaten $\{\theta, \gamma\}$ ausgedrückt. Die Probenrichtung $\vec{y}=\{\alpha, \beta\}$ wird über das Wulffsche Netz ausgedrückt. Hier läuft $\alpha$ vom Zenit der Lagenkugel zum Äquator und $\beta$ gegen den Uhrzeigersinn um den Äquator herum.

Der Mittelwert der Orientierungsverteilungsfunktion über alle Orientierungen ist die Achsenverteilungsfunktion der Richtungen $\vec{h}=(\theta, \gamma)$ und $\vec{y}=(\alpha, \beta)$. Die Achsenverteilung lässt sich als Integral der $\operatorname{ODF} f(g)$ berechnen, wenn alle Orientierungen $g$ betrachtet werden, für die $\vec{h} \| \vec{y}$ gilt (Gleichung 2.11):

$$
A(\vec{h}, \vec{y})=\frac{1}{2 \pi} \int_{h \| y} f(g) d \psi
$$

Wird die Kristallrichtung $\vec{h}$ festgehalten und somit nur eine einzige Netzebenenschar $(h k l)$ betrachtet, lässt sich $A(\vec{h}, \vec{y})$ durch die Poldichte $P_{h}(\alpha, \beta)$ ausdrücken. Somit ergibt sich die Poldichte folgendermaßen (Gleichung 2.12):

$$
P_{h k l}(\alpha, \beta)=\frac{1}{2 \pi} \int_{h \| y} f(g) d \psi
$$

Gleichung 2.12 wird auch die Fundamentalgleichung der Texturanalyse genannt [14]. Um die ODF aus den gemessenen Polfiguren zu erhalten, muss die Fundamentalgleichung umgekehrt werden. Dieses Verfahren wird Polfigurinversion genannt. Um das Integral zu lösen gibt es verschiedene Methoden, von denen zwei im Rahmen der vorliegenden Arbeit verwendet wurden. 


\subsubsection{Die Harmonische Methode}

Bei der harmonischen Methode, auch bekannt als Reihenentwicklungsmethode nach Bunge ([13], [14]), wird die Orientierungsverteilungsfunktion $f(g)$ als Reihe symmetrisch verallgemeinerter Kugelfunktionen $\dot{T}_{l}^{\mu \nu}(g)$ und den Texturkoeffizienten $C_{l}^{\mu \nu}$ beschrieben.

$$
f(g)=\sum_{l=0}^{L_{\max }} \sum_{\mu=1}^{M(l)} \sum_{\nu=1}^{N(l)} C_{l}^{\mu \nu} \cdot \dot{\bar{T}}_{l}^{\mu \nu}(g)
$$

: steht hierbei für die Invarianz der Funktion gegenüber der Kristallsymmetrie, . für die Invarianz gegenüber der Probensymmetrie. Somit ist es je nach Proben- und Kristallsymmetrie möglich, den dargestellten Teil des Eulerraums zu reduzieren und trotzdem alle Informationen zu erhalten. Der Parameter $l$ beschreibt den Reihenentwicklungsgrad der Funktion. Bei einem realen Experiment steht nur eine begrenzte Anzahl gemessener Polfiguren zur Verfügung, deren Anzahl in Abhängigkeit von Proben- und Kristallsymmetrie den maximalen Reihenentwicklungsgrad $L_{\max }$ bestimmt. $\mu(l)$ und $\nu(l)$ stellen die Laufindizes der Funktionen der Kristall- bzw. der Probensymmetrie dar. Der maximale Wert $M(l)$ des Index $\mu(l)$ entspricht der Zahl der linear unabhängigen Symmetrieoperationen im Kristallkoordinatensystem, der maximale Wert $N(l)$ des Index $\nu(l)$ entspricht der Zahl der linear unabhängigen Symmetrieoperationen im Probenkoordinatensystem. Die Entwicklungskoeffizienten $C_{l}^{\mu \nu}$ lassen sich nicht direkt aus den gemessenen Polfiguren berechnen. Sie müssen über die Achsenverteilungsfunktion (Gleichung 2.11) und die Fundamentalgleichung der Texturanalyse (Gleichung 2.12) bestimmt werden. Durch das Einsetzen von Gleichung 2.13 in Gleichung 2.12 erhält man:

$$
A(\vec{h}, \vec{y})=\frac{1}{2 \pi} \sum_{l=0}^{L_{\max }} \sum_{\mu=1}^{M(l)} \sum_{\nu=1}^{N(l)} C_{l}^{\mu \nu} \int_{h \| y} T_{l}^{\mu \nu} d \psi^{\prime}
$$

mit

$$
A(\vec{h}, \vec{y})=\frac{1}{2 \pi} \int_{h \| y} f(g) d \psi=\sum_{l=0}^{L_{\max }} \sum_{\mu=1}^{M(l)} \sum_{\nu=1}^{N(l)} \frac{4 \pi}{2 l+1} C_{l}^{\mu \nu} \dot{k}_{l}^{* \mu}(\vec{h}) \dot{k}_{l}^{\nu}(\vec{y})
$$

Hierbei stellen $\dot{k}_{l}^{* \mu}(\vec{h})$ die komplex konjugierten symmetrischen Kugelflächenfunktionen der Kristallrichtung $\vec{h}$ und $\dot{k}_{l}^{\nu}(\vec{y})$ die symmetrischen Kugelflächenfunktionen der Probenrichtung $\vec{y}$ dar. Sie ergeben sich aus dem Additionstheorem für die verallgemeinerten 
Kugelfunktionen $T_{l}^{m n}(g)$ :

$$
\begin{array}{r}
T_{l}^{m n}(g)=T_{l}^{m n}\left(\varphi_{1}, \Phi, \varphi_{2}\right)=\sum_{m} \sum_{n} \sum_{s} T_{l}^{m s}\left(g_{2}\right) \cdot T_{l}^{s n}\left(g_{1}\right) \\
T_{l}^{0 n}\left(\varphi_{1}, \Phi\right)=\sqrt{\frac{4 \pi}{2 l+1}} k_{l}^{n}(\Phi, \gamma) \\
T_{l}^{m 0}\left(\Phi, \varphi_{2}\right)=\sqrt{\frac{4 \pi}{2 l+1}} k_{l}^{* m}(\Phi, \beta)
\end{array}
$$

Dies führt zu

$$
\int_{h \| y} T_{l}^{\mu \nu} d \psi=\frac{8 \pi^{2}}{2 l+1} \dot{k}_{l}^{* \mu}(\vec{h}) \dot{k}_{l}^{\nu}(\vec{y})
$$

Dies wird in Gleichung 2.14 eingesetzt, um Gleichung 2.15 zu erhalten. Wird $\vec{h}$ konstant gehalten, ergibt sich für die Fundamentalgleichung 2.12 Folgendes:

$$
P_{h k l}(\alpha, \beta)=\sum_{l=0}^{L_{\max }} \sum_{\nu=1}^{N(l)} F_{l}^{\nu}(h k l) \cdot \dot{k}_{l}^{\nu}(\alpha, \beta)
$$

Damit setzen sich die Reihenentwicklungskoeffizienten $F_{l}^{\nu}(h k l)$ aus folgenden Komponenten zusammen:

$$
F_{l}^{\nu}(h k l)=\frac{4 \pi}{2 l+1} \sum_{\mu=1}^{M(l)} C_{l}^{\mu \nu} \cdot \dot{k}_{l}^{* \mu}(\theta, \gamma)
$$

Gleichung 2.21 stellt ein lineares Gleichungssystem für jedes Indexpaar $l$ und $\nu$ mit der Anzahl der unabhängigen Funktionen der Kristallsymmetrie $M(l)$ als Variable von $C_{l}^{\mu \nu}$ dar. Dieses Gleichungssystem kann eindeutig gelöst werden, wenn mindestens so viele Gleichungen wie Variablen, d.h. experimentelle Polfiguren (hkl), bekannt sind. Die Werte der F-Koeffizienten $F_{l}^{\nu}(h k l)$ können dabei aus den experimentellen Polfiguren durch Inversion der Gleichung 2.20 und die Integration über alle Probenrichtungen $\vec{y}=(\alpha, \beta)$ berechnet werden:

$$
F_{l}^{\nu}(h k l)_{\exp }=\int_{\alpha=0}^{\pi} \int_{\beta=0}^{2 \pi} P_{h k l}(\alpha, \beta) \cdot \dot{k}_{l}^{* \nu}(\alpha, \beta) \sin \alpha d \alpha d \beta
$$

Um den Einfluss von Messfehlern zu minimieren, wird Gleichung 2.21 durch das leastsquares-Verfahren mit den in Gleichung 2.22 bestimmten $F_{l}^{\nu}(h k l)_{\text {exp }}$ und folgender Be- 
dingung gelöst:

$$
\sum_{(h k l)}=\left[F_{l}^{\nu}(h k l)_{\exp }-\frac{4 \pi}{2 l+1} \sum_{\mu=1}^{M(l)} C_{l}^{\mu \nu} \cdot \dot{k}_{l}^{* \mu}(\theta, \gamma)\right]^{2}=\text { Min }
$$

Mit den so erhaltenen C-Koeffizienten $C_{l}^{\mu \nu}$ lassen sich die berechneten F-Koeffizienten $F_{l}^{\nu}(h k l)_{c a l c}$ berechnen. Somit lässt sich über die Fundamentalgleichung 2.12 jede beliebige Polfigur der vorliegenden Messung berechnen. So können unvollständig gemessene Polfiguren vervollständigt und nicht messbare Polfiguren dargestellt werden. Über Gleichung 2.13 lässt sich aus den nun bekannten C-Koeffizienten die ODF berechnen. Abbildung 2.8 stellt den Berechnungspfad schematisch dar.

Bei einer Messung mit Röntgenstrahlung oder Neutronen kann nicht zwischen einer Netzebene $(h k l)$ und ihrer Rückseite $(\bar{h} \bar{k} \bar{l})$ unterschieden werden (Friedelsche Regel). Deshalb ist eine gemessene Polfigur immer eine Überlagerung beider Möglichkeiten:

$$
\tilde{P}_{h k l}(\alpha, \beta)=\frac{1}{2}\left[P_{h k l}(\alpha, \beta)+P_{\bar{h} \bar{k} \bar{l}}(\alpha, \beta)\right]
$$

Hieraus ergibt sich aus den dazugehörigen F-Koeffizienten $\tilde{F}_{l}^{\nu}(h k l)$ folgende Relation:

$$
\dot{k}_{l}^{* \mu}\left(\theta_{\bar{h} \bar{k} \bar{l}}, \gamma_{\bar{h} \bar{k} \bar{l}}\right)=(-1)^{l} \cdot \dot{k}_{l}^{* \mu}\left(\theta_{h k l}, \gamma_{h k l}\right)
$$

Somit wird Gleichung 2.21 zu Gleichung 2.26:

$$
\tilde{F}_{l}^{\nu}(h k l)=\frac{4 \pi}{2 l+1} \sum_{\mu=1}^{M(l)} C_{l}^{\mu \nu} \cdot \frac{1}{2}\left[1+(-1)^{l}\right] \dot{\dot{k}}_{l}^{* \mu}\left(\theta_{h k l}, \gamma_{h k l}\right)
$$

Dies bedeutet, dass für ungerade Reihenentwicklungsgrade $l$ die Gleichung Null wird und somit aus gemessenen Polfiguren nur C-Koeffizienten mit geraden $l$-Werten berechnet werden können. Dies führt zur geraden oder reduzierten ODF $\tilde{f}(g)$ und ihrem in den Polfiguren unsichtbaren Gegenpart $\tilde{\tilde{f}}(g)$. Während der Berechnung der ODF können durch die Iteration negative oder falsche positive Werte (Geister) auftreten. Da es keine negativen Volumenanteile von Kristalliten mit Orientierungen $g$ geben kann, wird hier die Positivitätsbedingung verwendet:

$$
P_{h k l}(\alpha, \beta) \geq 0
$$




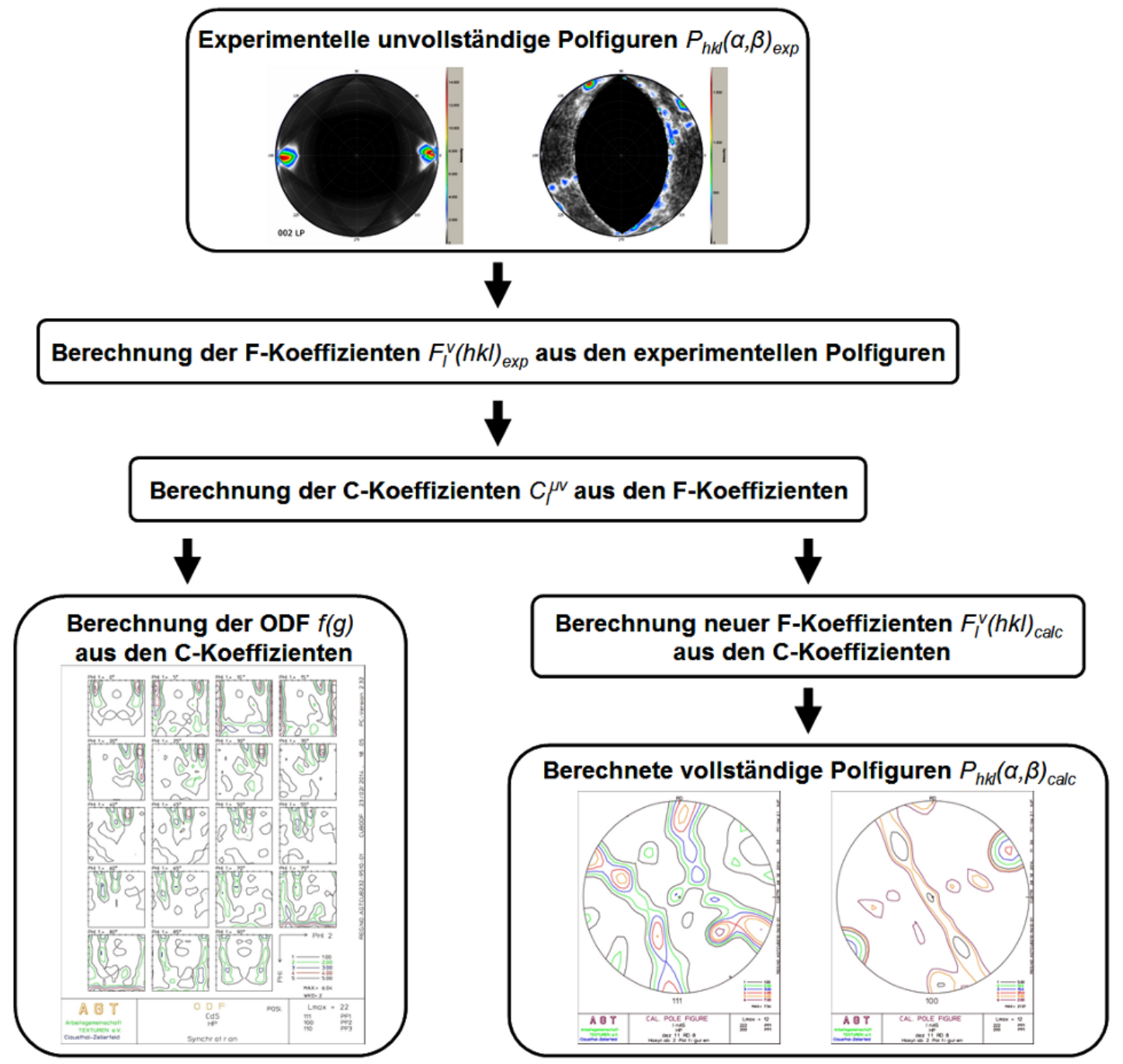

Abbildung 2.8: Schema des Wegs zur Berechnung der ODF und der vollständigen Polfiguren nach der Harmonischen Methode. (nach L. Raue [56])

Wird Gleichung 2.13 anhand der Werte von $l$ in ihren geraden Teil $\tilde{f}(g)$ und ihren ungeraden Teil $\tilde{\tilde{f}}(g)$ zerlegt, gilt für die gesamte ODF:

$$
f(g)=\tilde{f}(g)+\tilde{\tilde{f}}(g) \geq 0
$$

Da er in den Polfiguren unsichtbar ist, wirkt es auf den ersten Blick, als könnte der ungerade Teil der ODF $\tilde{\tilde{f}}(g)$ willkürlich bestimmt werden, er ist jedoch durch die Positivitätsbedingung Gleichung 2.28 eingeschränkt. Eine Näherung für $\tilde{\tilde{f}}(g)$ kann folgenderweise erhalten werden: 
Es wird angenommen, dass bereits eine $n$-te Näherung für $\tilde{\tilde{f}}(g)$ existiert:

$$
{ }^{(n)} \tilde{\tilde{f}}(g)=\sum_{l \mu \nu} C_{l}^{\mu \nu} \cdot \dot{\bar{T}}_{l}^{\mu \nu}(g)
$$

womit als $n$-te Näherung für die komplette ODF gilt:

$$
{ }^{(n)} f(g)=\tilde{f}(g)+{ }^{(n)} \tilde{\tilde{f}}(g)
$$

Falls diese $n$-te Näherung der kompletten ODF immer noch negative Bereiche aufweist, werden diese durch eine Hilfsfunktion ersetzt:

$$
\begin{array}{r}
{ }^{(n+1)} \hat{f}(g)=0 \text { wenn }^{(n)} f(g)>0 \\
(n+1) \hat{f}(g)=-{ }^{(n)} f(g) \text { wenn }^{(n)} f(g)<0
\end{array}
$$

Anschließend wird diese Hilfsfunktion ${ }^{(n+1)} \hat{f}(g)$ durch eine ungerade Funktion ${ }^{(n+1)} \tilde{\tilde{f}}(g)$ unter folgender Bedingung angenähert:

$$
\oint\left[(n+1) \hat{f}(g)-^{(n+1)} \tilde{\tilde{f}}(g)\right]^{2} d g=\text { Min } .
$$

Dieser Vorgang wird wiederholt bis die Näherung keine negativen Bereiche mehr aufweist. Somit gilt für die endgültige Näherung der kompletten ODF:

$$
f(g)=\tilde{f}(g)+\sum_{n=1}^{n_{\max }}(n) \tilde{\tilde{f}}(g)
$$

Aufgrund experimenteller Bedingungen wie zum Beispiel der Geometrie des Versuchsaufbaus können oft keine kompletten Polfiguren gemessen werden. Es ist jedoch möglich, aus der Gesamtheit der gemessenen Polfiguren die fehlenden Informationen für die nicht gemessenen Bereiche einzelner Polfiguren per Iteration anzunähern. Die nicht gemessenen Bereiche werden auf Null gesetzt. Es gilt also die Positivitätsbedingung (Gleichung 2.27) für alle Polfiguren $h k l$ und alle Winkel. Die aus der endlichen Anzahl gemessener Polfiguren resultierende Fehlergröße bei der Berechnung der C-Koeffizienten wird hierdurch verringert, sodass die Berechnung auch bei wenigen Polfiguren zu einem hohen Reihenentwicklungsgrad geführt werden kann.

Der Fehler $\Delta P_{h}$ lässt sich durch den Vergleich der experimentellen mit den berechneten 
Polfiguren abschätzen. Hierzu wird eine Differenzpolfigur gebildet:

$$
\Delta P_{h k l}=P_{h k l}(\alpha, \beta)_{e x p}-P_{h k l}(\alpha, \beta)_{c a l c}
$$

Im Idealfall hat diese Differenzpolfigur überall den Wert Null.

Der ideale Reihenentwicklungsgrad einer ODF-Berechnung hängt von der Anzahl der gemessenen Polfiguren, der Qualität ebendieser und der Stärke der Textur ab. Die Konvergenz der ODF lässt sich anhand der Mittelwerte der C-Koeffizienten abschätzen:

$$
C_{l}=\left|C_{l}^{\bar{\mu} \nu}\right|=\frac{1}{M(l) \cdot N(l)} \sum_{\mu=1}^{M(l)} \sum_{\nu=l}^{N(l)}\left|C_{l}^{\mu \nu}\right|
$$

Der Fehler $\Delta C_{l}$ lässt sich aus der Reihenentwicklung der Differenzpolfiguren $\Delta P_{h k l}$ bestimmen und für ihn gilt nach Gleichung 2.37:

$$
\Delta C_{l}=\left|\Delta \bar{C}_{l}^{\mu \nu}\right|=\frac{1}{M(l) \cdot N(l)} \sum_{\mu=1}^{M(l)} \sum_{\nu=l}^{N(l)}\left|\Delta C_{l}^{\mu \nu}\right|
$$

Eine Reihenentwicklung hat Konvergenz erreicht, wenn die Mittelwerte der C-Koeffizienten die Größenordnung des Fehlers erreicht haben. Dies geschieht für schwache Texturen schneller als für starke Texturen.

\subsubsection{Die E-WIMV-Methode}

Die Williams-Imhof-Matthies-Vinel-Methode (WIMV) [45], [90], [34] ist eine iterative Methode zur Berechnung der ODF, die auf der numerischen Verfeinerung von $f(g)$ im Schritt $n+1$ basiert (Gleichung 2.38).

$$
f_{n+1}(g)=f_{n}(g) \cdot\left(\frac{\prod_{h=1}^{N} \prod_{m=1}^{M_{h}} P_{h}^{e x p}(y)}{\prod_{h=1}^{N} \prod_{m=1}^{M_{h}} P_{h}^{c a l c, n}(y)}\right)^{\frac{1}{N M_{h}}}
$$

Hierbei bezeichnet $P_{h}^{e x p}(y)$ die experimentellen Polfiguren, $P_{h}^{c a l c, n}(y)$ die aus der $n$-ten Iteration berechneten Polfiguren, $N$ bezeichnet die Anzahl der gemessenen Polfiguren, $M_{h}$ die Multiplizität des jeweiligen Flächenpols. Zur Berechnung wird die ODF in eine endliche Anzahl von Zellen aufgeteilt, denen jeweils ein diskreter Wert zugeordnet wird. 
Die Größe dieser Zellen bestimmt die Auflösung der ODF.

Um die Ergebnisse der ODF-Berechnung durch die WIMV-Methode im Zusammenhang mit einer Rietveld-Verfeinerung [58] zu verbessern und Fehler durch Unregelmäßigkeiten bei der Polfigurmessung auszugleichen, wurde die E-WIMV-Methode entwickelt. Das E steht für den Zusatz 'Entropiemodifiziert' und es wird ein zusätzlicher Parameter $c$ eingeführt, welcher bei der Berechnung der ODF als Gewichtungsfaktor dient. $c$ ist abhängig von der Intensität und dem Überlappungsfaktor der gemessenen Reflexe. Hiermit wird Gleichung $2.38 \mathrm{zu}$ Gleichung 2.39:

$$
f_{n+1}(g)=f_{n}(g) \cdot\left(\frac{\prod_{h=1}^{N} \prod_{m=1}^{M_{h}} P_{h}^{e x p}(y)}{\prod_{h=1}^{N} \prod_{m=1}^{M_{h}} P_{h}^{c a l c, n}(y)}\right)^{\frac{c}{N M_{h}}}
$$

Die E-WIMV-Methode findet im Programm MAUD [43] Anwendung, das für die Auswertung eines Teils der Hochtemperatur-Messdaten verwendet wurde, und in Kapitel 6.2 näher beschrieben wird. 


\subsection{Phasenumwandlungen}

Der Gleichgewichtsabstand zwischen Atomen unter den gegebenen Umgebungsbedingungen entspricht der geringsten potentiellen Energie. Auf ein kristallines Material bezogen bedeutet dies, dass bei gegebenen Druck- und Temperaturbedingungen immer die Phase mit der niedrigsten Gibbs'schen freien Energie $G$ stabil ist:

$$
G=H-T \cdot S
$$

Mit der Enthalpie $H$, der Entropie $S$ und der Umgebungstemperatur T. Die Enthalpie $H=E+p \cdot V$ enthält die Terme für die innere Energie $E$, den Umgebungsdruck $p$ und das Einheitszellenvolumen $V$. Somit ist $G$ sowohl vom Druck als auch von der Temperatur abhängig:

$$
G=E-T \cdot S+p \cdot V
$$

Aufgrund des Strebens eines Systems nach dem stabilsten energetischen Zustand laufen alle Reaktionen in Richtung des kleinstmöglichen Wertes für die Gibbs'sche freie Energie. Um den energetisch günstigsten Zustand zu bestimmen, wird der Unterschied $G_{2}-G_{1}=$ $\Delta G$ betrachtet:

$$
\Delta G=\Delta E-T \cdot \Delta S+p \cdot \Delta V
$$

Nimmt $\Delta G$ einen negativen Wert an, ist die ursprüngliche Phase nicht mehr stabil und es kommt zu einer Phasenumwandlung.

Für druckinduzierte Phasenübergänge gelten oft die Neuhaus'schen Regeln [51]:

1. Druck-Koordinationsregel: Die Koordinationszahl eines Atoms in einer Kristallstruktur nimmt mit steigendem Druck zu - es wird ein Zustand größerer Dichte gebildet.

2. Druck-Homologenregel: Innerhalb einer Gruppe des Periodensystems nehmen die Transformationsdrücke zu höheren Atomgewichten hin ab. 
Sowie das Druck-Abstands-Paradoxon nach Kleber [36]: Während bei einer Phasenumwandlung die Koordinationszahl steigt, vergrößern sich die interatomaren Abstände im Vergleich zur Niederdruckphase.

Auch innerhalb des stabilen Bereichs einer Phase nimmt das Volumen mit zunehmendem Druck ab, da die Struktur zusammengedrückt wird.

Der treibende Faktor bei temperaturinduzierten Phasenumwandlungen ist die Zunahme der Entropie $S$. Bei steigender Temperatur nimmt die Koordinationszahl in den meisten Fällen ab und das Volumen nimmt zu.

Strukturelle Phasenumwandlungen können auf unterschiedliche Weise klassifiziert werden. Am bekanntesten sind die Einteilung anhand thermodynamischer Gesichtspunkte nach Ehrenfest [23] und die nach strukturellen Gesichtspunkten von Buerger [12]:

Diskontinuierliche Phasenumwandlungen, auch Phasenumwandlungen 1. Ordnung genannt, zeichnen sich durch eine Unstetigkeit in der ersten Ableitung der Gibbs'schen freien Energie $G$ nach dem variierten Parameter aus und es kommt am Phasenübergangspunkt zu einem plötzlichen Sprung in Entropie und Volumen. Aus Gleichung 2.42 ergeben sich folgende Beziehungen:

$$
\begin{gathered}
\left(\frac{\delta G}{\delta T}\right)_{p}=-S \\
\left(\frac{\delta G}{\delta p}\right)_{T}=V
\end{gathered}
$$

Kontinuierliche Phasenumwandlungen oder Phasenumwandlungen 2. Ordnung zeichnen sich dadurch aus, dass eine Unstetigkeit erst in der zweiten Ableitung der Gibbs'schen freien Energie $G$ nach dem variierten Parameter auftritt. Dies bedeutet, dass kein plötzlicher Entropie- und Volumensprung am Phasenübergangspunkt stattfindet. Stattdessen ändern sich Wärmekapazität $c_{p}$, Kompressibilität $\kappa$ und thermische Ausdehnung $\gamma$ der 
Substanz sprunghaft:

$$
\begin{array}{r}
\left(\frac{\delta^{2} G}{\delta T^{2}}\right)_{p}=-\left(\frac{\delta S}{\delta T}\right)_{p}=-\frac{1}{T}\left(\frac{\delta H}{\delta T}\right)_{p}=-\frac{c_{p}}{T} \\
\left(\frac{\delta^{2} G}{\delta p^{2}}\right)_{T}=\left(\frac{\delta V}{\delta p}\right)_{T}=-V \cdot \kappa \\
\left(\frac{\delta^{2} G}{\delta T \delta p}\right)=-\left(\frac{\delta S}{\delta p}\right)=V \cdot \gamma
\end{array}
$$

Buerger [12] unterscheidet rekonstruktive, dilatative, displazive und Ordnungs-UnordnungsÜbergänge, die wiederum in Substitutionsumwandlungen und rotative und distortive Umwandlungen unterteilt werden. Zu den diskontinuierlichen Phasenumwandlungen gehören folgende Phasenumwandlungstypen:

- Bei rekonstruktiven Phasenumwandlungen werden Bindungen aufgebrochen und neue geknüpft. Es kommt während der Umwandlung zur Keimbildung der neuen Phase und Einkristalle zerfallen oft in ein polykristallines Aggregat. Aufgrund der zum Aufbrechen der Bindungen nötigen hohen Aktivierungsenergie verlaufen rekonstruktive Phasenumwandlungen träge, bei einer Rückumwandlung ist eine Hysterese zu beobachten. Produkte rekonstruktiver Phasenumwandlungen können auch außerhalb ihres Stabilitätsfelds metastabil existieren.

- Bei dilatativen Phasenumwandlungen erfolgt die Umwandlung durch diffusionslose, korrelierte, kontinuierliche Verschiebung der Atome. Es findet keine Keimbildung der neuen Phase statt, es kann während des Phasenumwandlungsprozesses jedoch zu Zwillingsbildung kommen. Die Koordinationszahl ändert sich, es werden neue Bindungen geknüpft ohne bereits existierende Bindungen aufzubrechen, falls die Ausgangsphase eine niedrigere Koordinationszahl aufweist. Die Aktivierungsenergie ist im Vergleich zu rekonstruktiven Phasenumwandlungen geringer, sodass der Umwandlungsprozess weniger träge verläuft und die Existenz metastabiler Phasen nur selten und durch schnelle Abschreckung ermöglicht wird.

Auch martensitische Phasenumwandlungen gehören zu den dilatativen Transformationen. Sie zeichnen sich dadurch aus, dass die Verschiebung der Gitterbausteine nur einen Bruchteil einer Gittertranslation beträgt.

- Auch die Substitutions-Ordnungs-Unordnungs-Übergänge sind 1. Ordnung. 
Hier ändert sich die Ordnung der Atomverteilung innerhalb der Kristallstruktur. Beim Übergang geordnet verteilter Atome onterschiedlicher Atomsorten zu einer statistisch verteilten Anordnung ändert sich die Symmetrie. Hierzu müssen wie bei rekonstuktiven Phasenumwandlungen Bindungen aufgebrochen und neu geknüpft werden, was eine hohe Aktivierungsenergie erfordert. Somit erfolgen auch hier eine träge Umwandlung und die Bildung metastabiler Phasen.

$\mathrm{Zu}$ den kontinuierlichen Phasenumwandlungen gehören folgende Phasenumwandlungstypen:

- Displazive Phasenumwandlungen sind Übergänge 2. Ordnung. Koordinationspolyeder innerhalb der Kristallstruktur werden verkippt und/oder verzerrt. Dies führt zu einer Symmetrieänderung. Die Koordinationszahl ändert sich nicht und es werden keine Bindungen aufgebrochen oder neu geknüpft, sodass die Topologie der Struktur erhalten bleibt. Diffusion spielt bei diesen Vorgängen keine Rolle, was zu niedrigen Aktivierungsenergien, schnellen Abläufen der Umwandlungsprozesse und nicht abschreckbaren Phasen führt.

- Die distortiven und rotativen Phasenumwandlungen verlaufen ebenfalls kontinuierlich. So können z.B. Baugruppen wie $\mathrm{H}_{2} \mathrm{O}, \mathrm{PF}_{6}, \mathrm{BF}_{4}$ usw. in der Kristallstruktur der Hochtemperaturphase einer Verbindung frei rotieren, während sie bei tiefen Temperaturen fest ausgerichtet sind. Solche Phasenumwandlungen benötigen ebenfalls geringe Aktivierungsenergien und sind nicht abschreckbar. 


\subsection{Unter- und Obergruppen kristallographischer Raumgruppen und Transformationspfade}

Bei der Betrachtung von Phasenübergängen ist nicht nur die Raumgruppe der untersuchten Substanz von Interesse, sondern auch die Unter- und Obergruppen dieser Raumgruppe. Die Existenz eines Gruppe-Untergruppe-Zusammenhangs und damit von Orientierungs- und Symmetriebeziehungen zwischen verschiedenen Phasen einer Substanz ist für dilatative und displazive Phasenübergänge, sowie Ordnungs-UnordnungsÜbergänge bekannt, wird für rekonstruktive Phasenumwandlungen jedoch nicht erwartet [10].

\subsubsection{Unter- und Obergruppen}

Die Symmetrie einer Kristallstruktur wird mit einer Raumgruppe beschrieben. Eine Raumgruppe ist eine mathematische Gruppe, deren Gruppenelemente Symmetrieoperationen sind. Bildet eine Untermenge dieser Symmetrieoperationen wieder eine Raumgruppe, handelt es sich um eine Untergruppe der Ausgangsraumgruppe. Diese Ausgangsraumgruppe ist wiederum eine Obergruppe der betrachteten Raumgruppe. Es wird von einer maximalen Untergruppe beziehungsweise einer minimalen Obergruppe gesprochen, wenn es keine Zwischengruppe gibt. Untergruppen werden wie folgt eingeteilt [27]:

- Translationengleiche Untergruppen (Typ I): Alle Gittertranslationen der Ausgangsgruppe bleiben erhalten. Die primitiven Elementarzellen von Gruppe und Untergruppe sind gleich groß. Es ändert sich die Kristallklasse.

- Klassengleiche Untergruppen (Typ II): Klassengleiche Untergruppen gehören zur gleichen Kristallklasse wie die Ausgangsgruppe. Es werden drei Untertypen unterschieden:

Typ IIa: Die Elementarzellen von Gruppe und Untergruppe sind gleich groß, es fallen zentrierende Translationen weg.

Typ IIb: Die Elementarzelle der Untergruppe wird durch den Wegfall von Translationen vergrößert.

Typ IIc: isomorphe Untergruppen, Gruppe und Untergruppe gehören zum glei- 
chen oder dem enantiomorphen Raumgruppentyp. Die Elementarzelle vergrößert sich.

Der Abbau der Symmetrie wird mit dem Index $i$ gekennzeichnet. $i$ wird berechnet, indem die Zähligkeit der allgemeinen Lage der Ausgangsgruppe durch die Zähligkeit der allgemeinen Lage der Untergruppe geteilt wird, in Bezug auf das gleiche Elementarzellenvolumen.

\subsubsection{Transformationspfade}

Schon lange gibt es Versuche, Strukturzusammenhänge für die verschiedenen Phasen einer Substanz auch bei als rekonstruktiv angesehenen Phasenübergängen zu finden. Capillas et al [16] definieren einen Transformationspfad als eine Gruppe von Atomverschiebungen und Gitterverzerrungen, die dafür sorgen, dass das Material sich in kontinuierlicher Weise von einer Phase in die andere umwandelt.

Im Folgenden werden einige Beispiele für verschiedene Untersuchungen an Substanzen, die rekonstruktive Phasenübergänge aufweisen, gelistet:

- Shôji [67] stellte geometrische Überlegungen zu Phasenübergängen für eine Vielzahl kristalliner Substanzen an und bestimmte die Orientierungsbeziehungen, unter

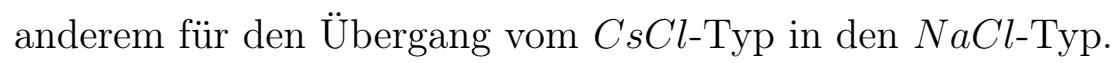

- Buerger [11] schlug einen Phasenübergang vom CsCl-Typ in den $N a C l$-Typ durch Dilatation entlang einer [111]-Richtung vor.

- Corll [19] betrachtete die per Ultraschall gemessenen elastischen Konstanten von $C d S$ unter Druck und entwickelte ein Phasenübergangsmodell, das auf kontinuierlicher Verformung durch mikroskopische Schermechanismen basiert.

- Hyde et al. [33] schlugen zwei energetisch äquivalente Modelle für den Phasen-

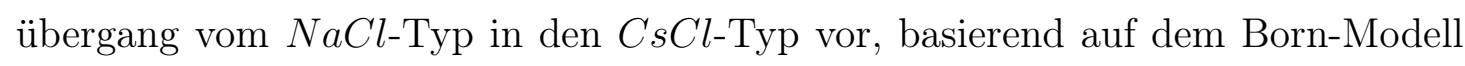
der Ionenkristalle, mit welchem die minimale Energie zwischen dem anziehenden elektrostatischen Potential des Ionengitters und Abstoßungskräften gleich geladener Ionen berechnet werden und somit der ideale Atomabstand bestimmt werden kann (Born-Landé-Gleichung). 
- Christy [18] betrachtete mögliche Transformationspfade für $M X$ - und $M X_{2}$-Verbindungen sowie für $\mathrm{CaCO}_{3}$ basierend auf strukturellen Ähnlichkeiten und Gitterschwingungsmoden durch molekulardynamische Simulationen. Er schlug vor, nach strukturellen Gemeinsamkeiten der Phasen vor und nach dem Phasenübergang sowie passenden Gitterschwingungen, die diese erhalten, zu suchen. Dies erlaubt, den kürzesten Transformationspfad zu finden und die Symmetrie einer Zwischenphase zu bestimmen.

- Dmitriev et al. [21], [22] definierten einen Ordnungsparameter der Landau-Theorie für rekonstruktive Phasenübergänge und demonstrierten diesen an zahlreichen Beispielen von Phasenübergängen, z.B. in Elementkristallen. Sie gehen allerdings davon aus, dass keine Gruppe-Untergruppe-Beziehungen zwischen den Phasen existieren.

- Tolbert et al. [82] untersuchten den Phasenübergang vom Wurtzit-Typ in den $\mathrm{NaCl}$-Typ in $\mathrm{CdSe-Nanokristallen} \mathrm{unter} \mathrm{Hochdruck} \mathrm{in} \mathrm{einer} \mathrm{Diamantstempelzelle.}$ Aus den im Experiment gewonnenen thermodynamischen Daten und der berechneten Oberflächenenergie der Nanokristalle leiteten sie mögliche Pfade der Atome während des Phasenübergangs her.

- Sharma et al. [64] nutzten Schockwellen um einen Phasenübergang vom WurtzitTyp in den $N a C l$-Typ in $C d S$ herbeizuführen und und hielten eine flächenzentrierten orthorhombischen Typ als metastabile Phase zwischen den beiden Endzuständen für wahrscheinlich. Knudson et al. [37] kamen mit der gleichen Methode auf einen flächenzentrierten tetragonalen Typ als wahrscheinliche metastabile Phase.

- Shimojo et al. [65], [66] simulierten den Phasenübergang vom Zinkblende- zum

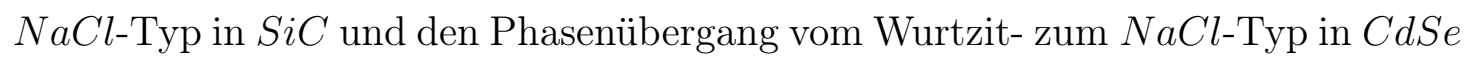
mit Hilfe molekulardynamischer Methoden.

- Miao et al. [48] berechneten über Pseudopotentiale die Enthalpiebarrieren für die Phasenübergang verschiedener SiC-Modifiaktionen in den $\mathrm{NaCl}$-Typ und fanden die niedrigste Enthalpiebarriere für den Zinkblende-Typ als Ausgangsmodifikation.

- Wickham et al. [89] untersuchten $C d S e$-Nanokristalle mit Röntgendiffraktometrie während wiederholter Phasenübergänge vom Wurtzit-Typ in den $\mathrm{NaCl}$-Typ und 
zurück und betrachteten anschließend die dadurch hervorgerufenen Formänderungen der Nanokristalle und Stapelfehler im Gitter um mögliche Phasenübergangsmechanismen einzugrenzen.

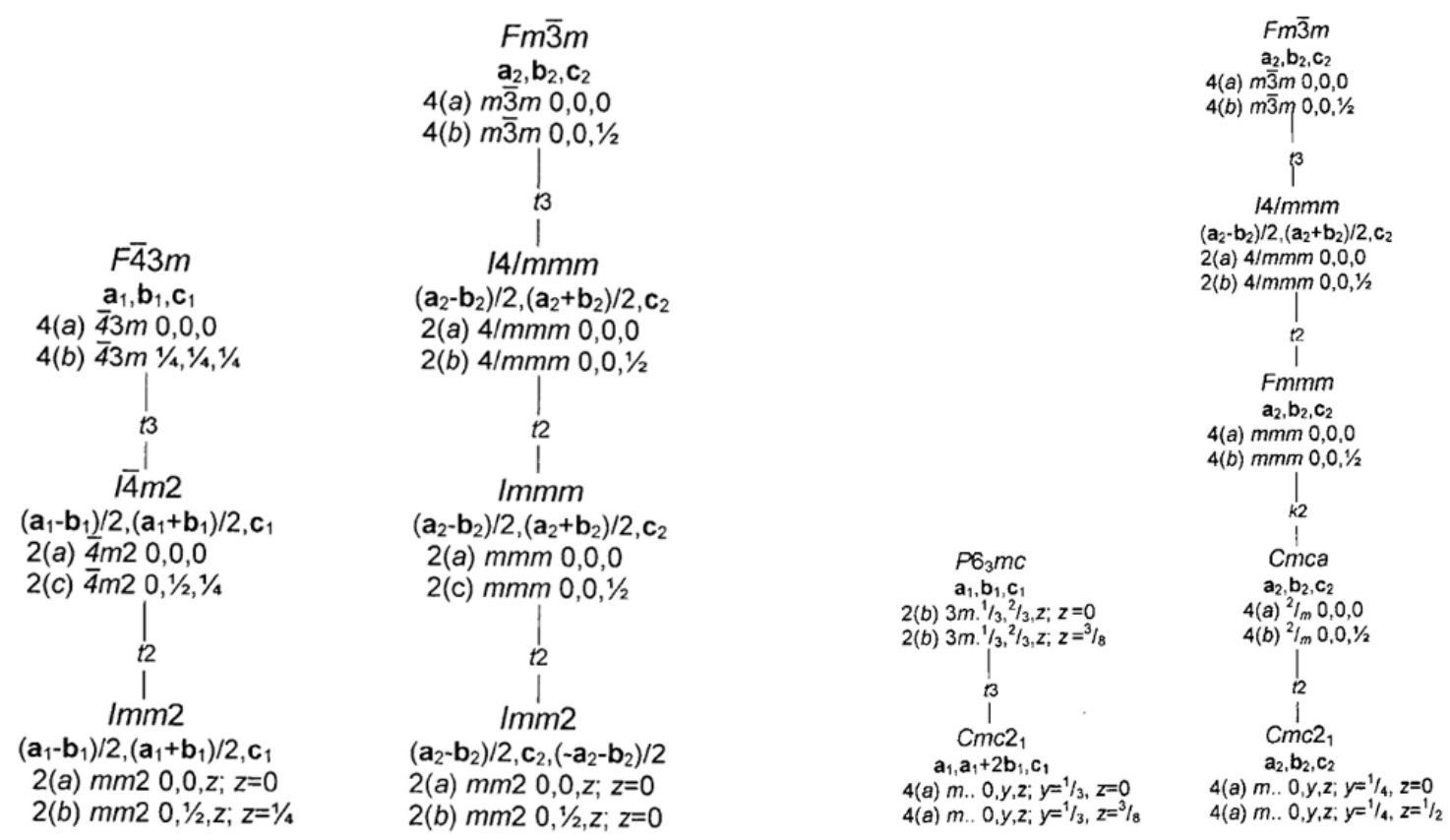

Abbildung 2.9: Beispiele für Transformationspfade über eine gemeinsame Untergruppe: Links für die Phasenumwandlung vom Zinkblende-Typ $(F \overline{4} 3 m)$ in den $N a C l$-Typ $(F m \overline{3} m)$ über die gemeinsame Untergruppe Imm2. Rechts für die Phasenumwandlung vom Wurtzit-Typ $\left(P 6_{3} m c\right)$ in den $\mathrm{NaCl}$-Typ $(F m \overline{3} m)$ über die gemeinsame Untergruppe $C m c 2_{1}$. Angegeben sind jeweils die Raumgruppen, die Gittervektoren der Elementarzelle bezogen auf die Ausgangsraumgruppe sowie die Atompositionen. $t i$ und $k i$ kennzeichnen translationenbzw. klassengleiche Übergänge. $i$ ist der Index der Untergruppe. (Quelle: Sowa, [68], [69])

Seit ca. 20 Jahren wird versucht, Transformationspfade für druckinduzierte Phasenübergänge zu finden, indem man nach einer gemeinsamen Untergruppe der Raumgruppen der Phasen vor und nach dem Phasenübergang sucht, die es ermöglicht, einen Strukturtyp in den anderen zu überführen. Hierfür erfolgt ein schrittweiser Abbau der Symmetrie von Ausgangs- und Endphase mit Verschiebung der Elementarzellenkoordinaten und/oder Änderung der Elementarzellengröße bis zu einer gemeinsamen Untergruppe (siehe Abbildung 2.9). Theoretisch sind zahlreiche Transformationspfade für einen gegebenen Phasenübergang möglich, praktisch spielen auch thermodynamische Aspekte eine Rolle bei der Einschätzung, ob ein gefundener Transformationspfad realistisch ist. Ein energetisch günstiger Transformationspfad zeichnet sich dadurch aus, dass während des Übergangs keine oder nur wenige atomare Bindungen aufgebrochen werden müssen und die bei der Verschiebung der Atome innerhalb der Elementarzelle zurückgelegten 
Strecken möglichst kurz sind. Die im folgenden aufgeführten Arbeiten sind nach den jeweiligen für die vorliegende Arbeit relevanten Phasenübergängen geordnet.

Übergang vom Zinkblende- zum $\mathrm{NaCl-Typ:}$

- Sowa [68] fand mit Hilfe von Kugelpackungsüberlegungen einen Phasenübergang über die gemeinsame Untergruppe Imm2. Die daraus resultierenden Orientierungsbeziehungen sind $[001]_{Z n S} \|[\overline{1} \overline{1} 0]_{N a C l}$ und $[110]_{Z n S} \|[001]_{N a C l}$.

- Blanco et al. [7] untersuchten den Phasenübergang über die gemeinsame Untergruppe $R 3 m$ mit Hilfe von berechneten Potentialflächen am Beispiel $\mathrm{ZnO}$ und bestimmten die atomaren Abstände im Verlauf der Transformation. Direkte Angaben über Orientierungsbeziehungen zwischen den Phasen wurden nicht gemacht, aber aus den Daten lässt sich ableiten, dass die Hauptachsen der Elementarzelle nach dem Phasenübergang erhalten bleiben: $\langle 100\rangle_{Z n S} \|\langle 100\rangle_{\mathrm{NaCl}}$.

- Catti et al [17] führten Dichtefunktionsberechnungen mit Linearkombinationen von Atomorbitalen an $\mathrm{SiC}$ durch und fanden als energetisch günstigsten Phasenübergang einen Übergang mit der gemeinsamen orthorhombischen Untergruppe Pmm2.

- Ebenfalls von Sowa [70] stammt die auf Kugelpackungsüberlegungen basierende Herleitung eines Phasenübergangs über die gemeinsame Untergruppe $P 3_{2}$. Der Mechanismus entspricht einer Drehung der Elementarzelle um $180^{\circ}$ um die Flächennormale der (111)-Netzebene. Die daraus resultierende Orientierungsbeziehung ist $[111]_{Z n S} \|[111]_{N a C l}$.

- Hatch et al. [30] untersuchten mit gruppentheoretischer Analyse und Elektronenstrukturberechnungen mögliche Transformationspfade in $\mathrm{SiC}$ für den 'Bilayer Sliding Mechanism', welcher aus der Verschiebung der (111)-Netzebenen gegeneinander besteht, so dass die Koordinationszahl der Atome von 4 auf 6 erhöht wird. Sie fanden acht Transformationspfade mit ähnlich niedriger Aktivierungsenergie, welche alle ohne das Aufbrechen von Bindungen auskommen und sich nur durch die Reihenfolge der Richtung der Verschiebungen unterscheiden. Aus diesen unterschiedlichen Verschiebungsreihenfolgen resultieren unterschiedliche gemeinsame 
Untergruppen für den Phasenübergang, als besonders vorteilhaft gilt der Phasenübergangsmechanismus über die Untergruppe $P 3_{2}$, da er als einziger der gefundenen Phasenübergänge keine Scherspannung ausübt. Dies ist der Übergang, der von Sowa [70] mit Hilfe der Kugelpackungsüberlegungen hergeleitet wurde. Ebenso wurde der von Sowa [68] hergeleitete Phasenübergang über Imm2 gefunden. Allen acht Transformationspfaden gemein ist die Orientierungsbeziehung $(111)_{Z n S} \|(111)_{N a C l}$.

- Capillas et al. [16] untersuchten den Übergang vom Zinkblende- zum $\mathrm{NaCl-Typ} \mathrm{an}$ SiC mit Hilfe gruppentheoretischer Methoden und Atomverschiebungen. Für den Übergang vom Zinkblende- zum $\mathrm{NaCl}$-Typ wurden sieben Transformationspfade mit maximaler Symmetrie gefunden, zu denen auch die beiden von Sowa [68], [70] hergeleiteten Transformationspfade über $I m m 2$ und $P 3_{2}$ gehören.

Übergang vom Wurtzit- zum NaCl-Typ:

- Sowa [69] fand mit Hilfe von Kugelpackungsüberlegungen einen Phasenübergang über die gemeinsame Untergruppe $C m c 2_{1}$. Die daraus resultierenden Orientierungsbeziehungen sind $[100]_{W z}\left\|[100]_{N a C l},[120]_{W z}\right\|[010]_{N a C l}$ und $[001]_{W z} \|[001]_{N a C l}$.

- Ebenfalls von Sowa [71] stammt die auf Kugelpackungsüberlegungen basierende Beschreibung eines solchen Phasenübergangs [66] über die gemeinsame Untergruppe $P n a 2_{1}$. Daraus resultieren die Orientierungsbeziehungen $[210]_{W z} \|[110]_{N a C l}$, $[010]_{W z} \|[\overline{1} 10]_{N a C l}$ und $[001]_{W z} \|[001]_{N a C l}$.

- Stokes et al. [80] simulierten mit gruppentheoretischer Analyse und Elektronenstrukturberechnungen den 'Bilayer Sliding Mechanism' für den Phasenübergang vom Wurtzit- zum $\mathrm{NaCl}$-Typ und erhielten fünf Phasenübergangsmodelle, die ohne das Aufbrechen von Bindungen auskommen. Die Transformationspfade basieren auf der Verschiebung der hexagonalen (010)-Netzebenen entlang der hexagonalen $\langle 100\rangle$-Netzebenen, was die Koordinationszahl der Atome von 4 auf 6 erhöht, und unterscheiden sich sich nur durch die Reihenfolge der Richtung der Verschiebungen. Allen fünf Transformationspfaden gemein ist die Orientierungsbeziehung $[001]_{W z} \|[\overline{001}]_{N a C l}$. Zwei dieser fünf Transformationspfade (gemeinsame Untergruppen $C m c 2_{1}$ und $\left.P n a 2_{1}\right)$ entsprechen den von Sowa in ([69] und [71]) hergeleiteten Pfaden. 
- Capillas et al. [16] untersuchten den Phasenübergang vom Wurtzit- zum NaCl-Typ an GaN mit Hilfe gruppentheoretischer Methoden und Atomverschiebungen. Für den Übergang vom Wurtzit- zum NaCl-Typ wurden acht Transformationspfade mit maximaler Symmetrie gefunden, zu ihnen gehören die von Sowa ([69], [71]) hergeleiteten Pfade über die gemeinsame Untergruppen $C m c 2_{1}$ und $P n a 2_{1}$, die übrigen erhaltenen Pfade decken sich allerdings nicht mit denen von Stokes et al. $[80]$.

Keine der erwähnten Arbeiten zu Orientierungsbeziehungen zwischen zwei Phasen basiert auf strukturellen experimentellen Untersuchungen.

\subsubsection{Achsen- und Koordinatentransformationen}

Um die Gittervektoren $\vec{a}, \vec{b}, \vec{c}$ der Elementarzelle der Ausgangsphase in die Gittervektoren $\vec{a}^{\prime}, \vec{b}^{\prime}, \vec{c}^{\prime}$ der Elementarzelle der Endphase zu transformieren wird eine Achsentransformation mit Hilfe einer Matrix durchgeführt [27]. Deren Koeffizienten ergeben sich aus den einzelnen Komponenten der Gittervektoren:

$$
\begin{aligned}
& \vec{a}^{\prime}=p_{11} \vec{a}+p_{21} \vec{b}+p_{31} \vec{c} \\
& \vec{b}^{\prime}=p_{12} \vec{a}+p_{22} \vec{b}+p_{32} \vec{c} \\
& \vec{c}^{\prime}=p_{13} \vec{a}+p_{23} \vec{b}+p_{33} \vec{c}
\end{aligned}
$$

Somit lassen sich die Gittervektoren folgendermaßen transformieren:

$$
\left(\vec{a}^{\prime}, \vec{b}^{\prime}, \vec{c}^{\prime}\right)=(\vec{a}, \vec{b}, \vec{c}) \cdot\left(\begin{array}{lll}
p_{11} & p_{12} & p_{13} \\
p_{21} & p_{22} & p_{23} \\
p_{31} & p_{32} & p_{33}
\end{array}\right)=(\vec{a}, \vec{b}, \vec{c}) \cdot P
$$

Ebenso gilt umgekehrt für die Rücktransformation:

$$
\begin{aligned}
& \vec{a}=q_{11} \vec{a}^{\prime}+q_{21} \vec{b}^{\prime}+q_{31} \vec{c}^{\prime} \\
& \vec{b}=q_{12} \vec{a}^{\prime}+q_{22} \vec{b}^{\prime}+q_{32} \vec{c}^{\prime} \\
& \vec{c}=q_{13} \vec{a}^{\prime}+q_{23} \vec{b}^{\prime}+q_{33} \vec{c}^{\prime}
\end{aligned}
$$


mit der Matrix

$$
(\vec{a}, \vec{b}, \vec{c})=\left(\vec{a}^{\prime}, \vec{b}^{\prime}, \vec{c}^{\prime}\right) \cdot\left(\begin{array}{lll}
q_{11} & q_{12} & q_{13} \\
q_{21} & q_{22} & q_{23} \\
q_{31} & q_{32} & q_{33}
\end{array}\right)=\left(\vec{a}^{\prime}, \vec{b}^{\prime}, \vec{c}^{\prime}\right) \cdot Q
$$

Die Matrizen $P$ und $Q$ sind invers zueinander, d. h. $P=Q^{-1}$ und umgekehrt.

Um von der Ausgangsphase zur Endphase zu kommen, sind bei einem Transformationspfad über eine gemeinsame Untergruppe eine endliche Anzahl $n$ von Zwischenschritten nötig. Für jeden Zwischenschritt wird eine eigene Transformationsmatrix erstellt. Diese werden dann zur Transformationsmatrix für den gesamten Pfad folgendermaßen verknüpft:

$$
\begin{gathered}
Q_{\text {gesamt }}=Q_{n} \circ Q_{n-1} \circ \ldots \circ Q_{1} \\
P_{\text {gesamt }}=P_{1} \circ \ldots \circ P_{n-1} \circ P_{n}
\end{gathered}
$$

Für die Transformation der Millerschen Indizes und damit für die Berechnung der Orientierungsbeziehungen zwischen den Phasen gilt:

$$
\begin{aligned}
& \left(h^{\prime}, k^{\prime}, l^{\prime}\right)=(h, k, l) \cdot P \\
& (h, k, l)=\left(h^{\prime}, k^{\prime}, l^{\prime}\right) \cdot Q
\end{aligned}
$$

Gelegentlich ändert sich bei einem Übergang von einer Gruppe zu einer Untergruppe auch der Ursprung der Gittervektoren. Die Verschiebung des Ursprungs wird durch die Vektoren $\vec{p}$ bzw. $\vec{q}$ beschrieben:

$$
\begin{array}{r}
\vec{p}=p_{1} \vec{a}+p_{2} \vec{b}+p_{3} \vec{c} \\
\vec{q}=q_{1} \vec{a}^{\prime}+q_{2} \vec{b}^{\prime}+q_{3} \vec{c}^{\prime}
\end{array}
$$


Die Koordinaten $x, y, z$ eines beliebigen Gitterpunkts werden damit folgendermaßen transformiert:

$$
\begin{aligned}
& \left(\begin{array}{l}
x^{\prime} \\
y^{\prime} \\
z^{\prime}
\end{array}\right)=Q \cdot\left(\begin{array}{l}
x \\
y \\
z
\end{array}\right)+q \\
& \left(\begin{array}{l}
x \\
y \\
z
\end{array}\right)=P \cdot\left(\begin{array}{l}
x^{\prime} \\
y^{\prime} \\
z^{\prime}
\end{array}\right)+p
\end{aligned}
$$




\section{Die Probensubstanzen und ihre kristallographischen Daten}

Das Probenmaterial für die Experimente mit Indiumarsenid (InAs), Cadmiumsulfid $(C d S)$ und Cadmiumselenid $(C d S e)$ wurde bei der MaTecK Material, Technologie \& Kristalle GmbH erworben, das Probenmaterial für die Experimente mit Kupferindiumdiselenid (CuInSe) wurde freundlicherweise von Prof. Dr. K. Bente (Universität Leipzig) zur Verfügung gestellt. Die Millerit (NiS)-Kristalle aus Wissen/Sieg, Deutschland, stammen aus der mineralogischen Sammlung der TU Berlin.

\subsection{Indiumarsenid $(\operatorname{In} A s)$}
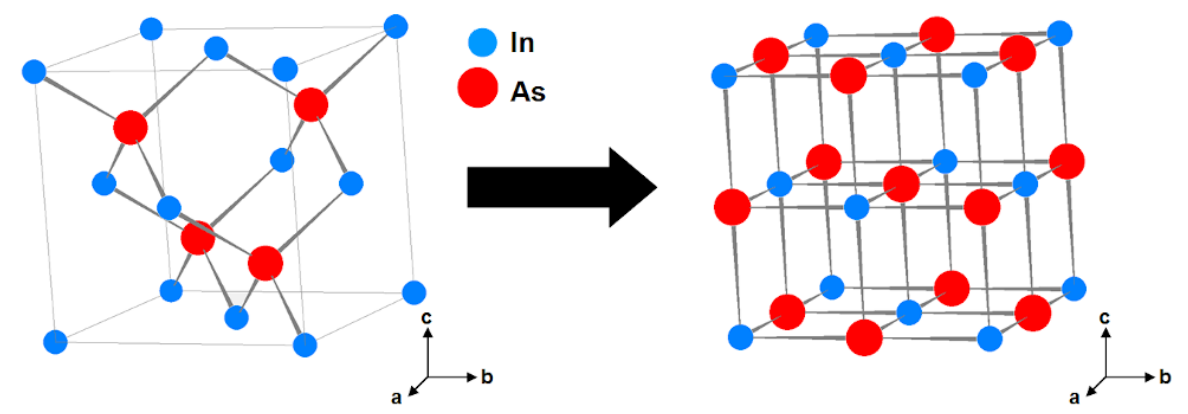

Abbildung 3.1: Kristallstrukturen des Zinkblende-Typs (links) und des NaCl-Typs (rechts). Die schwarzen Linien in der linken Abbildung zeigen die Grenzen der Elementarzelle.

Indiumarsenid ist ein $I I I-V$-Halbleitermaterial, das unter anderem für Photodioden in Infrarotdetektoren und Solarzellen verwendet wird. Unter Standardbedingungen kristallisiert InAs im Zinkblende-Typ mit der Raumgruppe $F \overline{4} 3 m$. Die Kantenlänge der Elementarzelle beträgt $\mathrm{a}=6,058 \AA[24]$. Die Indium-Kationen besetzen die Punktlage 4a 
0,0,0 , während die Arsen-Anionen die Punktlage 4c $\frac{1}{4}, \frac{1}{4}, \frac{1}{4}$ besetzen. Die Koordinationszahl beträgt 4. Im Zinkblende-Typ bilden die Kationen und Anionen in $\langle 111\rangle_{Z n S^{-}}$ Richtung Doppelschichten, deren Verschiebung gegeneinander während des Phasenübergangs für mögliche Transformationspfade interessant ist (siehe Hatch et al. [30]). Bei einem Druck von 7 GPa ([85], [2]) wandelt sich die Kristallstruktur träge zum NaCl-Typ mit der Raumgruppe $F m \overline{3} m$ um, was mit einer Verringerung des Elementarzellenvolumens um 17\% einhergeht (siehe Abbildung 3.1). Die Kantenlänge der Elementarzelle beträgt $\mathrm{a}=5.501 \AA$ bei einem Druck von 8.46 GPa [85]. Die Indium-Kationen befinden sich auf der Punktlage 4a 0, 0, 0, während die Arsen-Anionen nun die Punktlage 4b $\frac{1}{2}, \frac{1}{2}, \frac{1}{2}$ einnehmen. Die Koordinationszahl der Atome steigt auf 6.

\subsection{Cadmiumsulfid $(C d S)$}
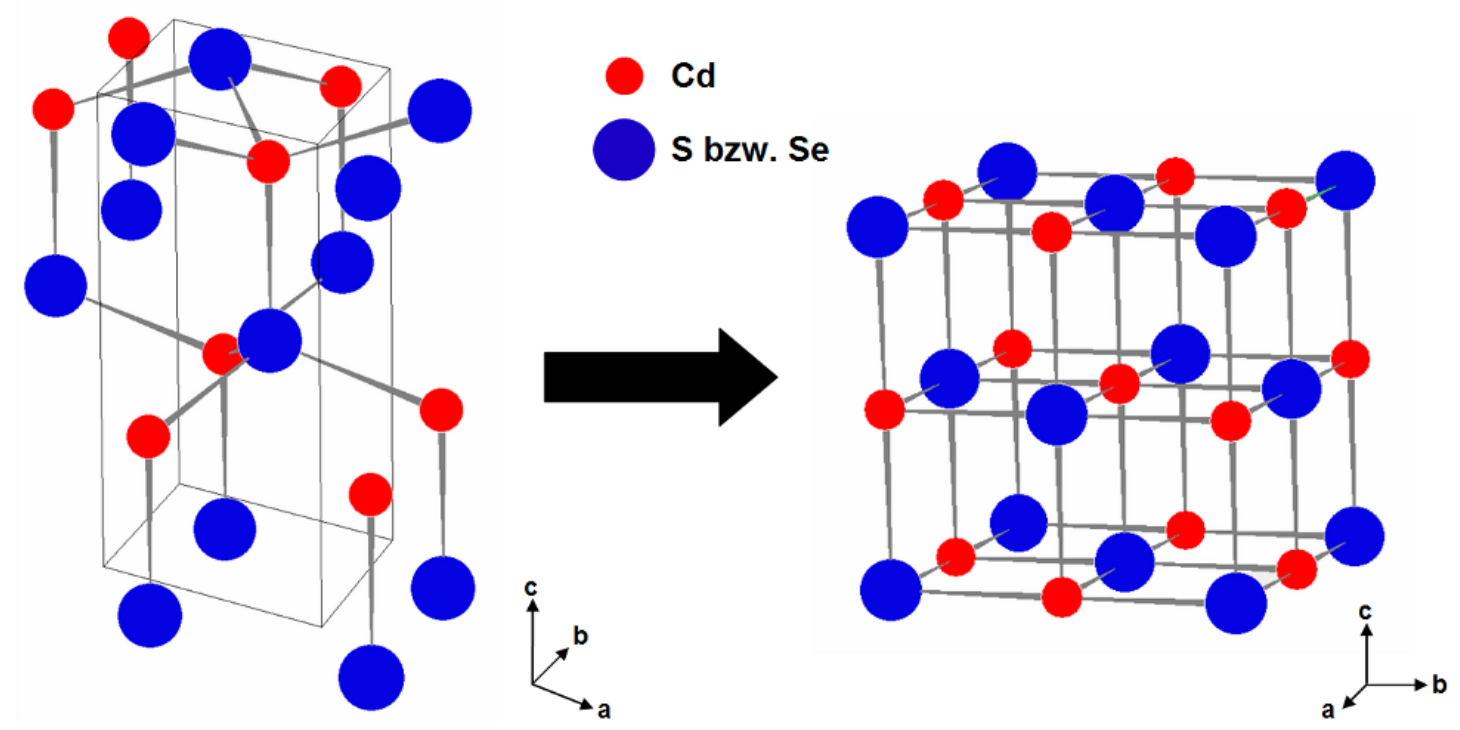

Abbildung 3.2: Kristallstrukturen des Wurtzit-Typs (links) und des NaCl-Typs (rechts). Die schwarzen Linien in der linken Abbildung zeigen die Grenzen der Elementarzelle.

Cadmiumsulfid ist ein in zwei Modifikationen in der Natur vorkommendes Material, dessen Halbleitereigenschaften in der Industrie zum Bau von Fotowiderständen und Belichtungsmessern genutzt werden. Ebenso ist es als Pigment in der Farbe Cadmiumgelb zu finden. Die im Rahmen der vorliegenden Arbeit untersuchte thermodynamisch stabile hexagonale Modifikation Greenockit kristallisiert im Wurtzit-Typ mit der Raumgruppe $\mathrm{P}_{3} m c$ und einer Koordinationszahl von 4. Die Gitterparameter betragen 
$\mathrm{a}=4.138 \AA$ und $\mathrm{c}=6.718 \AA[32]$. Sowohl die Cadmium-Kationen als auch die SchwefelAnionen besetzen die Punktlage $2 \mathrm{~b} \frac{1}{3}, \frac{2}{3}, z$ mit $z=0$ für die Cadmium-Kationen und $z=0.37715$ für die Schwefel-Anionen [78]. Bei einem Druck zwischen 2 und 3 GPa ([6],[35],[53],[60],[61],[62],[72]) erfolgt eine Umwandlung der Kristallstruktur in den NaClTyp (Raumgruppe $F m \overline{3} m$ ) mit einem Anstieg der Koordinationszahl von 4 auf 6 (siehe Abbildung 3.2). Die Cadmium-Kationen befinden sich auf der Punktlage 4a 0, 0, 0, während die Schwefel-Anionen die Punktlage 4b $\frac{1}{2}, \frac{1}{2}, \frac{1}{2}$ einnehmen. Der Gitterparameter des NaCl-Typs beträgt a=5.521 Å bei einem Druck von $4.3 \mathrm{GPa}$ [41].

\subsection{Cadmiumselenid $(C d S e)$}

Cadmiumselenid tritt in der Natur als das Mineral Cadmoselit auf. Trotz seiner Halbleitereigenschaften findet die Substanz in der Industrie aufgrund ihrer hohen Giftigkeit nur selten Verwendung. Unter Standardbedingungen kristallisiert CdSe im Wurtzit-Typ mit der Raumgruppe $P 6{ }_{3} m c$, einer Koordinationszahl von 4 und den Gitterparametern $\mathrm{a}=4.302 \AA$ und $\mathrm{c}=7.014 \AA$ [32]. Cadmium-Kationen und Schwefel-Anionen besetzen jeweils die Punktlage 2b $\frac{1}{3}, \frac{2}{3}, z$ mit $z=0$ für die Cadmium-Kationen und $z=0.37596$ für die Selen-Anionen [79]. Bei einem Druck von 2.5 bis 3.5 GPa ([31], [35],[46],[49],[52],[60], [73],[93]) erfolgt eine Umwandlung in den NaCl-Typ mit einem Anstieg der Koordinationszahl von 4 auf 6 und einem Gitterparameter von $\mathrm{a}=5.5445 \AA$ bei $4.8 \mathrm{GPa}$ [40] (siehe Abbildung 3.2). Die Cadmium-Kationen befinden sich auf der Punktlage 4a 0, 0, 0, während die Selen-Anionen die Punktlage $4 b \frac{1}{2}, \frac{1}{2}, \frac{1}{2}$ einnehmen.

\subsection{Kupferindiumdiselenid ( $\left.\mathrm{CuInSe} \mathrm{I}_{2}\right)$}

Kupferindiumdiselenid gehört zu den $I-I I I-V I_{2}$-Halbleitern und findet aufgrund seines hohen Lichtabsorptionsgrads in Dünnschichtsolarzellen Verwendung. CuInSe $e_{2}$ kristallisiert unter Standardbedingungen im tetragonalen Chalcopyrit-Typ mit der Raumgruppe $I \overline{4} 2 d$. Dieser stellt eine Überstruktur des kubischen Zinkblende-Typs dar. Im Fall des $\mathrm{CuInSe_{2 }}$ sorgt das Vorhandensein zweier unterschiedlicher Kationen für eine Verdoppelung der c-Achse der Elementarzelle im Vergleich zum Zinkblende-Typ und somit zu einer Reduktion der Symmetrie. Die Kupfer-Kationen besetzen die Punktlage 

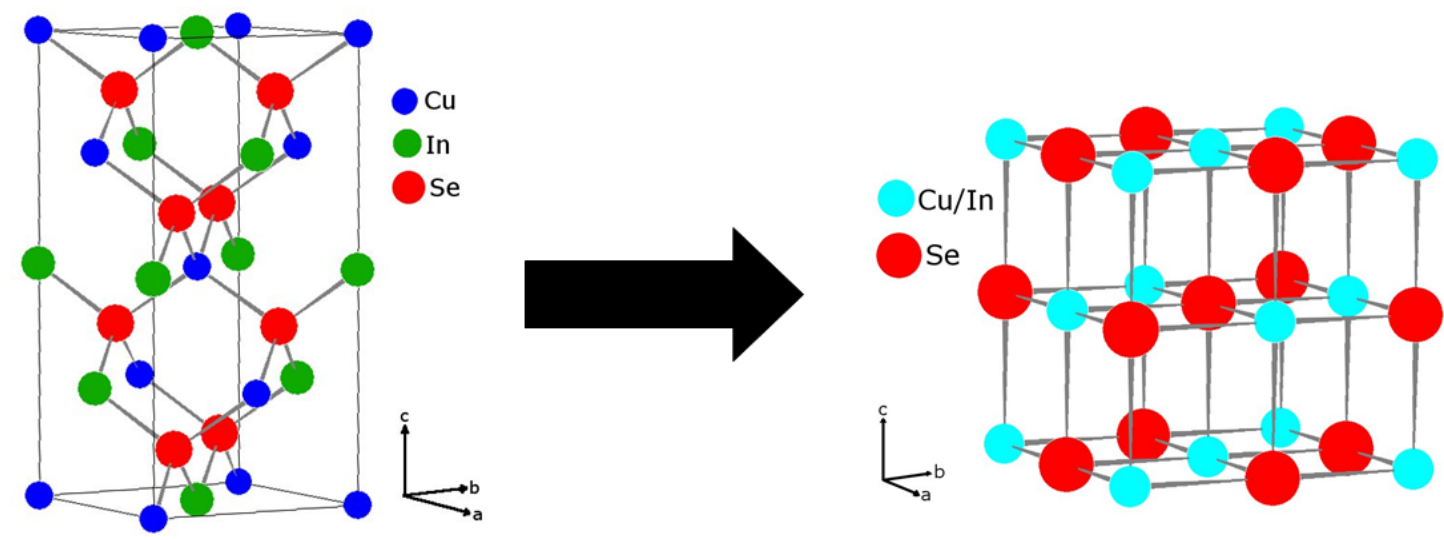

Abbildung 3.3: Kristallstrukturen des Chalcopyrit-Typs (links) und des NaCl-Typs (rechts). Die schwarzen Linien in der linken Abbildung zeigen die Grenzen der Elementarzelle.

4a mit den Koordinaten 0,0,0. Die Indium-Anionen liegen auf der Wyckoff-Position 4b $0,0, \frac{1}{2}$, während die Selen-Anionen die Punktlage 8b mit den Koordinaten $x, \frac{1}{4}, \frac{1}{8}$ mit $x=$ 0.2605 besetzen. Die Gitterparameter bei Standardbedingungen betragen $a=5.7820(2)$ $\AA$ und $\mathrm{c}=11.6173(4) \AA$, jedoch sind Schwankungen der Parameter durch nichtstöchiometrische Elementzusammensetzung bekannt [59]. Bei einem Druck von 6 bis $8 \mathrm{GPa}$ ([81],[25],[84],[92],[8]) erfolgt eine Umwandlung in den NaCl-Typ mit einer angenommenen zufälligen Verteilung der Kationen und einer Reduktion des Elementarzellenvolumens um 17\% (siehe Abbildung 3.3). Die Koordinationszahl steigt von 4 nach 6. Der Gitterparameter beträgt $\mathrm{a}=5.35 \AA[81]$. Bei Druckentlastung beginnt die Rückumwandlung bei $4.2 \mathrm{GPa}$ und das entlastete Material zeigt den Zinkblende-Typ [81].

Eine Elementanalyse des verwendeten Probenmaterials mit der Elektronenstrahl-Mikrosonde ergab eine Zusammensetzung aus 24.98(8) mol\% Kupfer, 26.44(8) mol\% Indium und 48.58(9) mol\% Selen, was eine reale chemische Formel von $C u_{0.999} I n_{1.058} S e_{1.943}$ ergibt. Dies steht in Einklang mit dem Ergebnis von Experimenten zum Ziehen von

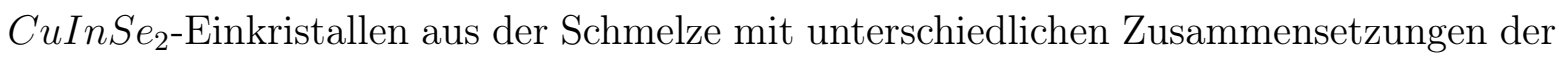
Ausgangsschmelze, bei denen sich selbst bei einem Kupferüberschuss in der Ausgangsschmelze ein Indiumüberschuss im gezogenen Einkristall ergab und sich das KationenAnionenverhältnis nie perfekt stöchiometrisch zeigte [50]. 


\subsection{Nickelsulfid $(N i S)$}

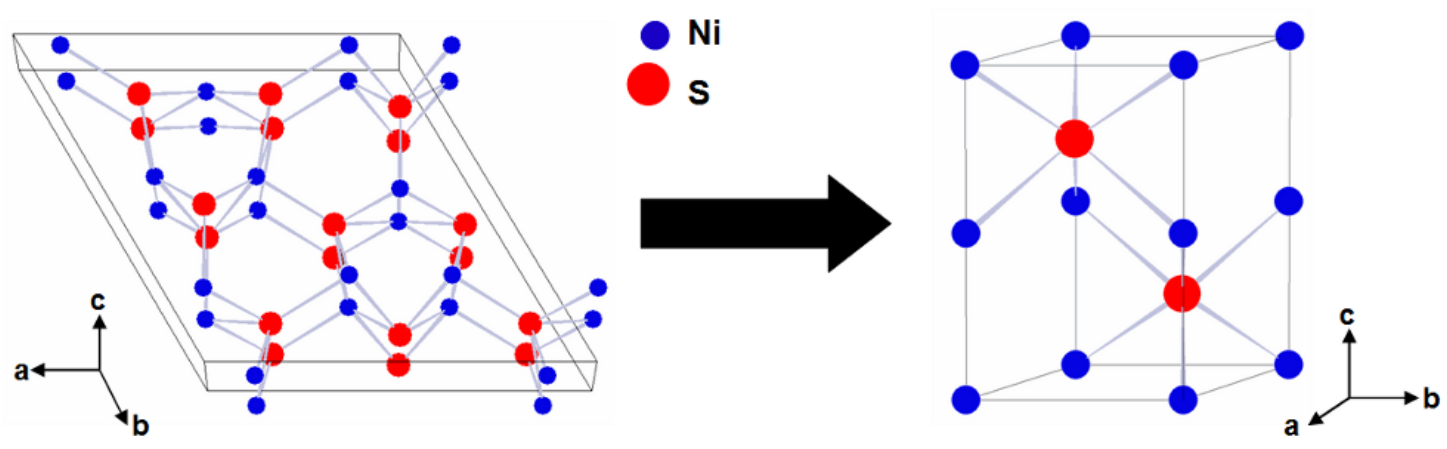

Abbildung 3.4: Kristallstrukturen des Millerit-Typs (links) und des Nickelarsenid-Typs (rechts). Die schwarzen Linien zeigen jeweils die Grenzen der Elementarzelle.

Nickel(mono)sulfid existiert in der Natur als das Mineral Millerit und kristallisiert unter Standardbedingungen im nach diesem Mineral benannten trigonalen Millerit-Typ mit der Raumgruppe $R 3 m$ und den Gitterparametern $a=9.6071 \AA$ und $c=3.1434 \AA[26]$. Die Koordinationszahl beträgt 5. Die Nickelatome sind pyramidal von Schwefelatomen umgeben. Sowohl Kationen als auch Anionen besetzen die Punktlage 9b auf den Koordinaten $x,-x, z$ mit $x=0.91220$ und $z=0.47550$ für die Ni-Atome und $x=0.11220$ und $z=0$ für die S-Atome [26]. Bei einer Temperatur von ca. $379^{\circ} \mathrm{C}$ und Standarddruck erfolgt eine Umwandlung in den hexagonalen Nickelarsenid-Typ [38] mit der Raumgruppe $P 6_{3} / m m c$ und den Gitterparametern a =3.4395 $\AA$ und $\mathrm{c}=5.3514 \AA$ (siehe Abbildung 3.4) [83]. Die Kationen besetzen die Punktlage 2a 0, 0, 0; die Anionen besetzen die Punktlage 2c $\frac{1}{3}, \frac{2}{3}, \frac{1}{4}$. Die Koordinationszahl beträgt 6, die Anordnung der Schwefelatome um die Nickelatome ist oktaedrisch. 


\title{
4 Probenpräparation
}

\subsection{Hochdruckexperimente}

\begin{abstract}
Alle Hochdruckexperimente wurden mit Diamantstempelzellen (DACs) durchgeführt. Eine solche Zelle besteht aus zwei Diamanten mit parallel angeordneten Culets (die glatt polierte Fläche, die Ober- und Unterseite der Druckkammer bildet), auf die durch ein System von Schrauben Druck ausgeübt werden kann. Alle für die vorliegenden Experimente verwendeten Diamantstempelzellen wurden von $\mathrm{H}$. Ahsbahs in Marburg gebaut (siehe Abbildung 4.1) und haben einen Öffnungswinkel von 90 (Ausnahme: 'Bunte Zelle', deren Öffnungswinkel auf einer Seite nur $60^{\circ}$ beträgt). Geschlossen werden die Diamantstempelzellen mit einem auf die jeweilige Zelle angepassten Getriebe.
\end{abstract}

1. Runde Vierschraubenzelle nach modifiziertem Mao-Bell-Typ [44] ('Bunte Zelle') mit Diamantsitz aus Beryllium (siehe Abbildung 4.2a).

2. Kleine Vierschraubenzelle nach modifiziertem Merrill-Bassett-Typ [47] mit Diamantsitz aus Beryllium (siehe Abbildung 4.2a).

3. 'Quadratische' [3] 3Dia-Zelle mit vier Schrauben [4]. Diese Zelle weist einen aus zwei Teilen bestehenden Diamantstempel auf. Aufgrund der beinahe halbkugelförmigen Form der Diamanten ist keine Absorptionskorrektur notwendig (siehe Abbildung 4.2b).

4. LAS1-Zelle [74]. Diese ist eine Weiterentwicklung der 3Dia-Zelle mit zwei einteiligen, durch Lasertechnik zugeschnittenen Diamantstempeln (siehe Abbildung $4.2 \mathrm{c})$. 


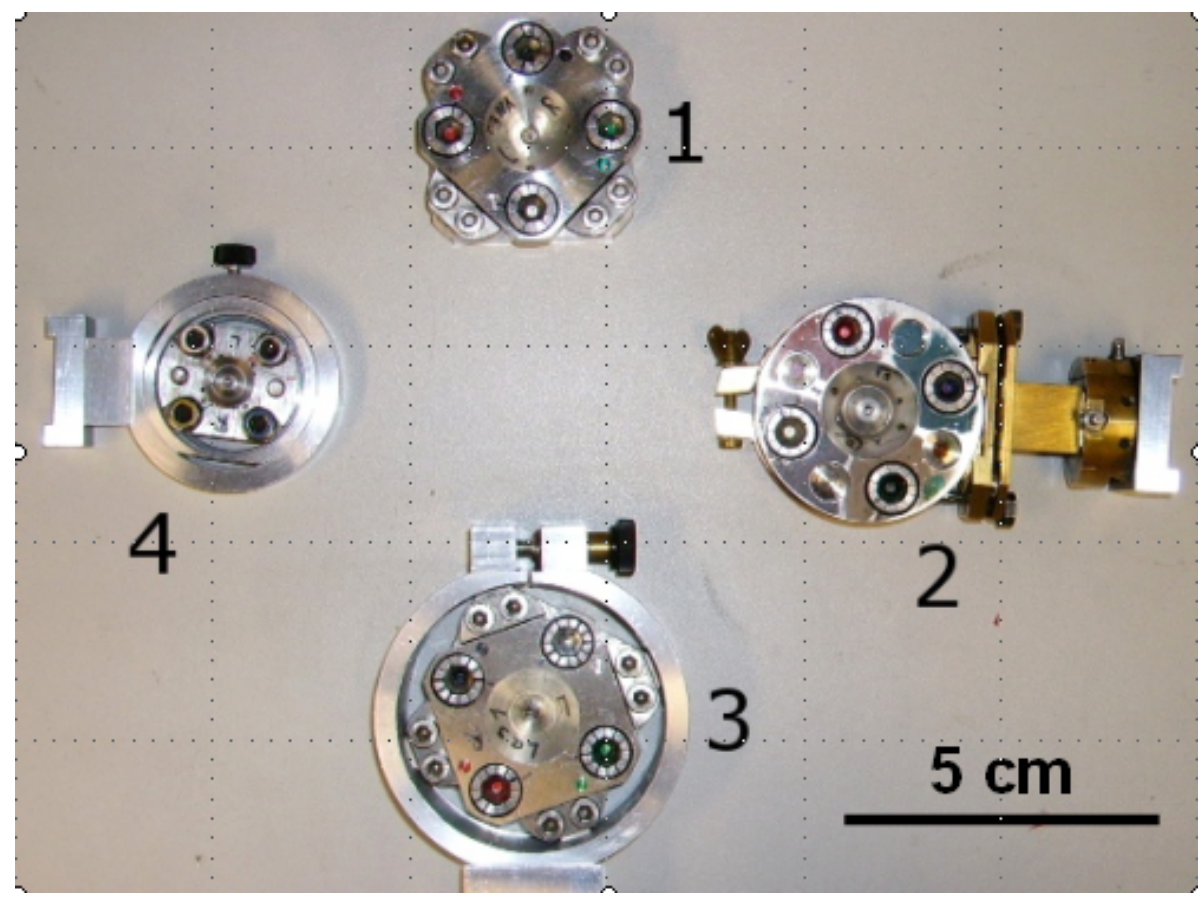

Abbildung 4.1: Übersicht der für die Experimente verwendeten Diamantstempelzellen. 1) 3Dia-Zelle, 2) Runde Vierschraubenzelle (auf Halterung), 3) LAS1-Zelle (auf Halterung), 4) Kleine Vierschraubenzelle (auf Halterung).

Sofern verfügbar, wurden für die Experimente die berylliumfreien DACs bevorzugt, da durch die Berylliumhalterungen zusätzliche störende Pulverringe im Röntgenbeugungsbild entstehen.

Als Gasketmaterial wurde für Maximaldrücke von bis zu 5 GPa Inconel-718-Stahl verwendet, bei höheren Drücken wurde mit einem härteren 2-Phasen-Stahl aus Ferrit und Martensit gearbeitet. Beide Materialien haben eine initiale Dicke von $250 \mu \mathrm{m}$. Im Bereich der späteren Probenkammer wurde die Gasketdicke durch einen Eindruck in der DAC auf 80 bis $90 \mu m$ gebracht. Anschließend wurde mit einem Funkenerosionsbohrer eine Probenkammer mit einem Durchmesser von $300 \mu \mathrm{m}$ gebohrt. Hierbei war es wichtig, möglichst die Mitte des Eindrucks zu treffen, damit die Ränder der Probenkammer gleichmäßig belastet wurden.

Ein Einkristall des Probenmaterials wurde zerbrochen und ein geeignetes Probestück ausgewählt. $\mathrm{Zu}$ den Kriterien der Eignung zählten möglichst saubere Bruchkanten (im Idealfall parallel zu den Spaltflächen), eine Länge und Breite von 150-200 $\mu m$ und eine 

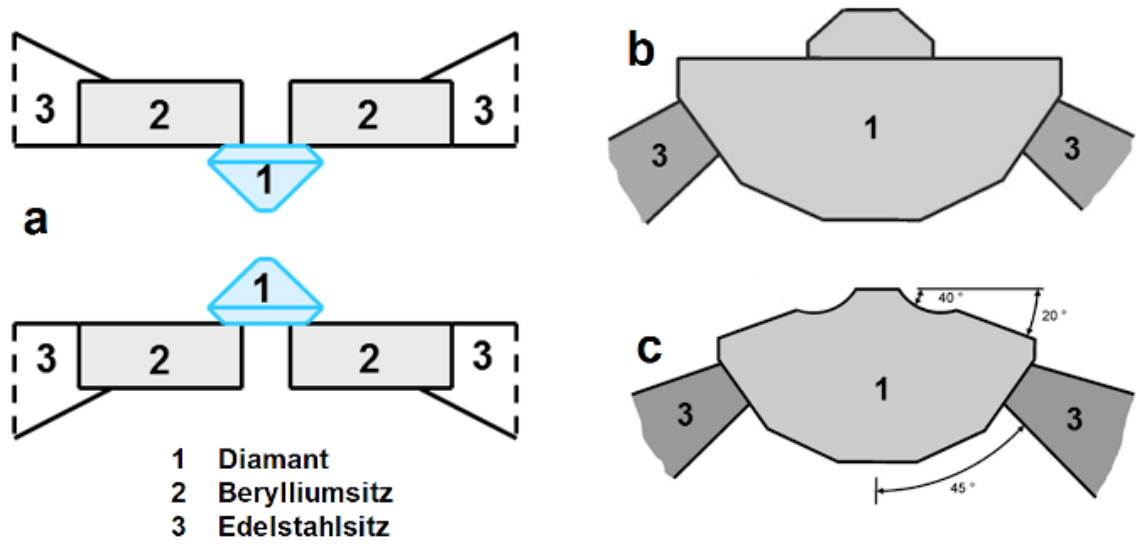

Abbildung 4.2: Typen von Diamantstempelzellen. a) Mao-Bell-Typ mit Berylliumhalterung und aufgeklebten Diamanten (prinzipiell gleicher Aufbau wie Merrill-Bassett-Typ) b) Berylliumfreie Diamanthalterung mit aus zwei Diamanten zusammengesetztem Stempel [4] c) Berylliumfreie Diamanthalterung mit gelasertem Diamanten [74]

Dicke von 30 bis $50 \mu \mathrm{m}$. Das Culet des unteren Diamanten wurde mit einer dünnen Schicht Vaseline bestrichen. Auf dieser wurde die Probe möglichst mittig platziert, sodass sie nach Auflegen des Gaskets innerhalb der Probenkammer lag ohne den Rand der Kammer zu berühren, da der Durchmesser der Probenkammer erfahrungsgemäß im Laufe des Experiments um einige $\mu m$ abnimmt und die Gefahr des Verschiebens der Probe besteht.

Die Messung des in der Probenkammer vorherrschenden Drucks erfolgte mittels der Rubinfluoreszenzmethode. Hierzu wird neben der Probe eine Rubinkugel mit einem Durchmesser von ca. $30 \mu \mathrm{m}$ in die Probenkammer eingesetzt, welcher mit grünem Laserlicht angeregt wird. Die $R_{1}$-Linie des Fluoreszenzspektrums verschiebt ihre Position in einem bekannten Verhältnis zum herrschenden Druck [55]. Der Druck konnte auf 0.05 GPa genau bestimmt werden.

Als druckübertragendes Medium wurde eine Mischung aus Methanol und Ethanol in einem Verhältnis von 4:1 verwendet. Dies gewährt einen hydrostatischen Druck bis zu 10 GPa [54]. Nach Auflegen des Gaskets wurde mit einer Spritze reichlich Druckmedium auf die Probenkammer gegeben. Hierbei musste sehr vorsichtig vorgegangen werden, da die Probe sonst davonschwimmen kann. 
Nach dem Einfüllen des druckübertragenden Mediums wurde die Zelle durch das Aufsetzen des oberen Diamanten und das Anziehen der Schrauben bis zum vollständigen beidseitigen Kontakt mit dem Gasket geschlossen. Dieses muss schnell passieren, da das Druckmedium sehr volatil ist. Anschließend wurde der Druck gemessen. Hatte sich ein geringer Druck aufgebaut und befanden sich keine Luftblasen in der Probenkammer, war die Präparation erfolgreich. Abbildung 4.3 zeigt den schematischen Aufbau der beladenen Diamantstempelzelle.
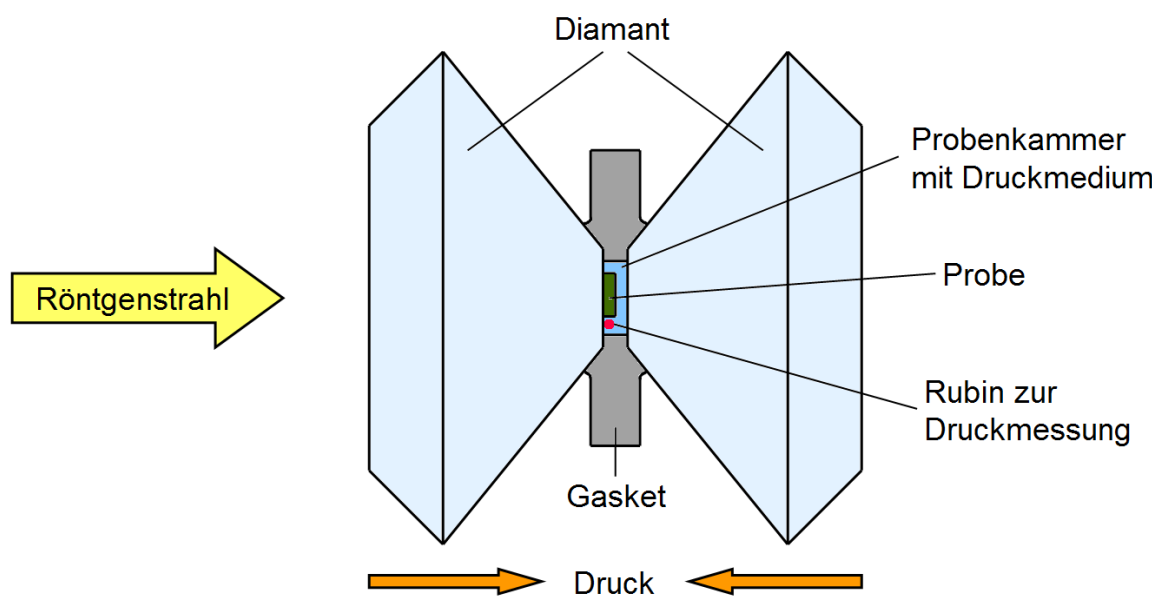

Abbildung 4.3: Schematischer Versuchsaufbau für Hochdruckexperimente in der Diamantstempelzelle

\subsection{Hochtemperaturexperimente}

Für die Hochtemperaturexperimente wurde ein Einkristall des Probenmaterials mit hochtemperaturbeständigem Zement auf einen flachen Probenträger geklebt oder mit einer Feder in einer Halterung festgeklemmt. Eine der Hauptachsen des Kristallgitters lag parallel zur späteren $\omega$-Rotationsachse des Ofens. Die Kristallgröße variierte je nach Verfügbarkeit des Materials zwischen einem Durchmesser von 0.2 bis $2 \mathrm{~mm}$ und einer Länge von 0.5 bis $3 \mathrm{~mm}$.

Die Hochtemperaturexperimente wurden in einem Glaskugelofen durchgeführt. Dieser besteht aus einem auf den Drehtisch des Messplatzes aufsetzbaren Sockel mit den zum Betrieb nötigen Anschlüssen, einer Halterung für die Probe und einer kugelförmigen Glasglocke, die die Probenkammer drastellt. Zum Aufheizen der Probe wird innerhalb der Probenkammer ein bogenförmiges Graphitband über der Probe eingesetzt, das als 
Heizelement dient. (siehe Abbildung 4.4 für ein Bild des Ofens im Betrieb auf der Beamline BW5, HASYLAB). Um den Wärmeverlust so gering wie möglich zu halten, wird während des Heizens ein Vakuum angelegt, das sich im Bereich von $10^{-3}$ bis $10^{-3}$ mbar bewegt.
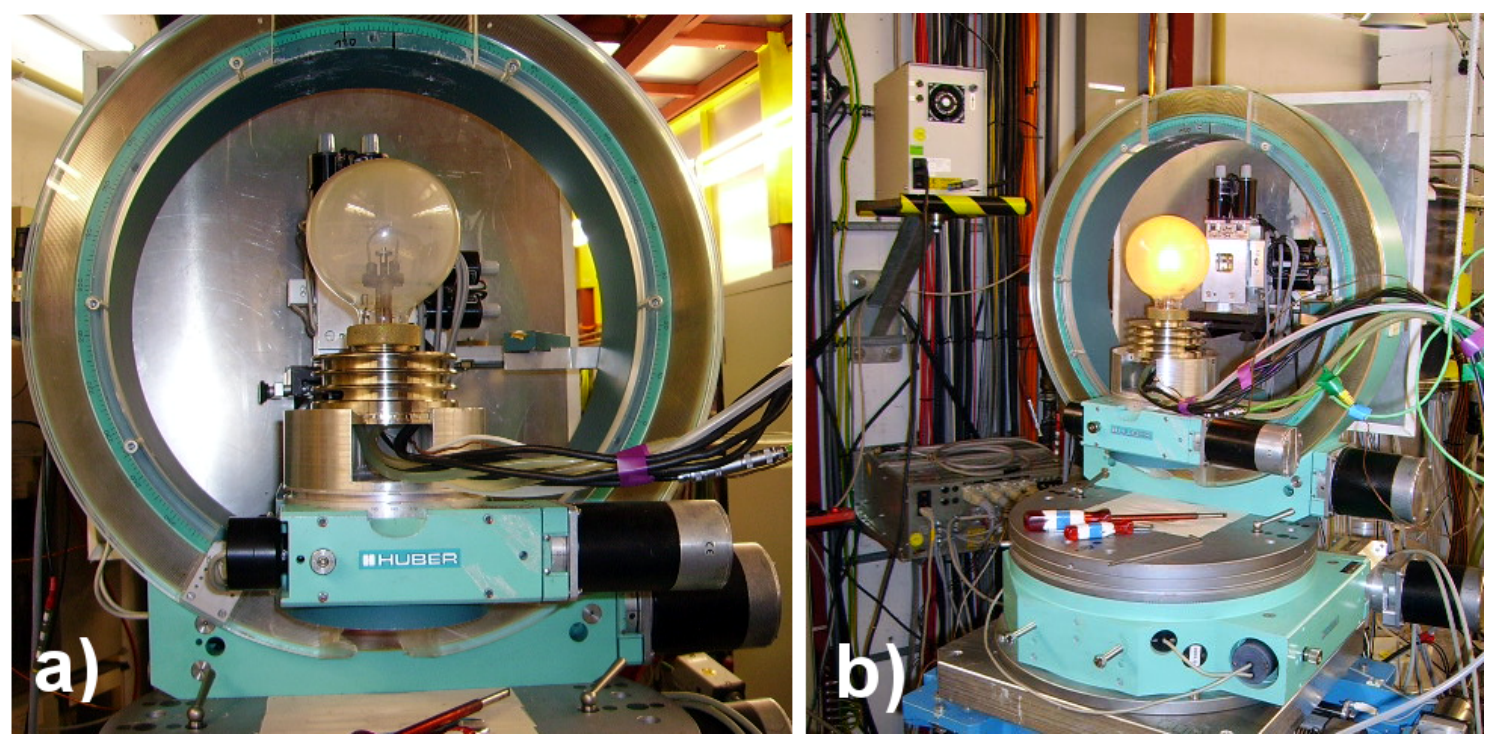

Abbildung 4.4: Versuchsaufbau für Hochtemperaturexperimente an Beamline BW5: a) Mit Probe und Heizband bestückter Ofen auf Drehtisch, im Hintergrund das Schlitzsystem des Primärstrahls; b) Ofen während des Heizvorgangs 


\section{Versuchsdurchführung}

Die Experimente wurden an den Beamlines BW5, W2 und P02.1 im HASYLAB, Hamburg, zwischen August 2007 und November 2013 mit Synchrotronstrahlung durchgeführt, mit einigen vorbereitenden Messungen an konventionellen Röntgengeräten.

\subsection{Erzeugung von Synchrotronstrahlung}

Synchrotronstrahlung hat im Vergleich zu einer Röntgenröhre mehrere Vorteile. Die Intensität der Strahlung ist wesentlich höher, was für das Aufspüren schwacher Probenreflexe von Vorteil ist und aufgrund des geringeren benötigten Stahlquerschnitts eine höhere Ortsauflösung bei der Untersuchung ortsabhängiger Probeneigenschaften erlaubt. Ebenso weist Synchrotronstrahlung einen hohen Photonenfluss (Brillanz) auf. Die extrem geringe Divergenz der Strahlung und die hohe energetische Auflösung führen zu einer scharfen Trennung selbst dicht nebeneinander liegender Probenreflexe. Ebenso kann Synchrotronstrahlung in wesentlich kürzeren Wellenlängen als konventionelle Röntgenstrahlung erzeugt werden. Dies führt einmal dazu, dass vor allem Flächendetektoren aufgrund der geringerem Winkel $\vartheta$ zum Primärstrahl effektiver genutzt werden können und zweitens können durch die höhere Strahlenergie auch dickere Proben durchstrahlt werden.

Werden beschleunigte elementare Ladungsträger in einem Speicherring auf eine gebogene Bahn gelenkt, verlieren sie tangential zu ihrer Beschleunigungsrichtung einen Teil ihrer kinetischen Energie E, der als Synchrotronstrahlung abgestrahlt wird [63]. Dieser Energieverlust hängt vom Radius $R$ des Speicherrings, der Kernladungszahl $Z$, der Energie $E$ des Teilchens, seiner Ruhemasse $m_{0}$ und dem Quotienten $\beta$ aus Teilchen- und 
Lichtgeschwindigkeit ab [5].

$$
\Delta E=\frac{(Z \cdot e)^{2} \cdot \beta^{3}}{\epsilon_{0} \cdot 3 R} \cdot\left(\frac{E}{m_{0} \cdot c^{2}}\right)^{4}
$$

Hierbei beschreibt $e$ die Elementarladung und $\epsilon_{0}$ die elektrische Feldkonstante. Bei relativistischen Geschwindigkeiten nähert sich $\beta$ dem Wert 1 an und Gleichung 5.1 vereinfacht sich zu Gleichung 5.2:

$$
\Delta E=\frac{(Z \cdot e)^{2}}{\epsilon_{0} \cdot 3 R} \cdot\left(\frac{E}{m_{0} \cdot c^{2}}\right)^{4}
$$

Aus den Gleichungen 5.1 und 5.2 ist ersichtlich, dass die Energieausbeute erheblich größer wird, je leichter das Teilchen ist. Deshalb werden in Speicherringen Elektronen oder Positronen zur Erzeugung von Synchrotronstrahlung verwendet.

Bei einem gegebenen Teilchenstrom $I$ im Speicherring ergibt sich die spektrale Verteilung der abgestrahlten Wellenlängen aus der Strahlungsleistung $P$ :

$$
\frac{d P}{d \omega}=\frac{e \cdot \gamma^{4} \cdot I}{3 \epsilon_{0} \cdot R \cdot \omega_{c}} \cdot S_{s}\left(\omega / \omega_{c}\right)
$$

mit $\gamma=E / m_{0} c^{2}$, der kritischen Abstrahlungsfrequenz $\omega_{c}=3 c \gamma^{3} / 2 R$, welche das Spektrum in zwei Bereiche gleicher Strahlungsleistung einteilt, und der Spektralfunktion $S_{s}$. Letztere kann über eine modifizierte Bessel-Funktion gelöst werden. Für eine gegebene Energie wird so Strahlung in einem Intervall von Wellenlängen erzeugt, deren Intensität zu den Rändern dieses Intervalls hin abnimmt.

Der Winkel $\theta$ zwischen der momentanen Flugbahn eines Teilchens und dem Maximum der Abstrahlung der erzeugten Synchrotronstrahlung ist gegeben durch Gleichung 5.4:

$$
\tan \theta=\frac{1}{\gamma}=\frac{m_{0} c^{2}}{E}
$$

Je höher die Teilchengeschwindigkeit, desto mehr nähert sich $\gamma$ dem Wert 1 und desto kleiner wird $\theta$. Gleichzeitig verschmälert sich das Intervall um $\theta$, welches das abgestrahlte Wellenlängenintervall beinhaltet. Im Ring befinden sich Magnetstrukturen, Wiggler oder Undulatoren, in denen die weiße Röntgenstrahlung entsteht. beide unterscheiden sich in der Größe des 'Brennflecks' und liefern daher unterschiedliche räumliche Intensitätsverteilungen. Durch ein Strahlrohr wird die Synchrotronstrahlung vom Speicherring in die Messhütte zum Experiment geleitet. Ein Monochromatorkristall im Strahlrohr 
ermöglicht es, aus der weißen Synchrotronstrahlung die für das Experiment gewünschte Wellenlänge herauszufiltern und so monochromatische Röntgenstrahlung zu erzeugen.

\subsection{Beamlines BW5 und W2, DORIS III}
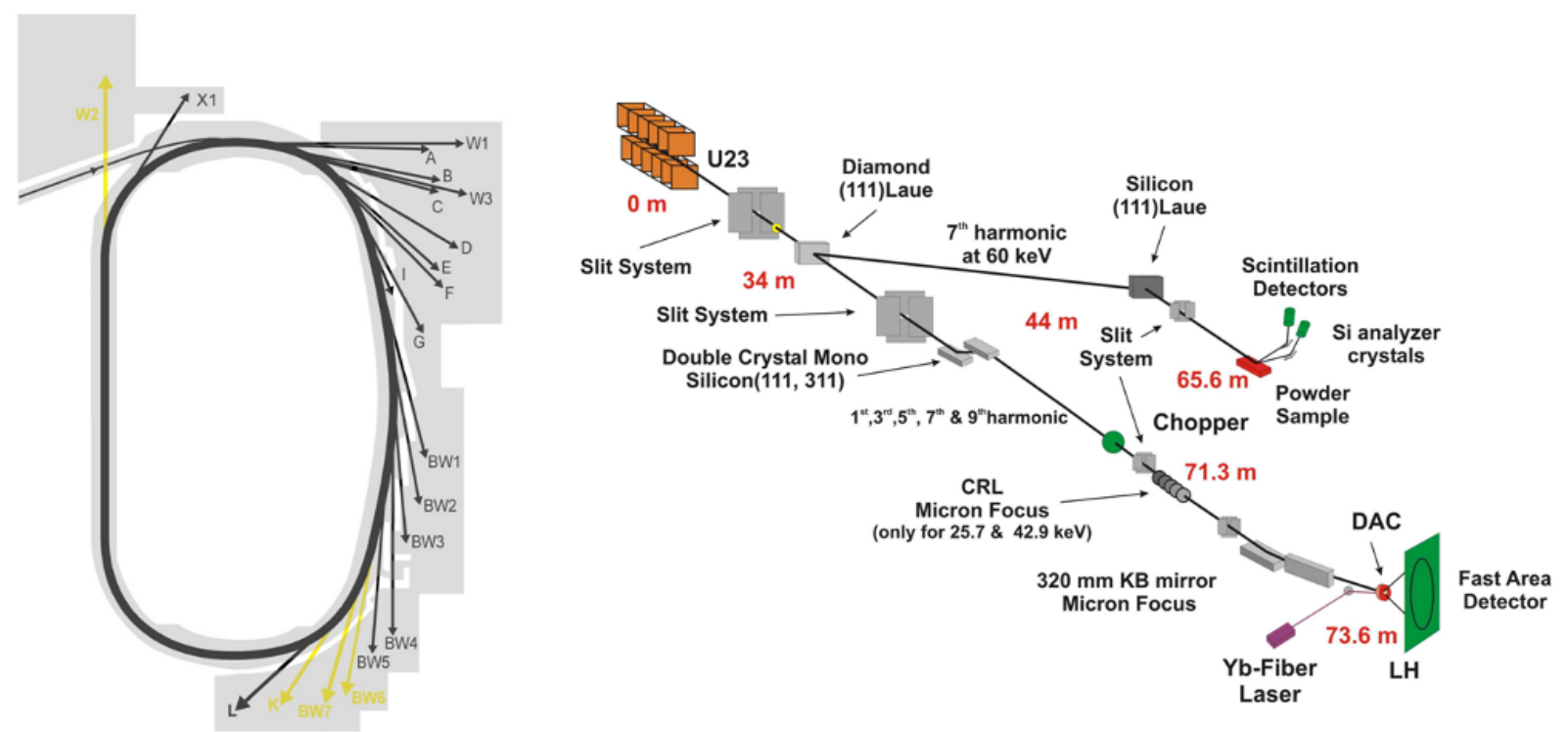

Abbildung 5.1: Links: Schema des Speicherrings DORIS III mit allen abzweigenden Beamlines. Rechts: Schema des Aufbaus der Beamline P02 am Speicherring PETRA III. Die Beamline P02.1 stellt den oberen Zweig dar. (Quelle: DESY Pressematerial)

DORIS ist ein ovaler Teilchenbeschleunigerring mit einem Umfang von 289 Metern, der ursprünglich für Anwendungen der Kern- und Teilchenphysik konzipiert war. Die ersten Experimente mit Synchrotronstrahlung wurden im Jahr 1974 durchgeführt, im Jahr 1981 wurde das Hamburger Synchrotronstrahlungslabor (HASYLAB) gegründet. Nach einem Umbau im Jahr 1992 wurde die Einrichtung DORIS III getauft. Im Oktober des Jahres 2012 wurde der Betrieb des Speicherrings eingestellt.

Basis der Beamline BW5 ist ein Wiggler innerhalb des DORIS-Speicherrings mit einer Energie von $26 \mathrm{keV}$, der der Beamline auch ihren Namen gibt (Beamline Wiggler 5) [87]. Durch eine lineare Anordnung von Dipolmagneten, die die Elektronen zusätzlich zu ihrer Kreisbahn durch den Beschleunigerring zu kleinräumigen Sinusschwingungen zwingen, wird auf diese Weise die Strahlungsausbeute erhöht. Die vom Wiggler erzeugte Röntgenstrahlung wird durch einen Kollimator und ein Schlitzsystem gebündelt und über 
das Strahlrohr in die Messhütte geleitet. Hinter einem weiteren Kollimator befindet sich eine Auswahl von Si- und Si-Ge-Monochromatoren, mit denen monochromatische Röntgenstrahlung mit einer Strahlenergie von 60 bis $250 \mathrm{keV}$ aus dem weißen Primärstrahl herausgefiltert werden kann. Für die vorliegenden Experimente wurde eine Energie von $100 \mathrm{keV}$ verwendet. Dies entspricht einer Wellenlänge von $0.12 \AA$. Auf den Monochromator folgen ein Shutter für die Belichtungszeit, ein Absorber, mit dem die Strahlintensität geregelt wird, und ein doppeltes Schlitzsystem, mit dem Breite und Höhe des Strahls, der schlussendlich auf die Probe trifft, variiert werden können. Im Fall der vorliegenden Experimente lag der so erzeugte Strahlquerschnitt zwischen 0.5 und $1 \mathrm{~mm}$. Die Druckzelle bzw. der Ofen mit der Probe ist auf einer Eulerwiege oder einem Drehtisch mit Translationsvorrichtung befestigt. Hinter dem Probentisch befindet sich ein Flächendetektor, mit welchem das von der Probe erzeugte Beugungsbild aufgenommen wird. Der Abstand zwischen Probentisch und Detektor variierte zwischen 1200 und $1500 \mathrm{~mm}$, war jedoch für den Zeitraum einer Messexkursion immer auf einen festen Wert festgelegt.

Die Daten der zwischen August 2007 und September 2010 durchgeführten Experimente wurden mit einem mar345 Image Plate-Detektor mit einem Plattendurchmesser von 345 mm aufgenommen. Die verwendete Auflösung betrug 150x150 $\mu \mathrm{m}$ pro Pixel bei einer maximalen Bildgröße von 2300x2300 Pixeln. Die Bildgröße ließ sich in 4 Schritten bis auf 1200x1200 Px verkleinern (Marmodes 0 bis 3) [1]. Von Mai 2011 an wurde ein Perkin Elmer 1621 Halbleiter-TFT-Detektor mit einer Fläche von 410x410 mm zur Datenaufnahme verwendet. Die Auflösung beträgt 200x200 $\mu \mathrm{m}$ pro Pixel bei einer Bildgröße von 2048x2048 Pixeln.

Das Auffüllen des Speicherrings DORIS erfolgt im Sechs-Stunden-Rhythmus. Dies bedeutet, dass die im Ring vorherrschende Stromstärke und damit die Intensität des Strahls zwischen den einzelnen Füllungen exponentiell abfallen. Die Strahlintensität schwankt bei Normalbetrieb des Speicherrings zwischen ca. $140 \mathrm{~mA}$ direkt nach der Füllung und ca. $100 \mathrm{~mA}$ am Ende eines Zyklus. Der Aufbau der Beamline W2 entspricht dem Aufbau der Beamline BW5. Der Messplatz ist jedoch für wesentlich voluminösere und schwerere Proben ausgerichtet. 


\subsection{Beamline P02.1, PETRA III}

Der ringförmige Teilchenbeschleuniger PETRA III hat eine Länge von 2304 Metern und nahm seinen Betrieb in der heutigen Konfiguration als Synchrotronstrahlungsquelle im Jahr 2009 auf. Zuvor diente er als Vorbeschleuniger für den 2007 stillgelegten HERATeilchenbeschleuniger.

Im Gegensatz zu DORIS werden dem Speicherring kontinuierlich Teilchen im Top-UpMode zugeführt, sodass die Stromstärke im Ring konstant 100 mA beträgt. Die Synchrotronstrahlung der Beamline P02 wird von einem Hochenergie-Undulator im Speicherring erzeugt. Ein Undulatur ist eine Weiterentwicklung des Wigglers, die sich durch die Erzeugung einer höheren Strahlbrillanz und eine geringere Wellenlängenstreuung auszeichnet. Die so erzeugte Strahlung wird durch ein Schlitzsystem mit einer Apertur von 1mm*1mm auf einen Diamant-Lauemonochromator geleitet. Dieser spaltet den Strahl für die einzelnen Beamlines P02.1 und P02.2 auf, wobei die Strahlung, die einer Energie von $60 \mathrm{keV}$ entspricht, der Beamline P02.1 zugeteilt wird [20]. Dies führt zu einer allzeit konstanten Wellenlänge von $0.207 \AA$. Ein zweiter, aus Silizium bestehender Monochromator richtet den nun monochromatischen Strahl in das Stahlrohr der Messhütte aus, wo durch ein zweites Schlitzsystem sein Energieprofil gesteuert werden kann. Ein drittes Schlitzsystem mit einem Absorber befindet sich innerhalb der Messhütte. Hiermit lassen sich Durchmesser, Form und Intensität des Strahls variieren. Die Probe befindet sich auf einem Drehtisch mit waagerecht angeordneter $\omega$-Achse. Als Detektor dient ein Perkin Elmer 1621 Halbleiter-TFT-Detektor mit den gleichen Spezifikationen wie an der Beamline BW5, der zur Variation des Probe-Detektor-Abstands auf einem Schlitten auf Schienen gelagert ist und mit einem Schrittmotor verfahren werden kann. So sind Probe-DetektorAbstände von $800 \mathrm{~mm}$ bis $1800 \mathrm{~mm}$ möglich, bei zusätzlicher Nutzung der sich auf dem Schlitten befindenden Verfahrmöglichkeiten sogar Probe-Detektor-Abstände von $200 \mathrm{~mm}$ bis $3000 \mathrm{~mm}$.

\subsection{Hochdruckexperimente}

Die mit dem Einkristall geladene Druckzelle wurde in einer verrutschsicheren Halterung auf dem Drehtisch montiert und so justiert, dass die Probe auf der $\omega$-Achse des Drehtischs lag. Aufgrund der scharfen Reflexe eines Einkristalls wurde die Orientierungsmessung 
des Einkristalls mit $\Delta \omega$-Schritten von $0.5^{\circ}$ oder kleiner durchgeführt. Anschließend wurde der Druck erhöht, bis die Umwandlung in die Hochdruckmodifikation abgeschlossen war. Dies wurde durch Einzelbildmessungen überprüft. Nach erfolgreicher Umwandlung wurde die Textur der Hochdruckmodifikation in zwei verschiedenen Ausrichtungen der Druckzelle gemessen. Da der durch die Druckzellengeometrie bedingte maximale Öffnungswinkel der Druckzelle keine vollständige Abdeckung des reziproken Raumes und somit keine vollständigen Polfiguren zulässt, sorgt dieses Verfahren für eine bessere Datenausbeute (vgl. Kap. 6.2). Die erste Messung erfolgte in der gleichen Zellenorientierung wie die Einkristallmessung, anschließend wurde die Druckzelle um $90^{\circ}$ um die Achse des Röntgenstrahls gedreht und die Messung unter den gleichen Bedingungen wiederholt. Anschließend wurde der Druck in der Probenkammer schrittweise verringert, bis die Rückumwandlung der Probe in die Niederdruckmodifikation abgeschlossen war, und das gleiche Messprinzip wie bei der Hochdruckmodifikation nochmals angewandt.
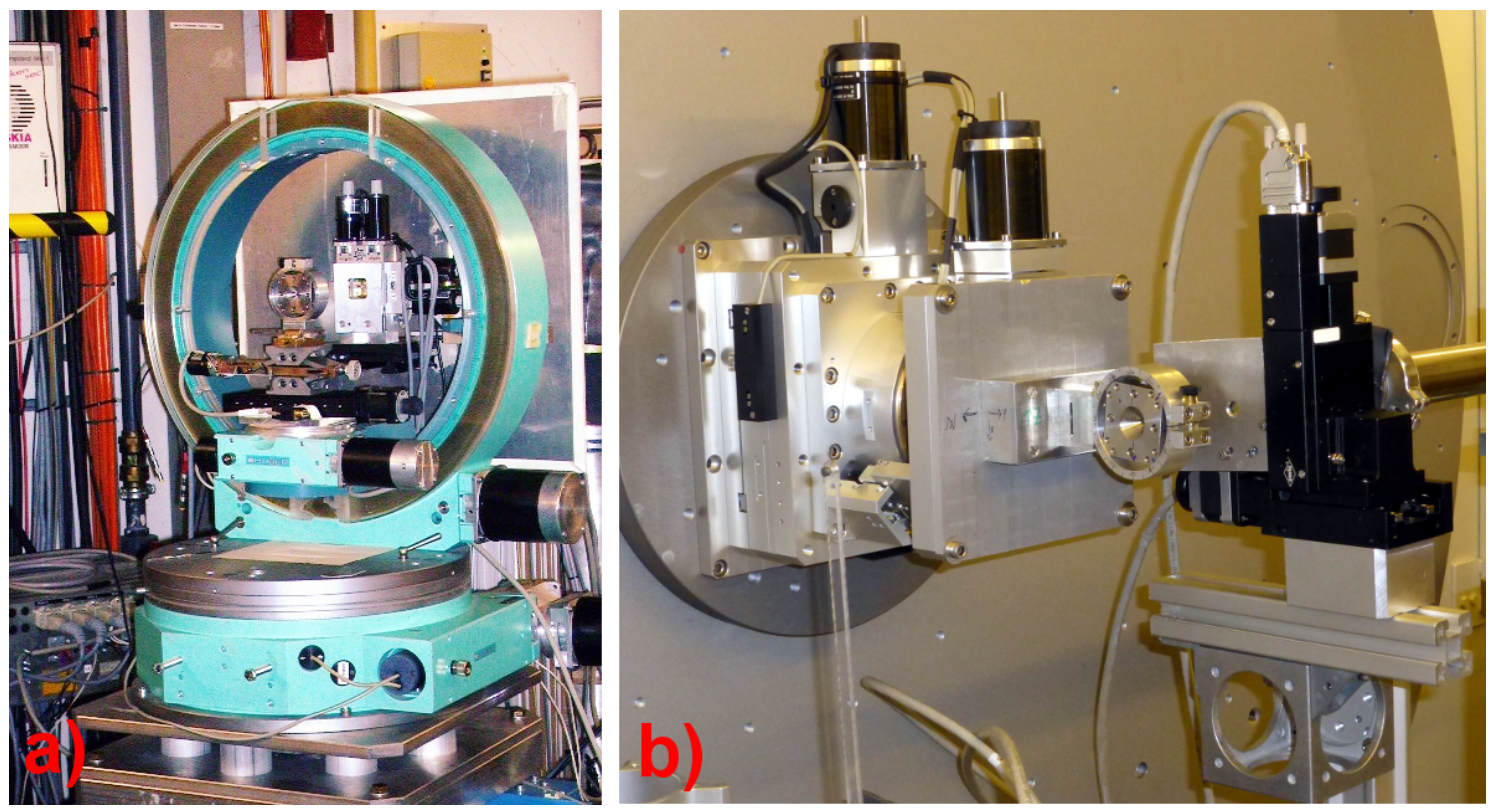

Abbildung 5.2: Versuchsaufbau zur Messung von Hochdruckexperimenten: a) Beamline BW5, vertikale $\omega$-Achse; b) Beamline P02.1, horizontale $\omega$-Achse.

\subsection{Hochtemperaturexperimente}

Der Sockel des Glaskugelofens wurde auf den Drehtisch montiert und der Probenhalter eingesetzt. Nach der Justierung der Probe auf die $\omega$-Achse des Drehtischs wurde eine 
Orientierungsbestimmung des Einkristalls durchgeführt. Da sich der Ofen frei drehen ließ, konnte der gesamte reziproke Raum abgedeckt werden. Anschließend wurde ein bogenförmiges Graphitband über der Probe eingesetzt, das als Heizelement diente, und die Glaskugel aufgesetzt. Abbildung 4.4 in Kapitel 4.2 zeigt den Glaskugelofen im Betrieb auf dem Messplatz BW5 im HASYLAB. Die Probe wurde langsam erhitzt bis die Phasenumwandlung eintrat. Das Aufheizen der Proben erfolgte zuerst schnell, anschließend ab etwa $100^{\circ} \mathrm{C}$ unter der Umwandlungstemperatur in $5^{\circ} \mathrm{C}$ - bis $10^{\circ} \mathrm{C}$-Schritten. Zwischen den einzelnen Aufheizschritten wurde jeweils ein Detektorbild angefertigt um zu erkennen, ob die Umwandlung schon vollständig stattgefunden hatte. Anschließend wurde eine weitere Orientierungsmessung durchgeführt. Da die Probenmaterialien ihre Hochtemperaturmodifikation auch metastabil bei Raumtemperatur beibehielten, war keine Messung einer polykristallinen Tieftemperaturmodifikation möglich. Allerdings ermöglichte diese Tatsache eine Messung der Orientierung der Hochtemperaturmodifikation ohne die störenden Effekte von Glasglocke und Graphitband auf den Detektorbildern. 


\section{Auswertung}

\subsection{Vom Detektorbild zur Polfigur}

Das Bild eines Flächendetektors zeigt die Reflexe des Materials je nach Ausprägung der Textur als Ringe, linsenförmige Objekte oder Punkte. Zur Erstellung einer Polfigur wird für alle Detektorbilder ein Ring mit festem $2 \vartheta$-Winkel ausgeschnitten und die Intensität des Reflexes entlang des Ringes im Winkel $\gamma$ über den gesamten Ring ausgelesen [15]. So ergibt sich für jeden Punkt entlang des Rings ein Diffraktionsvektor $\vec{s}(\gamma, \vartheta)$ zwischen dem Primärstrahl und dem reflektierten Strahl im Koordinatensystem des verwendeten Messgeräts (Abbildung 6.1).

Um die gemessenen Intensitäten in das Probenkoordinatensystem $K_{A}$ und somit in die Polfigur zu übertragen, muss der Diffraktionsvektor $\vec{s}(\gamma, \vartheta)$ über eine Transformationsmatrix $G_{i k}$ in die Probenrichtung $\vec{y}(\alpha, \beta)$ umgewandelt werden (Gleichung 6.1).

$$
\vec{y}_{i}=G_{i k} \cdot \vec{s}_{k}
$$

mit

$$
y_{i}=\left(\begin{array}{c}
\sin \alpha \cdot \cos \beta \\
\cos \alpha \\
\sin \alpha \cdot \sin \beta
\end{array}\right), s_{k}=\left(\begin{array}{c}
-\sin \vartheta \\
\cos \vartheta \cdot \cos \gamma \\
\cos \vartheta \cdot \sin \gamma
\end{array}\right)
$$

und der Transformationsmatrix

$$
G_{i k}=\left(\begin{array}{ccc}
\cos \omega \cos \varphi-\sin \omega \sin \varphi \cos \chi & \sin \omega \cos \varphi+\cos \omega \sin \varphi \cos \chi & \sin \varphi \sin \chi \\
-\cos \omega \sin \varphi-\sin \omega \cos \varphi \cos \chi & -\sin \omega \sin \varphi+\cos \omega \cos \varphi \cos \chi & \cos \varphi \sin \chi \\
\sin \omega \sin \chi & -\cos \omega \sin \chi & \cos \chi
\end{array}\right)
$$

Die Winkel $\omega, \varphi$ und $\chi$ bezeichnen die Eulerschen Winkel. Für die vorliegenden Mes- 


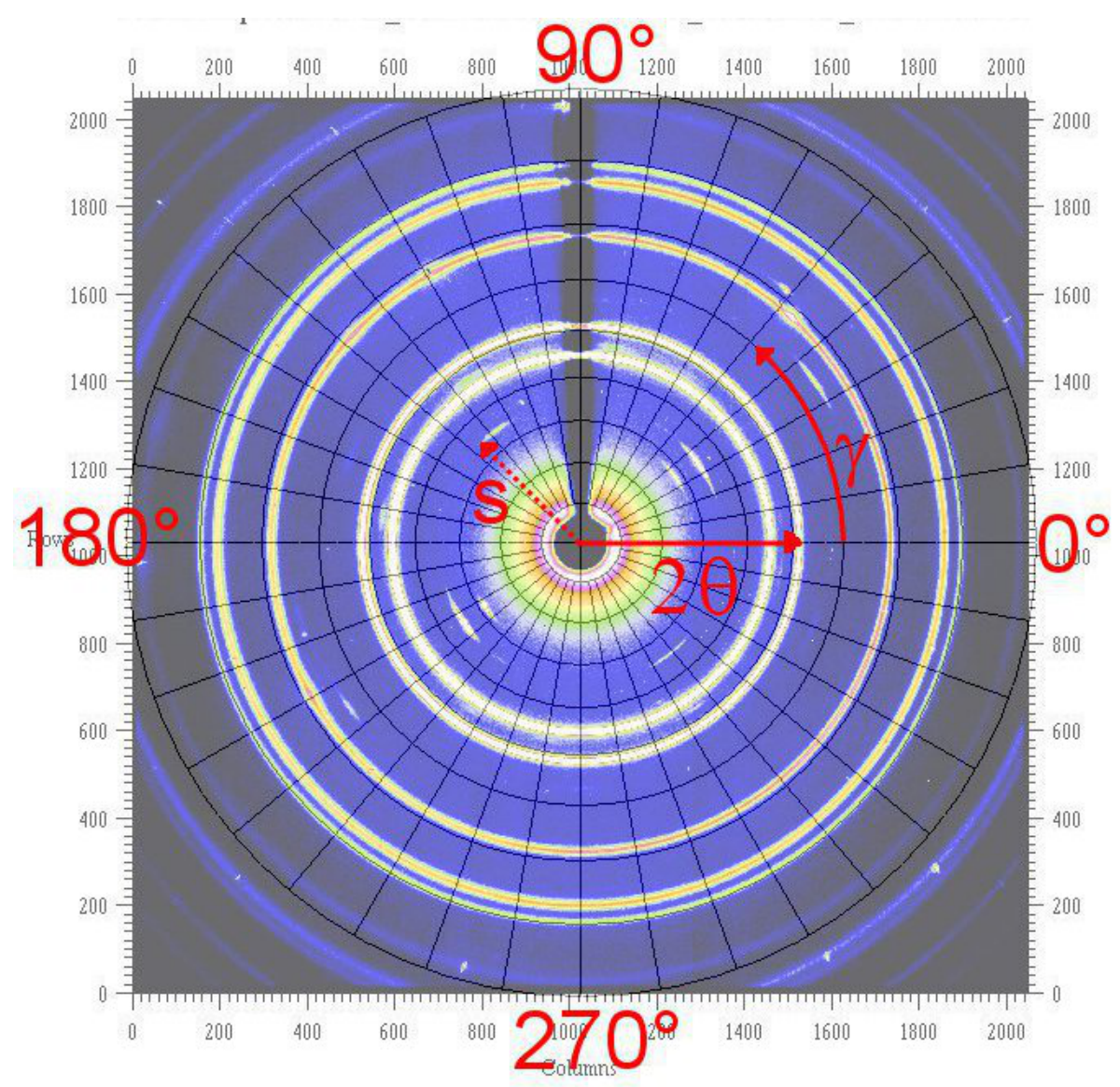

Abbildung 6.1: Auslesen der Winkel $\gamma$ und $2 \vartheta$ sowie des Vektors $\vec{s}$ aus einem Detektorbild. Hier: Koexistierende Hochdruck- und Niederdruckphase von InAs.

sungen wurde lediglich der $\omega$-Winkel variiert. Die Winkel $\varphi$ und $\chi$ wurden Null gesetzt. Hiermit vereinfacht sich $G_{i k}$ stark und Gleichung 6.1 wird zu Gleichung 6.4.

$$
\left(\begin{array}{c}
\sin \alpha \cdot \cos \beta \\
\cos \alpha \\
\sin \alpha \cdot \sin \beta
\end{array}\right)=\left(\begin{array}{ccc}
\cos \omega & \sin \omega & 0 \\
-\sin \omega & \cos \omega & 0 \\
0 & 0 & 1
\end{array}\right) \cdot\left(\begin{array}{c}
-\sin \vartheta \\
\cos \vartheta \cdot \cos \gamma \\
\cos \vartheta \cdot \sin \gamma
\end{array}\right)
$$

Da die Abhängigkeit vom Winkel $\vartheta$ zu größeren Werten hin die Polfigur immer stärker verzerrt, wird anstelle von $\omega$ der Winkel $\omega-\vartheta$ verwendet.

Um die Polfigurwinkel $\alpha$ und $\beta$ zu erhalten, lässt sich das Gleichungssystem 6.4 auf drei verschiedene Weisen lösen. Der Lösungsweg hat lediglich Einfluss darauf, welche Quadranten des Detektorbilds auf der oberen und der unteren Hälfte der Lagenkugel der stereographischen Projektion abgebildet werden. Für das Endergebnis spielt es jedoch keine Rolle, sofern der Lösungsweg für alle Messungen eines Experiments der gleiche 
bleibt. Beispielhaft sei hier der Weg über die Werte $y_{1}$ und $y_{2}$ gezeigt. Aus Gleichung 6.4 ergibt sich durch Lösen der rechten Seite:

$$
\begin{array}{r}
y_{1}=\sin \alpha \cdot \cos \beta=-\sin \vartheta \cdot \cos \omega+\cos \vartheta \cdot \cos \gamma \cdot \sin \omega \\
y_{2}=\cos \alpha=\sin \vartheta \cdot \sin \omega+\cos \vartheta \cdot \cos \gamma \cdot \cos \omega
\end{array}
$$

Auflösen von Gleichung 6.6 nach $\alpha$ und Einsetzen in Gleichung 6.5 ergibt:

$$
\begin{array}{r}
\alpha=\arccos \left(y_{2}\right) \\
\beta=\arccos \left(\frac{y_{1}}{\sin \alpha}\right)
\end{array}
$$

Die aus den Gleichungen 6.7 und 6.8 ermittelten $\alpha$ - und $\beta$-Werte werden für alle verfügbaren $\omega$ - $\gamma$ - und $\vartheta$-Werte im Wulffschen Netz aufgetragen und ergeben in ihrer Gesamtheit eine Polfigur. Die so ermittelten experimentellen Polfiguren können nun zur Berechnung der ODF eingesetzt werden.

\subsection{Auswertung}

Zur Auswertung der Daten der Hochtemperaturmodifikation von NiS wurde das von Dr. Luca Lutterotti entwickelte Programm MAUD [43] verwendet, das sowohl eine RietveldVerfeinerung [58] der Daten als auch eine Berechnung der Textur nach der in Kapitel 2.5.3 beschriebenen E-WIMV-Methode leisten kann.

Der aus 2D-Detektorbildern bestehende Rohdatensatz wurde mit dem von Dr. Lars Raue entwickelten Programm input4Maud [57] unter Eingabe der Messparameter in ein für MAUD lesbares Format umgewandelt. Hierbei wird jedes einzelne Detektorbild entlang des Winkels $\gamma$ in Tortenstücke mit Schrittweite $\Delta \gamma$ unterteilt. Somit wird für jedes Detektorbild des Datensatzes beim Einlesen in MAUD eine durch $\Delta \gamma$ festgelegte Anzahl von Diffraktogrammen erstellt, die jeweils die über $\Delta \gamma$ integrierten Reflexintensitäten enthalten.

Zunächst wird für jedes Diffraktogramm eine Rietveld-Verfeinerung ohne Berücksichtigung der Textur durchgeführt. Dies führt zu einem über die gesamte Menge der Diffraktogramme gemittelten Fit, der bei einzelnen Diffraktogrammen je nach Ausprägung der Textur starke Abweichungen von den tatsächlichen Peakhöhen zeigen kann.

Um die Differenz zwischen den durch die Rietveld-Verfeinerung erhaltenen Peakhöhen 
und den tatsächlichen Peakhöhen der einzelnen Diffraktogramme zu erhalten, wird für jedes Diffraktogramm eine Peakprofilanalyse nach Le Bail [39] durchgeführt. Aus den vorhandenen Informationen werden experimentelle Polfiguren erstellt, deren durch die Peakprofilanalyse nach Le Bail erhaltenen Texturgewichtungsfaktoren als Startwerte für die anschließende iterative Verfeinerung der Textur nach der E-WIMV-Methode dienen. Nach Abschluss des Verfeinerungsprozesses wurde die ODF ausgegeben und die rückgerechneten Polfiguren erstellt. Die Darstellung der ODF erfolgte mit dem Programm BEARTEX [88].

Die Einkristalldaten wurden anlog zum Auswertungsweg der Hochdruckexperimente ausgewertet.

Im Zuge der Auswertung stellte sich heraus, dass das Programm MAUD [43] nicht zur Auswertung von Proben mit sehr starker Textur geeignet ist. Deshalb ließen sich sämtliche Einkristalldatensätze nicht mit diesem Programm auswerten. Ebenso ergaben sich Schwierigkeiten bei der Auswertung der polykristallinen Materialien in den Druckzellen, da die Probenreflexe im Vergleich zu den Reflexen des Gasket- und Zellenmaterials nur schwach ausfallen (Siehe Abbildung 6.2). Hier versagte MAUD dabei, wichtige Details der Textur zu erfassen oder erkannte die Textur erst gar nicht.

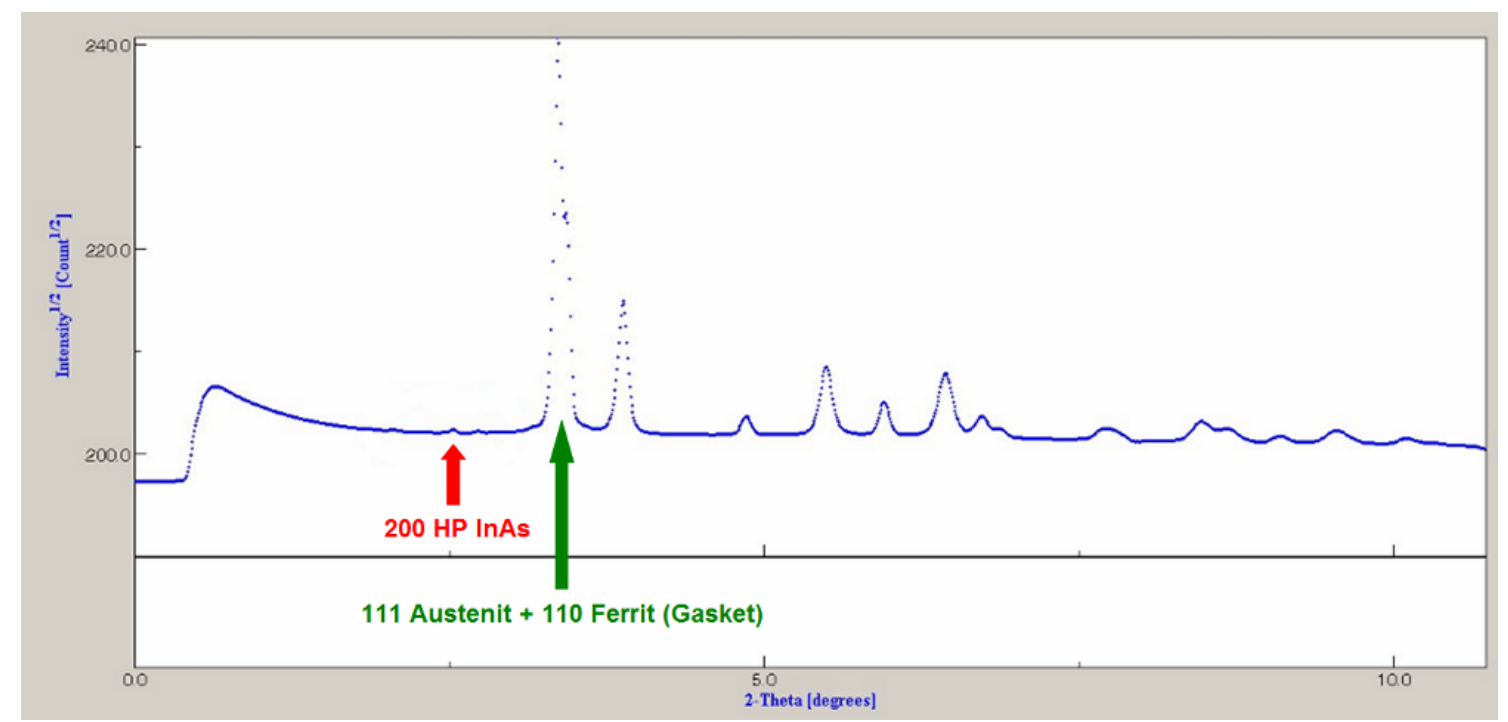

Abbildung 6.2: Diffraktogramm der Hochdruckphase von Indiumarsenid, dargestellt in MAUD, Vergleich der Peakhöhen der Probenmaterial- und der Gasketmaterialreflexe.

Bei manchen Substanzen (z. B. CuInSe $)_{2}$ kam es vor, dass mehrere Probenreflexe sehr 
dicht neben oder sogar auf einem der Ringe der Zellmaterialreflexe lagen (Abbildung 6.3a), was aufgrund der geringen Intensität der Probenreflexe von MAUD nicht zu erkennen war. Deshalb konnte Maud schließlich nur für die Auswertung der polykristallinen Phasen der Hochtemperaturexperimente verwendet werden.

Durch die starken Reflexe der Diamantstempel kam es stellenweise zum Blooming-Effekt (Abbildung 6.3b), wo ein Diamantreflex die gesamte Umgebung bei mehreren hintereinander folgenden Detektorbildern überstrahlte und damit auch die sich dort befindenden Probenreflexe unsichtbar werden ließ. Aufgrund der teilweisen Abschattung des Strahls durch das Druckzellenmaterial bei hohen $\omega$-Winkeln ließ sich oft nicht der komplette 90-Öffnungswinkel der Druckzelle für die Messung ausnutzen, was zu einer noch geringeren Abdeckung der gemessenen Polfiguren führte. Aufgrund der begrenzten Messzeit an den Beamlines war es auch nicht möglich, Experimente zu wiederholen, die sich in der Auswertung als misslungen herausstellten.
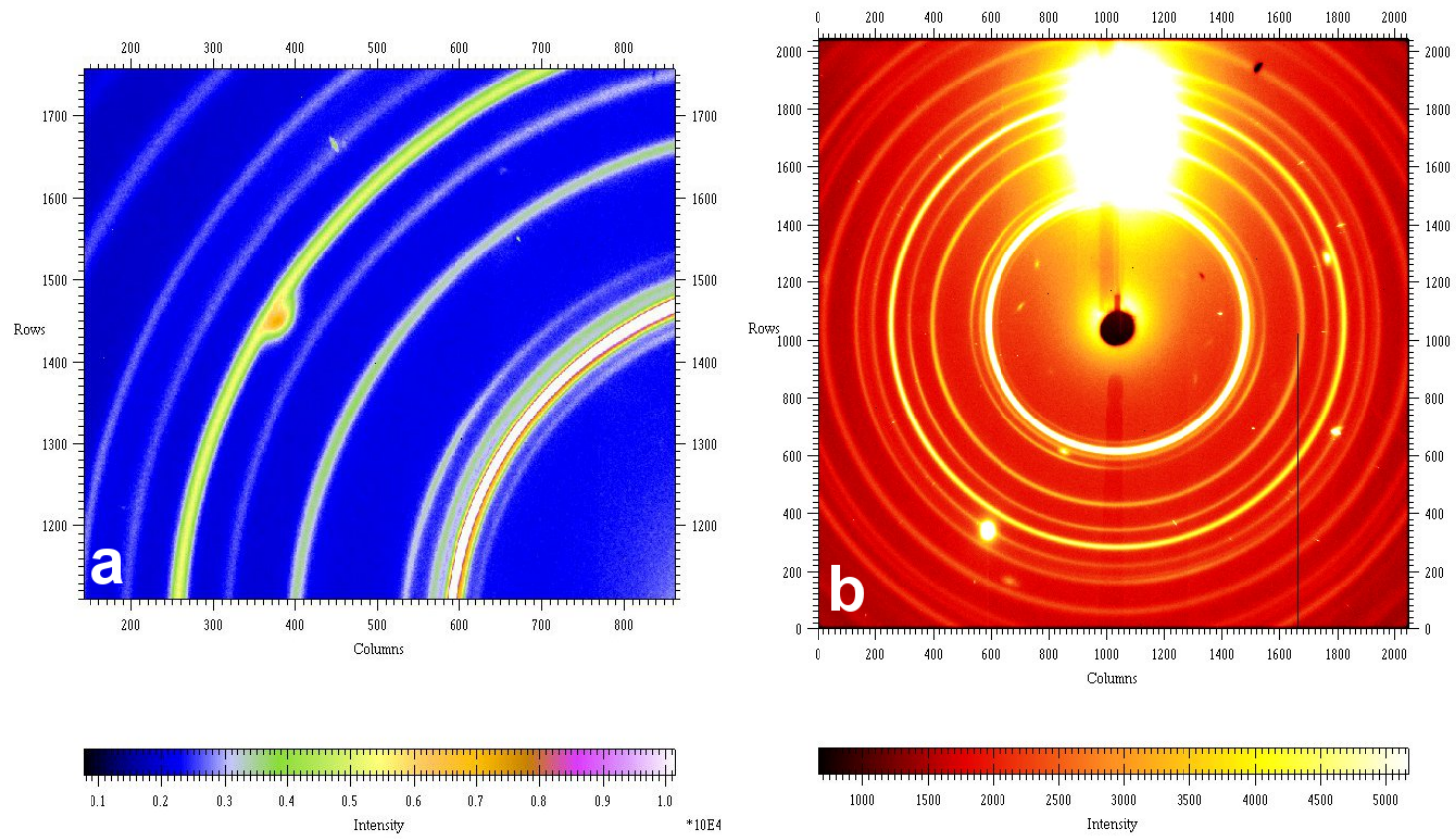

Abbildung 6.3: a) Überlappung von Proben- und Gasketmaterialreflex, b) Blooming-Effekt. Beide Beispiele an $\mathrm{CuInSe} \mathrm{S}_{2}$.

Die Auswertung der Hochdruckexperimente begann mit dem Indizieren der gemessenen Reflexe im Programm Fit2D [28],[29] und dem Festlegen von minimalem und maximalem $2 \vartheta$-Wert der auszuschneidenden Ringe. Wenn möglich wurde für jede zu analysierende Reflexschar ein Untergrund gesetzt um das Hintergrundrauschen des Detektors und an- 
dere Störeffekte, wie zum Beispiel Reflexe der Gasket- und Druckzellenmaterialien, zu minimieren. Die in Fit2D ermittelten Werte wurden zusammen mit den Parametern des Experiments in das Programm Image 2 Pf (L. Raue, unveröffentlicht) eingegeben. Hierbei wurde eine Intervallbreite $\Delta \gamma$ festgelegt, über die die Intensitäten integriert werden. Sinnvolle $\Delta \gamma$-Werte sind je nach Schärfe der Textur $1^{\circ}$ bis $5^{\circ}$. Die so berechneten Intensitäten entlang des ausgeschnittenen Rings wurden in Form einer Tabelle ausgegeben. Zum Abziehen des Untergrunds und zur Kombination mit den um $90^{\circ}$ versetzt gemessenen Daten wurde das Programm LSTADD (H. Klein, unveröffentlicht) verwendet. Abbildung 6.4 zeigt, wie die $0^{\circ}$ - und $90^{\circ}$-Datensätze zum Zweck einer größeren Abdeckung des messbaren Polfigurbereichs kombiniert wurden.

Die so aufbereiteten Daten wurden in das Programm PoleFigureInterpolator (C. Ran-

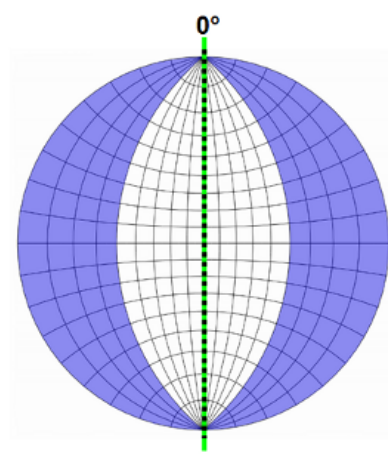

$\omega$-Achse parallel zu ausgewählter Kristallrichtung

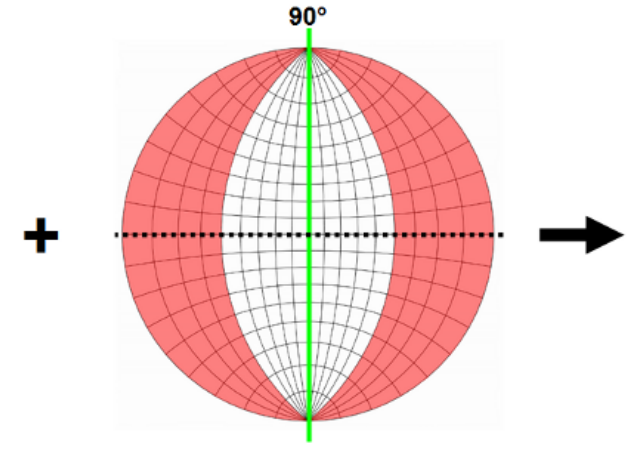

$\omega$-Achse senkrecht auf ausgewählte Kristallrichtung

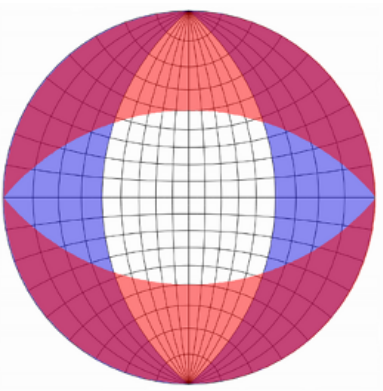

Größerer messbarer Polfigurenbereich

Abbildung 6.4: Kombination zweier um die Strahlachse rotierter Polfiguren zum Erhalt eines größeren messbaren Polfigurbereichs. Die grüne Linie stellt die jeweilige $\omega$-Achse der Eulerwiege dar, die gestrichelte Linie die als $0^{\circ}$ ausgezeichnete Kristallrichtung während der Messung. Die farbigen Bereiche kennzeichnen den jeweils messbaren Teil der Polfigur.

dau, unveröffentlicht) eingelesen, welches aus den Daten die unvollständige gemessene Polfigur erstellt und durch Interpolation basierend auf den Messdaten eine vollständige Polfigur berechnet. Die Auflösung der vollständigen interpolierten Polfigur wurde mit jeweils $3^{\circ}$ für die Winkel $\alpha$ und $\beta$ festgelegt. Diese interpolierte vollständige Polfigur wurde als Datensatz exportiert.

Mit dem Programm POL-READ (H. Klein, unveröffentlicht) wird die Datei des PoleFigureInterpolators in eine ascii-Datei umgewandelt. Diese ascii-Datei wurde in den meisten Fällen in das Programm cubtri (H. Klein, unveröffentlicht) eingegeben, welches nach der in Kapitel 2.5.2 beschriebenen harmonischen Methode die ODF und die rückgerechneten vollständigen Polfiguren berechnete. Hierbei geht das Programm cubtri von einer kubischen Kristallsymmetrie und einer triklinen Probensymmetrie aus. Bei einer 
triklinen Probensymmetrie sind in den Polfiguren der Probe und damit in ihrer Orientierungsverteilung keine Symmetrieelemente zu erkennen. Für das Programm cubtri bedeutet dies, dass das Programm keine Symmetrieparameter für die ODF-Berechnung festlegt. Für Proben mit anderer Proben- und Kristallsymmetrie wurden analog die entsprechenden Programme (H. Klein, unveröffentlicht) verwendet.
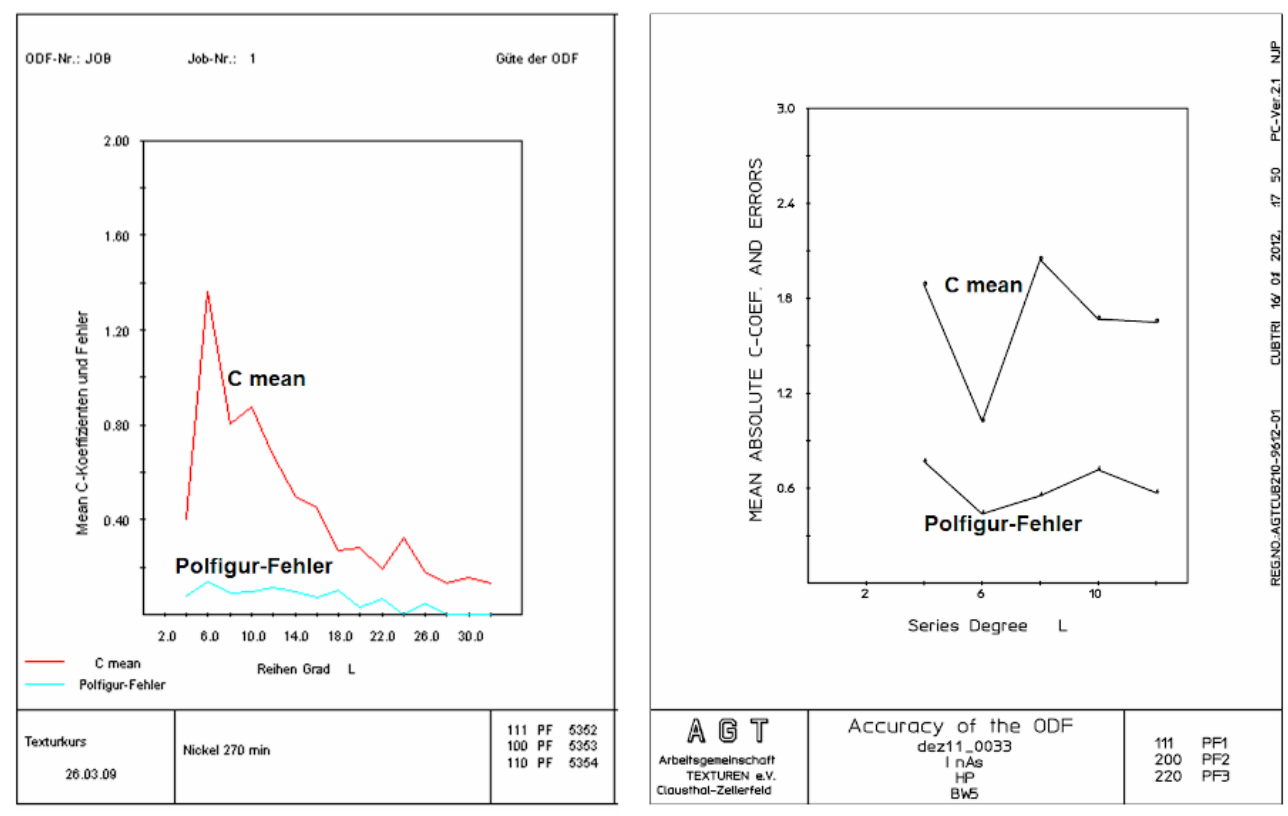

Abbildung 6.5: Konvergenz der C-Koeffizienten der Reihenentwicklung und Fehler. Links: Unter Hitze rekristallisiertes gewalztes Nickelblech (Quelle: Texturanalyse-Praktikum, H. Klein, 2009). Rechts: Hochdruckphase von InAs, Experiment dez11.

Die Überlagerung von Probenreflexen durch das Gasketmaterial und die geringe Intensität mancher Reflexe führten dazu, dass nur sehr wenige Polfiguren für die Berechnung der ODF einer Phase zur Verfügung standen, manchmal wurde sogar nur eine einzige Polfigur für eine Phase erhalten. Deshalb ließen sich keine hohen Reihenentwicklungsgrade für die ODF-Berechnung erreichen. Artefaktreflexe der Diamantstempel und die Pulverringe des Gaskets verringerten bei vielen Polfiguren trotz Untergrundkorrektur die Qualität so sehr, dass sie sich nicht sinnvoll für die ODF-Berechnung nutzen ließen. Auch führte die geringe Abdeckung des reziproken Raumes und das daraus resultierende Fehlen von Reflexen in den Polfiguren dazu, dass Orientierungen nicht richtig berechnet wurden. Abbildung 6.5 zeigt den Verlauf der C-Koeffizienten und den Fehler mit fortlaufendem Reihenentwicklungsgrad für gewalztes, rekristallisiertes Nickelblech und die Hochdruckphase des InAs-Experiments dez11. Es ist deutlich zu sehen, dass C-Koeffizienten und Fehler für die Nickelblech-Probe konvergieren, während für die InAs-Hochdruckphase 
weder für die C-Koeffizienten noch für den Fehler eine Konvergenz erhalten wurde.

So ließen sich die ODF und die berechneten vollständigen Polfiguren in einigen Fällen als Hilfsmittel zum Erkennen von Hauptorientierungen heranziehen, feine Texturdetails, die in den gemessenen Polfiguren sichtbar sind, ließen sich allerdings nicht auflösen und die berechnete Orientierungsverteilung entsprach auch nicht immer dem gemessenen Orientierungen. 


\title{
7 Ergebnisse und Interpretation
}

\begin{abstract}
Alle Experimente bis einschließlich Mai 2011 wurden von Dr. rer. nat. Heidrun Sowa und Dr. -Ing. Helmut Klein durchgeführt, die späteren Messungen unter Beteiligung der Autorin der vorliegenden Arbeit. Die einzelnen Experimente sind mit dem Zeitpunkt ihrer Durchführung bezeichnet, so trägt zum Beispiel das im August 2007 durchgeführte Experiment die Bezeichnung aug07. Die Auswertung der Experimentdaten wurde von der Autorin der vorliegenden Arbeit vorgenommen.

Im Folgenden bezeichnet die Abkürzung EK den Ausgangseinkristall, HP bezeichnet die Hochdruckphase nach dem Phasenübergang, HT die Hochtemperaturphase nach dem Phasenübergang, LP die entlastete Niederdruckphase und LT die abgekühlte Niedertemperaturphase. Der Strukturtyp der jeweiligen Phase wird bei den einzelnen Richtungen und Netzebenen als Index wie folgt angegeben: $Z n S$ steht als Kürzel für den ZinkblendeTyp, $N a C l$ für den NaCl-Typ, $W z$ für den Wurztit-Typ, $C h p$ für den Chalkopyrit-Typ, Millerit für den Millerit-Typ und NiAs für den NiAs-Typ.
\end{abstract}

\subsection{Indiumarsenid $(\operatorname{In} A s)$}

Die bisher einzige veröffentlichte experimentelle Arbeit zu Orientierungsbeziehungen zwischen Niederdruck- und Hochdruckphase von Indiumarsenid stammt von Sowa und Klein [76] und kann als Vorarbeit zur vorliegenden Arbeit aufgefasst werden. Die dort aufgeführte Messung entspricht dem Experiment sept09. Die Auswertung der Detektorbilder ergab die Orientierungsbeziehung $\langle 111\rangle_{Z n S} \|\langle 100\rangle_{N a C l}$ für eine ausgezeichnete $\langle 100\rangle_{\mathrm{NaCl}}$-Richtung.

Am Material Indiumarsenid wurden insgesamt fünf Experimente durchgeführt: 
- An der Probe aug07 wurde eine Orientierungsmessung des Einkristalls in der Las1Zelle auf Röntgenfilm durchgeführt. Anschließend wurde die Hochdruckphase bei einem Druck von 8.0 GPa an der Beamline BW5 mit dem mar345-Flächendetektor gemessen. Hierbei wurde ein Winkelbereich $-36^{\circ} \leq \omega \leq 36^{\circ}$ abgedeckt, mit einer Schrittweite von $\Delta \omega=2^{\circ}$. Die Wellenlänge betrug $\lambda=0.11944 \AA$ und der ProbeDetektor-Abstand $1255 \mathrm{~mm}$.

- Für die Probe sept09 existiert keine Einkristallmessung. Die Hochdruckphase der Probe wurde in der runden Vierschraubenzelle bei einem Druck von 7.0 GPa, die entlastete Niederdruckphase bei einem Druck von 3.1 GPa an der Beamline BW5 mit dem mar345-Flächendetektor gemessen. Es wurde ein $\omega$-Bereich von $-30^{\circ} \leq \omega \leq 30^{\circ}$, mit $\Delta \omega=1^{\circ}$ untersucht. Die Wellenlänge lag bei $\lambda=0.1255 \AA$, der Probe-Detektor-Abstand betrug 1440 mm. Die Messung der Hochdruckphase ist nicht sehr aussagekräftig.

- Die Messungen der Probe mai10juli10 wurden an der Beamline BW5 in der Las1Zelle durchgeführt. Als Detektor kam der mar345 Flächendetektor zum Einsatz. Der abgedeckte Winkelbereich betrug $-42^{\circ} \leq \omega \leq 42^{\circ}$, und $\Delta \omega=1^{\circ}$. Der Einkristall bei einem Druck von 6.5 GPa wurde mit einer Wellenlänge von $\lambda=0.13112 \AA$ und einem Probe-Detektor-Abstand von $1192 \mathrm{~mm}$ gemessen. Die Hochdruckphase bei einem Druck von 9.2 GPa und die auf 2.5 GPa entlastete Niederdruckphase wurden mit einer Wellenlänge von $\lambda=0.1614 \AA$ und einem Probe-DetektorAbstand von 1366 mm gemessen. Von den Messungen ließ sich nur die Hochdruckphase für eine Auswertung verwenden.

- Das Experiment mit der Probe dez11 wurde in der Las1-Zelle durchgeführt. Die Messungen fanden an der Beamline BW5 statt und es wurde ein Perkin Elmer 1621Detektor verwendet. Zuerst wurde der Einkristall bei einem Druck von 5.8 GPa mit Schritten von $\Delta \omega=0.2^{\circ}$ gemessen, anschließend wurde der Druck auf 7.6 GPa erhöht, wobei sich das Material teilweise in seine Hochdruckphase umwandelte. Die Schrittweite dieser Messung betrug $\Delta \omega=0.4^{\circ}$. Bei einem Druck von 3.8 GPa setzte eine Rückumwandlung in die Niederdruckphase ein, die bei 3.0 GPa abgeschlossen war. Auch diese Modifikation wurde mit einer Schrittweite von $\Delta \omega=$ $0.4^{\circ}$ gemessen. Der abgedeckte Bereich betrug bei allen Messungen $-42^{\circ} \leq \omega \leq$ $42^{\circ}$, bei einer Wellenlänge $\lambda=0.12399 \AA$ und einem Probe-Detektor-Abstand von $1468 \mathrm{~mm}$. Im Verlauf der Druckentlastung verrutschte der Kristall leicht in der 
Probenkammer, was sich jedoch bei der Auswertung aufgrund der Orientierung der jeweiligen Phasenreste bei den einzelnen Messungen korrigieren ließ.

- Die Messungen der Probe okt13 wurden an der Beamline P02.1 in der Las1-Zelle durchgeführt. Als Detektor kam das Perkin Elmer 1621-Modell zum Einsatz. Es wurden die Hochdruckphase bei einem Druck von 7.7 GPa und die entlastete Niederdruckphase bei 2.9 GPa gemessen. Der abgedeckte Winkelbereich betrug $-42^{\circ} \leq \omega \leq 42^{\circ}$, und $\Delta \omega=1^{\circ}$. Die verwendete Wellenlänge war $\lambda=0.207 \AA$ und der Probe-Detektor-Abstand $800 \mathrm{~mm}$.
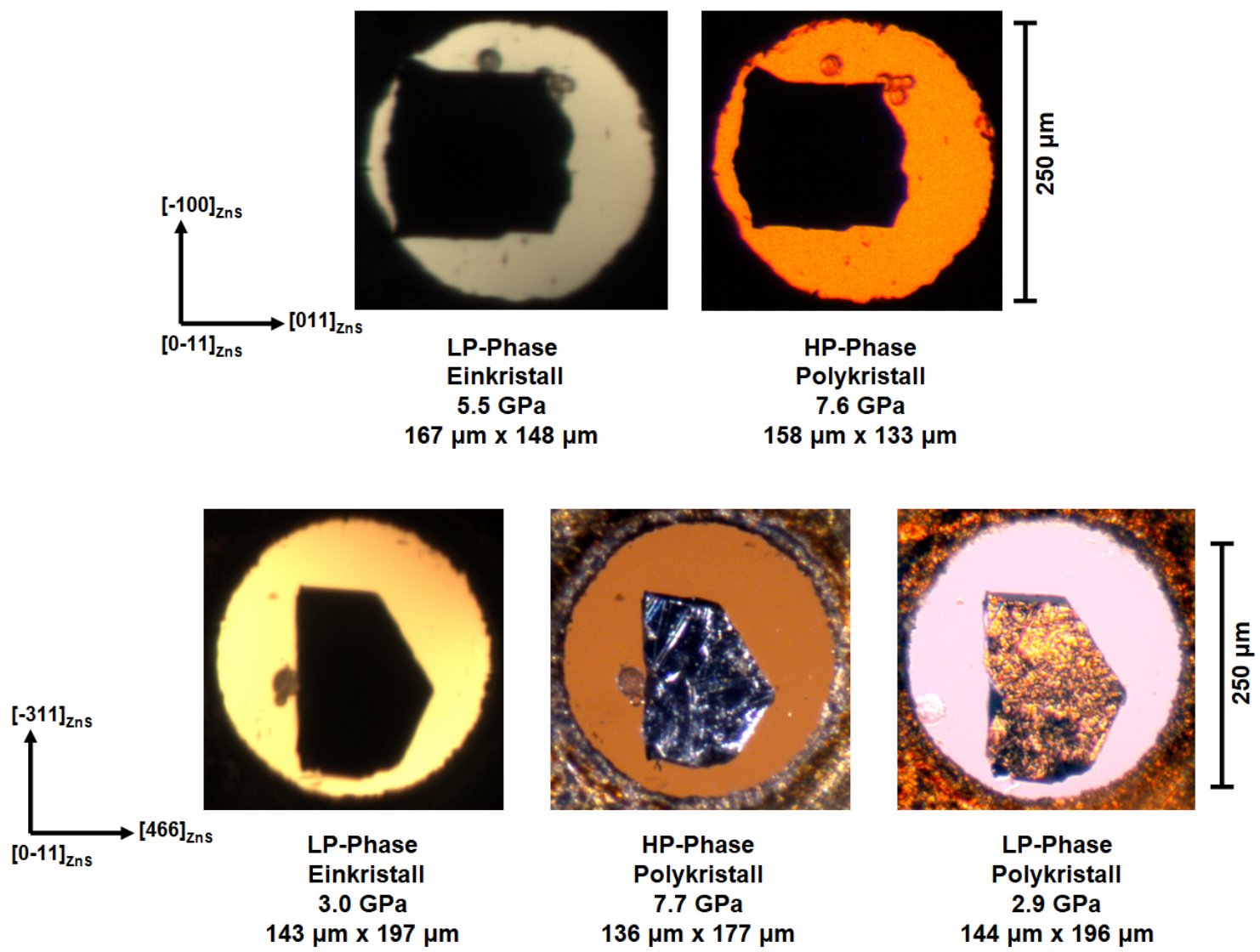

Abbildung 7.1: Aufnahmen der InAs-Proben des Experimente dez11 (obere Reihe) und okt13 (untere Reihe). Das Koordinatensystem auf der linken Seite zeigt die jeweilige grobe Orientierung des Einkristalls zu Versuchsbeginn an.

Bei der Probe dez11 wurde während der Phasenumwandlung eine Schrumpfung um 10\% in der Längsrichtung $[001]_{Z n S}$ des Einkristalls und um 5\% in Querrichtung $[110]_{Z n S}$ festgestellt. Für die Probe okt13 besaßen Längs- und Querrichtung in der Ausgangsorientierung des Einkristalls keine niedrigen Indizes, die angegebenen Richtungen sind Näherungen. Hier betrug die Schrumpfung in Längsrichtung 10\% und in Querrichtung 
5\%. Abbildung 7.1 zeigt die dazugehörigen Mikroskopaufnahmen. Die Normalenrichtung der Einkristall-Ausgangsorientierung ist bei beiden Proben eine $\langle 110\rangle_{Z n S}$-Richtung, aufgrund der Druckzellengeometrie konnte die Schrumpfung nicht gemessen werden.

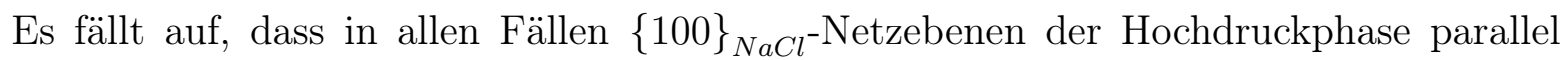
zu $\{111\}_{Z n S^{-}}$Netzebenen der Niederdruckphase liegen (siehe Abbildung 7.2). Da alle Hauptrichtungen im kubischen Kristallsystem symmetrisch äquivalent sind, wurde die durch diese Symmetriebeziehung ausgezeichnete Richtung für die Auswertung als $[001]_{\mathrm{NaCl}}$-Richtung definiert. Die übrigen $\{100\}_{\mathrm{NaCl}^{-}}$Pole bilden im $90^{\circ}$-Winkel dazu im gesamten messbaren Polfigurbereich einen Gürtel mit Intensitätsmaxima in $15^{\circ}$ Abständen. Diese Maxima sind viel schwächer als die $(001)_{N a C l}$-Pole. Sie weisen innerhalb jedes Gürtels etwa gleiche Intensitäten auf, was darauf schließen lässt, dass keine Gürtelposition bevorzugt wird. Diese Orientierungen wurden bei allen InAs-Experimenten beobachtet (Abbildung 7.3) Entsprechend liegen auch die $\{110\}_{\mathrm{NaCl}^{-}}$und die $\{111\}_{\mathrm{NaCl}^{-}}$ Pole der Hochdruckphase auf Gürteln mit Maxima im Abstand von 15², wobei einer der Gürtel der $\{110\}_{N a C l}$-Pole zum Gürtel der $\{100\}_{\mathrm{NaCl}}$-Pole deckungsgleich ist (siehe Abbildung 7.4).

Hiermit gilt für alle möglichen Orientierungen die Orientierungsbeziehung

$$
\langle 111\rangle_{Z n S} \|[001]_{N a C l}
$$

mit ihrem Äquivalent in der Gegenrichtung

$$
\langle\overline{1} \overline{1} \overline{1}\rangle_{Z n S} \|[00 \overline{1}]_{N a C l}
$$

Die entlastete Niederdruckphase weist nur eine einzige erkennbare Orienterung auf. Sie entspricht der Orientierung des Ausgangseinkristalls.

Abbildung 7.5 zeigt die aus den berechneten F-Koeffizienten berechneten vollständigen Polfiguren der $\{100\}_{N a C l}$-Flächenpole der Hochdruckphase und der $\{111\}_{Z n S}$-Flächenpole der Niederdruckphase für das InAs-Experiment dez11. Die Orientierung der berechneten Polfiguren entspricht der Orientierung der gemessenen Polfiguren. Es ist zu erkennen, dass es möglich ist, die Hauptorientierungen der $\{100\}_{\mathrm{NaCl}}$-Flächenpole und die Lage der Orientierungsgürtel richtig zu bestimmen, die Auflösung der einzelnen Orientierungen in 

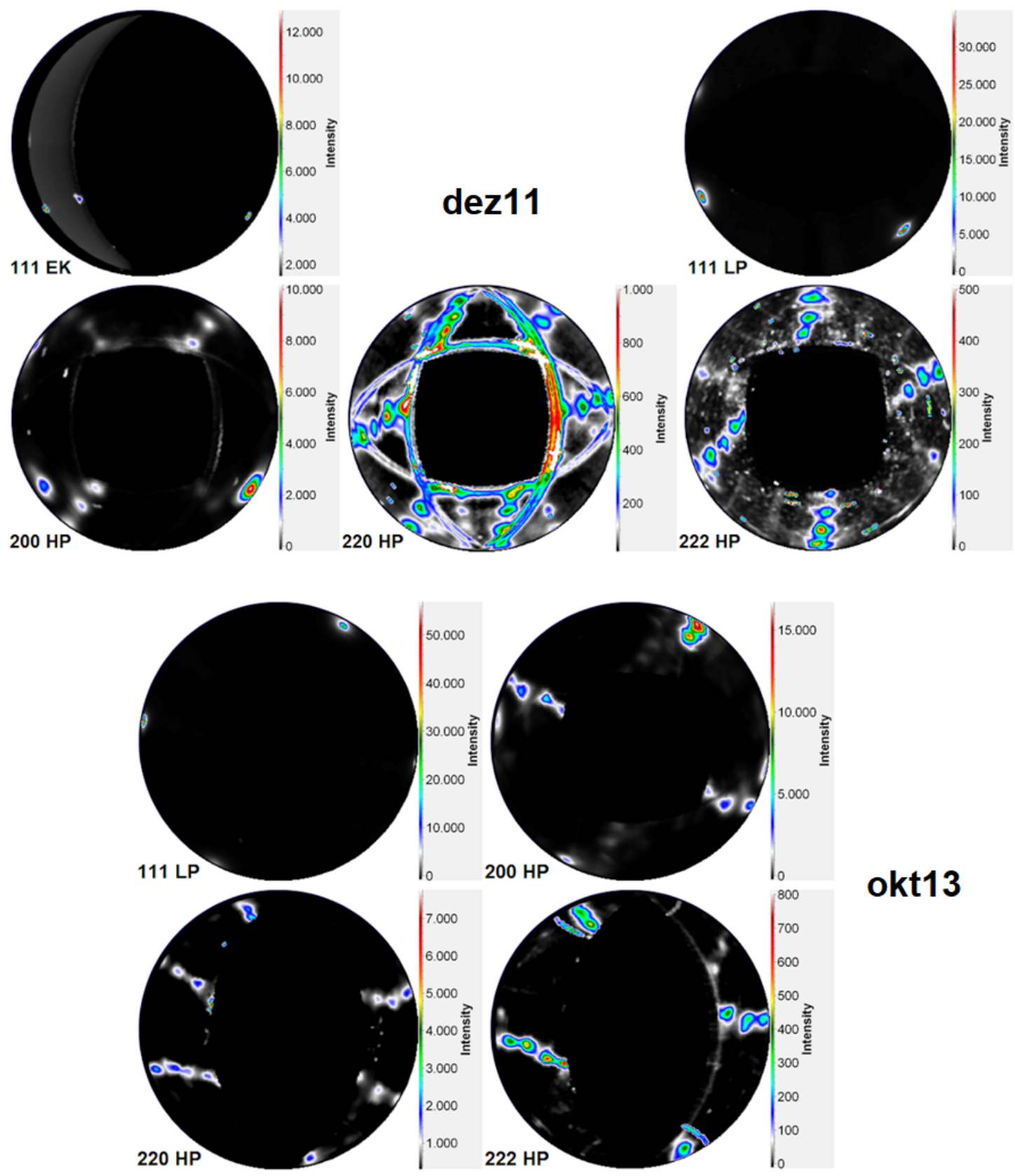

\section{okt13}

Abbildung 7.2: Ausgewählte Polfiguren der InAs-Experimente dez11 und okt13. Es ist deutlich zu sehen, dass die sichtbaren Flächenpole der $\{111\}_{Z n S}$ Netzebenen auf Flächenpolen der $\{100\}_{\mathrm{NaCl}}$-Netzebenen liegen. Ebenso ist der Gürtel mit den in $15^{\circ}$-Abständen auftretenden weiteren $\{100\}_{\mathrm{NaCl}^{-N}}$-Netzebenenorientierungen zu sehen. Die $\{110\}_{\mathrm{NaCl}^{-}}$ Polfiguren der Hochdruckphase zeigen, dass die $\{110\}_{\mathrm{NaCl}}$-Flächenpole auf drei Gürteln liegen. Es ist zu sehen, dass die $\{110\}_{\mathrm{NaCl}}$-Flächenpole des mittleren Gürtels auf den gleichen Stellen wie die $\{100\}_{\mathrm{NaCl}}$-Flächenpole liegen. Die $\{111\}_{\mathrm{NaCl}}$-Polfiguren der Hochdruckphase zeigen die zwei Orientierungsgürtel der $\{111\}_{\mathrm{NaCl}}$-Flächenpole. 


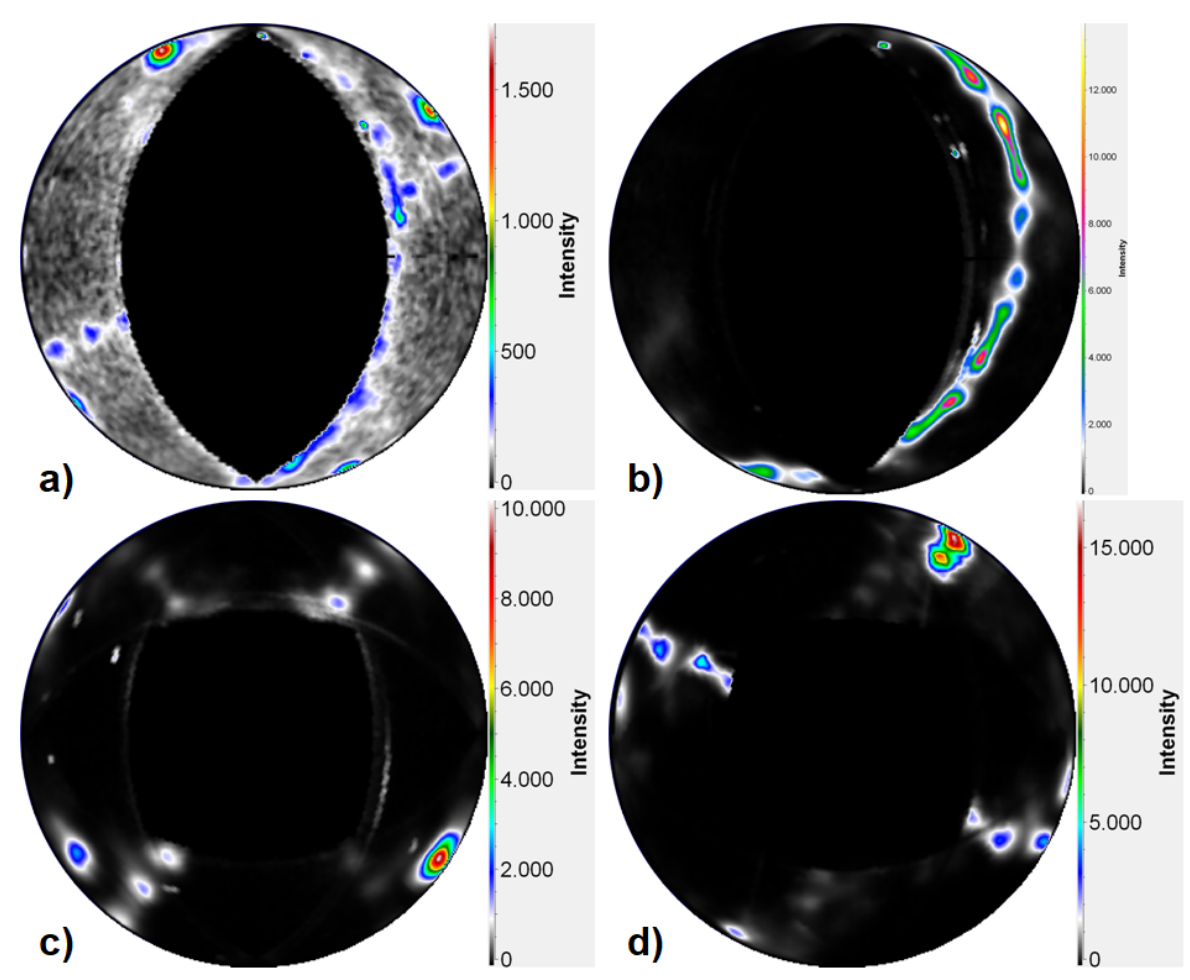

Abbildung 7.3: $\{100\}_{N_{a C l}}$-Polfiguren der Hochdruckphase der InAs-Proben a) aug07, b) mai10juli10, c) dez11, d) okt13. Auf allen Polfiguren ist der Orientierungsgürtel der $\{100\}_{\mathrm{NaCl}^{-}}$ Flächenpole senkrecht zum dazugehörigen $(001)_{N a C l}$-Flächenpol zu sehen. Ebenso wird deutlich, dass nicht alle denkbaren $\langle 111\rangle_{Z_{n S}} \|[001]_{N a C l}$-Orientierungen in gleicher Intensität auftreten, manche sogar gar nicht. Das Experiment aug07 zeigt zwei Orientierungen ähnlicher Intensität, wobei bei einer der Gürtel nur zu erahnen ist, von einer dritten Orientierung ist nur der Gürtel zu sehen, da der dazugehörige (001) $\mathrm{NaCl}_{\text {-Flächenpol }}$ außerhalb des Messbereichs der Druckzelle liegt. Das Experiment mai10juli10 zeigt lediglich den Gürtel einer Orientierung, der dazugehörige (001) ${ }_{\mathrm{NaCl}}$-Flächenpol konnte aufgrund der Druckzellengeometrie nicht erfasst werden. Im Experiment dez11 sind zwei Orientierungen zu sehen, wobei eine nur untergeordnet auftritt. Die gleiche Beobachtung wurde für das Experiment okt13 gemacht. 


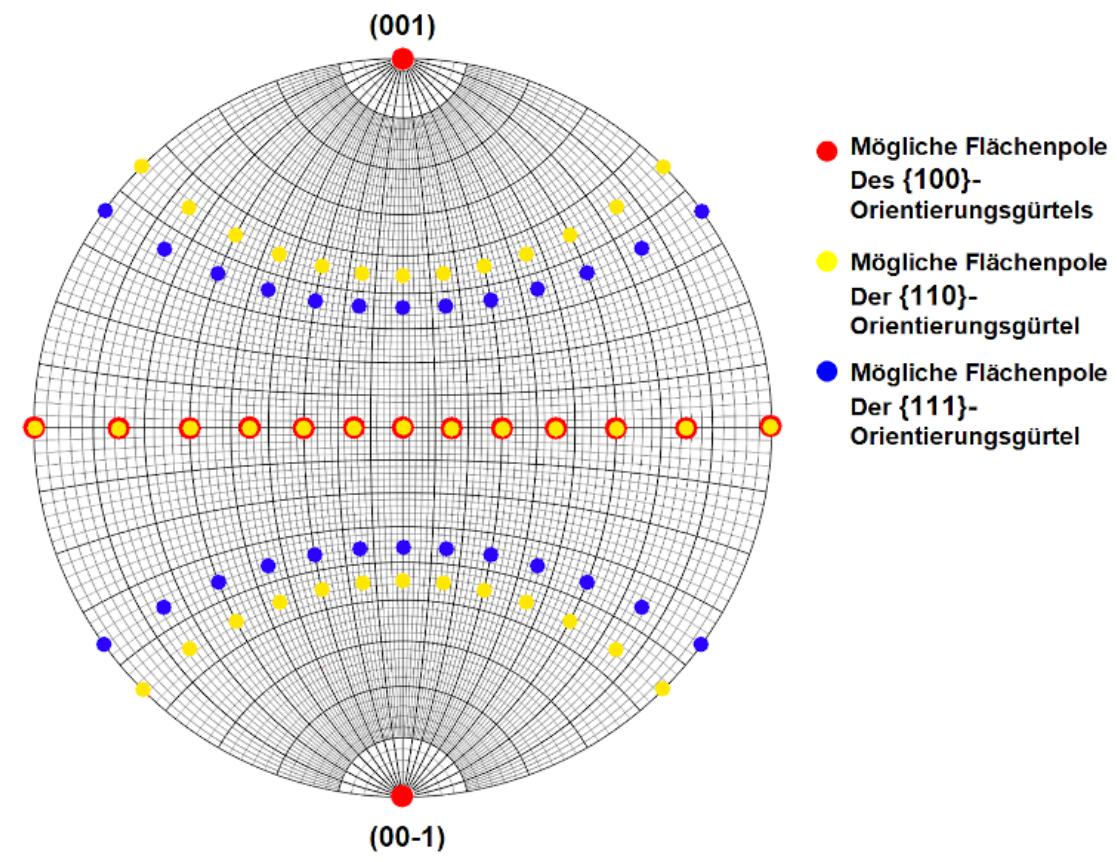

Abbildung 7.4: Schema aller Orientierungsgürtel der $\{100\}$-, $\{110\}$ - und $\{111\}$-Flächenpole für einen gegebenen (001)-Flächenpol der Hochdruckphase.

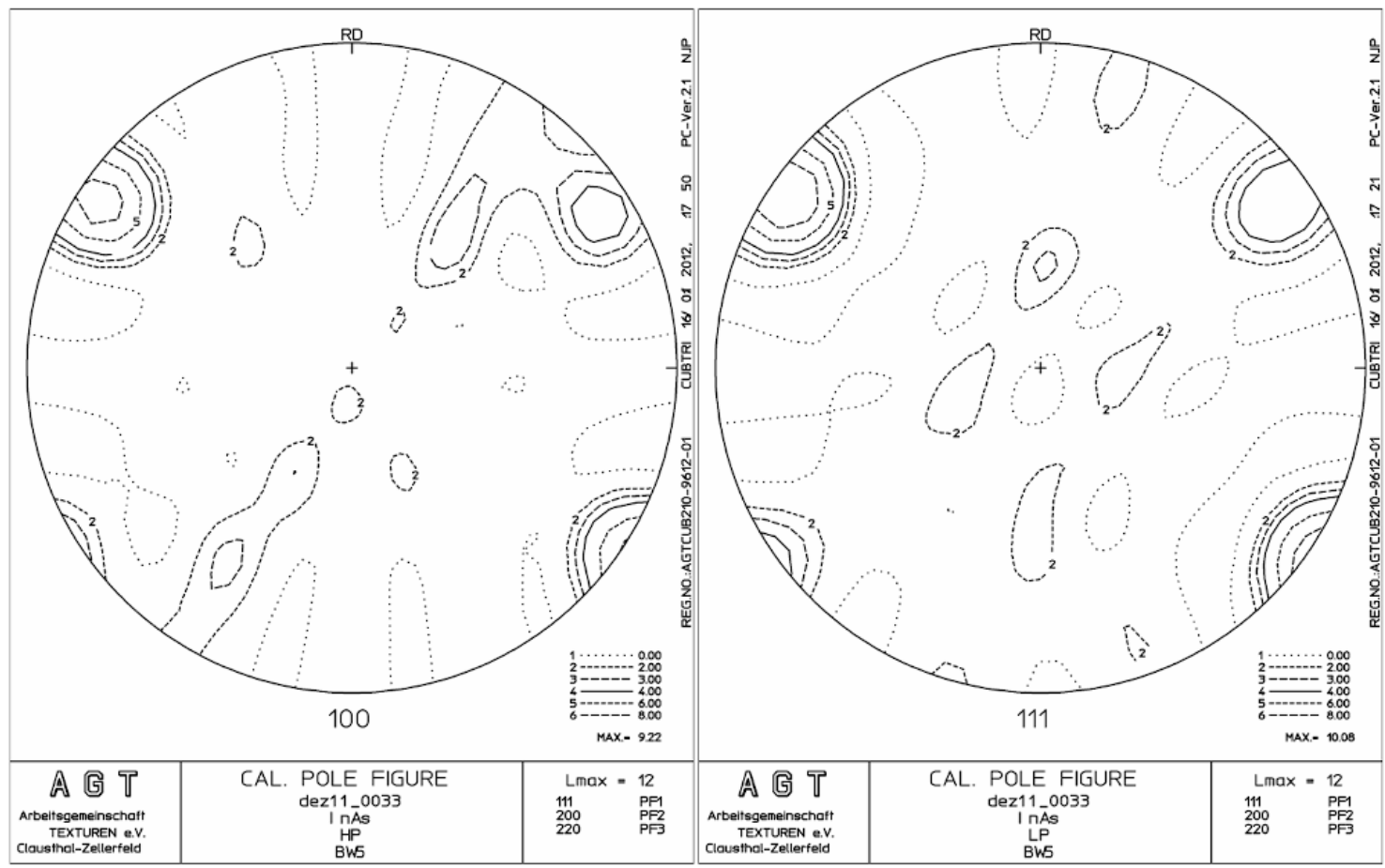

Abbildung 7.5: Rückgerechnete vollständige Polfiguren des InAs-Experiments dez11. Links die $\{100\}$ Flächenpole der Hochdruckphase, rechts die $\{111\}$-Flächenpole der Niederdruckphase. 
$15^{\circ}$-Schritten in den Orientierungsgürteln war aufgrund der niedrigen Intensitäten und des großen fehlenden Bereichs in den gemessenen Polfiguren nicht möglich. Die Polfigur der $\{111\}_{Z n S}$-Flächenpole zeigt alle gemessenen Pole, allerdings wären bei der vollständigen Polfigur zwei weitere $\{111\}_{Z n S^{-}}$Pole gleicher Intensität bei $\alpha=36^{\circ}$ und $\beta=90^{\circ}$ bzw. $270^{\circ}$ erwartet worden. Stattdessen sind nur niedrige Intensitäten zu sehen, die kaum aus dem Untergrund hervorstechen.

a)

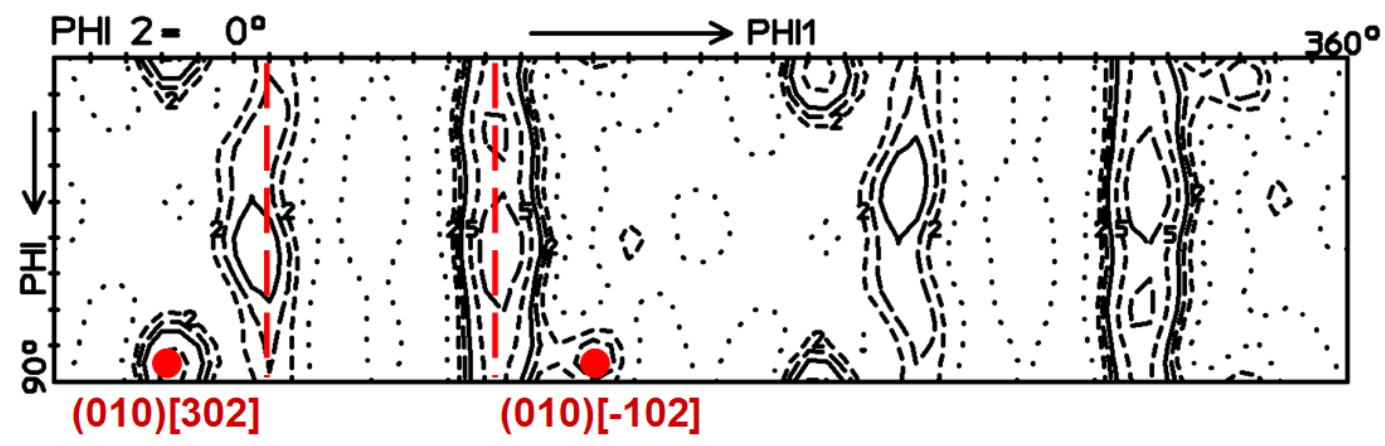

b)

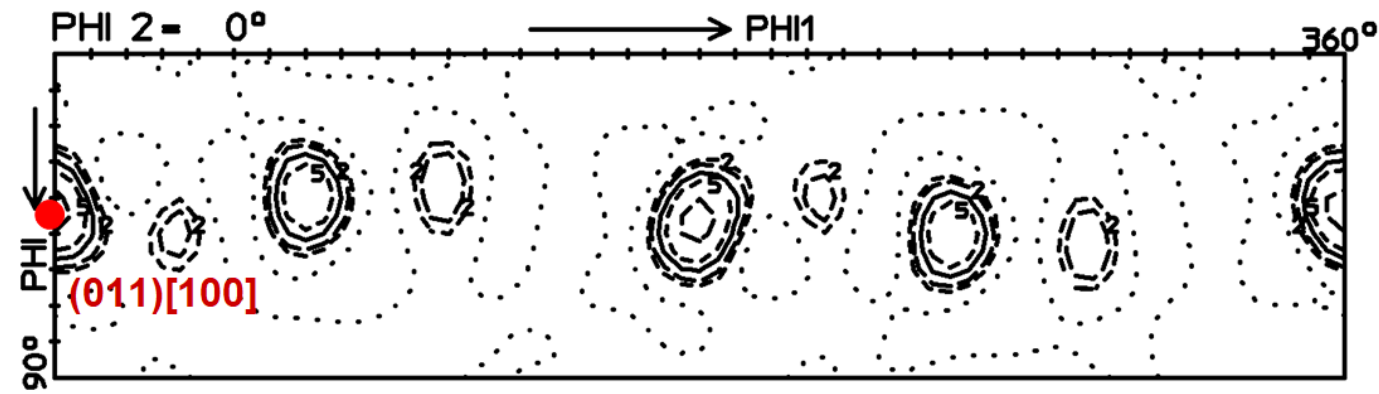

Abbildung 7.6: Berechnete ODF des InAs-Experiments dez11 für den Euleraumschnitt $\varphi_{2}=0$. a) Hochdruckphase. b) entlastete Niederdruckphase mit Reihenentwicklungsgrad $L_{\max }=12$. Die nicht indizierten Lagen sind symmetrisch gleichwertig mit den indizierten Lagen.

Der $\varphi_{2}=0^{\circ}$-Schnitt der ODF für die Hochdruckphase des InAs-Experiments dez11 (Abbildung 7.6) weist zwei Einzelorientierungen (010) [302] und (010) [102] auf, die den beiden gemessenen und auch in der berechneten $\{100\}$-Polfigur sichtbaren Hauptorientierungen entsprechen. Zwei nicht ideale $\Phi$-Fasern sind bei $\varphi_{1}=60^{\circ}$ und $\varphi_{1}=123^{\circ} \mathrm{zu}$ finden. Diese beiden Fasern entsprechen den Orientierungsgürteln senkrecht zur Hauptorientierung und liegen somit um $90^{\circ}$ in $\varphi_{1}$ gegen ihre zugehörige Hauptorientierung versetzt. Die in den gemessenen Polfiguren beobachteten Maxima in $15^{\circ}$-Abständen konnten durch die ODF-Berechnung nicht aufgelöst werden.

Der $\varphi_{2}=0^{\circ}$-Schnitt der ODF für die Niederdruckphase des InAs-Experiments dez11 (Abbildung 7.6) weist zusätzlich zur tatsächlichen Probenorientierung (011) [100] noch weitere Orientierungen auf, die keine Entsprechung in den gemessenen Polfiguren haben. 
Es wird vermutet, dass durch das Fehlen zweier $\{111\}_{Z n S}$-Flächenpole in der berechneten Polfigur die Orientierungsverteilung fälschlicherweise von dem Programm cubtri als mehrere Einzelorientierungen berechnet wurde. Dafür spricht der Winkel von ca. $\varphi_{1}=70^{\circ}$ zwischen den Orientierungen, der dem kleineren Winkel zwischen zwei $\{111\}_{Z n S^{-}}$ Flächenpolen in der Polfigur entspricht. Weitere, wesentlich schwächere Orientierungen entstammen dem Untergrund. Die ODF wurde mit einem Reihenentwicklungsgrad $L_{\max }=12$ berechnet. Ein höherer Reihenentwicklungsgrad führte zu schärferen Orientierungen und einem niedrigeren Untergrund, ließ aber auch den schwächeren Orientierungsgürtel der Hochdruckphase verschwinden. Abbildung 10.2 und 10.3 zeigen alle berechneten Eulerraumschnitte des InAs-Experiments dez11. Der $\varphi_{2}=0^{\circ}$-Schnitt wurde für die Demonstration gewählt, weil dort in der Hochdruckphase die $\Phi$-Fasern am deutlichsten zu erkennen sind. Die nicht beschrifteten Lagen sind symmetrisch zu den beschrifteten Lagen.

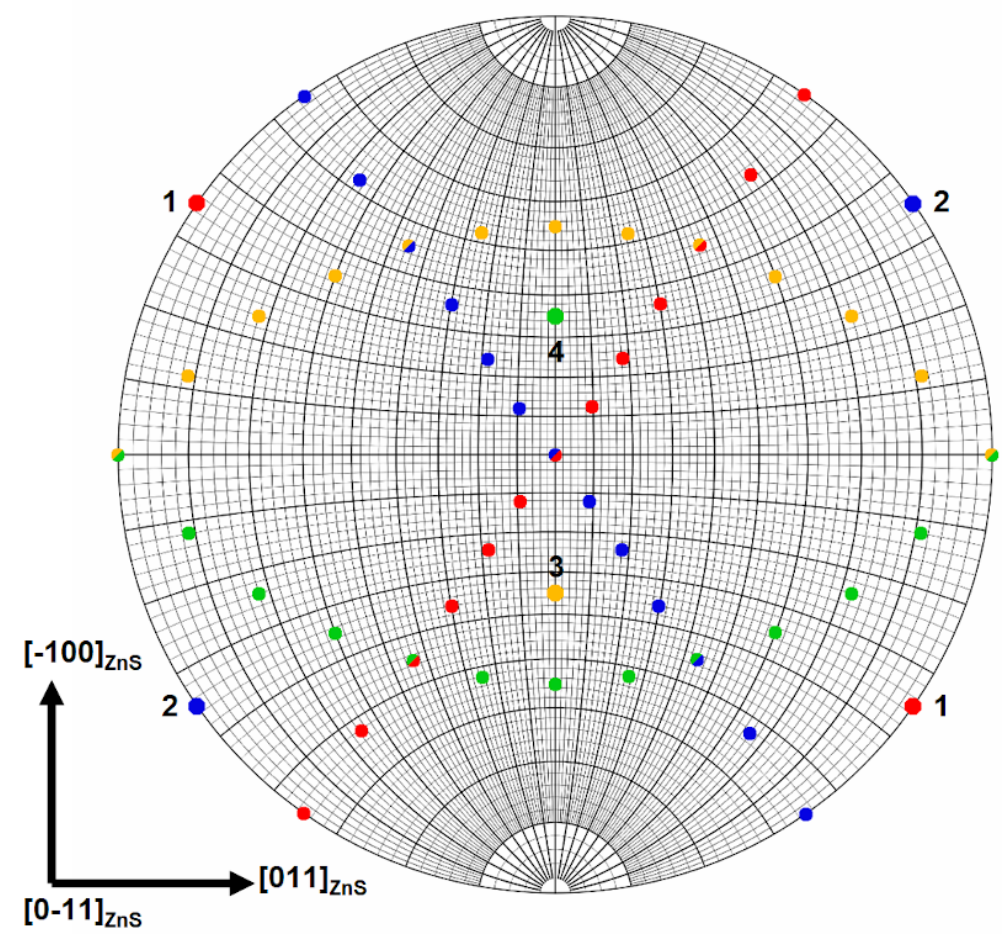

Abbildung 7.7: Schematische Darstellung der Lagen der $\{100\}_{\mathrm{NaCl}}$-Pole der Hochdruckphase von InAs. Eine Farbe stellt jeweils alle Pole für eine Orientierung mit $[001]_{\mathrm{NaCl}} \|(111)_{Z n S}$ dar. Diese Hauptrichtungen sind duchnummeriert und liegen an der gleichen Stelle wie die $\{111\}_{Z n S}$-Pole der Niederdruckphase.

Bei der Umwandlung des Einkristalls der Niederdruckphase ergeben sich vier mögliche Orientierungen für die $[001]_{\mathrm{NaCl}}$-Richtung der Hochdruckphase (beziehungsweise acht, 
falls zwischen $[u v w]$ und $[\bar{u} \bar{v} \bar{w}]$ unterschieden wird), wobei die Elementarzelle in $15^{\circ}$ Intervallen um diese rotiert werden kann. Abbildung 7.7 zeigt sämtliche möglichen Positionen der $\{100\}_{\mathrm{NaCl}}$-Pole der Hochdruckphase im Wulffschen Netz. Im Experiment traten niemals alle möglichen Orientierungen auf. Es wurde beobachtet, dass eine der $\langle 111\rangle_{Z n S^{-}}$-Richtungen der Niederdruckphase bevorzugt zur $[001]_{\mathrm{NaCl}^{-R i c h t u n g}}$ der Hochdruckphase wird. Weitere Orientierungen $\langle 111\rangle_{Z n S} \|[001]_{N a C l}$ treten nur untergeordnet oder gar nicht auf (siehe Abbildung 7.3).
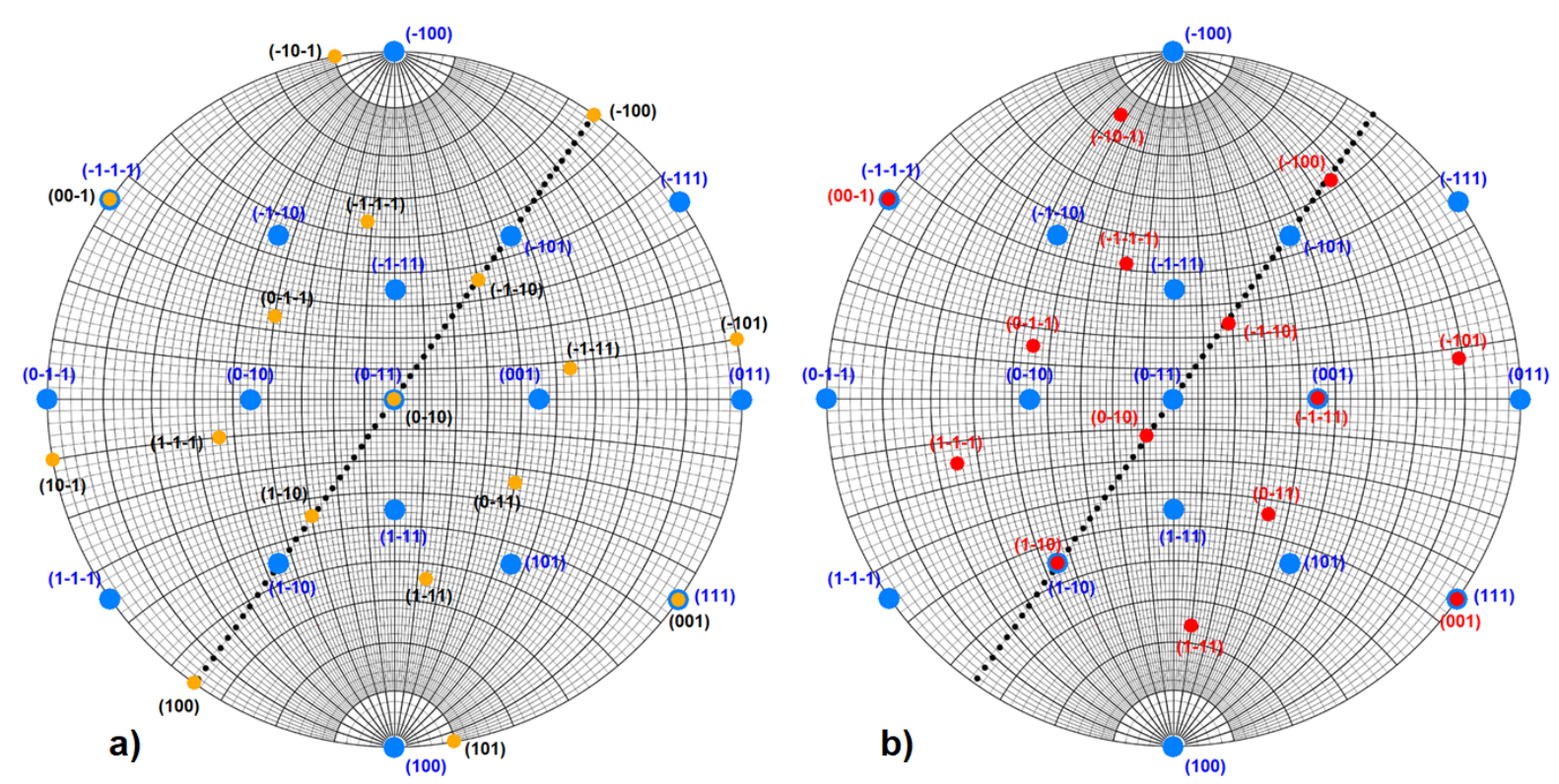

Abbildung 7.8: Beispiele für die zwei möglichen Versionen von Orientierungsbeziehungen zwischen Niederdruck- und Hochdruckphase von InAs, basierend auf Hauptorientierung 1 aus Abbildung 7.7. Aus Gründen der Übersichtlichkeit ist je nur eine Orientierung dargestellt. Die blauen Punkte bezeichnen die Flächenpole der Niederdruckphase. a) Die gelben Punkte stellen die Flächenpole einer $\langle 110\rangle_{Z n S} \|\langle 100\rangle_{N a C l}$-Orientierung dar. b) Die roten Punkte stellen die Flächenpole einer $\langle 110\rangle_{Z n S} \|\langle 110\rangle_{N a C l}$-Orientierung dar. Die schwarze Linie kennzeichnet den Gürtel auf dem die $\{100\}_{\mathrm{NaCl}^{-}}$und $\{110\}_{\mathrm{NaCl}}$-Pole der Hochdruckphase liegen. Es ist nur die obere Hälfte der Lagenkugel dargestellt.

Für die folgenden Betrachtungen wird der Einfachheit halber lediglich auf die Orientierung $[111]_{Z n S} \|[001]_{N a C l}$ Bezug genommen, jedoch gelten die Beobachtungen genauso entsprechend für die übrigen Orientierungen der Hochdruckphase.

Auf dem um $90^{\circ}$ zur Hauptrichtung gelegenen Orientierungsgürtel der Hochdruckphase liegen sechs der insgesamt $12\langle 110\rangle$-Richtungen der Niederdruckphase. Dies sind die

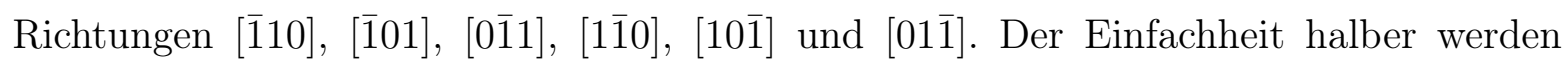
sie im folgenden als $\langle 110\rangle_{Z n S}$ zusammengefasst. Aufgrund der $15^{\circ}$-Reflexabstände der $\{100\}_{\mathrm{NaCl}}$-Pole als auch der $\{110\}_{\mathrm{NaCl}^{-}}$-Pole entlang des Gürtels und ihrer Deckungs- 
gleichheit gibt es zwei Möglichkeiten für Orientierungsbeziehungen.

Orientierungsmöglichkeit 1 (Abbildung 7.8a) tritt ein, wenn eine der sich auf dem Gürtel befindenden $\langle 100\rangle_{\mathrm{NaCl}}$-Richtungen der Hochdruckphase parallel zu einer der genannten $\langle 110\rangle_{Z n S}$-Richtungen der Niederdruckphase befindet:

$$
[100]_{N a C l} \vee[010]_{N a C l} \vee[\overline{1} 00]_{N a C l} \vee[0 \overline{1} 0]_{N a C l} \|\langle 110\rangle_{Z n S}
$$

Im Folgenden werden die in Gleichung 7.3 genannten vier Richtungen der $\langle 100\rangle$-Familie als $\langle 100\rangle_{\mathrm{NaCl}}$ zusammengefasst.

Orientierungsmöglichkeit 2 (Abbildung 7.8b) ist immer um $15^{\circ}$ gegenüber Orientierungsmöglichkeit 1 versetzt. Sie tritt auf, wenn eine der vier auf dem Gürtel liegenden $\langle 110\rangle_{\mathrm{NaCl}^{-}}$-Richtungen der Hochdruckphase sich parallel zu einer der genannten $\langle 110\rangle_{Z n S^{-}}$ Richtungen der Niederdruckphase befindet.

$$
[110]_{N a C l} \vee[\overline{1} 10]_{N a C l} \vee[1 \overline{1} 0]_{N a C l} \vee[\overline{1} \overline{1} 0]_{N a C l} \|\langle 110\rangle_{Z n S}
$$

Im Folgenden werden die in 7.4 genannten vier Richtungen der $\langle 110\rangle$-Familie als $\langle 110\rangle_{\mathrm{NaCl}}$ zusammengefasst.

Eine weitere Orientierungsbeziehung, die ausschließlich gemeinsam mit Orientierungsmöglichkeit 2 auftritt, ist die Beziehung

$$
[111]_{N a C l} \vee[\overline{1} 11]_{N a C l} \vee[1 \overline{1} 1]_{N a C l} \vee[\overline{1} \overline{1} 1]_{N a C l} \|[001]_{Z n S}
$$

beziehungsweise

$$
[11 \overline{1}]_{N a C l} \vee[\overline{1} 1 \overline{1}]_{N a C l} \vee[1 \overline{1} \overline{1}]_{N a C l} \vee[\overline{1} \overline{1} \overline{1}]_{N a C l} \|[00 \overline{1}]_{Z n S}
$$

Sie tritt nur in einer von drei möglichen Varianten von Orientierungsmöglichkeit 2, d. h. alle $90^{\circ}$, auf. Hierfür muss die Bedingung erfüllt sein, dass eine der $\langle 110\rangle_{N a C l}$ parallel zu $[\overline{1} 10]_{Z n S}$ bzw. $[1 \overline{1} 0]_{Z n S}$ liegt.

In Abbildung 7.8 sind beide Orientierungsmöglichkeiten beispielhaft dargestellt. Aus dem dargestellten Beispiel ergibt sich für Orientierungsmöglichkeit $\left(\langle 110\rangle_{\mathrm{ZnS}} \|\langle 100\rangle_{\mathrm{NaCl}}\right)$ 
folgende Achsentransformation:

$$
\begin{array}{r}
\vec{a}_{N a C l}=-2 \vec{a}_{Z n S}+\vec{b}_{Z n S}+\vec{c}_{Z n S} \\
\vec{b}_{N a C l}=\vec{b}_{Z n S}-\vec{c}_{Z n S} \\
\vec{c}_{N a C l}=\vec{a}_{Z n S}+\vec{b}_{Z n S}+\vec{c}_{Z n S}
\end{array}
$$

mit der Transformationsmatrix

$$
P=\left(\begin{array}{ccc}
-2 & 0 & 1 \\
1 & 1 & 1 \\
1 & -1 & 1
\end{array}\right)
$$

Die möglichen Atomverschiebungen, die zu den erhaltenen Orientierungsbeziehungen führen, sind in Abbildung 7.9 dargestellt. Die Darstellung ist senkrecht zur $[111]_{Z n S^{-}}$ Richtung der Niederdruckphase bzw. zur [001 $]_{\mathrm{NaCl}}$-Richtung der Hochdruckphase. Der auf der linken Seite abgebildete Mechanismus entspricht der $\langle 110\rangle_{\mathrm{ZnS}} \|\langle 110\rangle_{\mathrm{NaCl}^{-}}$Orientierung und zeigt die drei Möglichkeiten, wie sich die Atome entlang der eingezeichneten Geraden A, B und C gegeneinander verschieben können. Hieraus ergeben sich die links unten abgebildeten drei verschiedenen Orientierungen der $\langle 100\rangle_{\mathrm{NaCl}}$-Richtungen der Hochdruckphase, die um $30^{\circ}$ gegeneinander um die $[001]_{\mathrm{NaCl}}$-Richtung der Hochdruckphase verdreht sind. Der auf der rechten Seite abgebildete Mechanismus entspricht $\operatorname{der}\langle 110\rangle_{Z n S} \|\langle 100\rangle_{N a C l}$-Orientierung. Es findet eine Kompression entlang einer der drei angegebenen $\langle 110\rangle_{Z n S}$-Richtungen statt, hier mit A, B und C markiert, und einer gleichzeitigen Dehnung senkrecht hierzu. Hieraus ergeben sich die rechts unten abgebildeten drei verschiedenen Orientierungen der $\langle 100\rangle_{\mathrm{NaCl}^{-}}$-Richtungen, die um $30^{\circ}$ gegeneinander um die $[001]_{\mathrm{NaCl}}$-Richtung verdreht sind. Werden alle durch diese beiden Mechanismen erhaltenen Orientierungen zusammen betrachtet, ergibt sich ein Winkel von $15^{\circ}$ zwischen zwei benachbarten Orientierungen. Beiden Mechanismen ist gemein, dass keine Bindungen aufgebrochen werden müssen. Anhand der Intensitäten der Orientierungen im Orientierungsgürtel der Hochdruckphasen-Polfiguren gibt es keine eindeutigen Hinweise, dass einer der beiden Mechanismen bevorzugt wird. Zusätzlich zu den beschriebenen Vorgängen kommt es beim Phasenübergang nach Mechanismus $1 \mathrm{zu}$ weiteren Metrikanpassungen und bei Mechanismus 2 zu leichten Atomverschiebungen. Die Atomverschiebungen in Richtung der Normalenrichtung von Abbildung 7.9 wurden in der Abbildung nicht berücksichtigt. 

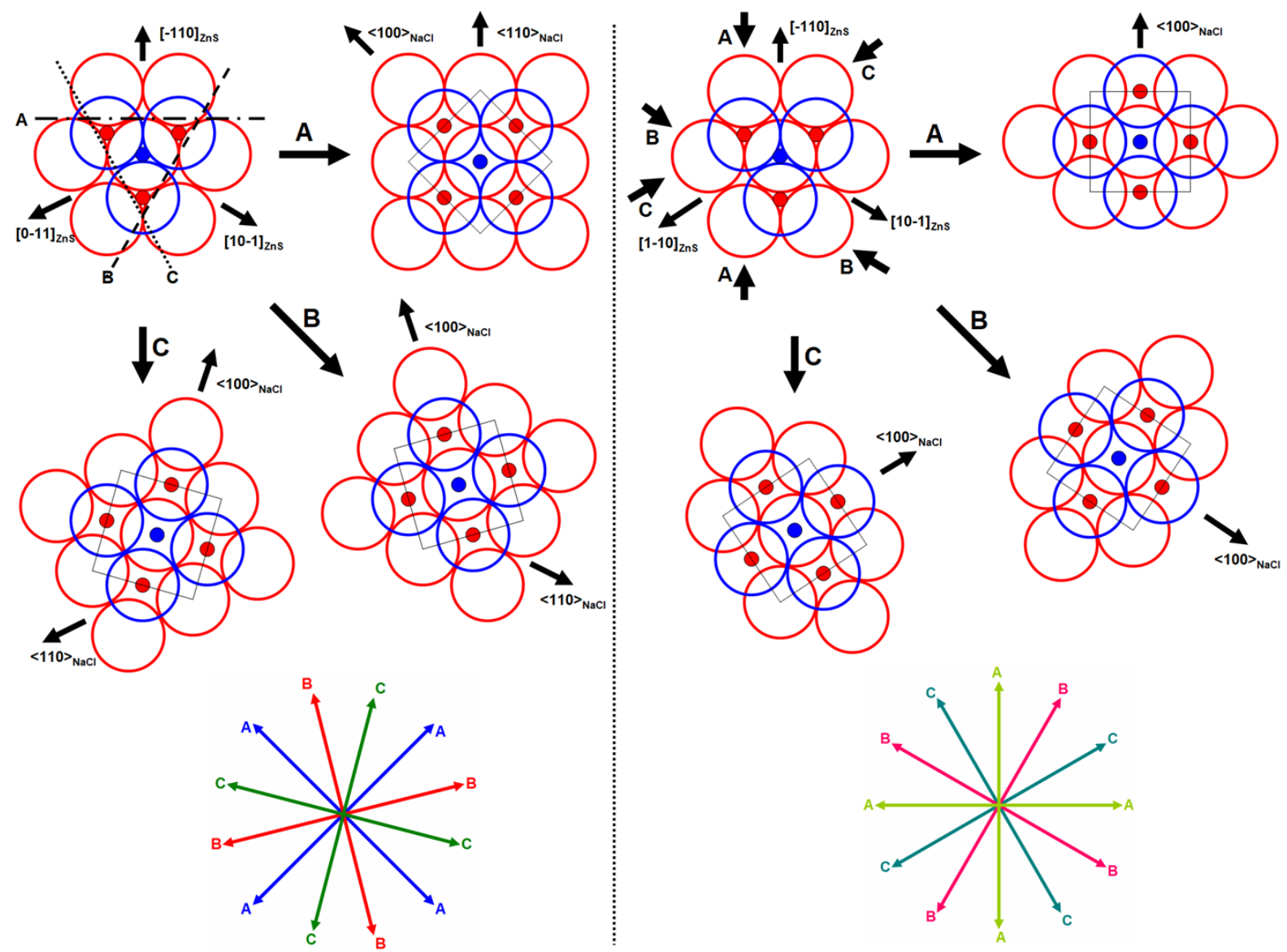

Abbildung 7.9: Graphische Darstellung des Transformationsmechanismus senkrecht zur $[111]_{Z n S^{-}}$ Richtung der Niederdruckphase. Die leeren Kreise stellen die Anionen dar, die gefüllten Kreise die Kationen. Kationen und Anionen, die zu einer Ebene gehören, sind gleichfarbig dargestellt. Die dünnen schwarzen Linien zeigen die jeweilige Lage der Elementarzelle der Hochdruckphase. Links der Mechanismus der anhand der Verschiebungen der Doppelschichten entlang der Geraden A, B, C zu den drei verschiedenen $\langle 110\rangle_{Z n S} \|\langle 110\rangle_{N a C l}$-Orientierungen führt, rechts der Mechanismus, der hauptsächlich durch Kompression entlang der $\langle 110\rangle_{Z n S}$-Richtungen $\mathrm{zu}$ den drei verschiedenen $\langle 110\rangle_{\mathrm{ZnS}} \|\langle 100\rangle_{\mathrm{NaCl}}$-Orientierungen führt. Unten sind die jeweils aus den einzelnen Mechanismen resultierenden Orientierungen der $\langle 100\rangle$-Richtungen der Hochdruckphase dargestellt, die um $30^{\circ}$ um die $[001]_{N a C l}$-Richtung gegeneinander verdreht sind. Die Orientierungen beider Mechanismen kombiniert ergeben einen Winkel von $15^{\circ}$ zwischen den einzelnen Orientierungen. 
$\mathrm{Zu}$ den als energetisch günstig für einen Phasenübergang vom Zinkblende- zum $\mathrm{NaCl}$ Typ [30] geltenden Phasenübergänge gehören die beiden von H. Sowa vorgeschlagenen

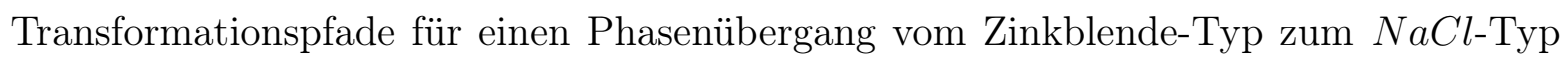
über die gemeinsamen Untergruppen $P 3_{2}$ [70] und $I m m 2$ [68]. Beide entsprechen jedoch nicht den Gegebenheiten der resultierenden Orientierungsbeziehungen. Ein Phasenübergang über die gemeinsame Untergruppe $P 3_{2}$ entspricht einer Rotation der Elementarzelle um $180^{\circ}$ um eine der $\langle 111\rangle_{Z n S}$-Richtungen, was bedeutet, dass eine dieser Richtungen bei der Umwandlung in den $\mathrm{NaCl}$-Typ erhalten bleiben müsste und es keine Übereinstimmungen bei $\langle 111\rangle_{Z n S}$-Richtungen der Niederdruckphase und $\langle 100\rangle_{N a C l}$-Richtungen der Hochdruckphase gibt. Bei einem Phasenübergang über die gemeinsame Untergruppe Imm2 ist die Elementarzelle der Hochdruckphase gegenüber der Niederdruckphase um $90^{\circ}$ verkippt, sodass beispielhaft die [001]-Richtung des Zinkblende-Typs parallel zur [1110]-Richtung des $\mathrm{NaCl-Typs} \mathrm{liegt,} \mathrm{und} \mathrm{die} \mathrm{[110]-Richtung} \mathrm{des} \mathrm{Zinkblende-Typs} \mathrm{paral-}$ lel zur [001]-Richtung des $N a C l$-Typs. Der Winkel zwischen den $\langle 111\rangle$-Richtungen des Zinkblende-Typs und den $\langle 100\rangle$-Richtungen des $\mathrm{NaCl}$-Typs beträgt etwa $10^{\circ}$. Hatch et al. [30] und Capillas et al. [16] schlagen zusätzlich zu den Transformationspfaden über Imm2 und $P 3_{2}$ eine Reihe weiterer Transformationspfade vor, von denen jedoch auch keiner auf die für InAs bestimmten Orientierungsbeziehungen passt. Grundsätzlich lässt sich aus den Berechnungen von Hatch et al. [30] jedoch erkennen, dass Transformationspfade mit niedriger Aktivierungsenergie häufig mit einer sehr niedrigsymmetrischen gemeinsamen Untergruppe einher gehen, weshalb ein solcher, noch unbekannter Transformationspfad auch für den Phasenübergang des InAs eine Möglichkeit ist. 


\subsection{Cadmiumsulfid $(C d S)$ und Cadmiumselenid $(C d S e)$}

An Cadmiumsulfid und Cadmiumselenid wurden bereits einige experimentelle Studien zur Suche von Zwischenphasen während des Phasenübergangs vom Wurtzit-Typ in den NaCl-Typ durchgeführt. Mehrere von ihnen (Knudson et al. [37], Sharma et al. [64], Tolbert et al. [82], Wickham et al. [89]) wurden bereits in Kapitel 2.7.2 kurz beschrieben. Explizit der Suche nach Orientierungsbeziehungen zwischen Hoch- und Niederdruckphase dienten die beiden Arbeiten von Sowa ([72], [73]) an $C d S$ und $C d S e$. Mit Hilfe einer Präzessionskamera und Röntgenfilm wurde die Orientierung der Niederdruckphase und auf der Basis der $200_{H P}$-Reflexe die Orientierung der Hochdruckphase bestimmt. Sowohl für $C d S$ als auch für $C d S e$ wurde aufgrund der Ergebnisse ein Transformationspfad über die gemeinsame Untergruppe $C m c 2_{1}$ vermutet.

Am Material Cadmiumsulfid wurden drei Experimente durchgeführt:

- Die Messungen der Probe juli09sept09 wurden an der Beamline BW5 in der kleinen Vierschraubenzelle durchgeführt. Als Detektor kam der mar345 Flächendetektor zum Einsatz. Der abgedeckte Winkelbereich betrug $-45^{\circ} \leq \omega \leq 45^{\circ}$, mit $\Delta \omega=1^{\circ}$. Der Einkristall wurde bei einem Druck von 2.1 GPa gemessen, für die Hochdruckphase ist der Druck unbekannt, da im HASYLAB zum Zeitpunkt des Experiments keine Ausrüstung zur Druckmessung vorhanden war. Die Wellenlänge betrug $\lambda=0.1246 \AA$, der Probe-Detektor-Abstand $1441.5 \mathrm{~mm}$. Bei der auf 1.0 GPa entlasteten Niederdruckphase betrug die Wellenlänge $\lambda=0.1255 \AA$ und der Probe-Detektor-Abstand $1440 \mathrm{~mm}$.

- Das Experiment mit der Probe sept10 wurde in der Las1-Zelle an der Beamline BW5 durchgeführt. Als Detektor wurde der mar345 Flächendetektor genutzt. Der Einkristall wurde bei einem Druck von 1.8 GPa in einem Winkelbereich von $-45^{\circ} \leq \omega \leq 45^{\circ}$ gemessen. Der Druck für die Hochdruckphase und die entlastete Niederdruckphase ist unbekannt, da im HASYLAB zum Zeitpunkt des Experiments keine Ausrüstung zur Druckmessung vorhanden war. Diese beiden Phasen wurden in einem Winkelbereich von $-40^{\circ} \leq \omega \leq 40^{\circ}$ gemessen. Alle Messungen wurden mit der Wellenlänge $\lambda=0.1248 \AA$ und einem Probe-Detektor-Abstand von 
1370 mm durchgeführt.

- Die Messungen der Probe juni13 wurden an der Beamline P02.1 in der Las1-Zelle durchgeführt. Als Detektor kam das Perkin Elmer 1621-Modell zum Einsatz. Der Einkristall wurde bei einem Druck von 1.1 GPa, die Hochdruckphase bei einem Druck von 4.2 GPa und die entlastete Niederdruckphase bei einem Druck von 0.9 GPa gemessen. Alle Messungen wurden mit einem gemessenen Winkelbereich von $-42^{\circ} \leq \omega \leq 42^{\circ}$, einer Wellenlänge von $\lambda=0.2073 \AA$ und einem Probe-DetektorAbstand von $1008.35 \mathrm{~mm}$ durchgeführt.
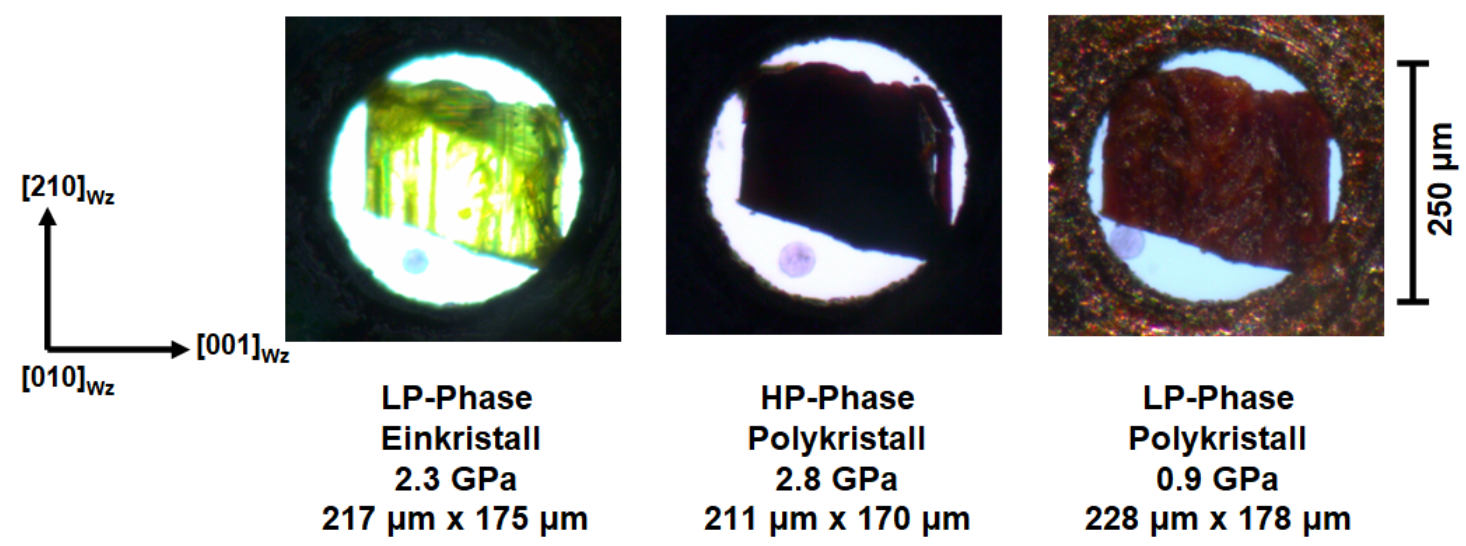

Abbildung 7.10: Aufnahmen der $C d S$-Probe des Experiments juni13. Das Koordinatensystem auf der linken Seite zeigt die grobe Orientierung des Einkristalls zu Versuchsbeginn an. Deutlich zu sehen ist der Farbwechsel der Probe vom transparenten Gelb des Einkristalls zum wenig durchscheinenden Rot der entlasteten polykristallinen Niederdruckphase.

Während der Phasenumwandlung vom Einkristall zur Hochdruckphase wurde bei der $C d S$-Probe des Experiments juni13 eine Schrumpfung um $6 \mu \mathrm{m}$ in $[001]_{W z^{-R i c h t u n g}}$ und um $5 \mu \mathrm{m}$ in $[210]_{W z}$-Richtung beobachtet. Dies entspricht $4 \%$ in $[001]_{W z}$ bzw. $7 \%$ in $[210]_{W z}$. Bei der Rückumwandlung in die Niederdruckphase wurde eine entsprechende Ausdehnung festgestellt. Abbildung 7.10 zeigt die dazugehörigen Mikroskopaufnahmen.

Am Cadmiumselenid wurden zwei Experimente durchgeführt:

- Das Experiment mai11 wurde in der Las1-Zelle an der Beamline BW5 durchgeführt. Der Druck bei der Messung des Einkristalls betrug 1.8 GPa, für die Hochdruckphase ist der Druck unbekannt, da im HASYLAB zum Zeitpunkt des Experiments keine Ausrüstung zur Druckmessung vorhanden war. Um den 200-Reflex 
des Stahlgaskets auszublenden, wurden zusätzliche Messungen der Hochdruckphase mit einer Lochblende durchgeführt. Es wurde ein Bereich von $-40^{\circ} \leq \omega \leq 40^{\circ}$ abgedeckt. Die Schrittweite $\Delta \omega$ bei der Einkristallmessung und den Hochdruckphasenmessungen ohne Blende betrug $0.5^{\circ}$, bei den Messungen mit Blende betrug sie $1^{\circ}$. Der Probe-Detektor-Abstand betrug $1290.9 \mathrm{~mm}$, die Wellenlänge $\lambda=0.12395$

$\AA$. Es wurde keine entlastete Niederdruckphase gemessen.

- Das Experiment jul12 wurde in der Kleinen Vierschraubenzelle als erster Versuch eines Hochdruckexperiments an der Beamline P02.1 durchgeführt. Zuerst wurde der Einkristall bei einem Druck von 1.5 GPa gemessen. Anschließend wurde der Druck auf 4.0 GPa erhöht und es kam zur Phasenumwandlung. Nach der Messung in diesem Zustand wurde der Druck auf 0.9 GPa abgesenkt und es fand eine teilweise Rückumwandlung in die Niederdruckphase statt. Sämtliche Messungen wurden mit einer Schrittweite $\Delta \omega=0.5^{\circ}$ durchgeführt, der abgedeckte Bereich war $-42^{\circ} \leq \omega \leq 42^{\circ}$. Der Probe-Detektor-Abstand betrug $730.023 \mathrm{~mm}$, die Wellenlänge $\lambda=0.20723 \AA$. Die Polfiguren des Experiments waren größtenteils nicht auswertbar, da aufgrund der noch unbekannten Beamline die Justierung der Druckzelle im Strahl noch nicht ausgereift war und auch noch keine passende Halterung für die kleine Vierschraubenzelle zur Verfügung stand.

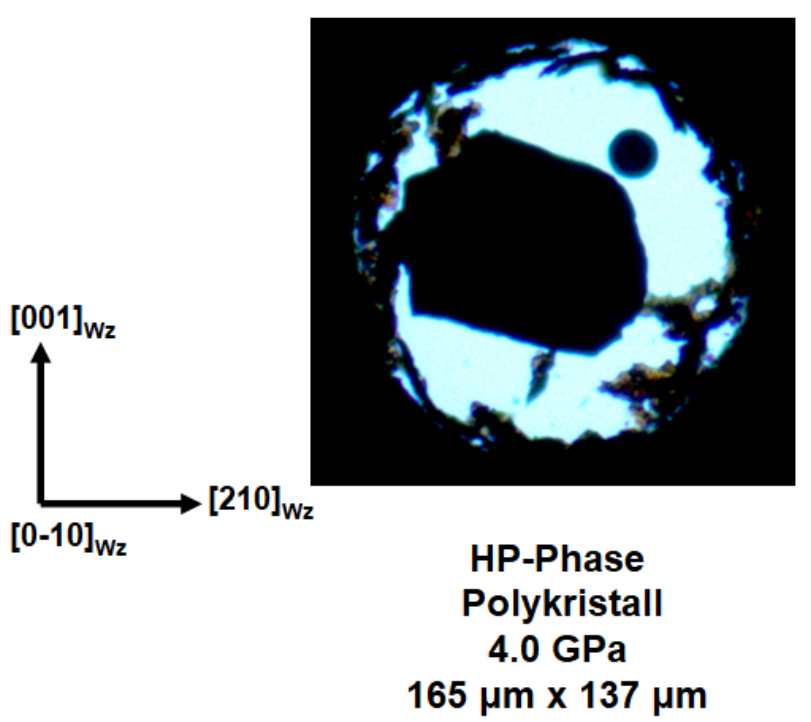

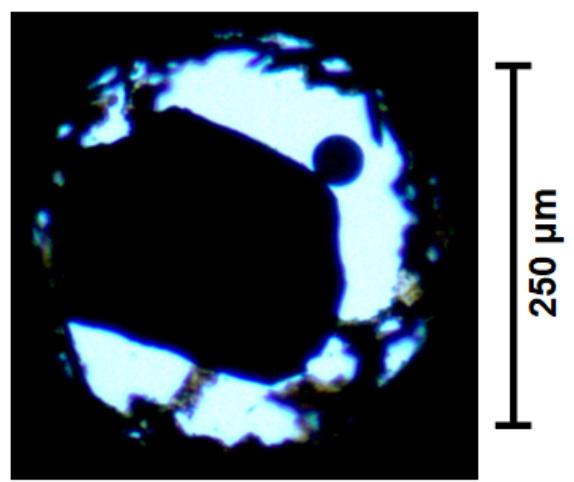

LP-Phase

Polykristall

$0.9 \mathrm{GPa}$

$190 \mu \mathrm{m} \times 163 \mu \mathrm{m}$

Abbildung 7.11: Aufnahmen der $C d S e$-Probe des Experiments jul12. Das Koordinatensystem auf der linken Seite zeigt die grobe Orientierung des Einkristalls zu Versuchsbeginn an.

Zwischen Niederdruck- zur Hochdruckphase wurde bei der $C d S e$-Probe des Experiments 


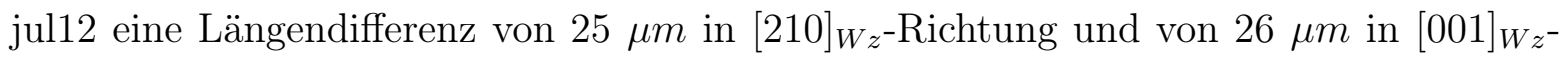
Richtung beobachtet, wobei die Hochdruckphase die niedrigeren Probenlängen aufwies. Dies entspricht $13 \%$ in $[210]_{W z}$ bzw. $16 \%$ in $[001]_{W z}$. Abbildung 7.11 zeigt die dazugehörigen Mikroskopaufnahmen.
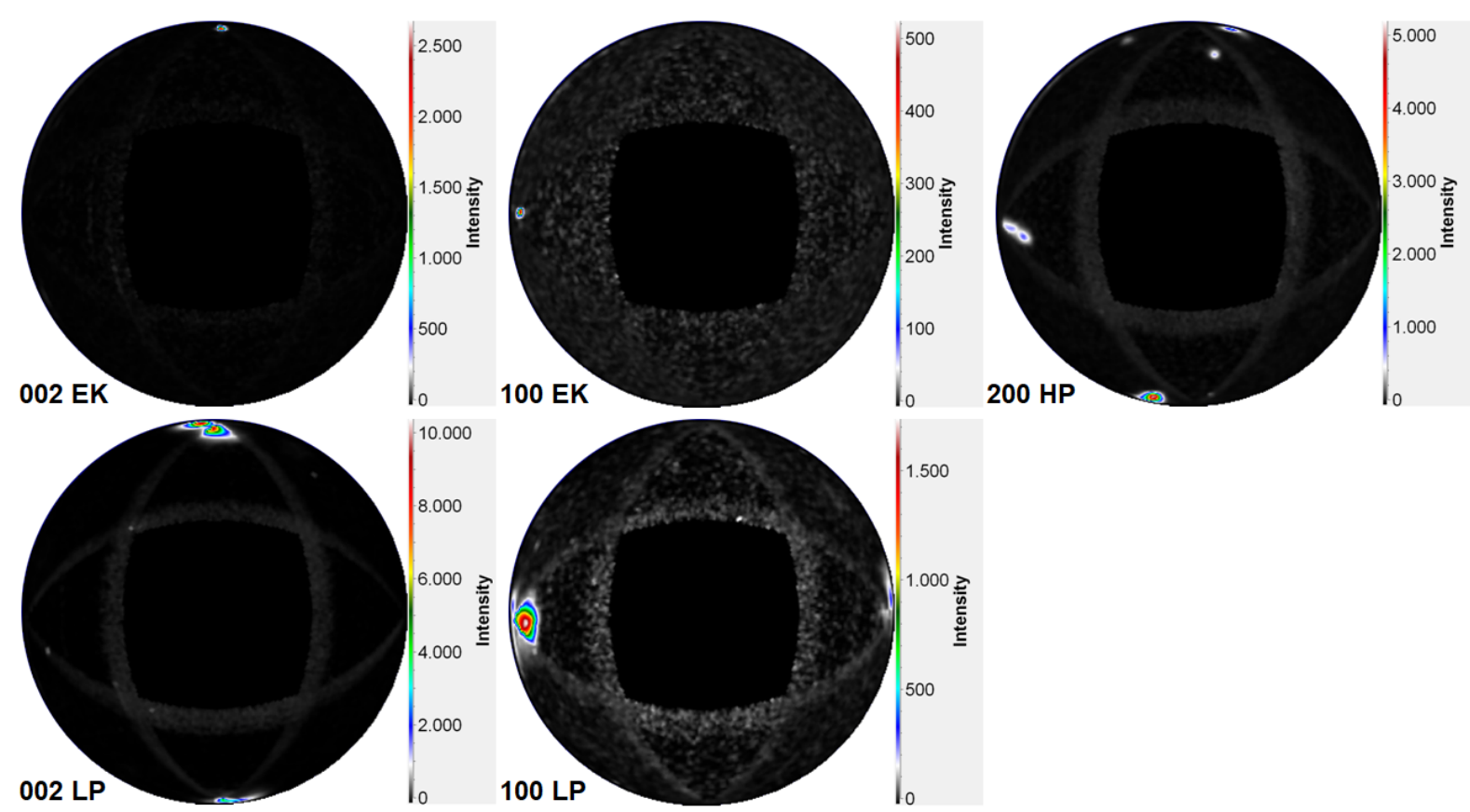

Abbildung 7.12: Ausgewählte Polfiguren des $C d S$-Experiments juli09.

Abbildung 7.12 zeigt die Polfiguren der $C d S$-Probe juli09. Der Einkristall dieser Probe ist mit der Längsrichtung in $[001]_{W z}$-Richtung orientiert, in Querrichtung zeigt der Flächenpol der $\{100\}_{W z}$-Ebene, was im hexagonalen Kristallsystem der $[210]_{W z}$-Richtung entspricht. Die gemessene $\{100\}_{\mathrm{NaCl}}$-Polfigur der Hochdruckphase zeigt einen deutli-

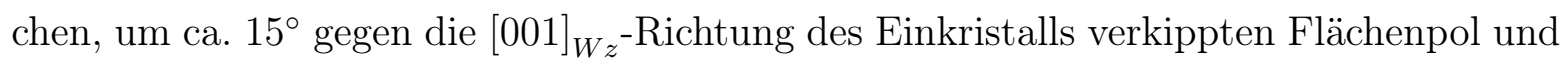
einen sehr schwachen, verschmierten Flächenpol mit zwei Maxima nahe der Äquatorebene. Die entlastete Niederdruckphase weist die Orientierung des Einkristalls als einzige Orientierung auf.

Abbildung 7.13 zeigt die Polfiguren der $C d S$-Probe sept10. Der Einkristall dieser Probe ist mit der Längsrichtung in $[001]_{W z}$-Richtung orientiert, in Querrichtung liegt der Flächenpol einer $\{100\}_{W z}$-Ebene, was im hexagonalen Kristallsystem einer $\langle 210\rangle_{W z^{-}}$ Richtung entspricht. In der Hochdruckphase zeigen sich insgesamt sechs verschiedene Orientierungen der $\{100\}_{\mathrm{NaCl}^{-}}$-Flächenpole mit unterschiedlicher Intensität in einem Winkelabstand von ca. $15^{\circ}$ um die $[001]_{W z^{-R i c h t u n g}}$ des Einkristalls herum. Der Win- 


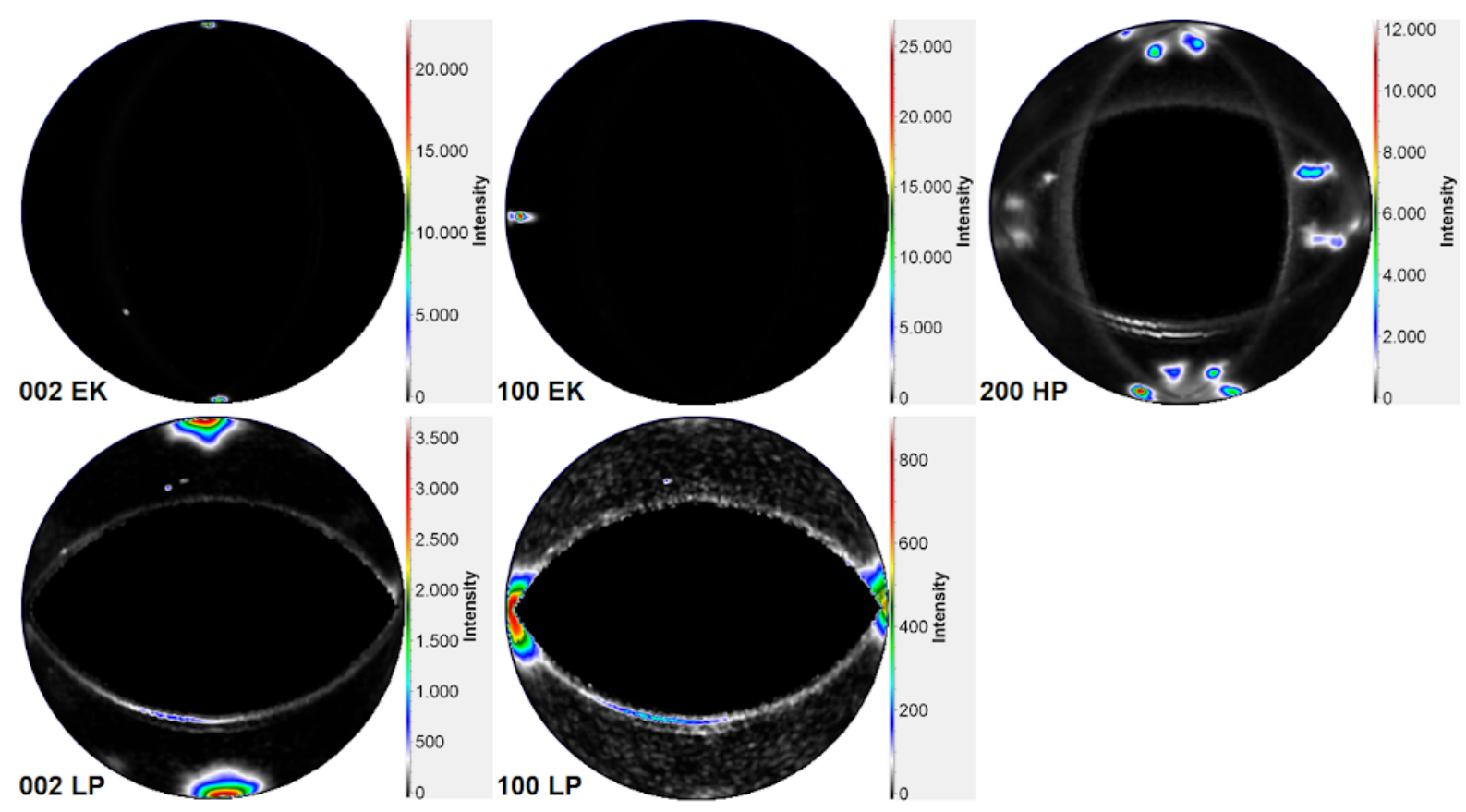

Abbildung 7.13: Ausgewählte Polfiguren des $C d S$-Experiments sept10.

kel zwischen den einzelnen $\{100\}_{\mathrm{NaCl}}$-Flächenpolen beträgt ca. $60^{\circ}$. Die Richtungen der $15^{\circ}$-Kippungen entsprechen den $\langle 210\rangle_{W z}$-Richtungen des Einkristalls. In der Nähe der Äquatorebene sind zwei Paare schwächerer $\{100\}_{\mathrm{NaCl}}$-Flächenpole zu erkennen, die jeweils im $90^{\circ}$-Winkel zu einem der um $[001]_{W z}$ orientierten $\{100\}_{\mathrm{NaCl}^{-}}$-Flächenpole stehen und in Querrichtung über einen Bereich von ca. $15^{\circ}$ verschmiert sind. Die entlastete Niederdruckphase weist nur die ursprüngliche Orientierung des Ausgangseinkristalls auf.

Abbildung 7.14 zeigt die Polfiguren der $C d S$-Probe juni13. Beim Einkristall zeigt im Gegensatz zu den übrigen Proben die $[001]_{W z}$-Richtung in die Querrichtung, in Längsrichtung liegt der Flächenpol einer $\{100\}_{W z}$-Ebene, was einer $\langle 210\rangle_{W z}$-Richtung entspricht. In den Polfiguren der Hochdruckphase sind zwei Orientierungen der $\{100\}_{\mathrm{NaCl}^{-}}$ Flächenpole zu erkennen, die jeweils um etwa $13^{\circ}$ auf dem $90^{\circ}$-Großkreis gegen die $[001]_{W z}$-Richtung verkippt sind. Weitere Orientierungen sind im Gegensatz zum Experiment sept10 nicht zu sehen. Die entlastete Niederdruckphase ist wieder genauso wie der ursprüngliche Einkristall orientiert.

Abbildung 7.15 zeigt relevante ODF-Schnitte der $C d S$-Probe juni13. Alle ODFs wurden mit orthorhombischer Probensymmetrie berechnet, die sich in den Polfiguren gut 


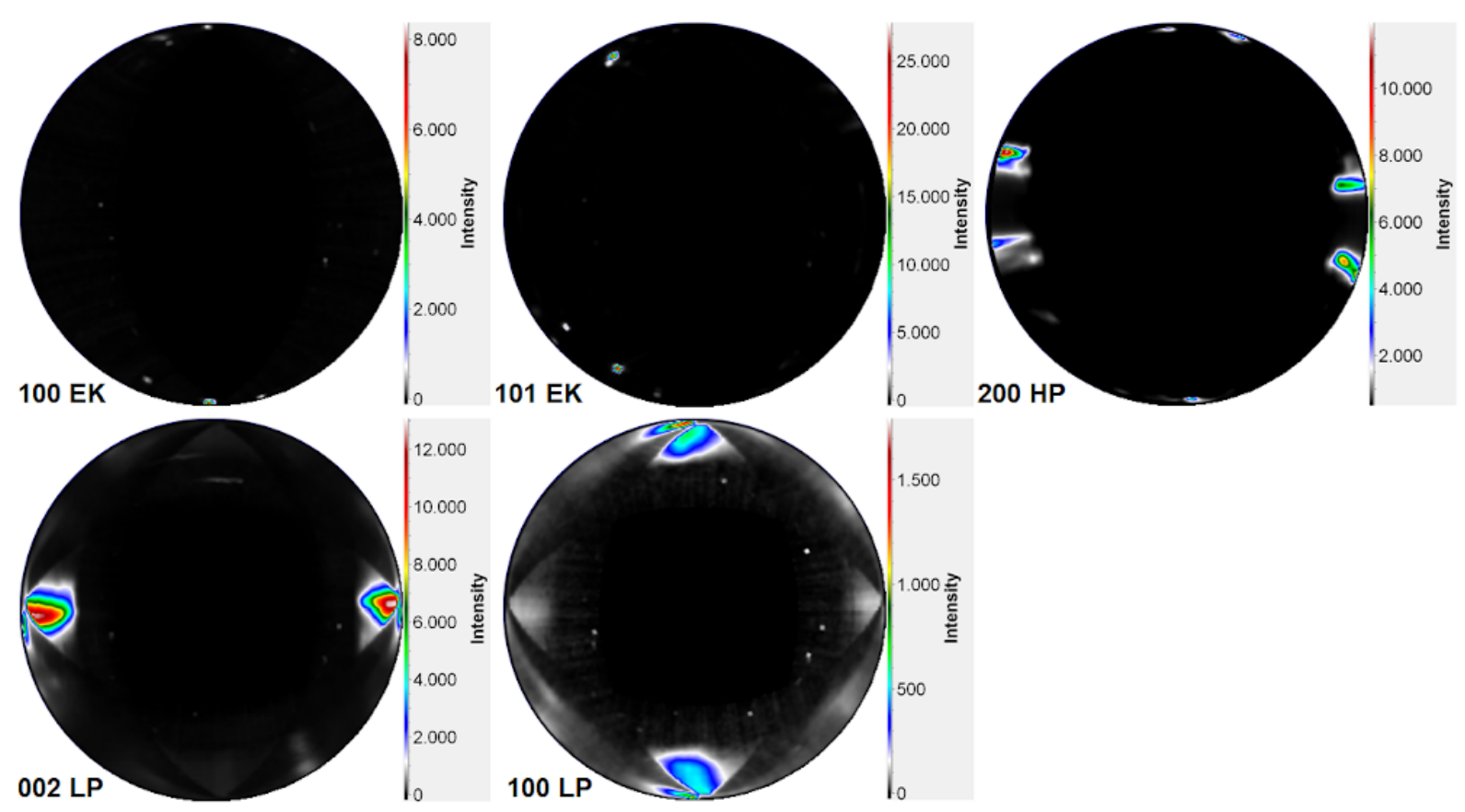

Abbildung 7.14: Ausgewählte Polfiguren des $C d S$-Experiments juni13.
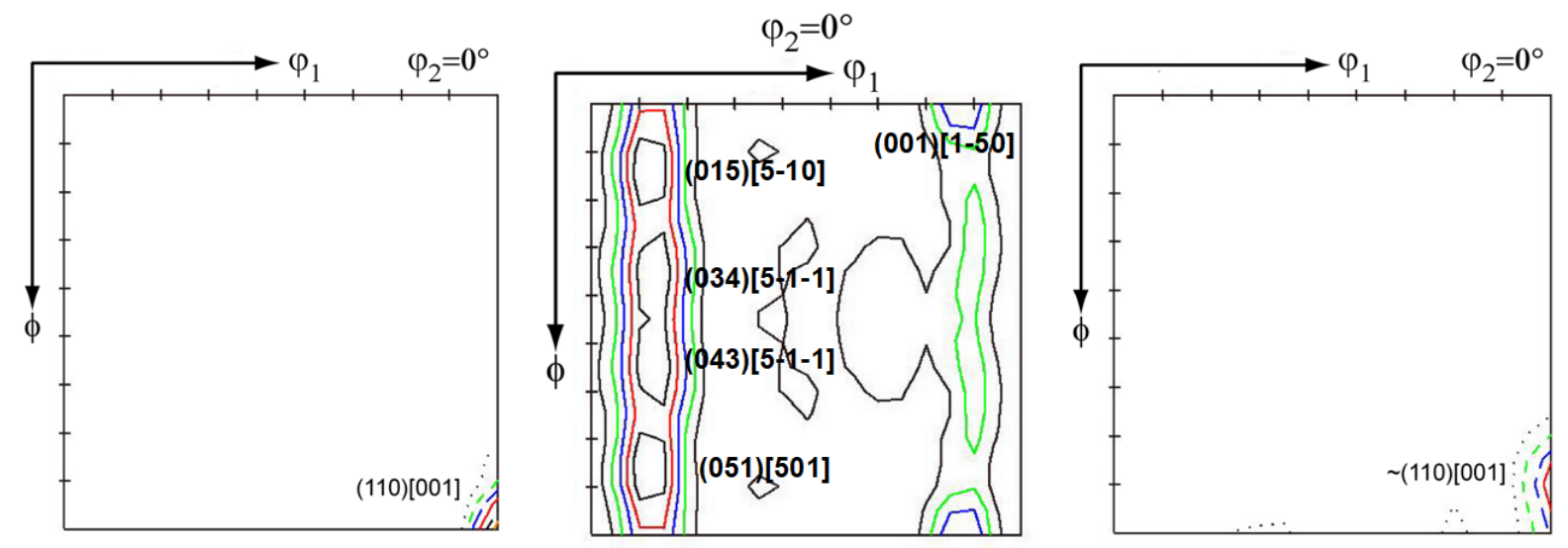

Abbildung 7.15: ODF-Schnitte des $C d S$-Experiments juni13 für $\varphi_{2}=0^{\circ}$. Links der hexagonale Einkristall, in der Mitte die kubische Hochdruckphase, rechts die entlastete hexagonale Niederdruckphase. 
erkennen lässt. Die Kristallsymmetrie des Ausgangseinkristalls und der entlasteten Niederdruckphase ist hexagonal, die Kristallsymmetrie der Hochdruckphase ist kubisch. Der Reihenentwicklungsgrad betrug für alle Phasen $L_{\max }=22$. Die Einkristall-Ausgangstextur der Probe im hexagonalen Wurtzit-Typ weist ausschließlich eine starke \{90, 90, 0\}-Texturkomponente auf. In der $(h k l)[u v w]$-Beschreibung entspricht dies einer (110) [001]-Textur.

Die Hochdruckphase im $\mathrm{NaCl}$-Typ zeigt dagegen eine sehr komplexe Orientierungsverteilung. Zur Beschreibung genügt es, den $\varphi_{2}$-Schnitt von $0^{\circ} \mathrm{zu}$ betrachten. Eine Komponente ist eine nicht ideale $\Phi$-Faser $\left\{\varphi_{1}=12^{\circ}, 0^{\circ} \leq \Phi \leq 90^{\circ}, \varphi_{2}=0^{\circ}\right\}$, die etwa mit $(h k l)$ [510] indiziert werden kann. Die Faser zeigt Intensitätsmaxima vom Typ (015) [510] und (043) [511]. Der Winkel von $\varphi_{1}=12^{\circ}$ stimmt mit der gemessenen Auslenkung der $\{100\}_{N a C l}$-Flächenpole gegen die $[001]_{W z}$-Richtung überein, die errechneten nicht idealen Fasern sind jedoch vor dem Hintergrund der gemessenen Polfiguren (siehe Abbildung 7.14 sehr wahrscheinlich nicht aussagekräftig.

Die entlastete Niederdruckphase zeigt wieder nur eine fast ideale Komponente, die nahezu die Orientierung des Einkristalls vor der Phasenumwandlung annimmt. Diese Orientierungslage befindet sich in der Nähe der idealen Lage (110) [001], die exakte Lage befindet sich bei den Eulerwinkeln $\{90,83,0\}$.

Durch die Berechnung der ODF ließ sich die einfache Orientierungsverteilung des Einkristalls und der entlasteten Niederdruckphase gut bestimmen, für die Hochdruckphase konnte aufgrund der geringen Polfigurabdeckung und der mangelnden Qualität der gemessenen Polfiguren jedoch nur eine grobe Bestimmung der Hauptorientierung erreicht werden.

Abbildung 7.16 zeigt die Polfiguren der $C d S e$-Probe mai11. Der Einkristall dieser Probe ist mit der Längsrichtung in $[001]_{W z}$-Richtung orientiert, in Querrichtung zeigt der Flächenpol der $\{100\}_{W z}$-Ebene, was im hexagonalen Kristallsystem der $[210]_{W z}$-Richtung entspricht. Wie im $C d S$-Experiment sept10 ist für die Hochdruckphase die sechsfache Aufspaltung eines der $\{100\}_{\mathrm{NaCl}}$-Flächenpole in $60^{\circ}$-Schritten um die $[001]_{W z^{-R i c h t u n g}}$ des Einkristalls zu sehen. Auch hier beträgt der Auslenkungswinkel ca. 15. Ebenso sind in Äquatornähe weitere $\{100\}_{\mathrm{NaCl}^{-}}$-Flächenpole zu erkennen, die in Querrichtung um einen Winkelbereich von ca. $15^{\circ}$ verschmiert sind. Diese Flächenpole liegen in einem $90^{\circ}$-Winkel zu einem der $[001]_{W z}$-nahen Flächenpole. Es wurde keine entlastete Niederdruckphase gemessen, Sowa [73] fand nach der Rückumwandlung breite Reflexe einer Phase, die die Orientierung des Einkristalls aufwies und dem Wurtzit-Typ zugeordnet wurde. Das gleichzeitige Auftreten des $Z n S$-Typs als zweite Niederdruckphase konnte 


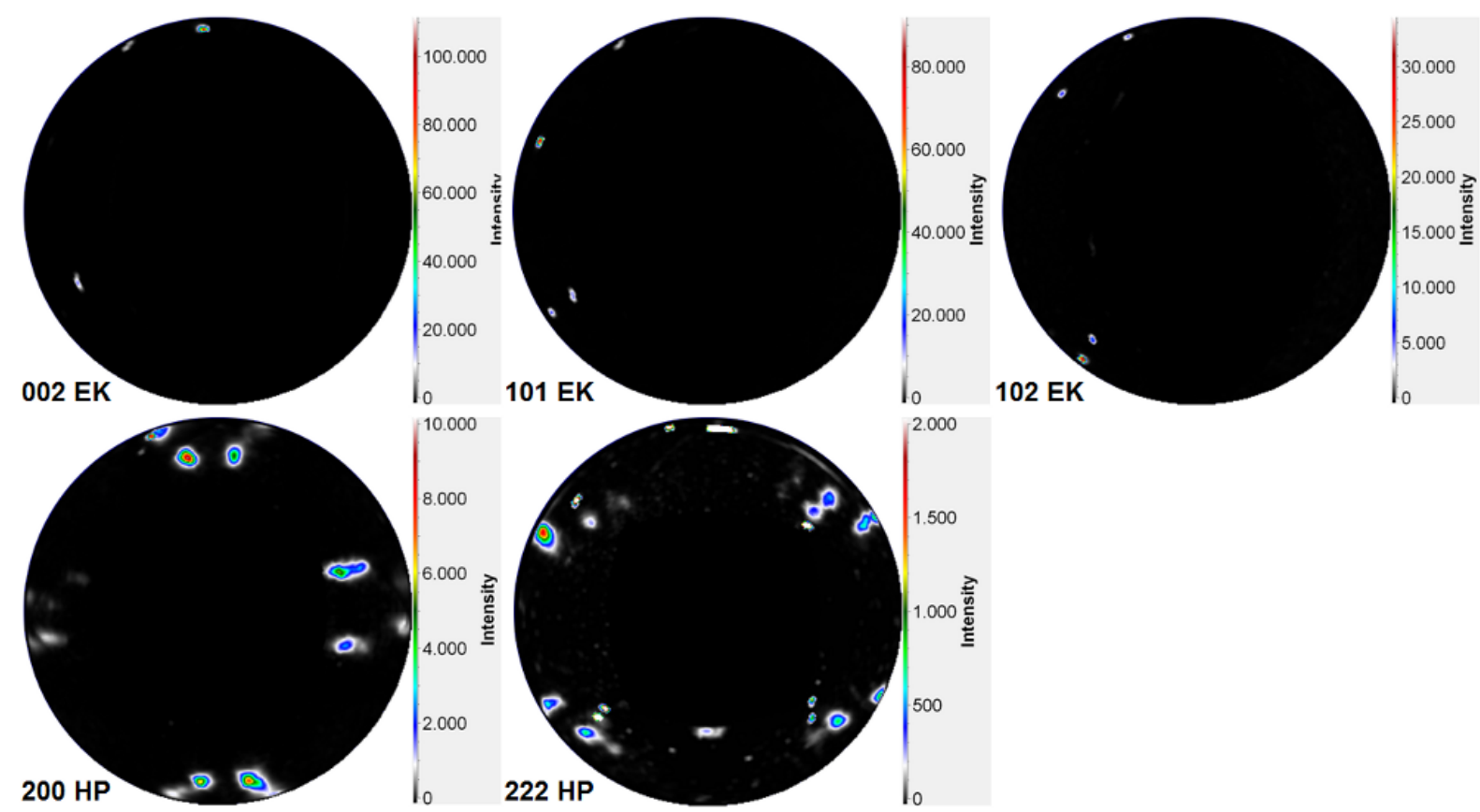

Abbildung 7.16: Ausgewählte Polfiguren des $C d S e$-Experiments mai11.

nicht eindeutig ausgeschlossen werden.

Aus den obigen Beobachtungen wurde für die sechs gefundenen Orientierungen der Hochdruckphase die in Abbildung 7.17 gezeigten idealisierten Orientierungsbeziehungen für eine Orientierung der hexagonalen Ausgangsphase abgeleitet, wie sie im $C d S$ Experiment sept10 und im $C d S e$-Experiment juli12 auftritt (Längsrichtung $[001]_{W z}$, Querrichtung $\left.[210]_{W z}\right)$. Die um die $[001]_{W z}$-Richtung angeordneten $\{100\}_{N a C l}$-Flächenpole sind um ca. $15^{\circ}$ aus dieser Richtung ausgelenkt und haben untereinander einen Winkelabstand von $60^{\circ}$. Sie liegen auf den gleichen Großkreisen wie die $\{100\}_{W z}$-Flächenpole und sind in Abbildung 7.17 von 1 bis 6 durchnummeriert. Für jede dieser sechs Orientierungen wurde ein Großkreis im $90^{\circ}$-Winkel zum jeweiligen $\{100\}_{\mathrm{NaCl}}$-Flächenpol konstruiert, auf dem die zwei noch fehlenden Gitterrichtungen der kubischen Elementarzelle der Hochdruckphase liegen müssen. So ergab sich ein Gürtel von $\pm 15^{\circ}$ um die Äquatorebene für die noch fehlenden Flächenpole. Aus der Auswertung der gemessenen Polfiguren ergeben sich zwei Lagemöglichkeiten für die äquatornahen $\{100\}_{N a C l}$-Flächenpole:

1. um $7^{\circ}$ vom Äquator in $[001]_{W z}$-Richtung ausgelenkt auf dem gleichen Großkreis wie die $\{100\}_{W z}$-Flächenpole und damit auch auf dem gleichen Großkreis wie die $[001]_{W z}$-nahen Flächenpole der Hochdruckphase 


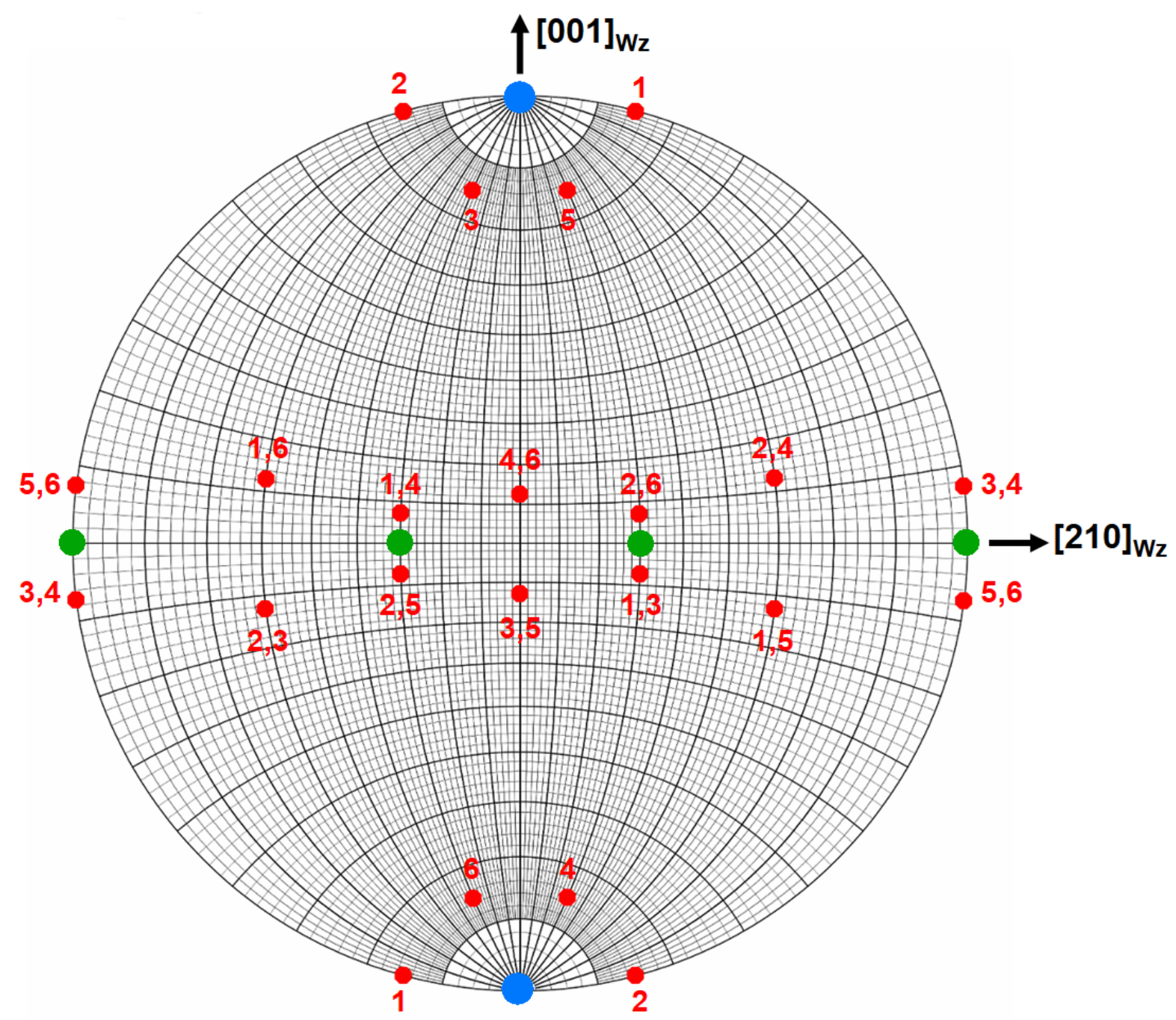

Abbildung 7.17: Idealisierte Darstellung möglicher Orientierungen der Hochdruckphase von $C d S$ und $\mathrm{CdSe}$. Die roten Punkte zeigen die möglichen Lagen der $\{100\}_{\mathrm{NaCl}}$-Flächenpole der Hochdruckphase an und sind nach den sechs möglichen Orientierungen von 1 bis 6 durchnummeriert. Die blauen Punkte zeigen die Lage der $\{001\}_{W z}$-Flächenpole der Niederdruckphase an, die grünen Punkte die Lage der $\{100\}_{W z}$-Flächenpole der Niederdruckphase. 
2. um $12^{\circ}$ vom Äquator in $[001]_{W z^{-R i c h t u n g}}$ ausgelenkt und um $30^{\circ}$ in $[001]_{W z^{-}}$ Richtung

Hierbei gehören jeweils ein Flächenpol in Lage 1 und Lage 2 zu einer Orientierung. In Abbildung 7.17 sind die äquatorialen Flächenpole ihrer jeweils möglichen Orientierung 1 bis 6 zugeordnet. Hierbei fällt auf, dass Flächenpolpositionen auch von mehr als einer Orientierung besetzt werden können und kein äquatorialer Flächenpol einer Orientierung 1 bis 6 mit seinem $[001]_{W z}$-nahen Flächenpol auf einem Großkreis durch $[001]_{W z}$ und $\{100\}_{W z}$ liegt. Eine Lage in der ein $[001]_{W z}$-naher Flächenpol und ein Flächenpol in Äquatornähe der gleichen Orientierung 1 bis 6 auf einem Großkreis durch $[001]_{W z}$ und $\{100\}_{W z}$ liegen (was eine Auslenkung um $15^{\circ}$ für den äquatornahen Flächenpol bedeuten würde), wurde nicht beobachtet.

Im Experiment wurde sowohl bei $C d S$ als auch bei $C d S e$ eine 'Verschmierung' der äquatornahen $\{100\}_{\mathrm{NaCl}}$-Flächenpole von ca. $\pm 7^{\circ}$ in $[210]_{W z}$-Richtung um die in Abbildung 7.17 gezeigten idealen Lagen beobachtet, die manchmal sogar schwache zwei Maxima aufwies, eines auf jeder Seite der idealen Lage.

Die Auslenkung der $\{100\}_{N a C l}$-Flächenpole aus der $[001]_{W z}$-Richtung um ca. $15^{\circ}$ stimmt mit den Beobachtungen von Sowa [72],[73] sowohl für $C d S$ als auch für $C d S e$ überein. Ebenso bestätigte sich die von Sowa für $C d S e$ gemachte Beobachtung der verschmierten Flächenpole in Äquatornähe, die gelegentlich sogar in zwei Maxima aufgelöst werden konnten, in den vorliegenden Experimenten sowohl für $C d S$ als auch für $C d S e$.

Es wird angenommen, dass die $15^{\circ}$-Verkippung durch Spannungen während der Transformation zwischen umgewandelten und noch nicht umgewandelten Regionen verursacht wird [72], welche ihre Ursache in der Volumenreduktion von ca. 20\% für die Umwandlung vom Wurtzit-Typ in den $\mathrm{NaCl-Typ} \mathrm{haben} \mathrm{[42].} \mathrm{Diese} \mathrm{Kräfte} \mathrm{könnten} \mathrm{auch} \mathrm{eine} \mathrm{Ursache}$ für die Verschmierung der äquatornahen $\{100\}_{\mathrm{NaCl}}$-Flächenpole sein.

Werden die Verkippung der $\{100\}_{\mathrm{NaCl}}$-Flächenpole um $15^{\circ}$ gegen die $[001]_{W z}$-Richtung und die Verschmierung der äquatornahen $\{100\}_{N a C l}$-Flächenpole nicht berücksichtigt, ergeben sich folgende ideale Orientierungsbeziehungen:

$$
\begin{aligned}
& \langle 001\rangle_{W z} \|\langle 100\rangle_{N a C l} \\
& \langle 100\rangle_{W z} \|\langle 100\rangle_{N a C l} \\
& \langle 120\rangle_{W z} \|\langle 100\rangle_{N a C l}
\end{aligned}
$$




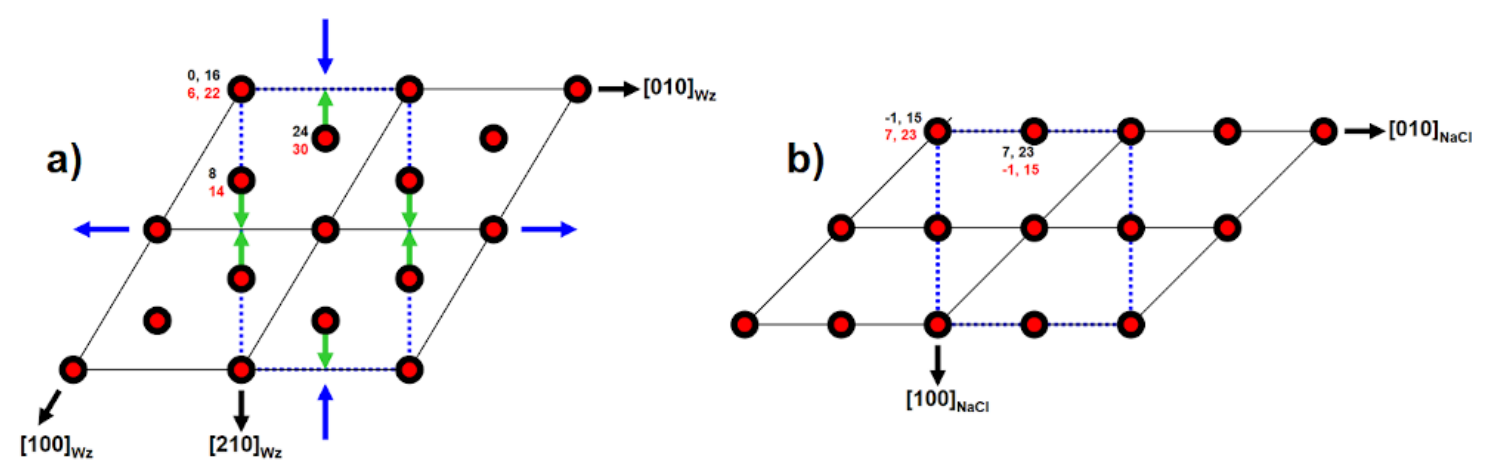

Abbildung 7.18: Schematische Darstellung der atomaren Verschiebungen des Phasenübergangs vom a) Wurtzit-Typ in den b) $N a C l$-Typ über die gemeinsame Untergruppe $C m c 2_{1}$ mit Blickrichtung parallel zur [001]-Richtung beider Phasen. Die Kationen sind rot, die Anionen schwarz dargestellt. Die $z$-Koordinaten der Atome in der Elementarzelle sind in $n / 32$ angegeben. Die grünen Pfeile kennzeichnen die Atombewegungen, die blauen Pfeile kennzeichnen die Metrikänderungen des Gitters. Die blaue gestrichelte Linie definiert die (künftige) Elementarzelle des $N a C l$-Typs. (Quelle: Sowa [73], modifiziert).

Diese Orientierungsbeziehungen werden für den Transformationspfad vom Wurtzit-Typ in den $\mathrm{NaCl}$-Typ über die gemeinsame Untergruppe $C m c 2_{1}$ erhalten. Dieser Transformationspfad wurde von Sowa [69] aus Kugelpackungsüberlegungen hergeleitet. Capillas et al. [16] und Stokes et al. [80] bewerten diesen Transformationspfad ebenfalls als energetisch günstig, da keine Bindungen aufgebrochen werden müssen. Der $C m c 2_{1}$ Transformationspfad entspricht einer Kompression der Elementarzelle des Wurtzit-Typs in $[210]_{W z}$-Richtung, während gleichzeitig in $[010]_{W z}$-Richtung eine Elongation stattfindet [69] (siehe Abbildung 7.18). Aufgrund der Symmetrie der hexagonalen Elementarzelle ist dieser Mechanismus in allen symmetrisch äquivalenten Richtungen möglich, sodass am Ende drei mögliche Einzelorientierungen existieren und deshalb erwartet wird, dass sich die Effekte der Längenänderungen größtenteils kompensieren.

Aufgrund der Orientierung der Probe in der Druckzelle konnte die Längenänderung der $[010]_{W z}$-Richtung nicht bestimmt werden, für die $[210]_{W z}$-Richtung wurde eine Kontraktion von $7 \%(C d S)$ bzw. $13 \%(C d S e)$ gemessen. In $[001]_{W z}$-Richtung betrug die Kontraktion $4 \%$ für $C d S$ bzw. 16\% für CdSe. Die aus den Gitterparametern bestimmte erwartete Kontraktion in $[001]_{W z}$-Richtung beträgt $17.8 \%$ für $C d S$ und $20.1 \%$ für $C d S e$. Insgesamt fielen die Metrikänderungen für $C d S$ wesentlich geringer aus als für $C d S e$, wobei die Längenänderung in $[001]_{W z}$-Richtung für $C d S e$ in der Nähe des erwarteten Bereichs liegt.

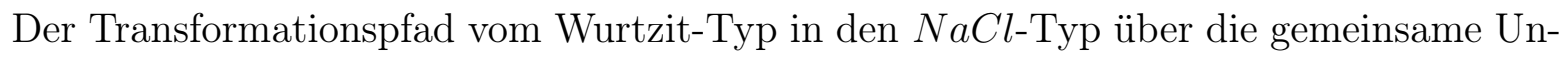
tergruppe Pna2 $2_{1}$ [71] weist ebenfalls die Orientierungsbeziehung $\langle 001\rangle_{W z} \|\langle 100\rangle_{N a C l}$ auf, die dazugehörigen weitere Orientierungsbeziehung $\langle 210\rangle_{W z} \|\langle 110\rangle_{N a C l}$ wurde je- 
doch nicht beobachtet.

Die Ergebnisse der Experimente mit $C d S$ und $C d S e$ bestätigen somit die Beobachtungen, die von Sowa ([72],[73]) auf der Basis von Präzessions- und Debye-ScherrerAufnahmen auf Röntgenfilm gemacht wurden. 


\subsection{Kupferindiumdiselenid ( $\left.\mathrm{CuInSe} \mathrm{I}_{2}\right)$}

Bisher existieren keine Studien zu Orientierungsbeziehungen zwischen Niederdruck- und Hochdruckphase von $\mathrm{CuInSe}$. Bisherige Studien dienten der Strukturbestimmung der Hochdruckphase (Tinoco at al. [81], Bovornratanaraks et al. [8]) oder der Änderung der physikalischen Eigenschaften durch den Phasenübergang wie zum Beispiel das elektrische Transportverhalten (Ye et al. [92]) oder die Schwingungsmoden der Atombindungen (Ves et al. [84], González et al. [25]).

Am Kupferindiumdiselenid wurde ein erfolgreiches Experiment im Juni/Juli 2012 an der Beamline BW5 in der Las1-Diamantstempelzelle durchgeführt. Hierbei wurde ein Bereich des Winkels $\omega$ von $-42^{\circ}$ bis $42^{\circ}$ abgedeckt. Die Schrittweite betrug beim Einkristall $\Delta \omega=0.5^{\circ}$ und bei beiden Polykristallmodifikationen $\Delta \omega=0.2^{\circ}$. Die Wellenlänge betrug $\lambda=0.12793 \AA$ und der Probe-Detektor-Abstand $1415 \mathrm{~mm}$.

Der Phasenübergang begann bei einem Druck von 8 GPa und war bei 8.7 GPa abgeschlossen. Beim Entlasten der Probe in Schritten von 0.5 GPa wurde erst bei einem Druck von 1.4 GPa eine vollständige Rückumwandlung in die Niederdruckphase erreicht. Diese große Hysterese ist ein Indiz für eine hohe Aktivierungsenergie beim Phasenübergang.

Ein zweites im März/Juni 2013 durchgeführtes Experiment an CuInSe $e_{2}$ brachte keine auswertbaren Ergebnisse, da sich die Messtechnik an der Beamline P02.1 zu dieser Zeit noch in der Erprobung befand und noch keine passende Halterung für die Las1- und die 3Dia-Zelle existierte. Die improvisierte Halterung erwies sich nicht als ideal, sodass die Druckzelle während des Experiments verrutschte und somit nach wenigen $\omega$-Schritten keine Probenreflexe mehr gemessen wurden.

Für die Probe junjul12 besaßen Längs- und Querrichtung in der Ausgangsorientierung des Einkristalls keine niedrigen Indizes, die in Abbildung 7.19 angegebenen Richtungen sind Näherungen. Nach dem Phasenübergang wurde eine Schrumpfung der Probe um $8 \mu \mathrm{m}$ in Längsrichtung und $7 \mu \mathrm{m}$ in Querrichtung beobachtet. Dies entspricht einem jeweiligen Längenverlust von 7\%. Aufgrund der Druckzellengeometrie konnte die Metrikveränderung in der Normalenrichtung nicht gemessen werden. 

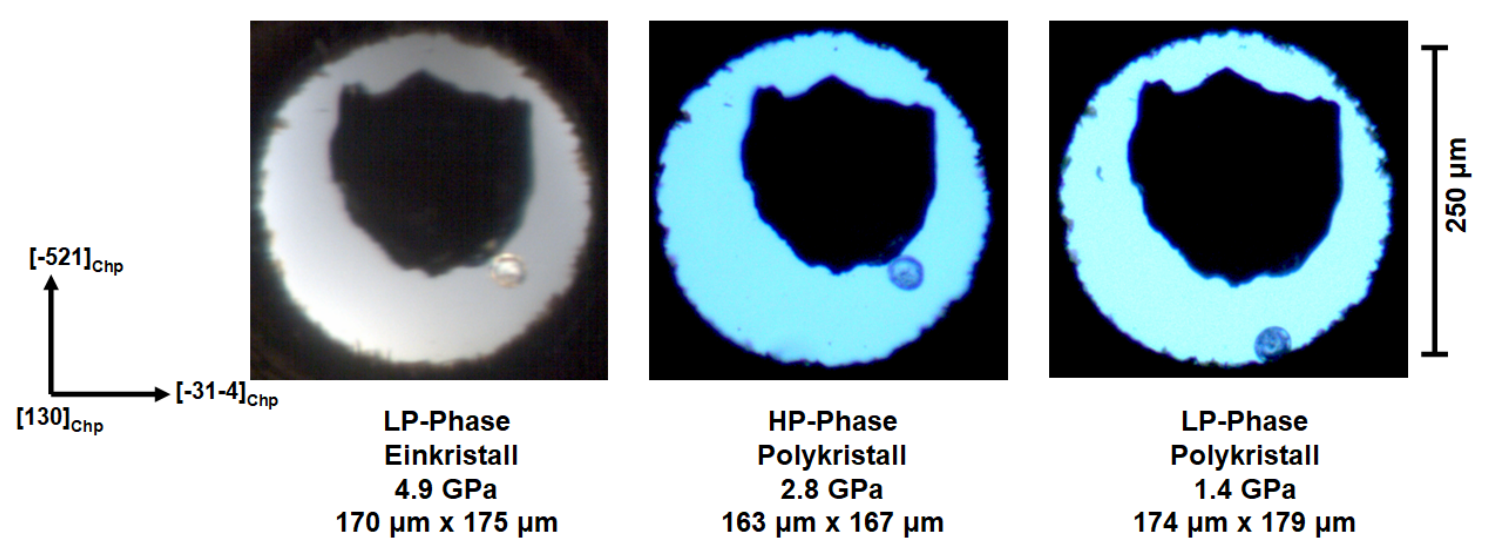

Abbildung 7.19: Aufnahmen der $\mathrm{CuInSe}_{2}$-Probe junjul12 während des Experiments. Links: Einkristall, Mitte: Polykristall in Hochdruckmodifikation, Rechts: Polykristall in Niederdruckmodifikation. Das Koordinatensystem auf der linken Seite zeigt die grobe Orientierung des Einkristalls zu Versuchsbeginn an.

Ves et al. [84] beobachteten beim Entlasten der Hochdruckphase einen Übergang in eine ungeordnete Struktur, Tinoco et al. [81] und Bovornratanaraks et al. [8] identifizierten die entlastete Niederdruckphase als Zinkblende-Typ. Ein Indiz für eine solche Rückumwandlung ist der 101-Reflex des Chalkopyrit-Typs, der beim Einkristall vorhanden, nach der Rückumwandlung in die Niederdruckphase jedoch nicht mehr sichtbar war (siehe Abbildung 7.20). Stattdessen ensprachen die beobachteten Reflexe im Experiment junjul12 denen des Zinkblende-Typs. Eine mögliche Erklärung für den Verlust des ChalkopyritTyps bei der Rückumwandlung ist das nichtstöchiometrische Verhältnis von $C u$ - und In-Kationen in der Probe und eine hohe Dichte von Gitterdefekten wie Leerstellen und Substitutionsdefekten [59], sodass beim Phasenübergang in die Hochdruckphase eine statistische Verteilung der Kationen stattfindet, weshalb der $\mathrm{NaCl}$-Typ erhalten wird, und diese statistische Verteilung der Kationen bei der Rückumwandlung in die Niederdruckphase erhalten bleibt.

Abbildung 7.21 zeigt die gemessenen Polfiguren der Probe junjul12. Längs-, Quer- und Normalenrichtung der Ausgangsorientierung des Einkristalls liegen nicht auf niedrig indizierten Richtungen (siehe auch Abbildung 7.19). Die Hochdruckphase im $\mathrm{NaCl-Typ}$ zeigt eine Orientierung, die der Orientierung des Einkristalls im Chalkopyrit-Typ entspricht. Die $\{112\}_{\mathrm{Chp}}$-Flächenpole des tetragonalen Einkristalls werden zu $\{111\}_{\mathrm{NaCl}^{-}}$ Flächenpolen der kubischen Hochdruckphase, ebenso wird der $\{001\}_{C h p}$-Flächenpol des Einkristalls zu einem der $\{100\}_{\mathrm{NaCl}^{-}}$-Flächenpole der Hochdruckphase. Die entlastete 

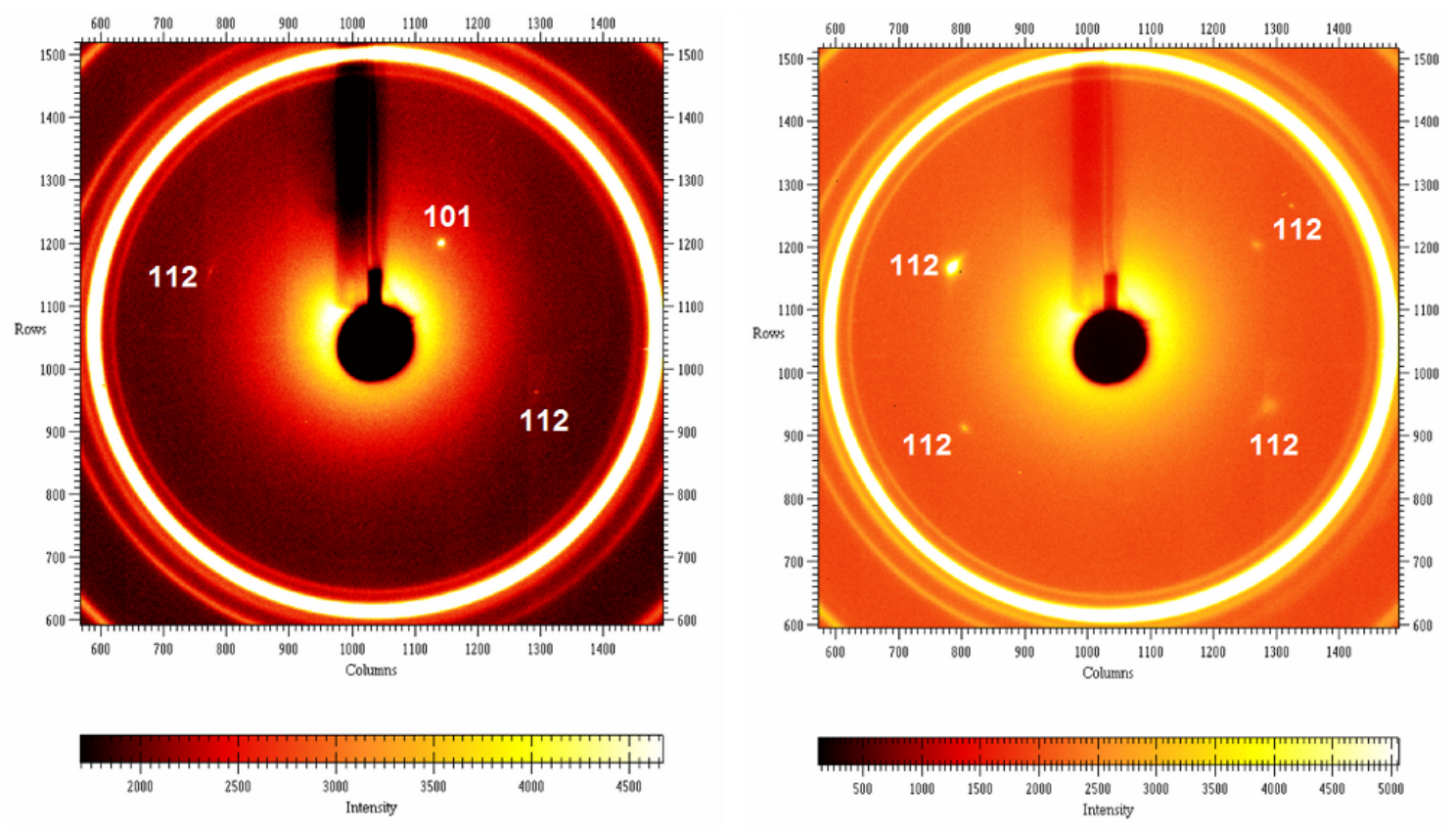

Abbildung 7.20: Beugungsbilder a) für den Einkristall im Chalkopyrit-Typ. Der 101-Reflex ist deutlich zu erkennen. b) für den Polykristall der Niederdruckphase. Der 101-Reflex ist nicht mehr zu erkennen. Beide Bilder wurden bei einem Winkel von $\omega=14^{\circ}$ unter gleicher Ausrichtung der Druckzelle aufgenommen. Der weiße Ring stellt den 110-Ferritreflex des Gaskets dar.

Niederdruckphase im Zinkblende-Typ weist wiederum die gleiche Orientierung wie Einkristall und Hochdruckphase auf. Die Verdoppelung mancher Flächenpole hat sich als systematischer Fehler bei der Messung der $90^{\circ}$-Polfiguren bei Hochdruck- und polykristaliner Niederdruckphase herausgestellt. Dies wurde dadurch bestätigt, dass bei $0^{\circ}$ - und $90^{\circ}$-Polfigur jeweils nur ein Flächenpol zu sehen ist und die Verdoppelung erst beim Zusammenfügen der beiden Polfiguren auftrat. Abbildung 7.22 zeigt die vollständige Lage aller niedrig indizierten Flächenpole für die Niederdruckphase im Chalkopyrit-Typ und

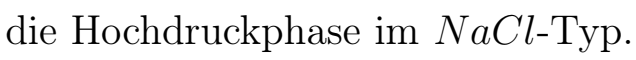

Abbildung 7.23 zeigt den Eulerraumschnitt der Hochdruckphase für $\varphi_{2}=20^{\circ}$. Es ist zu sehen, dass auch in der Hochdruckphase nur eine Orientierung bei $\varphi_{1}=38^{\circ}, \Phi=$ $15^{\circ}, \varphi_{2}=20^{\circ}$ existiert. Die lang gezogene Linse ist ein Artefaktreflex der Diamantstempel der Druckzelle (siehe auch Abbildung 6.3). Die ODF wurde mit kubischer Kristall- und trikliner Probensymmetrie berechnet. Der Reihenentwicklungsgrad betrug $\operatorname{Lmax}=22$, die Anzahl der verwendeten gemessenen Polfiguren 4. Für den Einkristall und die entlastete Niederdruckphase ließen sich aufgrund der vielen Artefaktreflexe der Druckzellenmaterialien und des Gaskets leider keine aussagekräftige ODF berechnen. 

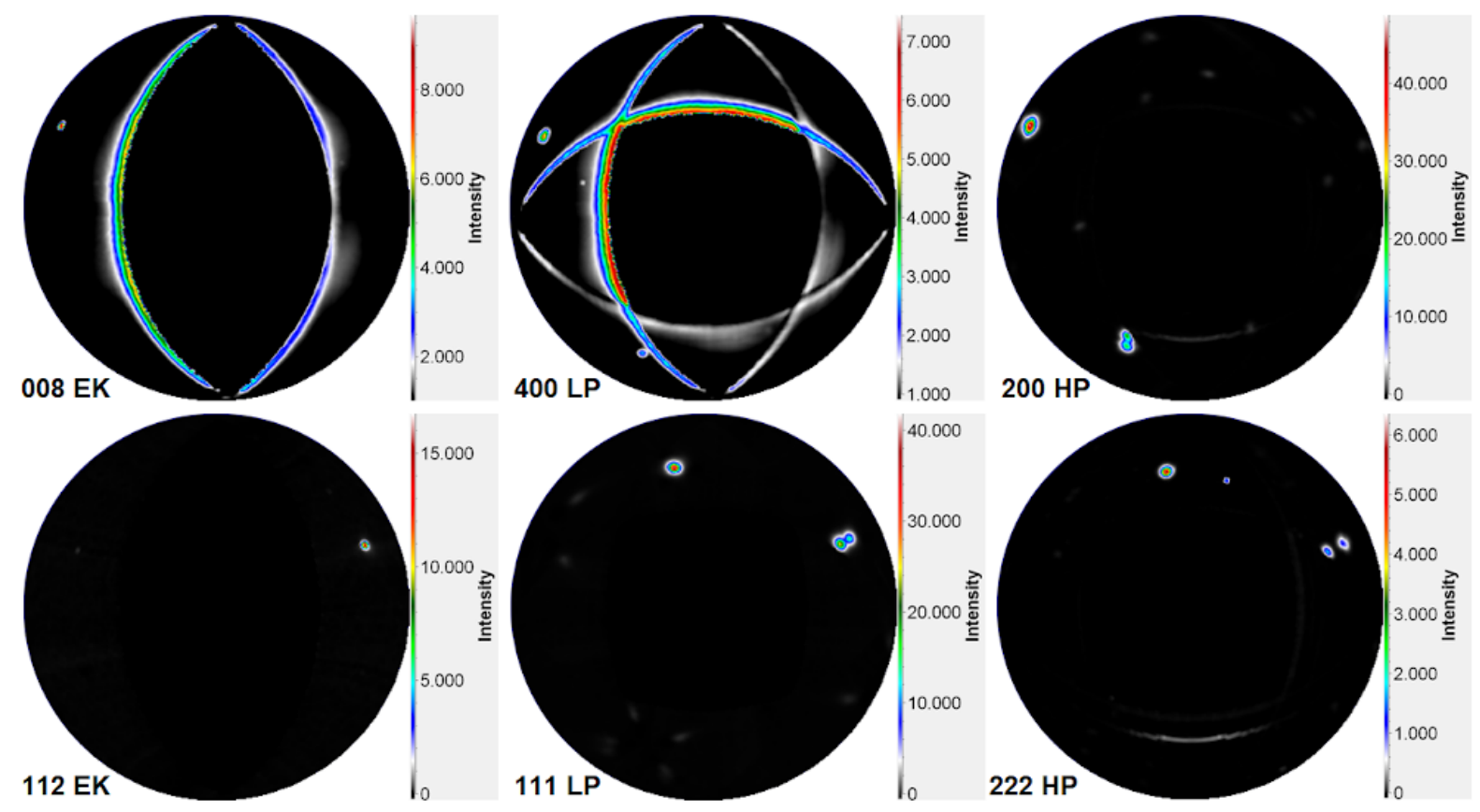

Abbildung 7.21: Ausgewählte gemessene Polfiguren für Hochdruck- und Niederdruckphase von $\mathrm{CuInSe} \mathrm{S}_{2}$. Die durchgehenden Bögen in der 008 EK- und der 400 LP-Polfigur entstammen der teilweisen Überlagerung der Probenreflexe mit dem 200-Ferritreflex des Gaskets.

Es ergeben sich die folgenden Orientierungsbeziehungen:

$$
[001]_{C h p} \|\langle 100\rangle_{N a C l}
$$

wobei zusätzlich gilt:

$$
\langle 100\rangle_{C h p} \|\langle 100\rangle_{N a C l}
$$

Da im kubischen Kristallsystem alle $\langle 100\rangle$-Richtungen symmetrisch äquivalent sind, ergibt sich aus den Orientierungsbeziehungen unter anderem folgende mögliche Achsentransformation:

$$
\begin{aligned}
\vec{a}_{N a C l} & =\vec{a}_{C h p} \\
\vec{b}_{N a C l} & =\vec{b}_{C h p} \\
\vec{c}_{N a C l} & =\vec{c}_{C h p}
\end{aligned}
$$



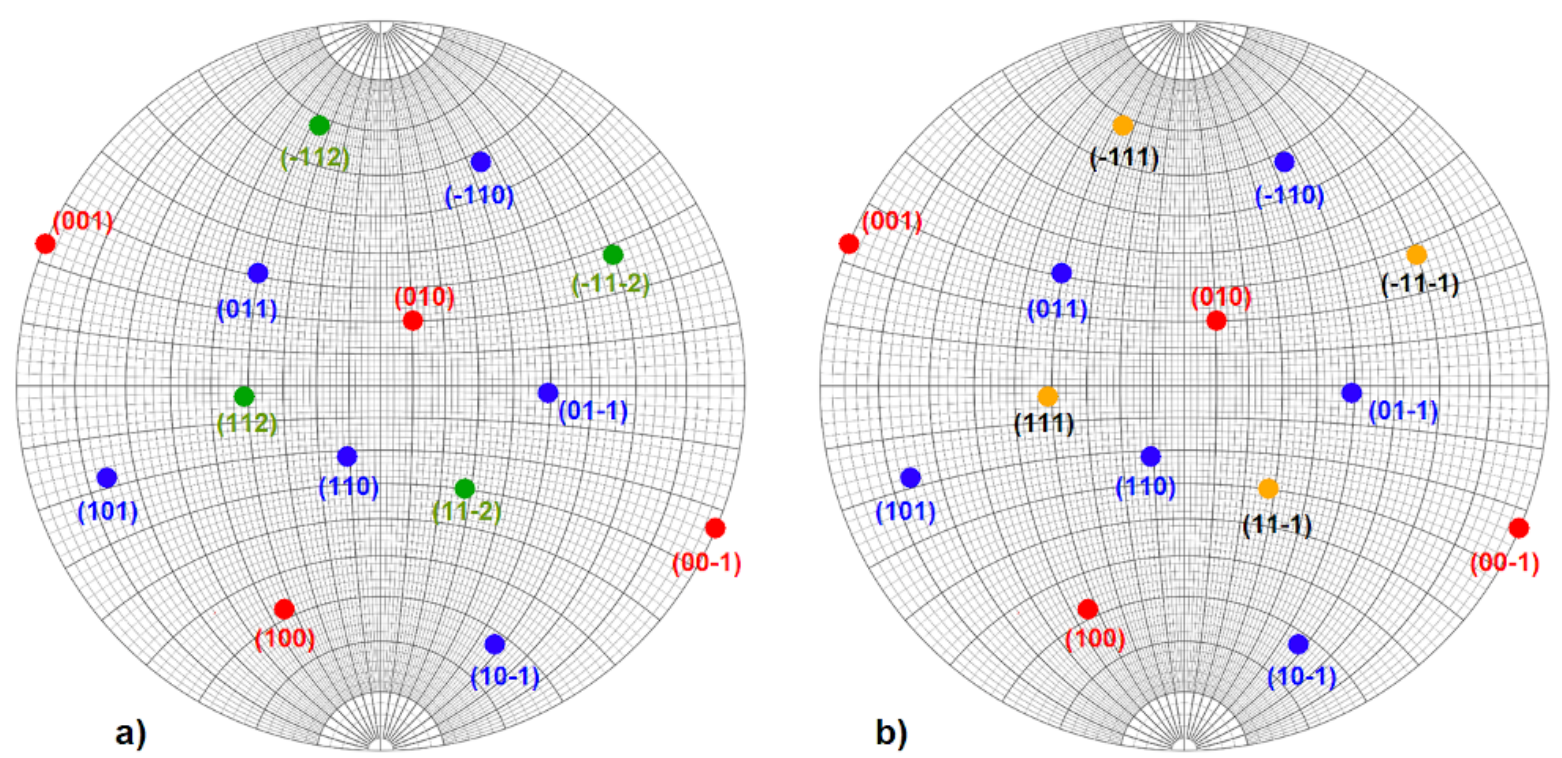

Abbildung 7.22: Orientierungsbeziehungen zwischen a) Niederdruckphase im Chalkopyrit-Typ und b) Hochdruckphase im $\mathrm{NaCl}$-Typ von $\mathrm{CuInSe}_{2} .\{100\}$-Flächenpole des Chalkopyrit-Typs und $\mathrm{NaCl}$-Typs sind in rot, $\{110\}$-Flächenpole des Chalkopyrit-Typs und $\mathrm{NaCl}$-Typs in blau, $\{112\}$-Flächenpole des Chalkopyrit-Typs in grün und $\{111\}$-Flächenpole des $\mathrm{NaCl}$-Typs in gelb dargestellt. Die Orientierung der Phasen entspricht der Orientierung der Probe.

mit der Transformationsmatrix

$$
P=\left(\begin{array}{lll}
1 & 0 & 0 \\
0 & 1 & 0 \\
0 & 0 & 1
\end{array}\right)
$$

Die Raumgruppe des Chalkopyrittyps $I \overline{4} 2 d$ ist eine Untergruppe des Zinkblende-Typs F $\overline{4} 3 m$. Kationen und Anionen im Chalkopyrit-Typ sitzen auf den gleichen Positionen wie im Zinkblende-Typ, aufgrund der abwechselnden Besetzung der Tetraederlücken mit

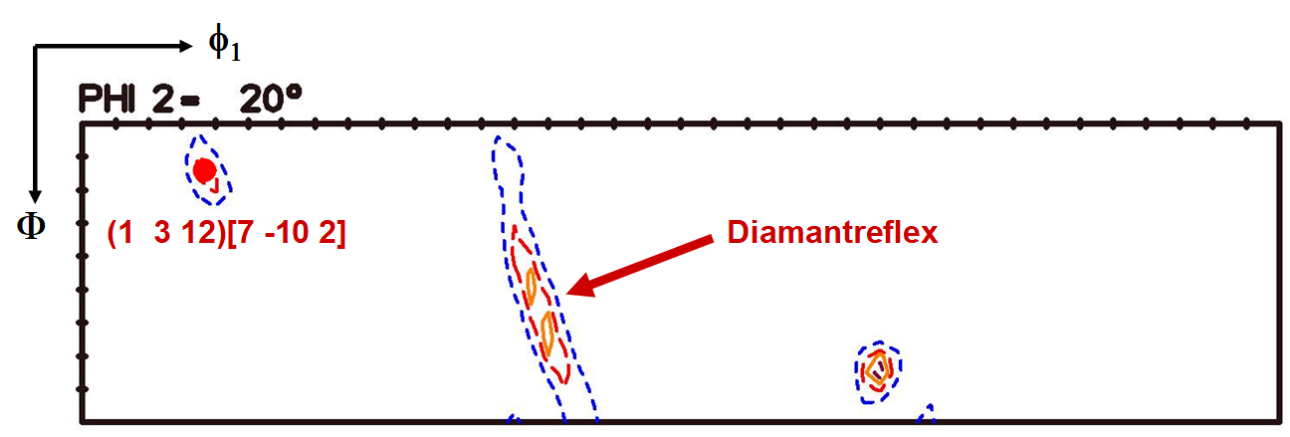

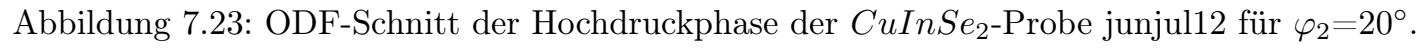


zwei verschiedenen Kationen verdoppelt sich die Länge der c-Achse der Elementarzelle. Deshalb wurden bei der Suche nach einem Transformationspfad, der die erhaltenen Orientierungsbeziehungen erklärt, Transformationspfade vom Zinkblende-Typ zum $\mathrm{NaCl}$ Typ in Betracht gezogen.

Capillas et al. [16] schlagen mehrere Transformationspfade vom Zinkblende-Typ in den $\mathrm{NaCl}$-Typ vor, in denen die $\langle 100\rangle$-Richtungen der Elementarzelle erhalten bleiben. Der Transformationspfad über die gemeinsame Untergruppe $R 3 m$ hat die Achsentransformationen $a_{Z n S}=a_{N a C l}, b_{Z n S}=b_{N a C l}, c_{Z n S}=c_{N a C l}$, genau wie der Transformationspfad über die gemeinsame Untergruppe $P 2_{1} 3$. Der Transformationspfad über die gemeinsame Untergruppe $P m n 2_{1}$ erfordert eine Achsentransformation $a_{Z n S}=a_{N a C l}, b_{Z n S}=b_{N a C l}$, $c_{Z n S}=c_{N a C l}$ und eine Ursprungsverschiebung um $\left(\frac{1}{4}, 0,-\frac{1}{4}\right)$. Für den Transformationspfad über die gemeinsame Untergruppe $P 2_{1}$ lauten die Achsentransformationen $a_{Z n S}=-a_{N a C l}, b_{Z n S}=c_{N a C l}, c_{Z n S}=b_{N a C l}$, der Ursprung wird um $\left(-\frac{1}{4}, 0,-\frac{1}{4}\right)$ verschoben. Die von Capillas et al. für diese vier Transformationspfade berechnete Gitterspannung ist für alle Pfade gleich hoch, die vier Transformationspfade unterscheiden sich allerdings in ihren ebenfalls von Capillas et al. berechneten maximalen Atomverschiebungen. Für die Transformationspfade über $R 3 m$ und $P 2_{1} 3$ beträgt die maximale Atomverschiebung $1.7208 \AA$, für die Transformationspfade über $P m n 2_{1}$ und $P 2_{1}$ nur $1.4050 \AA$. Diese Werte gelten für $S i C$ mit einem Gitterparameter $a_{Z n S}=3.974 \AA$ für

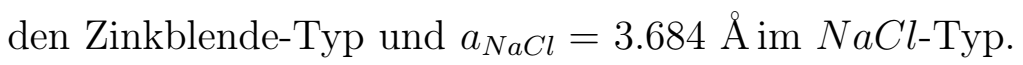

Unter den zehn Transformationspfaden, deren Aktivierungsenergien von Hatch et al. [30] berechnet wurden, weist lediglich der auch schon von Capillas et al. erwähnte Transformationspfad über die gemeinsame Untergruppe $P 2_{1} 3$ die erforderlichen Achsentransformationen auf, hier in der Variante $a_{Z n S}=-b_{N a C l}, b_{Z n S}=-c_{N a C l}, c_{Z n S}=a_{N a C l}$. Die berechnete Aktivierungsenergie dieses Pfades liegt fast vier mal so hoch wie die berechnete Aktivierungsenergie der acht energetisch günstigsten Transformationspfade vom Zinkblende- zum $\mathrm{NaCl-Typ.}$

Da aufgrund der kubischen Elementarzellensymmetrie der Hochdruckphase und der entlasteten Niederdruckphase die Hauptrichtungen der Elementarzelle symmetrisch äquivalent sind und auch eine Verschiebung des Ursprungs mit den vorhandenen Auswertungsmethoden nicht erkannt werden kann, kann an dieser Stelle keine eindeutige Aussage über einen Transformationspfad gemacht werden. 


\subsection{Nickelsulfid (NiS)}

Am Nickelsulfid wurden bereits röntgenographische Studien zum Verhalten unter extremen Bedingungen durchgeführt: Sowa, Ahsbahs und Schmitz [75] untersuchten das Hochdruckverhalten eines Millerit-Einkristalls bei Raumtemperatur und Drücken von bis zu 26.8 GPa, ohne einen Phasenübergang auszulösen. Unter Hochdruck- und Hochtemperaturbedingungen fanden sie für NiS-Pulver einen Phasenübergang vom Millerit-Typ in den NiAs-Typ und stellten eine starke Abhängigkeit der Umwandlungstemperatur vom Druck fest.

Sowa, Klein und Raue [77] untersuchten die Orientierungsbeziehungen zwischen MilleritTyp und NiAs-Typ für NiS mit Hilfe von Synchrotronstrahlung. Sie berechneten die Polfiguren der Hochtemperaturphase mit MAUD, die Orientierung des Einkristalls wurde aus den Flächendetektorbildern hergeleitet.

Am Nickelsulfid wurde ein erfolgreiches Experiment im August 2007 an der Beamline BW5 im Glaskugelofen durchgeführt. Zuerst wurde der mit der [001]-Richtung parallel zur $\omega$-Achse ausgerichtete Millerit-Einkristall bei Raumtemperatur in einem Winkelbereich $-27^{\circ} \leq \omega \leq 93^{\circ}$ gemessen. Anschließend wurde die Probe langsam auf $400^{\circ} \mathrm{C}$ erhitzt, wobei eine Umwandlung in den NiAs-Typ mit Resten des Millerit-Typs stattfand, wieder auf Raumtemperatur abgekühlt, und in einem Winkelbereich $-87^{\circ} \leq \omega \leq 93^{\circ}$ gemessen. In der abgekühlten polykristallinen Probe existieren Millerit-Typ und NiAsTyp nebeneinander, wobei der Anteil des NiAs-Typs überwiegt. Der Probe-DetektorAbstand betrug bei allen Messungen $1300 \mathrm{~mm}$, die Schrittweite $\Delta \omega=1^{\circ}$ und die Wellenlänge $\lambda=0.11944 \AA$.

Eine zweites, im Dezember 2008 durchgeführtes Experiment ließ sich leider nicht auswerten, weil der Probenkristall während des Aufheizens verrutschte und somit keine Orientierungsbeziehungen mehr bestimmt werden konnten. Beim Erhitzen der Probe wurde eine fast vollständige Umwandlung in den NiAs-Typ mit geringen Resten des Millerit-Typs erreicht, die auch nach dem Abkühlen der Probe erhalten blieb. Nach anschließendem weiterem mehrfachen Aufheizen und wieder Abkühlen zeigte die Probe keine Spuren des Millerit-Typs mehr.

Um die mit MAUD berechneten Polfiguren der Hochtemperaturphase und die mit dem 

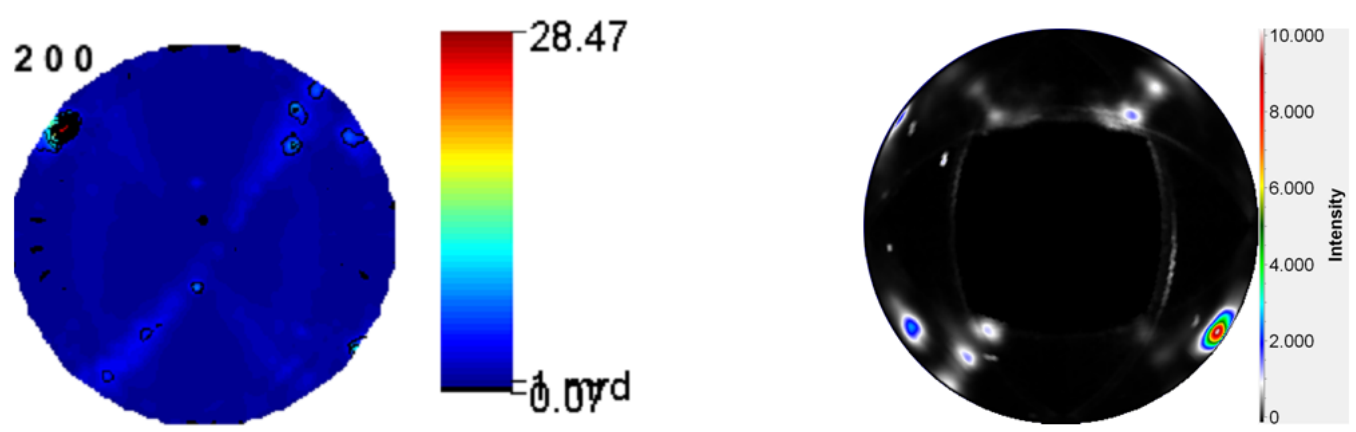

\section{Rotation um $180^{\circ}$ um die Normalenrichtung}

Abbildung 7.24: Vergleich der Orientierungsdarstellung der Polfiguren in MAUD (links) und im PoleFigureInterpolator (rechts) am Beispiel der $\{100\}_{\mathrm{NaCl}^{-}}$Polfigur von InAs aus dem Experiment dez11. Die in MAUD erstellten Polfiguren sind im Vergleich zu den Polfiguren aus dem PoleFigureInterplator um $180^{\circ}$ um die Normalenrichtung gedreht.

PoleFigureInterpolator erstellten Polfiguren der Tieftemperaturphase miteinander in Relation setzen zu können, wurde die Orientierung der sowohl mit MAUD als auch mit dem PoleFigureInterpolator erstellten $\{100\}_{\mathrm{NaCl}}$-Polfigur von InAs aus dem Experiment dez11 miteinander verglichen (siehe Abbildung 7.24). Hierbei stellte sich heraus, dass die im PoleFigureInterpolator erstellte Polfigur um $180^{\circ}$ um die Normalenrichtung gedreht werden musste, um mit der Polfigur aus MAUD deckungsgleich zu werden. Dies wurde bei der Auswertung der Daten des Experiments berücksichtigt.

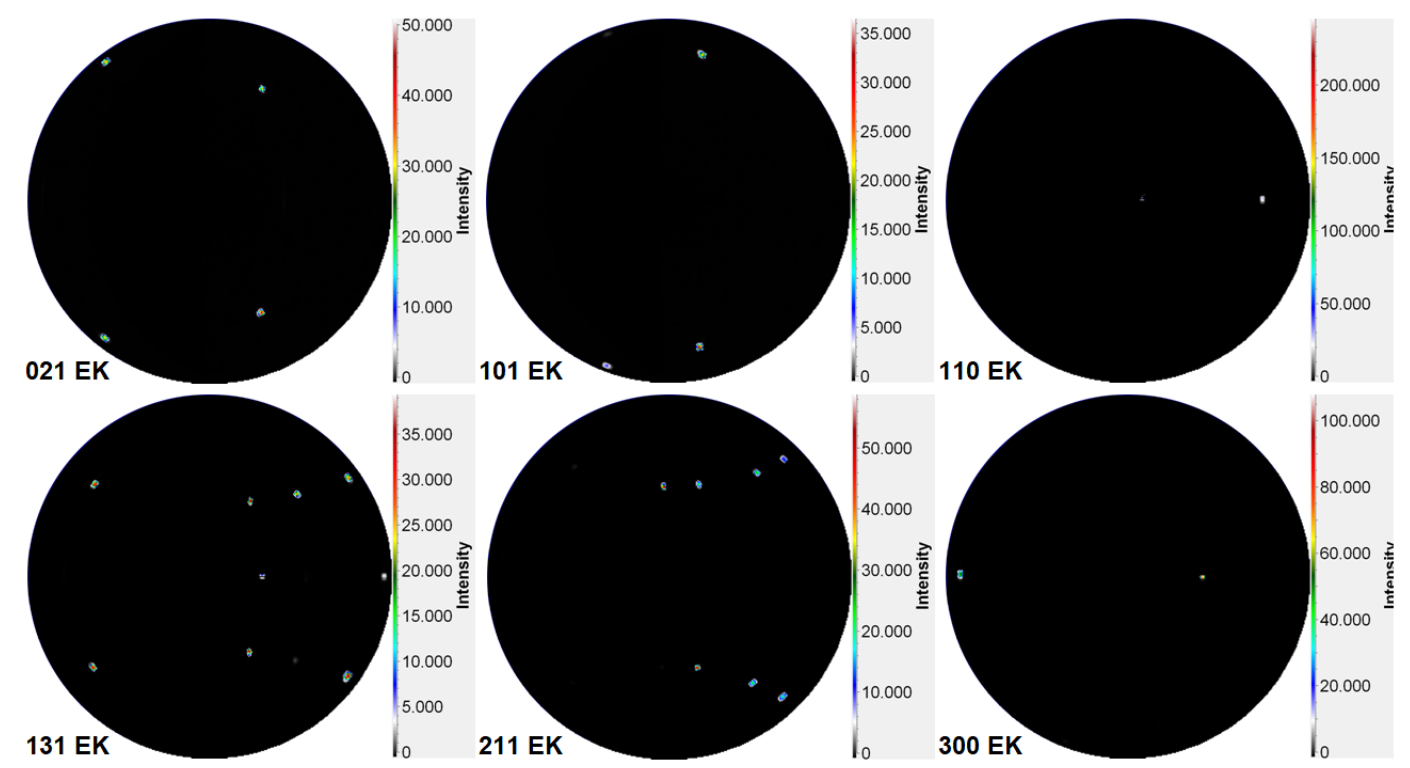

Abbildung 7.25: Ausgewählte Polfiguren des NiS-Einkristalls aus der Messung aug07. 


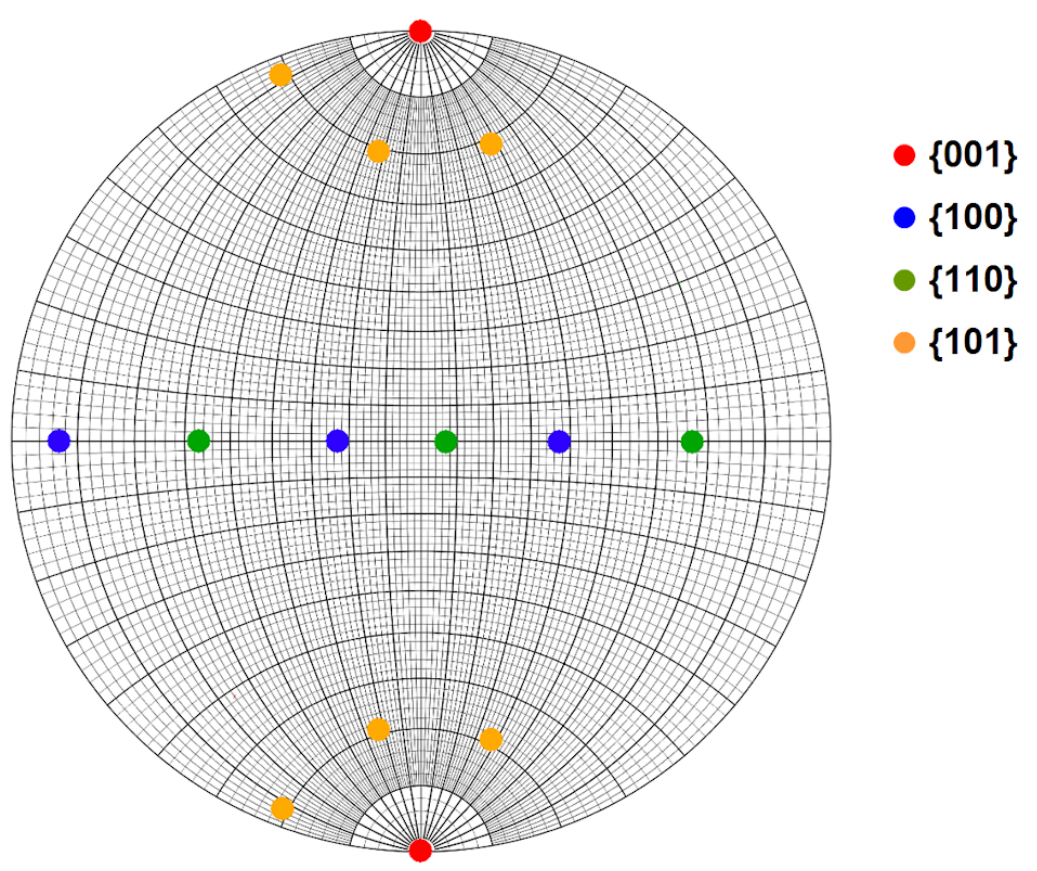

Abbildung 7.26: Orientierung des NiS-Einkristalls aus dem Experiment aug07.

Die gemessenen Polfiguren des NiS-Experiments aug07 für den Einkristall in der Tieftemperaturphase zeigen eine Orientierung $[001]_{\text {Millerit }}$ parallel zur Längsrichtung (siehe Abbildung 7.25). Alle gemessenen Polfiguren wurden gemäß Abbildung 7.24 um 180 um die Normalenrichtung gedreht. Abbildung 7.26 zeigt die aus den gemessenen Polfiguren extrapolierte vollständige Orientierung des Einkristalls im Wulffschen Netz mit $[001]_{\text {Millerit }}$ parallel zur Längsrichtung. Der Flächenpol einer der $\{110\}_{\text {Millerit }}$-Netzebenen ist um etwa $7^{\circ}$ in Querrichtung gegen die Normalenrichtung verkippt. Das sehr kleine $c / a$-Achsenverhältnis der Elementarzelle des Millerits von $c / a=0.327$ lässt sich daran erkennen, dass die $\{101\}_{\text {Millerit }}$-Flächenpole sehr nahe an der $[001]_{\text {Millerit }}$-Richtung liegen.

Die mit MAUD berechneten Polfiguren des NiS-Experiments aug07 für die Hochtemperaturphase im NiAs-Typ zeigen eine Orientierung $[001]_{N i A s}$ parallel zur Längsrichtung. Somit ergibt sich folgende Orientierungsbeziehung:

$$
[001]_{\text {Millerit }} \|[001]_{N i A s}
$$

Alle Polfiguren außer der $\{001\}_{N i A s}$-Polfigur zeigen als Orientierungsverteilung Gürtel mit Maxima alle $60^{\circ}$ rotationssymmetrisch um die Längsrichtung. Für die $\{100\}_{N i A s}{ }^{-}$ 


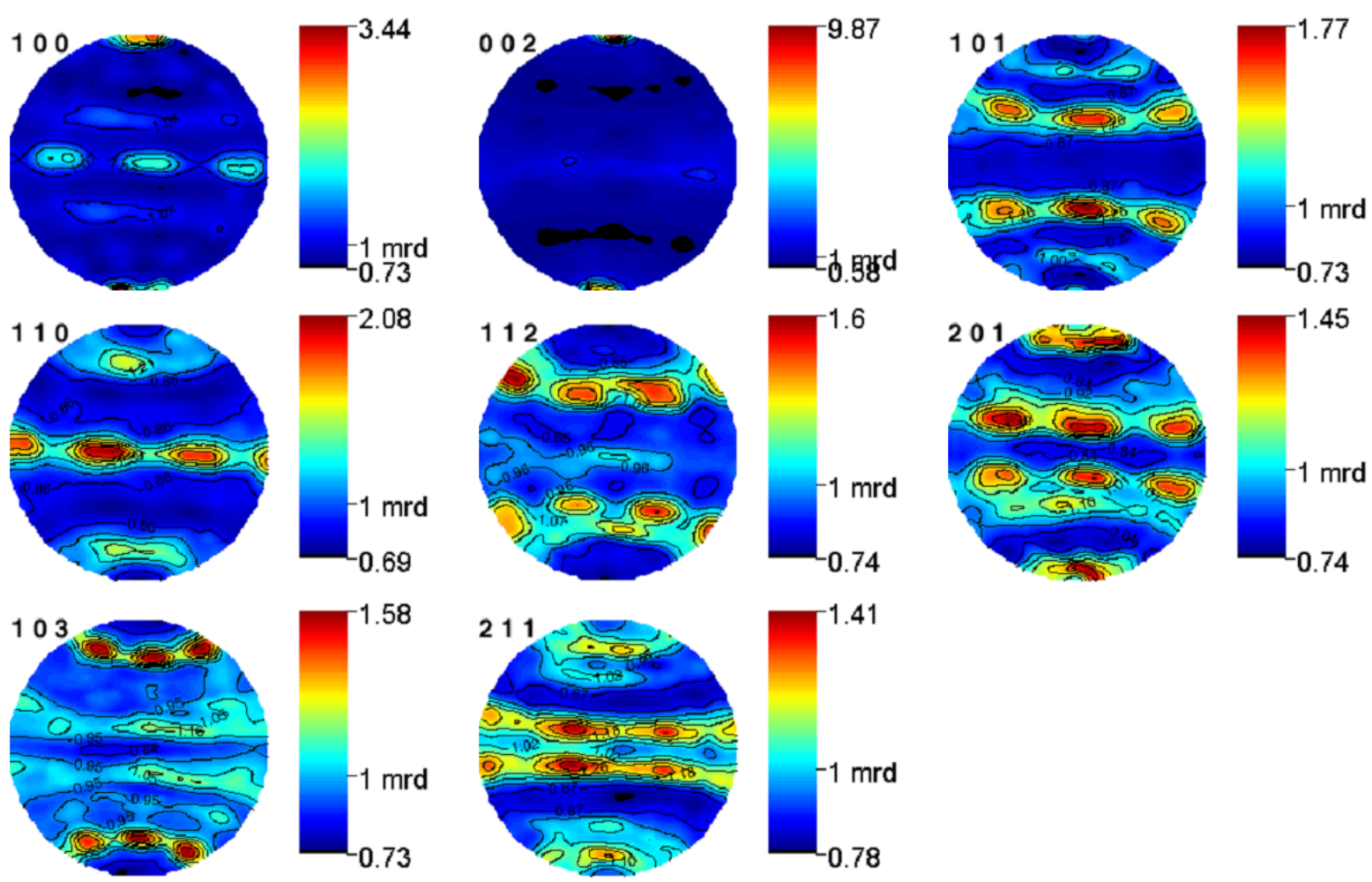

Abbildung 7.27: Ausgewählte mit MAUD berechnete vollständige Polfiguren der Hochtemperaturphase von $N i S$ aus dem Experiment aug07.

Polfigur liegt eines dieser Maxima leicht von der Normalenrichtung aus in Querrichtung ausgelenkt, analog zu einem der $\{110\}_{\text {Millerit }}{ }^{-F l a ̈ c h e n p o l e . ~ D i e s ~ f u ̈ h r t ~ z u ~ f o l g e n d e r ~ O r i-~}$ entierungsbeziehung:

$$
[110]_{\text {Millerit }} \|[210]_{N i A s}
$$

Da [110]-Richtung und [100]-Richtung im trigonalen und hexagonalen Kristallsystem symmetrisch äquivalent sind, gilt somit für die Orientierungsbeziehungen:

$$
\begin{aligned}
& {[100]_{\text {Millerit }} \|[210]_{\text {NiAs }}} \\
& {[210]_{\text {Millerit }} \|[100]_{\text {NiAs }}}
\end{aligned}
$$

Dies entspricht einer Rotation der Einheitsvektoren der Elementarzelle um $30^{\circ}$ um die gemeinsame [001]-Richtung von Tieftemperatur- und Hochtemperaturphase.

Diese Orientierungsbeziehungen wurden auch von Sowa et al. [77] beobachtet. 


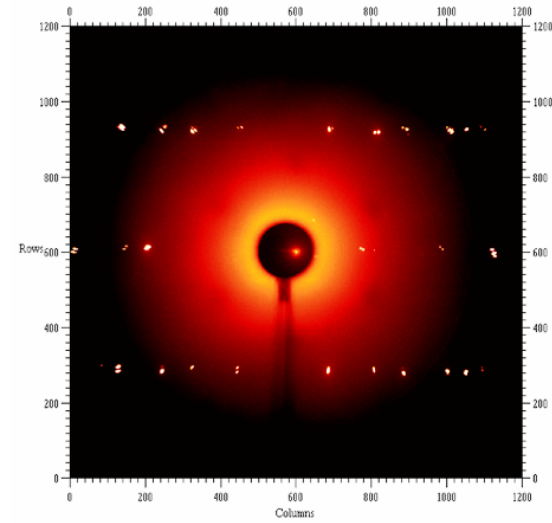

a)

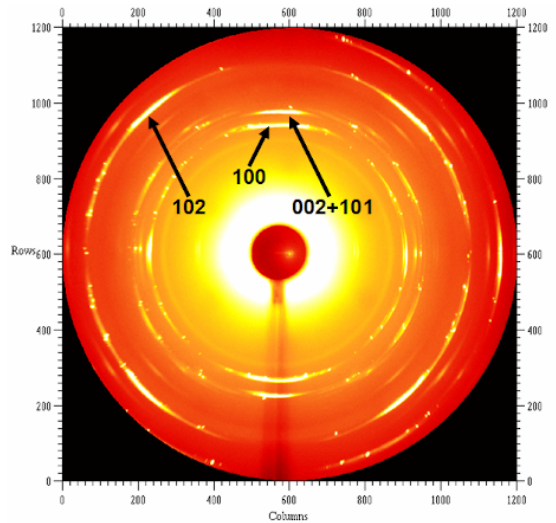

b)

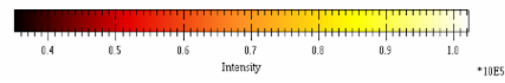

Abbildung 7.28: Aufsummierte Detektorbilder a) des Einkristalls im Millerit-Typ, b) der polykristallinen Hochtemperaturphase im NiAs-Typ mit Resten der Tieftemperaturphase im Millerit-Typ. Die Reflexe der Hochtemperaturphase sind mit schwarzen Pfeilen markiert und indiziert.

In einigen der berechneten Polfiguren sind zusätzlich zur Hauptorientierung weitere, schwächere Orientierungen zu sehen. Diese sind wahrscheinlich Artefakte der Reste der Tieftemperaturphase, die mehrere Reflexüberlagerungen mit der Hochtemperaturphase

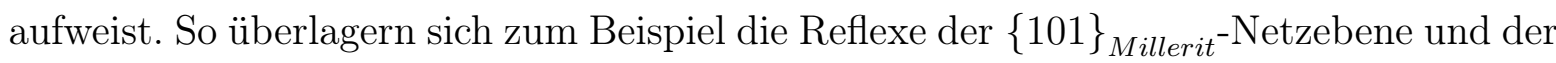

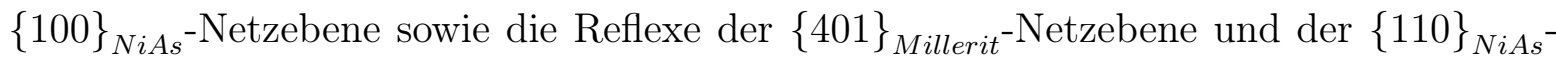
Netzebene. Außerdem wurden über den ganzen Ring verteilt körnige Einzelreflexe in den Reflexringen der Hochtemperaturphase beobachtet, die die berechneten Polfiguren ebenfalls verfälschen können (siehe Abbildung 7.28).

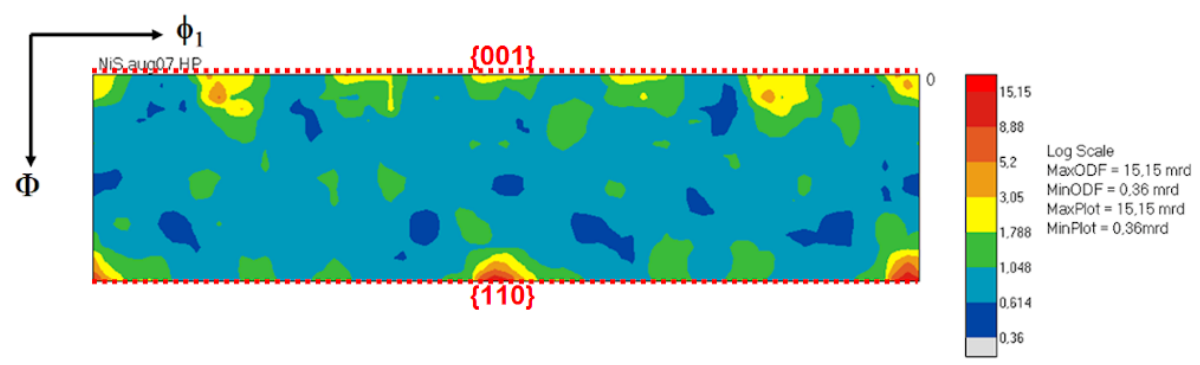

Abbildung 7.29: Mit BEARTEX [88] aus den vollständigen Polfiguren berechneter $\varphi_{2}=0^{\circ}$-Schnitt des Eulerraums für Hochtemperaturphase von NiS aus dem Experiment aug07. Die $\{001\}$ und $\{110\}$-Fasern sind als rote gestrichelte Linien eingezeichnet.

Abbildung 7.29 zeigt den ODF-Schnitt für $\varphi_{2}=0^{\circ}$ für die Hochtemperaturphase der NiSProbe aug07. Die ODF wurde aus den von MAUD ausgegebenen Polfiguren mit BEAR- 
TEX [88] berechnet. Die Berechnung wurde mit trigonaler Proben- und hexagonaler Kristallsymmetrie durchgeführt. Die sechszählige Symmetrie der Orientierungen entlang der $\{001\}$-Faser ist deutlich zu sehen. Die beiden Haupt-Orientierungsmaxima liegen auf einer $\{110\}$-Faser so gut wie in idealer Lage. Diese Orientierungsverteilung deckt sich mit den Beobachtungen aus der manuellen Auswertung der Polfiguren. Für den Einkristall der Tieftemperaturphase konnte keine sinnvolle ODF berechnet werden, da bei der Erstellung der berechneten vollständigen Polfiguren immer wieder Reflexe verschwanden oder Geisterreflexe auftraten.
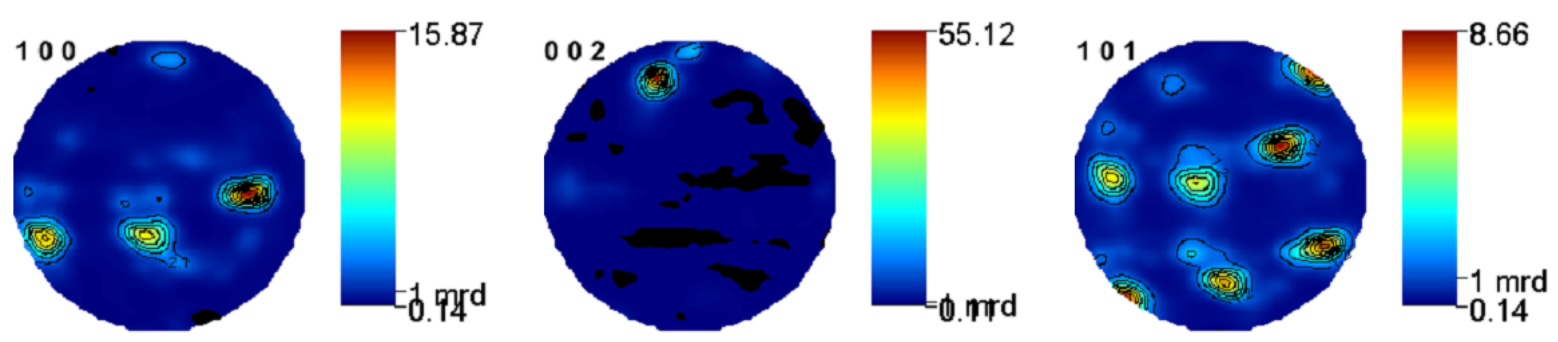

Abbildung 7.30: Ausgewählte mit MAUD berechnete Polfiguren der Hochtemperaturphase des Experiments dez08. Die Verkippung der Probe gegen die $\omega$-Achse des Goniometers ist deutlich zu sehen.

Abbildung 7.30 zeigt ausgewählte Polfiguren der Hochtemperaturphase des NiS-Experiments dez08. Auch wenn sich die Orientierungsbeziehungen aufgrund der Verkippung der Probe während des Aufheizens nicht auswerten lassen, ist zu erkennen, dass diese Probe die gleichen Orientierungen in der Hochtemperaturphase aufweist wie die Probe des Experiments aug07. Die Textur ist wesentlich schärfer als im Experiment aug07 und es gibt keinen deutlichen Untergrund, was auf die Abwesenheit von Artefaktreflexen schließen lässt.

Aus den Orientierungsbeziehungen zwischen Tief- und Hochtemperaturphase von NiS konnte kein Transformationspfad direkt bestimmt werden. Ebenso konnte nicht geklärt werden, welche atomaren Verschiebungen mit der Transformation einher gehen, die zu den erhaltenen Orientierungsbeziehungen führen. Es kommen nur Transformationspfade in Frage, die die [001]-Richtung intakt lassen und eine $30^{\circ}$-Rotation der Elementarzellenvektoren um die gemeinsame [001]-Richtung von Tieftemperatur- und Hochtemperaturphase enthalten. Die c-Achse der Elementarzelle verlängert sich während des Phasenübergangs von $c_{\text {Millerit }}=3.1434 \AA$ auf $c_{N i A s}=5.3514 \AA$, was darauf hindeuten kann, dass durch den Phasenübergang eine Verdoppelung der Elementarzelle in [001]-Richtung stattfindet. 
Eine gemeinsame Untergruppe eines Transformationspfads müsste einer Raumgruppe der Punktgruppen 3m, 3, $m$ oder 1 angehören. 


\section{Zusammenfassung und Ausblick}

Die vorliegende Arbeit hat gezeigt, dass es trotz vieler Schwierigkeiten bei Messung und Auswertung prinzipiell möglich ist, Orientierungsbeziehungen zwischen verschiedenen Phasen einer Substanz mit Hilfe von Synchrotronstrahlung zu bestimmen und daraus potentielle Mechanismen für den Phasenübergang abzuleiten. So konnte die ursprünglich geplante Auswertung der Daten mit dem Programm MAUD nur in Ausnahmefällen erfolgreich durchgeführt werden, weshalb die Auswertung größtenteils händisch erfolgte. Für den druckinduzierten Phasenübergang in Indiumarsenid konnte zwar kein Transformationspfad über eine gemeinsame Untergruppe gefunden werden, jedoch konnten die Orientierungsbeziehungen zwischen Hoch- und Niederdruckphase bestimmt und daraus der wahrscheinliche atomare Mechanismus der Transformation abgeleitet werden. Die Orientierungsbeziehungen zwischen Hochdruck- und Niederdruckphase von Kupferindiumdiselenid wiesen auf eine Reihe möglicher Transformationspfade hin. Für den druckinduzierten Phasenübergang in Cadmiumsulfid und Cadmiumselenid konnten vermutete Orientierungsbeziehungen und ein vermuteter Transformationspfad bestätigt werden. Für den temperaturinduzierte Phasenübergang in Nickelsulfid konnten ebenfalls bereits angenommene Orientierungsbeziehungen bestätigt werden, wenn auch kein Transformationspfad.

Ein oft auftretendes Problem bei den Hochdruckmessungen war die Überlagerung der Probenreflexe mit den Reflexen des Stahlgaskets und der Diamantstempel. Die Überlagerung durch Gasketreflexe ließe sich verringern, wenn das Gasketmaterial so gewählt wird, dass möglichst wenige Probenreflexe überdeckt werden. Eine Alternative zu Stahlgaskets wären z.B. Wolframgaskets. Bei Materialien mit hexagonalem Kristallsystem war es oft schwierig, die innersten, sehr eng beieinander liegenden Probenreflexe sauber voneinander zu trennen. Hier würde es sich anbieten, den Probe-Detektor-Abstand zu vergrößern um eine bessere Reflextrennung zu erreichen. Die vorhandenen Auswer-

tungsprogramme ließen sich, wenn überhaupt, nur unterstützend für die Auswertung der Probendaten verwenden, da sie ursprünglich für andere Zwecke entworfen wurden. 
Hier könnte die Entwicklung eines Auswertungsprogramms, das auch scharfe Texturen und schwache Reflexintensitäten erkennen kann, Abhilfe schaffen. Zur Bestimmung von Phasenübergangsmechanismen wäre eine Kombination aus experimentellen Methoden und computergestützter Modellierung von Phasenübergängen interessant. 


\section{Literaturverzeichnis}

[1] The mar345 user's guide www.marxperts.com/man/mar345/index.html.

[2] G. J. Ackland. High-pressure phases of group IV and III-V semiconductors. Reports on Progress in Physics, 64:483-516, 2001.

[3] H. Ahsbahs. 20 Jahre Merrill-Bassett-Zelle. Einige Neuheiten. Zeitschrift für Kristallographie, Suppl., 9:42, 1995.

[4] H. Ahsbahs. New pressure cell for single-crystal X-ray investigations on diffractometers with area detectors. Zeitschrift für Kristallographie, 219:305-308, 2004.

[5] J. Als-Nielsen and D. McMorrow. Elements of Modern X-Ray Physics. John Wiley and Sons, Ltd., 2 edition, 2011.

[6] B. Batlogg, A. Jayaraman, J. E. Van Cleve, and R. G. Maines. Optical absorption, resistivity, and phase transformation in CdS at high pressure. Physical Review, B27:3920-3923, 1983.

[7] M. A. Blanco, J. M. Recio, A. Costales, and R. Pandey. Transition path for the $B 3 \rightleftharpoons B 1$ phase transformation in semiconductors. Physical Review B, 62:R10599$602,2000$.

[8] T. Bovornratanaraks, V. Saengsuwan, K. Yoodee, M. I. McMahon, C. Hejny, and D. Ruffolo. High pressure orthorhombic structure of CuInSe $e_{2}$. Journal of Physics: Condensed Matter, 22:1-4, 2010.

[9] W. H. Bragg. The Reflection of X-Rays by Crystals (II). Proceedings of the Royal Society of London, A 89:240, 1913. 
[10] H. Bärninghausen. Group-subgroup relations between space groups: a useful tool in crystal chemistry. MATCH Commun. Math. Comput. Chem., 9:139-175, 1980.

[11] M. Buerger. Crystallographic Aspects of Phase Transformations. Phase transformation in solids, edited by R. Smoluchowski, J. E. Mayers, and W. A. Weyl, John Wiley, New York, 1:183-211, 1951.

[12] M. J. Buerger. Polymorphism and phase transformations. Fortschr. Mineral., 29:9$24,1961$.

[13] H.-J. Bunge. Mathematische Methoden der Texturanalyse. Akademie-Verlag, Berlin, 1 edition, 1969.

[14] H.-J. Bunge. Texture Analysis in Materials Science. Cuvillier Verlag Göttingen, 1 edition, 1993.

[15] H. J. Bunge and H. Klein. Determination of Quantitative, High-resolution Pole Figures with the Area Detector. Zeitschrift für Metallkunde, 87:465-472, 1996.

[16] C. Capillas, J. M. Perez-Mato, and M. I. Aroyo. Maximal symmetry paths for reconstructive phase transitions. Journal of Physics: Condensed Matter, 19:275203, 2007.

[17] M. Catti. Orthorhombic intermediate state in the zinc blende to rocksalt transformation path of SiC at high pressure. Physical Review Letters, 87:35504, 2001.

[18] A. G. Christy. Multistage Diffusionless Pathways for Reconstructive Phase Transitions: Application to Binary Compounds and Calcium Carbonate. Acta Crystallographica $B, 49: 987-996,1993$.

[19] J. A. Corll. Effect of Pressure on the Elastic Parameters and Structure of CdS. Physical Review, 157:623-626, 1967.

[20] A-C. Dippel, H-P. Liermann, J. Delitz, P. Walter, H. Schulte-Schrepping, O. Seeck, and H. Franz. Beamline P02.1 at PETRA III for high-resolution and high-energy powder diffraction. Journal of Synchrotron Radiation, 22:675-687, 2015. 
[21] V. P. Dmitriev, S. B. Rochal, Yu. M. Gufan, and P. Toledano. Definition of a Transcendental Order Parameter for Reconstructive Phase Transitions. Physical Review Letters, 60:1958-1961, 1988.

[22] V. P. Dmitriev and P. Toledano. Reconstructive Phase Transitions In Crystals and Quasicrystals. World Scientific Publishing Co Pte Ltd, 1 edition, 1996.

[23] P. Ehrenfest. Phasenumwandlungen im ueblichen und erweiterten Sinn, classifiziert nach den entsprechenden Singularitaeten des thermodynamischen Potentiales. Koninkl. Akad. Wetenschap. Amsterdam Proc., 36:153-157, 1933.

[24] G. Gisecke and H. Pfister. Präzisionsbestimmungen der Gitterkonstanten von $A^{I I I}$ $B^{V}$-Verbindungen. Acta Crystallographica, 11:369-371, 1958.

[25] J. González, M. Quintero, and C. Rincón. Pressure dependence of the Raman A1 mode and pressure-induced phase transition in $C u I n S e_{2}$. Physical review B, 45(13):7022-7025, 1992.

[26] J. D. Grice and R. B. Ferguson. Crystal structure refinement of Millerite -NiS. Canadian Mineralogist, 12:248-252, 1974.

[27] Th. (ed.) Hahn. International Tables for Crystallography Vol. A. International Union of Crystallography, 1 edition, 2006.

[28] A. Hammersley. FIT2D: An Introduction and Overview. ESRF Internal Report, 97:HA02T, 1997.

[29] A. Hammersley, S. Svensson, M. Hanfland, A. Fitch, and D. Häusermann. TwoDimensional Detector Software: From Real Detector to Idealised Image or TwoTheta Scan. High Pressure Research, 14:235-248, 1996.

[30] D. M. Hatch, H. T. Stokes, J. Dong, J. Gunter, H. Wang, and J. P. Lewis. Bilayer sliding mechansim for the zinc-blende to rocksalt transition in SiC. Physical Review B, 71:184109, 2005.

[31] C. He, C. Gao, Y. Maa, B. Liu, M. Li, X. Huang, A. Hao, C. Yu, D. Zhang, H. Liu, 
and G. Zou. Electrical property and phase transition of CdSe under high pressure. Journal of Physics and Chemistry of Solids, 69:2227-2229, 2008.

[32] U. Hotje, C. Rose, and M. Binnewies. Lattice constants and molar volume in the system ZnS, ZnSe, CdS, CdSe. Solid State Sciences, 5:1259-1262, 2003.

[33] B. G. Hyde and M. O'Keeffe. On Mechanisms of the B1 $\leftrightarrow$ B2 Structural Transformation. Phase transitions, edited by L. E. Cross, Oxford, Pergamon Press, 1:345349, 1973.

[34] J. Imhof. Determination of the Orientation Distribution Function From One Polefigure. Textures and Microstructures, 5:73-86, 1982.

[35] A. Jayaraman, W. Klement Jr., and G. C. Kennedy. Melting and polymorphic transitions for some group II-VI compounds at high pressures. Physical Review, 130:277-283, 1963.

[36] W. Kleber. Das Druck-Abstands-Paradoxon. Crystal Research and Technology, 2(1):13-14, 1967.

[37] M. D. Knudson and Y. M. Gupta. Transformation mechanism for the pressureinduced phase transition in shocked CdS. Physical Review B, 59:11704-11715, 1999.

[38] G. Kullerud and R. A. Yund. The Ni-S system and related minerals. Journal of Petrology, 3:126-175, 1962.

[39] A. Le Bail, H. Duroy, and J. Fourquet. Ab Initio Structure Determination of $\mathrm{LiSbWO}_{6}$ by X-ray Powder Diffraction. Materials Research Bulletin, 23:447-452, 1988.

[40] Y. Li, C. Lin, G. Li, J. Xu, X. Li, and J. Liu. Structure determination of the high-pressure phase of CdSe. Journal of Applied Physics, 115:223507, 2014.

[41] Y. Li, X. Zhang, H. Li, X. Li, C. Lin, W. Xiao, and J. Liu. High pressure-induced phase transitions in $C d S$ up to 1 Mbar. Journal of Applied Physics, 113:083509, 2013. 
[42] S. Limpijumnong and W. R. L. Lambrecht. Homogeneous Strain Deformation Path for the Wurtzite to Rocksalt High-Pressure Phase Transition in GaN. Physical Review Letters, 86:91-94, 2001.

[43] L. Lutterotti, S. Matthies, and H. Wenk. MAUD (Material Analysis Using Diffraction): A user friendly Java program for Rietveld texture analysis and more. J.A. Szpunar (ed.): Proc. 12th Intern. Conf. Textures of Materials (ICOTOM-12), NRC Research Press, Ottawa (Canada), 2:1599-1604, 1999.

[44] H. Mao and P. Bell. Design and Operation of a Diamond-Window, High-Pressure Cell for the Study of Single-Crystal Samples Loaded Cryogenically. Carnegie Institution of Washington Yearbook, 79:409-411, 1980.

[45] S. Matthies and Vinel. G. On the Reproduction of the Orientation Distribution Function of Texturized Samples from Reduced Pole Figures Using the Conception of a Conditional Ghost Correction. Physica Status Solidi (B), 112(2):K111-K114, 1982.

[46] J. R. Mei and V. Lemos. Photoluminescence on CdSe and CdTe under hydrostatic pressure. Solid State Communications, 52:785-788, 1984.

[47] L. Merrill and A. Bassett. Miniature diamond anvil pressure cell for single crystal x-ray diffraction studies. Review of Scientific Instruments, 45(2):290-294, 1974.

[48] M. S. Miao and W. R. L. Lambrecht. Unified path for high-pressure transitions of SiC polytypes to the rocksalt structure. Physical Review B, 68:092103 1-4, 2003.

[49] S. Minomura, G. A. Samara, and H. G. Drickamer. Temperature coefficient of restistance of the high pressure phases of $\mathrm{Si}, \mathrm{Ge}$, and some III-V and II-VI compounds. Journal of Applied Physics, 33:3196-3197, 1962.

[50] C.A. Mullan, C. J. Kiely, S. M. Casey, M. Imanieh, M. V. Yakushev, and R. D. Tomlinson. A microstructural and compositional analysis of $\mathrm{CuInSe_{2 }}$ ingots grown by the vertical Bridgman technique. Journal of Crystal Growth, 171:415-424, 1997.

[51] A. Neuhaus. Synthese, Strukturverhalten und Valenzzustände der anorganischen Materie im Bereich hoher und höchster Drücke. Chimia, 18:93-103, 1964. 
[52] A. Onodera. Temperature coefficient of restistance of the high pressure phases of Si, Ge, and some III-V and II-VI compounds. The Review of Physical Chemistry of Japan, 39:65-77, 1970.

[53] N. B. Owen, P. L. Smith, J. E. Martin, and A. J. Wright. X-ray diffraction at ultra-high pressures. Journal of Physics and Chemistry of Solids, 24:1519-1520, 1963.

[54] G. J. Piermarini, S. Block, and J. D. Barnett. Hydrostatic limits in liquids and solids up to 100 kbar. Journal of Applied Physics, 44:5377, 1973.

[55] G. J. Piermarini, S. Block, and J. D. Barnett. Calibration of the pressure dependence of the R1 ruby fluorescence line to 195 kbar. Journal of Applied Physics, 46:2774, 1975 .

[56] L. Raue. Texturen und Eigenschaften biologischer Materialien. Mensch und Buch Verlag, Berlin, 1 edition, 2008.

[57] L. Raue. input4Maud: an efficient program for automatic two-dimensional diffraction series input and/or batch refinement with MAUD. Journal of Applied Crystallography, 47:2081-2085, 2014.

[58] H. M. Rietveld. A profile refinement for nuclear and magnetic structures. Journal of Applied Crystallography, 2:65-71, 1969.

[59] E. Rogacheva, T. Tavrina, and L. Gladkikh. Non-Stochiometry and defect Structure of CuInSe $e_{2}$. Japanese Journal of Applied Physics, 39:397-398, 2000.

[60] C. J. M. Rooymans. Structure of the high pressure phase of CdS, CdSe and InSb. Physics Letters, 4:186-187, 1963.

[61] C. J. M. Rooymans. A study of the structure of cadmium sulfide at high pressures up to 90 kilobars. Soviet Physics-Doklady, 8:800-802, 1964.

[62] G. A. Samara and A. A. Giardini. Compressibility and electrical conductivity of cadmium sulfide at high pressures. Physical Review, 140:A388-A359, 1965. 
[63] J. Schwinger. On the classical radiation of accelerated electrons. Physical Review, 75:1912-1925, 1949.

[64] S. M. Sharma and Y. M. Gupta. Wurtzite-to-rocksalt structural transformation in cadmium sulphide shocked along the a axis. Physical Review B, 58:5964-5971, 1998.

[65] F. Shimojo, I. Ebbsjo, R. K. Kalia, A. Kakano, J. P. Rino, and P. Vashishta. Molecular dynamics simulation of structural transformation in silicon carbide unter pressure. Physical Review Letters, 84:3338-3341, 2000.

[66] F. Shimojo, S. Kodiyalam, I. Ebbsjo, R. K. Kalia, A. Kakano, and P. Vashishta. Molecular dynamics simulation of structural transformation in silicon carbide unter pressure. Physical Review B, 70:184111, 2004.

[67] H. Shôji. Geometrische Beziehungen unter den Strukturen der Modifikationen einer Substanz. Zeitschrift für Kristallographie, 77:381-410, 1931.

[68] H. Sowa. A transition path from the zinc-blende to the $\mathrm{NaCl}$ type. Zeitschrift für Kristallographie, 215:335-342, 2000.

[69] H. Sowa. On the transition from the wurtzite to the $\mathrm{NaCl}$ type. Acta Crystallographica, A57:176-182, 2001.

[70] H. Sowa. Relations between the zinc-blende and the $\mathrm{NaCl}$ structure type. Acta Crystallographica, A59:266-272, 2003.

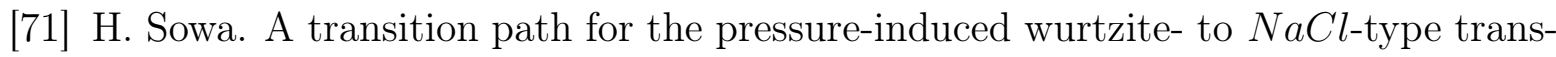
formation described in Pna2 $2_{1}$. Acta Crystallographica, A61:325-330, 2005.

[72] H. Sowa. On the mechanism of the pressure-induced wurtzite- to NaCl-type phase transition in CdS: an X-ray diffraction study. Solid State Sciences, 7:73-78, 2005.

[73] H. Sowa. The high-pressure behaviour of CdSe up to $3 \mathrm{GPa}$ and the orientation relations between its wurtzite- and NaCl-type modifications. Solid State Sciences, $7: 1384-1389,2005$. 
[74] H. Sowa and H. Ahsbahs. High-pressure X-ray investigation of Zincite $Z n O$ single crystals using diamond anvils with an improved shape. Journal of Applied Crystallography, 39:169-175, 2006.

[75] H. Sowa, H. Ahsbahs, and W. Schmitz. X-ray diffraction studies of millerite NiS under non-ambient conditions. Physics and Chemistry of Minerals, 31:321-327, 2009 .

[76] H. Sowa and H. Klein. Synchrotron investigation of the high-pressure phase transition in InAs at 7 GPa. HASYLAB annal report, 20:1-2, 2009.

[77] H. Sowa, H. Klein, and L. Raue. Determination of the orientation relations between the low- and high-temperature phases of NiS. Solid State Phenomena, 160:177-182, 2010 .

[78] A. W. Stevenson, M. Milanko, and Z. Barnea. Anharmonic thermal vibrations and the position parameter in wurtzite structures. I. Cadmium sulphide. Acta Crystallographica B, 40:521-530, 1984.

[79] A. W. Stevenson, M. Milanko, and Z. Barnea. Anharmonic thermal vibrations and the position parameter in wurtzite structures. II. Cadmium selenide. Acta Crystallographica B, 40:530-537, 1984.

[80] H. T. Stokes, J. Gunter, D. M. Hatch, J. Dong, H. Wang, and J. P. Lewis. Bilayer sliding mechanism for the wurtzite-to-rocksalt transition. Physical Review B, 76:012102, 2007.

[81] T. Tinoco, A. Polian, D. Gómez, and J. P. Itié. Structural Studies of CuIn $S_{2}$ and CuInSe $e_{2}$ under High Pressure. physica status solidi (b), 198:433-438, 1996.

[82] S. H. Tolbert and A. P. Alivisatos. The wurtzite to rock salt structural transformation in CdSe nanocrystals unter high pressure. Journal of Chemical Physics, 102:4642-4656, 1995.

[83] J. Trahan, R. G. Goodrich, and S. F. Watkins. X-ray diffraction measurements on metallic and semiconducting hexagonal NiS. Physical Review B, 2:2859-2862, 1970. 
[84] S. Ves, W. Hönle, and K. Syassen. Raman scattering and phase changes of CulnSe and $\mathrm{LinSe}_{2}$ at high pressure. High Pressure Research, 9:36-40, 1992.

[85] Y. Vohra, S. Weir, and A. Ruoff. High-pressure phase transitions and equation of state of the III-V compound InAs op to 27 GPa. Physical Review B, 31(11):7344$7348,1985$.

[86] G. Wassermann and J. Grewen. Texturen metallischer Werkstoffe. Springer Verlag, Berlin, 2 edition, 1962.

[87] L. Wcislak, H. Klein, H. J. Bunge, U. Garbe, T. Tschentscher, and J.R. Schneider. Texture analysis with high-energy synchrotron radiation. Journal of Applied Crystallography, 35:82, 2002.

[88] H. Wenk, S. Matthies, J. Donovan, and D. Chateigner. BEARTEX: a Windowsbased program system for quantitative texture analysis. Journal of Applied Crystallography, 31:262-269, 1998.

[89] Herhold B. A. Wickham, J. N. and A. P. Alivisatos. Shape Change as an Indicator of Mechanism in the High-Pressure Structural Transformations of CdSe Nanocrystals. Physical Review Letters, 84:923-926, 2000.

[90] R. Williams. Analytical Methods for Representing Complex Textures by Biaxial Pole Figures. Journal of Applied Physics, 39:4329-4335, 1968.

[91] G. Wulff. Untersuchungen im Gebiete der optischen Eigenschaften isomorpher Kristalle. Zeitschrift für Kristallographie, 36:1-28, 1902.

[92] M. Ye, R. Tang, S. Ma, Q. Tao, X. Wang, Y. Li, and P. Zhu. Electrical transport properties and band structure of $\mathrm{CuInSe} \mathrm{S}_{2}$ under high pressure. Journal of Physical Chemistry C, 123:20757-20763, 2019.

[93] W. C. Yu and P. J. Gielisse. High pressure polymorphism in CdS, CdSe and CdTe. Materials Research Bulletin, 6:621-638, 1971. 


\section{Anhang}
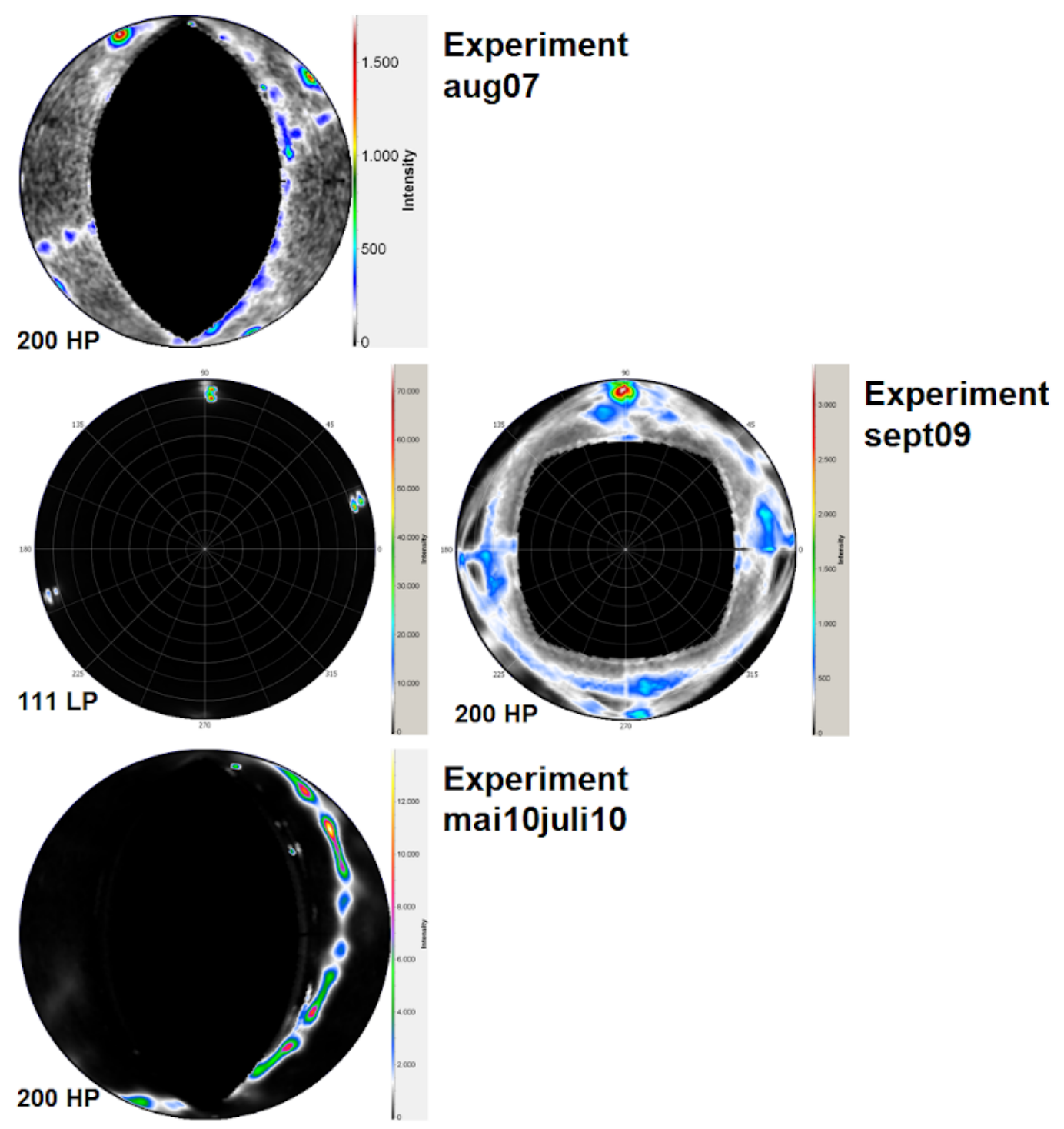

Abbildung 10.1: Alle gemessenen Polfiguren der InAs-Experimente aug07, sept09 und mai10juli10. 


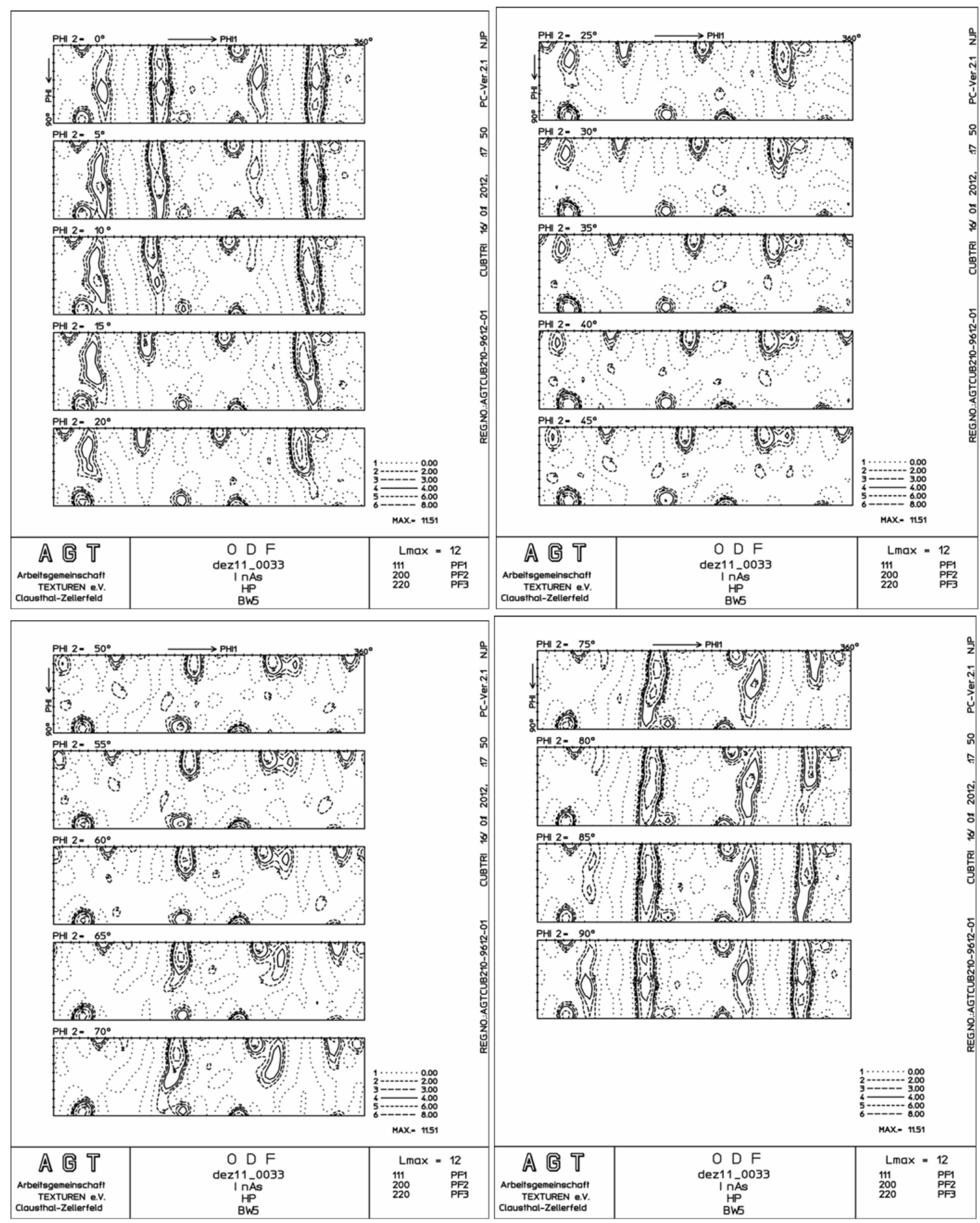

Abbildung 10.2: Vollständige Eulerraumschnitte $0^{\circ} \leq \varphi_{2} \leq 90^{\circ}$ der Hochdruckphase von InAs im $N a C l$-Typ für das Experiment dez11. Die Schrittweite beträgt $\Delta \varphi_{2}=5^{\circ}$. 


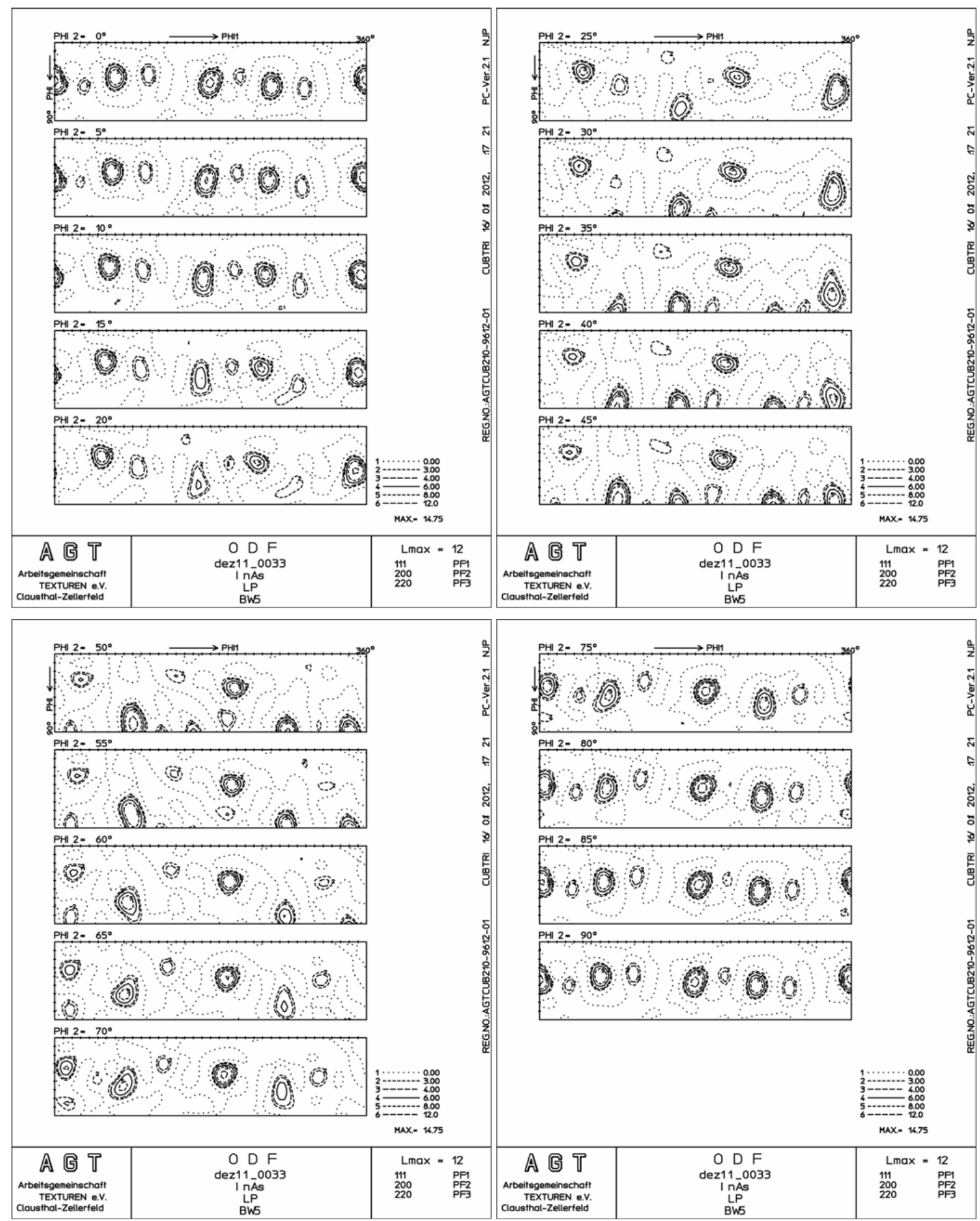

Abbildung 10.3: Vollständige Eulerraumschnitte $0^{\circ} \leq \varphi_{2} \leq 90^{\circ}$ der entlasteten polykristallinen Niederdruckphase im Zinkblende-Typ von InAs für das Experiment dez11. Die Schrittweite beträgt $\Delta \varphi_{2}=5^{\circ}$. 


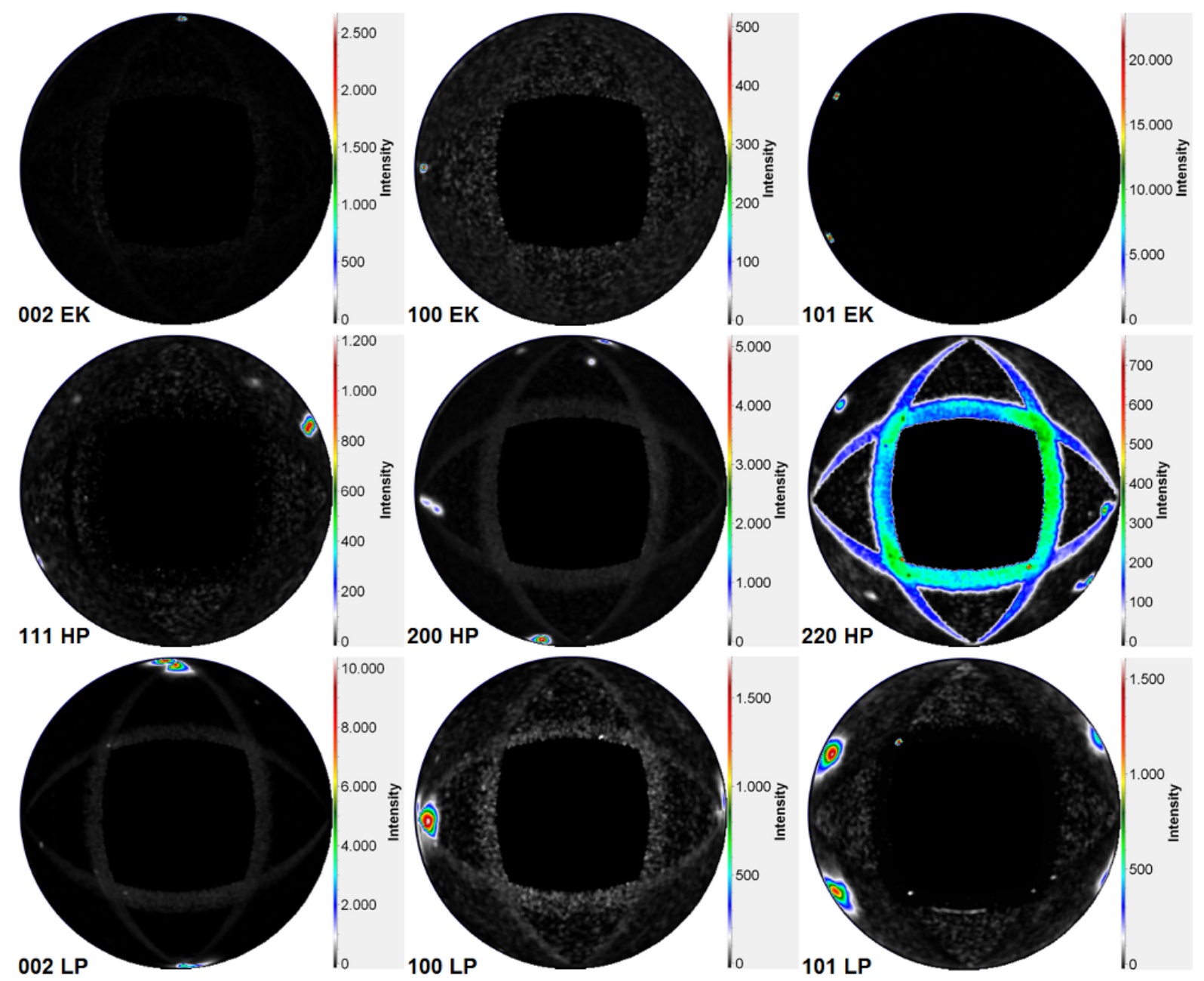

Abbildung 10.4: Alle gemessenen Polfiguren des $C d S$-Experiments juli09. 


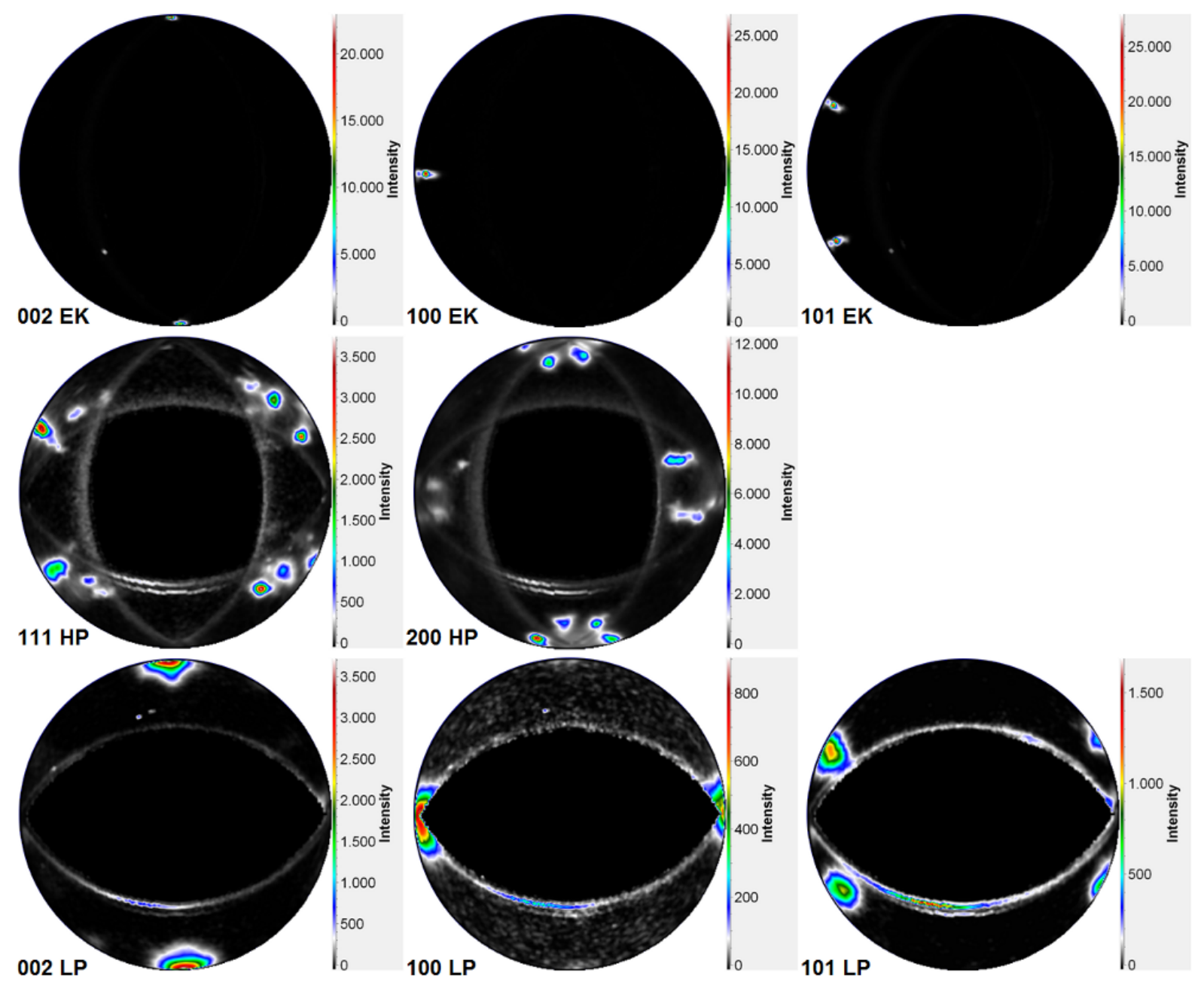

Abbildung 10.5: Alle gemessenen Polfiguren des $C d S$-Experiments sept10. 


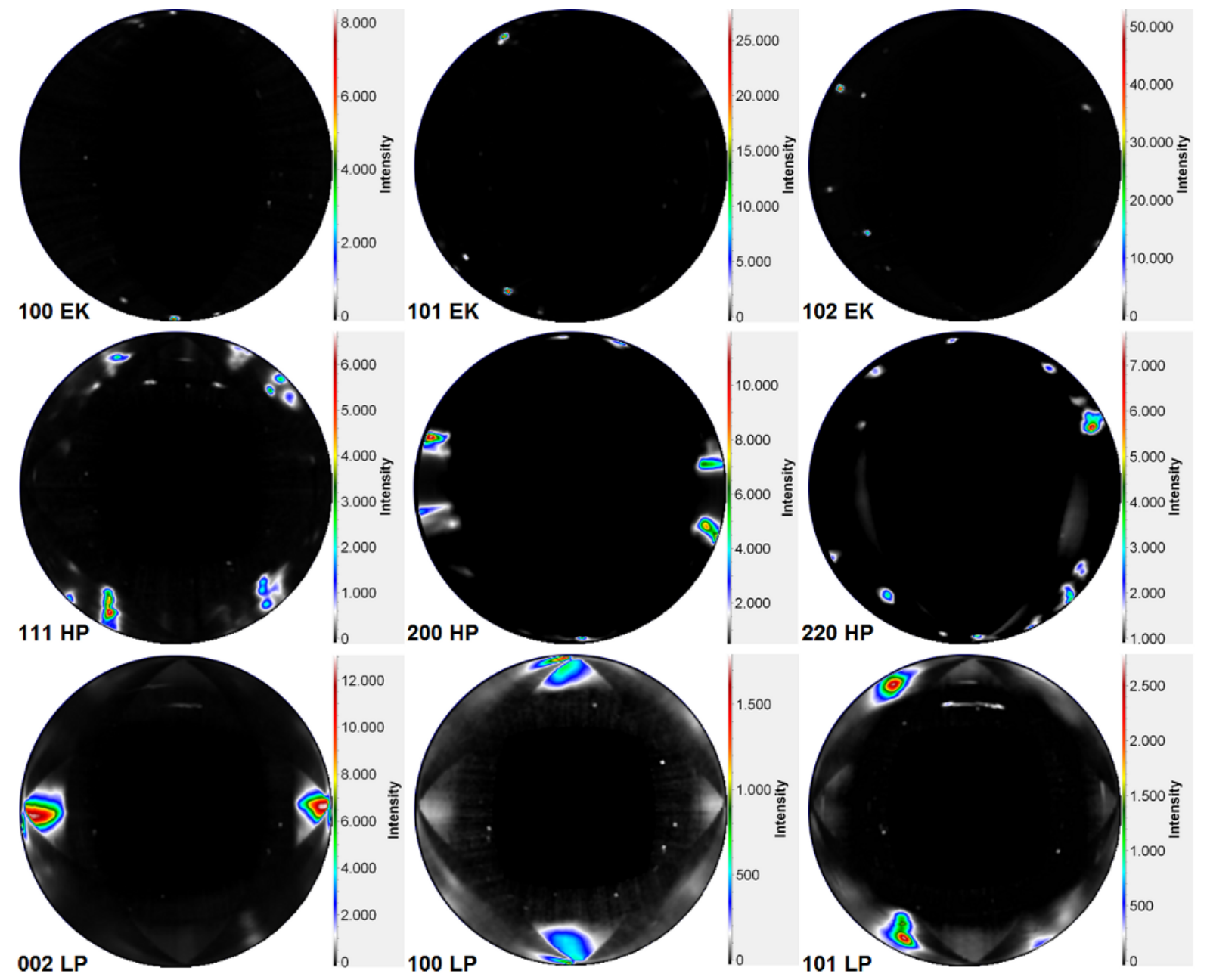

Abbildung 10.6: Alle gemessenen Polfiguren des $C d S$-Experiments juni13. 


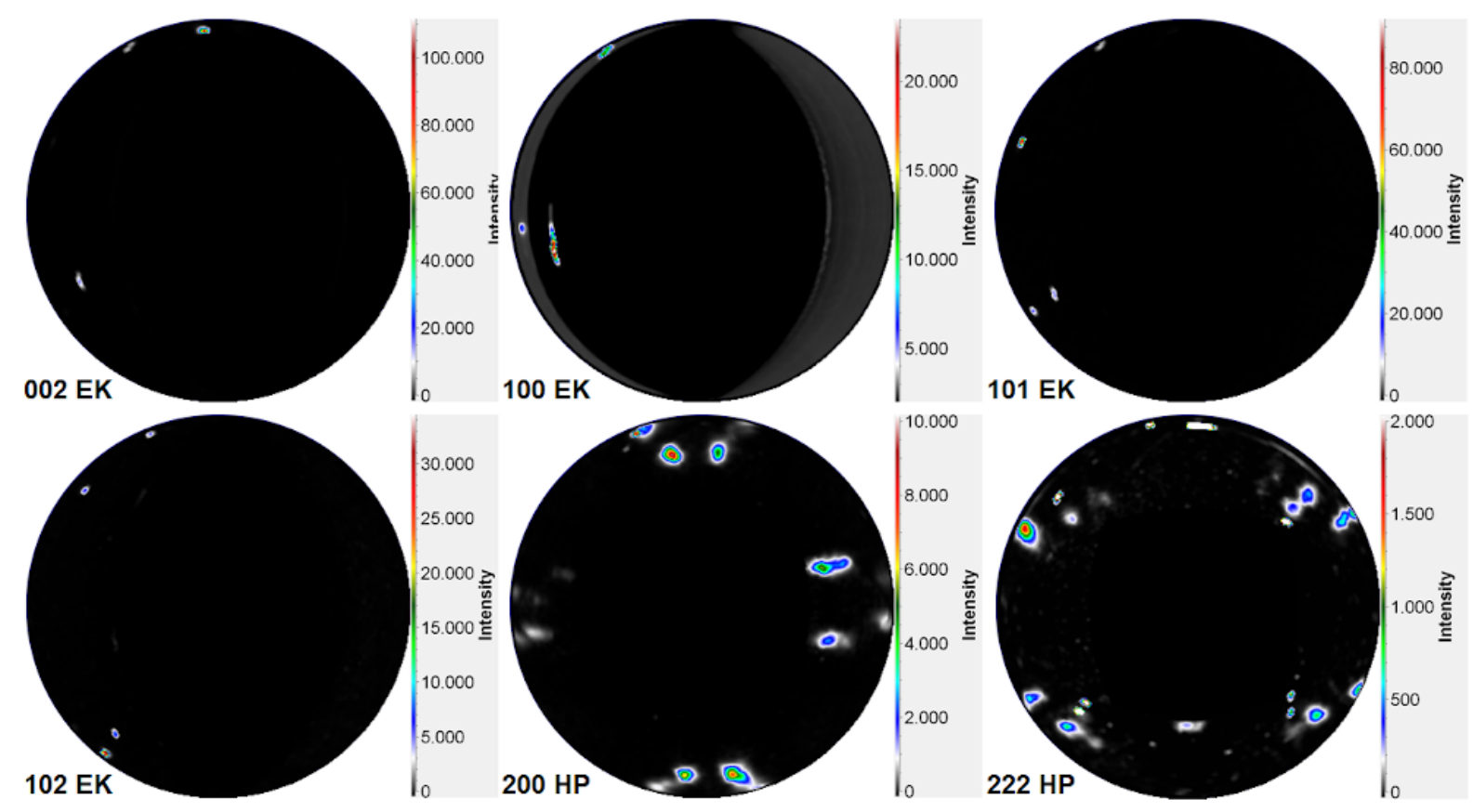

Abbildung 10.7: Alle gemessenen Polfiguren des CdSe-Experiments mai11. 


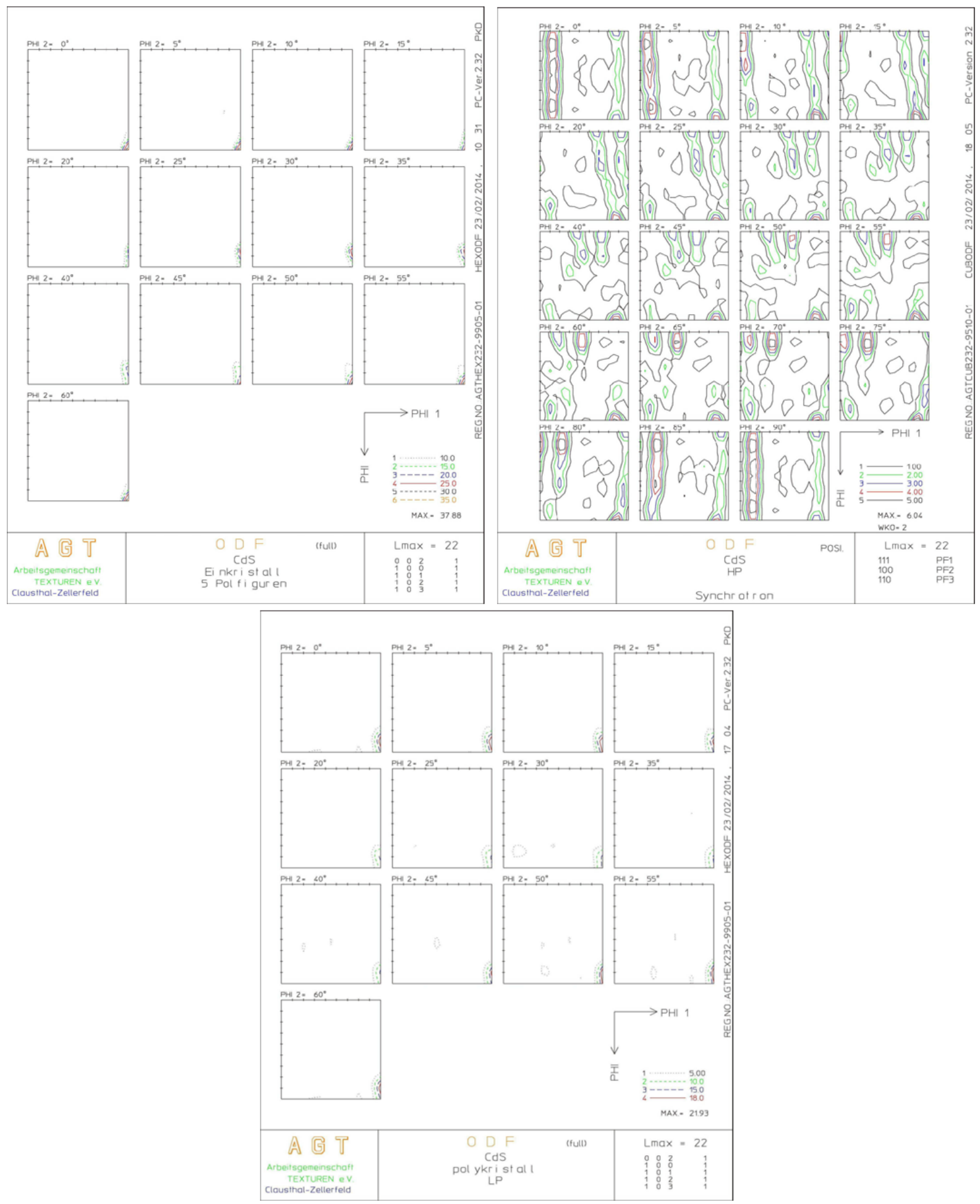

Abbildung 10.8: Vollständige Eulerraumschnitte des $C d S$-Experiments okt13. Für den Einkristall und die entlastete polykristalline Niederdruckphase im Wurtzit-Typ worden die Schnitte

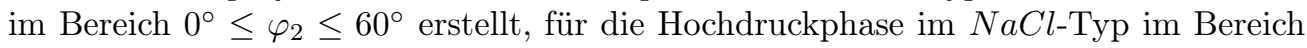
$0^{\circ} \leq \varphi_{2} \leq 90^{\circ}$. Die Schrittweite beträgt $\Delta \varphi_{2}=5^{\circ}$. 


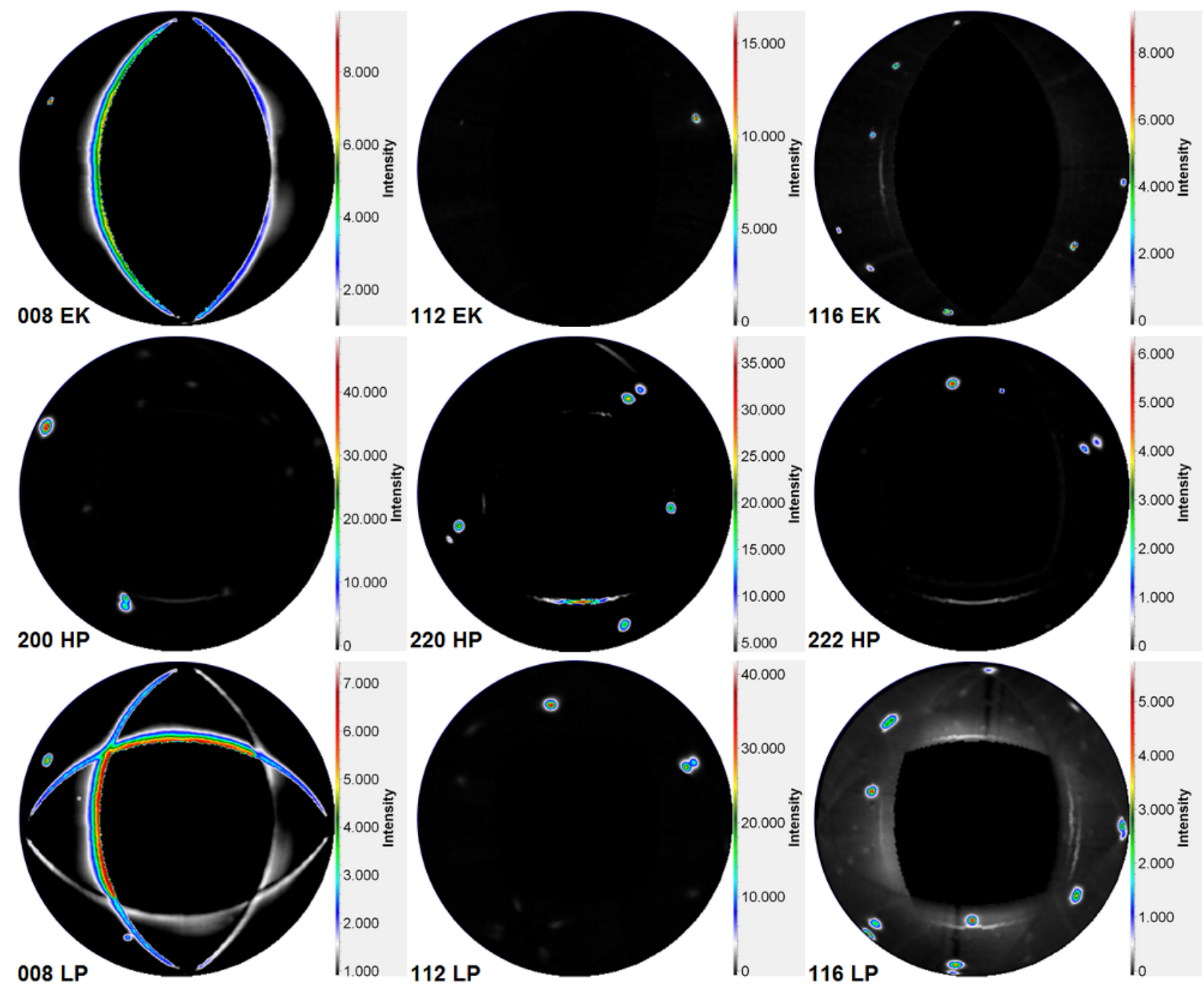

Abbildung 10.9: Alle gemessenen Polfiguren des $\mathrm{CuInSe}_{2}$-Experiments junjul12. 

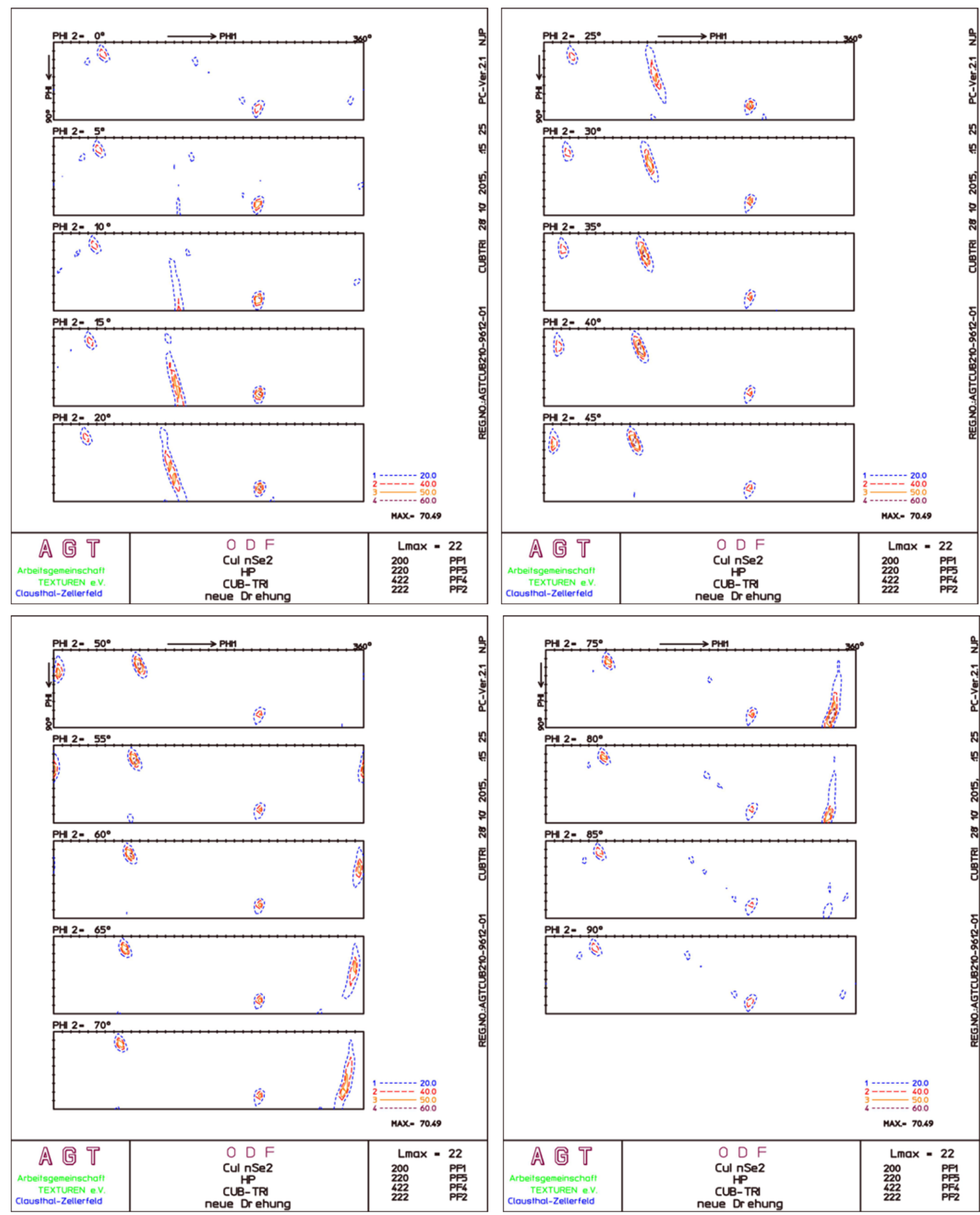

Abbildung 10.10: Vollständige Eulerraumschnitte $0^{\circ} \leq \varphi_{2} \leq 90^{\circ}$ der Hochdruckphase von $\mathrm{CuInSe_{2 }}$ im $\mathrm{NaCl}$-Typ für das Experiment junjul12. Die Schrittweite beträgt $\Delta \varphi_{2}=5^{\circ}$. 